[supporting information]

\title{
Asymmetric linear-selective hydroformylation of 1,1-dialkyl olefins assisted by a steric-auxiliary strategy
}

Dequan Zhang ${ }^{\dagger}$, Cai You ${ }^{\dagger}$, Xiuxiu $\mathrm{Li}^{\dagger}$, Jialin Wen*t, ${ }^{\dagger}$ and Xumu Zhang* ${ }^{\dagger}$

† Shenzhen Grubbs Institute and department of chemistry, Southern University of Science and Technology, 1088 Xueyuan Road, Shenzhen, 518055, China.

$\$$ Academy for Advanced Interdisciplinary Studies, Southern University of Science and Technology, 1088 Xueyuan Road, Shenzhen, 518055, China.

\section{Contents}

1. General Remarks S2

2. General Procedure for the Synthesis of substrates. S3

3. General Procedure for asymmetric hydroformylation

4. Characterization data for products.

5. Procedures for synthetic transformations S25

6. NMR spectra S28

7. HPLC chromatograms S75

References 


\section{General Remarks}

All the reactions dealing with air- or moisture- sensitive compounds were carried out in a dry reaction vessel under an argon atmosphere or in an argon-filled glove box. Unless otherwise noted, all reagents and solvents were purchased from commercial suppliers without further purification. Toluene was dried with sodium chips and indicated by benzophenone. Other anhydrous solvents were purchased from J\&K Chemical and degassed by bubbling argon over a period of $30 \mathrm{~min}$. Purification of products was carried out by flash chromatography using silica gel (200-300 mesh). Thin layer chromatography (TLC) was performed on EM reagents $0.25 \mathrm{~mm}$ silica 60 -F plates. The metal precursor $\left(\mathrm{Rh}(\mathrm{acac})(\mathrm{CO})_{2}\right)$ was purchased from Heraeus.

${ }^{1} \mathrm{H},{ }^{13} \mathrm{C},{ }^{19} \mathrm{~F}$ and ${ }^{31} \mathrm{P}$ NMR spectra were recorded on a Bruker Avance $400 \mathrm{MHz}$ or on a Bruker Avance 600 $\mathrm{MHz}$ spectrometer with tetramethylsilane as the internal standard. Chemical shifts are reported in parts per million (ppm, $\delta$ scale) downfield from TMS at $0.00 \mathrm{ppm}$ and referenced to the $\mathrm{CDCl}_{3}$ at $7.27 \mathrm{ppm}$ for $1 \mathrm{H} \mathrm{NMR}$ or $77.0 \mathrm{ppm}$ for ${ }^{13} \mathrm{C}$ NMR. Data are reported as: multiplicity $(\mathrm{s}=$ singlet, $\mathrm{d}=$ doublet, $\mathrm{t}=$ triplet, $\mathrm{q}=$ quartet, $\mathrm{m}=$ multiplet), coupling constant in hertz $(\mathrm{Hz})$ and signal area integration in natural numbers. ${ }^{13} \mathrm{C}$ NMR and ${ }^{31} \mathrm{P}$ NMR analyses were recorded with ${ }^{1} \mathrm{H}$ decoupling. Enantiomeric excess values were determined Agilent 1260 Series HPLC instrument. Optical rotations were measured using a $1 \mathrm{~mL}$ cell with a $1 \mathrm{dm}$ path length on a Rudolph Autopol I polarimeter at $589 \mathrm{~nm}$. All new products were further characterized by HRMS. A positive ion mass spectrum of sample was acquired on a Thermo LTQ-FT mass spectrometer with an electrospray ionization source. Sonication was carried out with KQ5200DE ultrasonicator manufactured by Kunshan Ultrasonic Instrument Co., Ltd. Crystal structure was measured with BRUKER APEX III diffractometer. 


\section{General Procedure for the Synthesis of substrates}

Method A: (for compound 1a-1h)

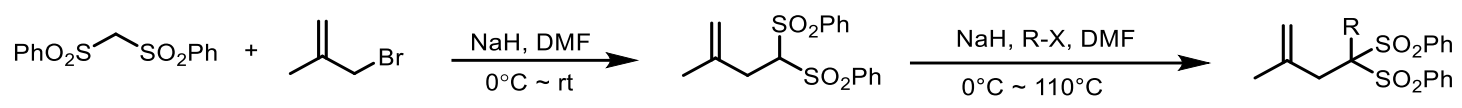

1a-1h was prepared according to the literature. ${ }^{1}$ Bis(benzenesulfonyl)methane (1 equiv.) and DMF (6 $\mathrm{ml} / \mathrm{mmol})$ were added to a 3 -neck round bottom flask. The reaction mixture was cooled to $0{ }^{\circ} \mathrm{C}$ and $\mathrm{NaH}(1.1$ equiv.) was added slowly. After hydrogen evolution had ceased, 3-bromo-2-methylprop-1-ene (1.5 equiv.) was added. The reaction mixture was warmed to room temperature and stirred until the reaction was complete (TLC monitoring). The reaction mixture was quenched with water and extracted with EtOAc (3 times). The combined organic layers were washed with water and brine, dried over anhydrous $\mathrm{MgSO}_{4}$ and concentrated under reduced pressure. Recrystallization in EtOH afforded the pure product.

(3-methylbut-3-ene-1,1-diyldisulfonyl) dibenzene (1 equiv.) and DMF (6 ml/mmol) were added to a 3 neck round bottom flask. The reaction mixture was cooled to $0{ }^{\circ} \mathrm{C}$ and $\mathrm{NaH}$ (1.1 equiv.) was added slowly. After hydrogen evolution had ceased, the electrophile (1.5 equiv.) was added and the reaction mixture was heated at $110^{\circ} \mathrm{C}$ (oil bath) and stirred overnight. The reaction mixture was quenched with water and extracted with EtOAc ( 3 times). The combined organic layers were washed with water and brine, dried over anhydrous $\mathrm{MgSO}_{4}$ and concentrated under reduced pressure. The resultant mixture was purified by flash column chromatography (on silica, petroleum ether/ ethyl acetate) to give the product.

Method B: (for compound 1i-1r)

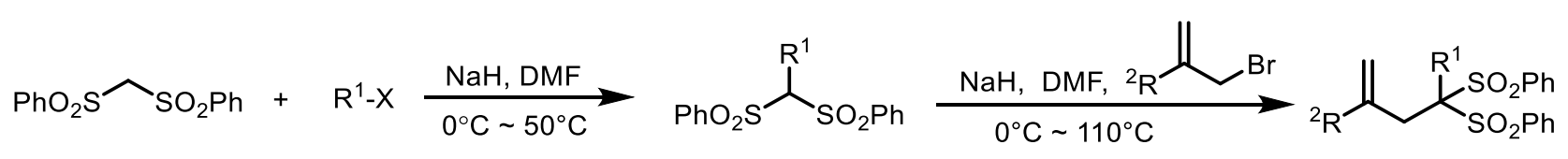

1i-1r was prepared according to the literature. ${ }^{1}$ Bis(benzenesulfonyl)methane (1 equiv.) and DMF (6 $\mathrm{ml} / \mathrm{mmol})$ were added to a 3 neck round bottom flask. The reaction mixture was cooled to $0{ }^{\circ} \mathrm{C}$ and $\mathrm{NaH}(1.1$ equiv.) was added slowly. After hydrogen evolution had ceased, the electrophile (1.5 equiv.) was added. The reaction mixture was heated at $50^{\circ} \mathrm{C}$ (oil bath) and stirred until the reaction was complete. The reaction mixture was quenched with water and extracted with EtOAc (3 times). The combined organic layers were washed with water and brine, dried over anhydrous $\mathrm{MgSO}_{4}$ and concentrated under reduced pressure. Concentration followed 
by recrystallization $(\mathrm{EtOH})$ afforded the pure product.

The solid sulfone (1.0 equiv.) and DMF (6 $\mathrm{ml} / \mathrm{mmol})$ were added to a 3 neck round bottom flask. The reaction mixture was cooled to $0{ }^{\circ} \mathrm{C}$ and $\mathrm{NaH}$ (1.1 equiv.) was added slowly. After hydrogen evolution had ceased, 3-bromo-2-methylprop-1-ene (1i-1p, 1.5 equiv.) or 2-(bromomethyl)but-1-ene (1q, 1.5 equiv.) or 2(bromomethyl)oct-1-ene (1r, 1.5 equiv.) was added and the reaction mixture was heated at $110{ }^{\circ} \mathrm{C}$ (oil bath) and stirred overnight. The reaction mixture was quenched with water and extracted with EtOAc ( 3 times). The combined organic layers were washed with water and brine, dried over anhydrous $\mathrm{MgSO}_{4}$ and concentrated under reduced pressure. The resultant mixture was purified by flash column chromatography (on silica, petroleum ether/ ethyl acetate) to give the product.

Method C: (for compound 1s, 1t)

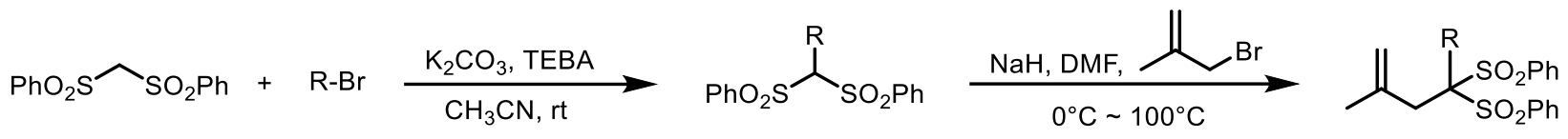

1s and 1t was prepared according to the literature. ${ }^{2}$ Bis(benzenesulfonyl)methane $(4.44 \mathrm{~g}, 15 \mathrm{mmol})$, Powdery $\mathrm{K}_{2} \mathrm{CO}_{3}(2.74 \mathrm{~g}, 20 \mathrm{mmol})$, TEBA $(0.3 \mathrm{~g})$ and $\mathrm{CH}_{3} \mathrm{CN}(20 \mathrm{ml})$ were added to a $100 \mathrm{ml}$ bottom flask and stirred for $15 \mathrm{~min}$ at at room temperature. Then the electrophile $(15 \mathrm{mmol})$ which was dissolved in $\mathrm{CH}_{3} \mathrm{CN}$ $(10 \mathrm{ml})$ was added slowly to the reaction mixture and stirred at room temperature until the reaction was complete. Water $(30 \mathrm{ml})$ was added and extracted with EtOAc $(20 \mathrm{~mL} \times 3)$. The combined organic layers were washed with brine, dried over anhydrous $\mathrm{MgSO}_{4}$ and concentrated under reduced pressure. Concentration followed by recrystallization in $\mathrm{EtOH}$ afforded the pure product.

The solid sulfone (1.0 equiv.) and DMF ( $6 \mathrm{ml} / \mathrm{mmol})$ were added to a 3 neck round bottom flask. The reaction mixture was cooled to $0{ }^{\circ} \mathrm{C}$ and $\mathrm{NaH}$ (1.1 equiv.) was added slowly. After hydrogen evolution had ceased, 3-bromo-2-methylprop-1-ene (1.5 equiv.) was added and the reaction mixture was heated at $100{ }^{\circ} \mathrm{C}$ (oil bath) and stirred overnight. The reaction mixture was quenched with water and extracted with EtOAc (3 times). The combined organic layers were washed with water and brine, dried over anhydrous $\mathrm{MgSO}_{4}$ and concentrated under reduced pressure. The resultant mixture was purified by flash column chromatography (on silica, petroleum ether/ ethyl acetate) to give the pure product. 
Method D: (for compound 1u)

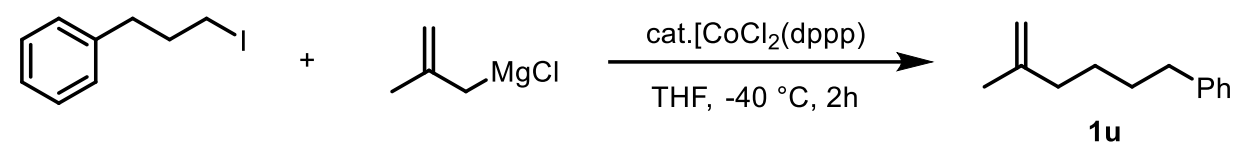

1u was prepared according to the literature. ${ }^{3}$ Anhydrous cobalt(ii) chloride ( $\left.65 \mathrm{mg}, 0.5 \mathrm{mmol}\right)$ was placed in a $100 \mathrm{~mL}$ flask and heated in vacuo with a hair dryer for $2 \mathrm{~min}$. After the color of the cobalt salt had changed to blue, dppp (250 mg, $0.6 \mathrm{mmol})$ and anhydrous THF $(10 \mathrm{~mL})$ were sequentially added under argon. The mixture was stirred for about $10 \mathrm{~min}$ at room temperature. (3-iodopropyl)benzene $(1.23 \mathrm{~g}, 5 \mathrm{mmol})$ and allylmagnesium chloride $(0.5 \mathrm{M}$ THF solution, $30 \mathrm{~mL}, 15 \mathrm{mmol})$ were successively added dropwise to the reaction mixture at $-40{ }^{\circ} \mathrm{C}$. While the Grignard reagent was being added, the mixture turned reddish-brown. After having been stirred at $-40{ }^{\circ} \mathrm{C}$ for $2 \mathrm{~h}$, the reaction mixture was poured into saturated ammonium chloride solution. The products were extracted with ethyl acetate EtOAc (3 times). The combined organic layers were dried over $\mathrm{Na}_{2} \mathrm{SO}_{4}$ and concentrated. Silica gel column purification (on silica, petroleum ether) of the crude product provided (5-methylhex-5-en-1-yl)benzene (1u) (601 mg, 69\% yield).<smiles>C=C(C)CC(C)(CC(C)(SO)S(=O)(=O)Oc1ccccc1)S(=O)(=O)c1ccccc1</smiles>

(4-methylpent-4-ene-2,2-diyldisulfonyl)dibenzene (1a): purified by flash chromatography (on silica, petroleum ether/ ethyl acetate $=4 / 1$, v/v), white solid, $3.47 \mathrm{~g}, 92 \%$ yield (for two steps); ${ }^{1} \mathrm{H}$ NMR (400 MHz, $\left.\mathrm{CDCl}_{3}\right) \delta: 8.02(\mathrm{~d}, J=8.5 \mathrm{~Hz}, 4 \mathrm{H}), 7.71(\mathrm{t}, J=7.4 \mathrm{~Hz}, 2 \mathrm{H}), 7.62-7.56(\mathrm{~m}, 4 \mathrm{H}), 4.99(\mathrm{~s}, 1 \mathrm{H}), 4.73(\mathrm{~s}, 1 \mathrm{H}), 2.95$ (s, 2H), $1.84-1.80(\mathrm{~m}, 6 \mathrm{H}) .{ }^{13} \mathrm{C}$ NMR $\left(101 \mathrm{MHz}, \mathrm{CDCl}_{3}\right) \delta: 138.3,136.4,134.5,131.4,128.7,118.9,87.7$, 37.6, 24.5, 16.9 ppm. HRMS (ESI-TOF) $\mathrm{m} / \mathrm{z}:[\mathrm{M}+\mathrm{Na}]^{+}$Calcd for $\mathrm{C}_{18} \mathrm{H}_{20} \mathrm{O}_{4} \mathrm{~S}_{2}=387.0701$; Found 387.0694 .<smiles>C=C(C)CC(CC)(OSc1ccccc1)S(=O)(=O)Oc1ccccc1</smiles>

(5-methylhex-5-ene-3,3-diyldisulfonyl)dibenzene (1b): purified by flash chromatography (on silica, petroleum ether/ ethyl acetate $=4 / 1$, v/v), white solid, $1.90 \mathrm{~g}, 26 \%$ yield (for two steps); ${ }^{1} \mathrm{H}$ NMR (400 MHz, $\left.\mathrm{CDCl}_{3}\right) \delta: 8.06(\mathrm{~d}, J=7.6 \mathrm{~Hz}, 4 \mathrm{H}), 7.69(\mathrm{t}, J=7.4 \mathrm{~Hz}, 2 \mathrm{H}), 7.56(\mathrm{t}, J=7.8 \mathrm{~Hz}, 4 \mathrm{H}), 5.02(\mathrm{~d}, J=8.1 \mathrm{~Hz}, 2 \mathrm{H})$, $3.03(\mathrm{~s}, 2 \mathrm{H}), 2.46(\mathrm{q}, J=7.4 \mathrm{~Hz}, 2 \mathrm{H}), 1.72(\mathrm{~s}, 3 \mathrm{H}), 1.29(\mathrm{t}, J=7.4 \mathrm{~Hz}, 3 \mathrm{H}) .{ }^{13} \mathrm{C}$ NMR $\left(101 \mathrm{MHz}, \mathrm{CDCl}_{3}\right) \delta$ : 138.0, 137.3, 134.4, 131.5, 128.5, 118.1, 93.4, 35.6, 24.7, 23.0, 9.4 ppm. HRMS (ESI-TOF) m/z : [M+Na] ${ }^{+}$ Calcd for $\mathrm{C}_{19} \mathrm{H}_{22} \mathrm{O}_{4} \mathrm{~S}_{2}=401.0857$; Found 401.0581 . 


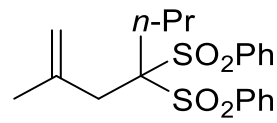

(2-methylhept-1-ene-4,4-diyldisulfonyl)dibenzene (1c): purified by flash chromatography (on silica, petroleum ether/ ethyl acetate $=5 / 1$, v/v), white solid, $2.70 \mathrm{~g}, 29 \%$ yield (for two steps); ${ }^{1} \mathrm{H} \mathrm{NMR}(400 \mathrm{MHz}$, $\left.\mathrm{CDCl}_{3}\right) \delta: 8.05(\mathrm{~d}, J=7.3 \mathrm{~Hz}, 4 \mathrm{H}), 7.69(\mathrm{t}, J=7.4 \mathrm{~Hz}, 2 \mathrm{H}), 7.57(\mathrm{t}, J=7.8 \mathrm{~Hz}, 4 \mathrm{H}), 5.02(\mathrm{~d}, J=4.8 \mathrm{~Hz}, 2 \mathrm{H})$, $3.02(\mathrm{~s}, 2 \mathrm{H}), 2.42-2.20(\mathrm{~m}, 2 \mathrm{H}), 1.87-1.66(\mathrm{~m}, 5 \mathrm{H}), 0.92(\mathrm{t}, J=7.3 \mathrm{~Hz}, 3 \mathrm{H}) .{ }^{13} \mathrm{C} \mathrm{NMR}\left(101 \mathrm{MHz}, \mathrm{CDCl}_{3}\right) \delta$ : 138.0, 137.2, 134.4, 131.5, 128.5, 118.0, 93.4, 35.9, 31.6, 24.7, 17.8, 14.5 ppm. HRMS (ESI-TOF) m/z : $[\mathrm{M}+\mathrm{Na}]^{+}$Calcd for $\mathrm{C}_{20} \mathrm{H}_{24} \mathrm{O}_{4} \mathrm{~S}_{2}=415.1014 ;$ Found 415.1008

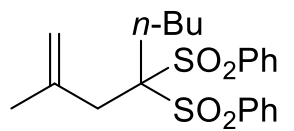

(2-methyloct-1-ene-4,4-diyldisulfonyl)dibenzene (1d): purified by flash chromatography (on silica, petroleum ether/ ethyl acetate $=6 / 1, \mathrm{v} / \mathrm{v})$, white solid, $2.72 \mathrm{~g}, 28 \%$ yield (for two steps) $;{ }^{1} \mathrm{H} \mathrm{NMR}(400 \mathrm{MHz}$, $\left.\mathrm{CDCl}_{3}\right) \delta: 8.06(\mathrm{~d}, J=7.3 \mathrm{~Hz}, 4 \mathrm{H}), 7.69(\mathrm{t}, J=7.4 \mathrm{~Hz}, 2 \mathrm{H}), 7.57(\mathrm{t}, J=7.8 \mathrm{~Hz}, 4 \mathrm{H}), 5.02(\mathrm{~d}, J=7.8 \mathrm{~Hz}, 2 \mathrm{H})$, $3.02(\mathrm{~s}, 2 \mathrm{H}), 2.44-2.24(\mathrm{~m}, 2 \mathrm{H}), 1.79-1.65(\mathrm{~m}, 5 \mathrm{H}), 1.36-1.25(\mathrm{~m}, 2 \mathrm{H}), 0.91(\mathrm{t}, J=7.4 \mathrm{~Hz}, 3 \mathrm{H}) .{ }^{13} \mathrm{C} \mathrm{NMR}$ $\left(101 \mathrm{MHz}, \mathrm{CDCl}_{3}\right) \delta: 138.0,137.3,134.4,131.5,128.4,117.9,93.4,35.8,29.4,26.2,24.7,23.3,13.7 \mathrm{ppm}$. HRMS (ESI-TOF) m/z : [M+Na $]^{+}$Calcd for $\mathrm{C}_{21} \mathrm{H}_{26} \mathrm{O}_{4} \mathrm{~S}_{2}=429.1170$; Found 429.1167 .

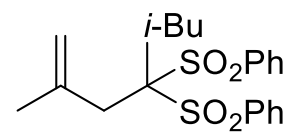

(2,6-dimethylhept-1-ene-4,4-diyldisulfonyl)dibenzene (1e): purified by flash chromatography (on silica, petroleum ether/ ethyl acetate $=6 / 1, \mathrm{v} / \mathrm{v}$ ), white solid, $420 \mathrm{mg}, 6 \%$ yield (for two steps); ${ }^{1} \mathrm{H}$ NMR (400 MHz, $\left.\mathrm{CDCl}_{3}\right) \delta: 8.07(\mathrm{~d}, J=8.0 \mathrm{~Hz}, 4 \mathrm{H}), 7.69(\mathrm{t}, J=7.5 \mathrm{~Hz}, 2 \mathrm{H}), 7.56(\mathrm{t}, J=7.7 \mathrm{~Hz}, 4 \mathrm{H}), 5.11(\mathrm{~s}, 1 \mathrm{H}), 5.05(\mathrm{~s}, 1 \mathrm{H})$, $3.07(\mathrm{~s}, 2 \mathrm{H}), 2.47-2.36(\mathrm{~m}, 1 \mathrm{H}), 2.31(\mathrm{~d}, J=5.4 \mathrm{~Hz}, 2 \mathrm{H}), 1.67(\mathrm{~s}, 3 \mathrm{H}), 1.03(\mathrm{~d}, J=6.6 \mathrm{~Hz}, 6 \mathrm{H}) .{ }^{13} \mathrm{C} \mathrm{NMR}(101$ $\left.\mathrm{MHz}, \mathrm{CDCl}_{3}\right) \delta: 138.1,137.6,134.4,131.6,128.4,118.6,94.7,37.8,37.0,25.4,25.2,24.3$ ppm. HRMS (ESITOF) $\mathrm{m} / \mathrm{z}:[\mathrm{M}+\mathrm{Na}]^{+}$Calcd for $\mathrm{C}_{21} \mathrm{H}_{26} \mathrm{O}_{4} \mathrm{~S}_{2}=429.1170$; Found 429.1165 .

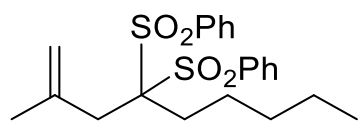

(2-methylnon-1-ene-4,4-diyldisulfonyl)dibenzene (1f): purified by flash chromatography (on silica, petroleum ether/ ethyl acetate $=10 / 1, \mathrm{v} / \mathrm{v})$, white solid, $1.57 \mathrm{~g}, 19 \%$ yield (for two steps); ${ }^{1} \mathrm{H}$ NMR $(400 \mathrm{MHz}$, $\left.\mathrm{CDCl}_{3}\right) \delta: 8.05(\mathrm{~d}, J=7.7 \mathrm{~Hz}, 4 \mathrm{H}), 7.68(\mathrm{t}, J=7.4 \mathrm{~Hz}, 2 \mathrm{H}), 7.56(\mathrm{t}, J=7.8 \mathrm{~Hz}, 4 \mathrm{H}), 5.02(\mathrm{~d}, J=6.0 \mathrm{~Hz}, 2 \mathrm{H})$, 
$3.02(\mathrm{~s}, 2 \mathrm{H}), 2.45-2.20(\mathrm{~m}, 2 \mathrm{H}), 1.72(\mathrm{~s}, 5 \mathrm{H}), 1.35-1.20(\mathrm{~m}, 4 \mathrm{H}), 0.88(\mathrm{t}, J=7.0 \mathrm{~Hz}, 3 \mathrm{H}) .{ }^{13} \mathrm{C}$ NMR $(101$ $\left.\mathrm{MHz}, \mathrm{CDCl}_{3}\right) \delta: 138.1,137.4,134.4,131.5,128.5,118.0,93.5,35.9,32.4,29.8,24.7,23.9,22.3,14.0 \mathrm{ppm}$. HRMS (ESI-TOF) m/z : [M+Na $]^{+}$Calcd for $\mathrm{C}_{22} \mathrm{H}_{28} \mathrm{O}_{4} \mathrm{~S}_{2}=443.1327$; Found 443.1321 .<smiles>C=C(C)CC(F)(OS(=O)(=O)c1ccccc1)S(=O)(=O)c1ccccc1</smiles>

(1-fluoro-3-methylbut-3-ene-1,1-diyldisulfonyl)dibenzene (1g): purified by flash chromatography (on silica, petroleum ether/ ethyl acetate $=4 / 1$, v/v), yellow oil, $1.20 \mathrm{~g}, 40 \%$ yield (for two steps); ${ }^{1} \mathrm{H}$ NMR $(400 \mathrm{MHz}$, $\left.\mathrm{CDCl}_{3}\right) \delta: 7.95-7.90(\mathrm{~m}, 4 \mathrm{H}), 7.74-7.68(\mathrm{~m}, 2 \mathrm{H}), 7.58-7.52(\mathrm{~m}, 4 \mathrm{H}), 4.90(\mathrm{~s}, 1 \mathrm{H}), 4.82(\mathrm{~s}, 1 \mathrm{H}), 3.21(\mathrm{~s}, 1 \mathrm{H})$, $3.15(\mathrm{~s}, 1 \mathrm{H}), 1.57(\mathrm{~s}, 3 \mathrm{H}) .{ }^{13} \mathrm{C} \mathrm{NMR}\left(101 \mathrm{MHz}, \mathrm{CDCl}_{3}\right) \delta 135.7,135.4,135.2,131.0,129.0,119.3,115.9(\mathrm{~d}, J$ $=271.1 \mathrm{~Hz}), 37.1(\mathrm{~d}, J=17.2 \mathrm{~Hz}), 23.0(\mathrm{~d}, J=3.1 \mathrm{~Hz})$. HRMS $\left(\right.$ ESI-TOF) $\mathrm{m} / \mathrm{z}:[\mathrm{M}+\mathrm{Na}]^{+}$Calcd for $\mathrm{C}_{17} \mathrm{H}_{17} \mathrm{FO}_{4} \mathrm{~S}_{2}=391.0450 ;$ Found 391.0444 .<smiles>C=C(C)CC(CC=C(C)C)(Sc1ccccc1)S(=O)(=O)c1ccccc1</smiles>

(2,7-dimethylocta-1,6-diene-4,4-diyldisulfonyl)dibenzene (1h): purified by flash chromatography (on silica, petroleum ether/ ethyl acetate $=8 / 1$, v/v), white solid, $3.21 \mathrm{~g}, 68 \%$ yield (for two steps); ${ }^{1} \mathrm{H} \mathrm{NMR}(400 \mathrm{MHz}$, $\left.\mathrm{CDCl}_{3}\right)$ 8: $7.96-7.92(\mathrm{~m}, 4 \mathrm{H}), 7.62-7.57(\mathrm{~m}, 2 \mathrm{H}), 7.49-7.43(\mathrm{~m}, 4 \mathrm{H}), 5.17(\mathrm{~s}, 1 \mathrm{H}), 4.95(\mathrm{~s}, 1 \mathrm{H}), 4.89(\mathrm{~s}, 1 \mathrm{H})$, $2.99(\mathrm{~d}, J=7.2 \mathrm{~Hz}, 4 \mathrm{H}), 1.74(\mathrm{~s}, 3 \mathrm{H}), 1.59$ (s, 3H). ${ }^{13} \mathrm{C} \mathrm{NMR}\left(101 \mathrm{MHz}, \mathrm{CDCl}_{3}\right) \delta: 138.2,137.5,135.9,134.4$, 131.6, 128.4 118.9, 116.2, 92.2, 36.4, 29.2, 26.0, 24.5, 18.2 ppm. HRMS (ESI-TOF) m/z : [M+Na $]^{+}$Calcd for $\mathrm{C}_{22} \mathrm{H}_{26} \mathrm{O}_{4} \mathrm{~S}_{2}=441.1170 ;$ Found 441.1165 .

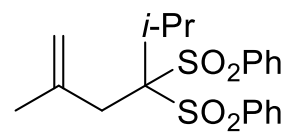

(2,5-dimethylhex-5-ene-3,3-diyldisulfonyl)dibenzene (1i): purified by flash chromatography (on silica, petroleum ether/ ethyl acetate $=5 / 1, \mathrm{v} / \mathrm{v})$, white solid, $1.52 \mathrm{~g}, 16 \%$ yield (for two steps); ${ }^{1} \mathrm{H}$ NMR $(400 \mathrm{MHz}$, $\left.\mathrm{CDCl}_{3}\right) \delta: 8.18(\mathrm{~d}, J=7.4 \mathrm{~Hz}, 4 \mathrm{H}), 7.68(\mathrm{t}, J=7.4 \mathrm{~Hz}, 2 \mathrm{H}), 7.55(\mathrm{t}, J=7.8 \mathrm{~Hz}, 4 \mathrm{H}), 5.24(\mathrm{~s}, 1 \mathrm{H}), 5.07(\mathrm{~s}, 1 \mathrm{H})$, $3.19(\mathrm{~h}, J=7.0 \mathrm{~Hz}, 1 \mathrm{H}), 3.00$ (s, 2H), 1.72 (s, 3H), 1.25 (d, $J=7.1 \mathrm{~Hz}, 6 \mathrm{H}) .{ }^{13} \mathrm{C} \mathrm{NMR}\left(101 \mathrm{MHz}, \mathrm{CDCl}_{3}\right) \delta$ : 139.6, 137.7, 134.3, 131.7, 128.4, 117.1, 99.1, 37.2, 29.8, 25.9, 19.4 ppm. HRMS (ESI-TOF) m/z : [M+Na $]^{+}$ Calcd for $\mathrm{C}_{20} \mathrm{H}_{24} \mathrm{O}_{4} \mathrm{~S}_{2}=415.1014$; Found 415.1009. 


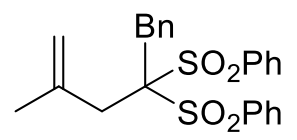

(4-methyl-1-phenylpent-4-ene-2,2-diyldisulfonyl)dibenzene (1j): purified by flash chromatography (on silica, petroleum ether/ ethyl acetate $=10 / 1$, v/v), white solid, $900 \mathrm{mg}, 18 \%$ yield (for two steps) ${ }^{1} \mathrm{H} \mathrm{NMR}(400 \mathrm{MHz}$, $\left.\mathrm{CDCl}_{3}\right) \delta: 7.76(\mathrm{~d}, J=7.7 \mathrm{~Hz}, 4 \mathrm{H}), 7.59(\mathrm{t}, J=7.4 \mathrm{~Hz}, 2 \mathrm{H}), 7.41(\mathrm{t}, J=7.8 \mathrm{~Hz}, 4 \mathrm{H}), 7.36-7.30(\mathrm{~m}, 2 \mathrm{H}), 7.28$ $-7.16(\mathrm{~m}, 3 \mathrm{H}), 5.25(\mathrm{~s}, 1 \mathrm{H}), 5.15(\mathrm{~s}, 1 \mathrm{H}), 3.88(\mathrm{~s}, 2 \mathrm{H}), 3.07$ (s, 2H), $1.90(\mathrm{~s}, 3 \mathrm{H}) .{ }^{13} \mathrm{C} \mathrm{NMR}\left(101 \mathrm{MHz}, \mathrm{CDCl}_{3}\right)$ $\delta: 138.1,137.9,134.1,133.4,131.7,131.4,128.4,128.4,127.6,119.1,94.0,37.7,35.9,25.5$ ppm. HRMS (ESITOF) $\mathrm{m} / \mathrm{z}:[\mathrm{M}+\mathrm{Na}]^{+}$Calcd for $\mathrm{C}_{24} \mathrm{H}_{24} \mathrm{O}_{4} \mathrm{~S}_{2}=463.1014$; Found 463.1009 .<smiles>C=C(C)CC(Cc1ccccc1)(Oc1ccccc1)S(=O)(=O)c1ccccc1</smiles>

(5-methyl-1-phenylhex-5-ene-3,3-diyldisulfonyl)dibenzene (1k): purified by flash chromatography (on silica, petroleum ether/ ethyl acetate $=10 / 1, \mathrm{v} / \mathrm{v})$, white solid, $2.90 \mathrm{~g}, 72 \%$ yield (for two steps); ${ }^{1} \mathrm{H}$ NMR $(400 \mathrm{MHz}$, $\left.\mathrm{CDCl}_{3}\right) \delta: 8.12-8.06(\mathrm{~m}, 4 \mathrm{H}), 7.73-7.67(\mathrm{~m}, 2 \mathrm{H}), 7.61-7.53(\mathrm{~m}, 4 \mathrm{H}), 7.31-7.26(\mathrm{~m}, 2 \mathrm{H}), 7.24-7.19(\mathrm{~m}$, 1H), $7.17-7.12(\mathrm{~m}, 2 \mathrm{H}), 5.11(\mathrm{~s}, 1 \mathrm{H}), 5.07(\mathrm{~s}, 1 \mathrm{H}), 3.12-3.05(\mathrm{~m}, 4 \mathrm{H}), 2.71-2.64(\mathrm{~m}, 2 \mathrm{H}), 1.78(\mathrm{~s}, 3 \mathrm{H}) .{ }^{13} \mathrm{C}$ NMR (101 MHz, $\left.\mathrm{CDCl}_{3}\right) \delta: 140.6,138.0,137.2,134.6,131.6,128.7,128.6,128.2,126.5,118.2,93.0,36.2$, 31.4, 30.4, 25.0 ppm. HRMS (ESI-TOF) m/z : $[\mathrm{M}+\mathrm{Na}]^{+}$Calcd for $\mathrm{C}_{25} \mathrm{H}_{26} \mathrm{O}_{4} \mathrm{~S}_{2}=477.1170$; Found 477.1166.

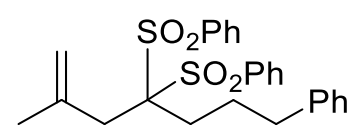

(2-methyl-7-phenylhept-1-ene-4,4-diyldisulfonyl)dibenzene (11): purified by flash chromatography (on silica, petroleum ether/ ethyl acetate $=15 / 1$, v/v), white solid, $718 \mathrm{mg}, 24 \%$ yield (for two steps); ${ }^{1} \mathrm{H}$ NMR (400 MHz, $\left.\mathrm{CDCl}_{3}\right) \delta: 8.04-7.87(\mathrm{~m}, 4 \mathrm{H}), 7.65(\mathrm{t}, J=7.4 \mathrm{~Hz}, 2 \mathrm{H}), 7.50(\mathrm{t}, J=7.8 \mathrm{~Hz}, 4 \mathrm{H}), 7.30-7.23(\mathrm{~m}, 2 \mathrm{H}), 7.22-$ $7.17(\mathrm{~m}, 1 \mathrm{H}), 7.15-7.06(\mathrm{~m}, 2 \mathrm{H}), 4.93(\mathrm{~d}, J=8.8 \mathrm{~Hz}, 2 \mathrm{H}), 2.98(\mathrm{~s}, 2 \mathrm{H}), 2.62(\mathrm{t}, J=7.1 \mathrm{~Hz}, 2 \mathrm{H}), 2.42-2.27$ (m, 2H), $2.11(\mathrm{~m}, 2 \mathrm{H}), 1.59$ (s, 3H). ${ }^{13} \mathrm{C}$ NMR (101 MHz, $\left.\mathrm{CDCl}_{3}\right) \delta: 140.8,137.9,137.2,134.4,131.5,128.7$, 128.5, 126.2, 118.0, 93.1, 36.1, 36.0, 29.3, 26.0, 24.7 ppm. HRMS (ESI-TOF) m/z : [M+Na $]^{+}$Calcd for $\mathrm{C}_{26} \mathrm{H}_{28} \mathrm{O}_{4} \mathrm{~S}_{2}=491.1327$; Found 491.1318 .

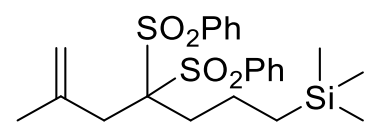

trimethyl(6-methyl-4,4-bis(phenylsulfonyl)hept-6-en-1-yl)silane (1m): purified by flash chromatography (on silica, petroleum ether/ ethyl acetate $=10 / 1, \mathrm{v} / \mathrm{v}$ ), white solid, $2.10 \mathrm{~g}, 74 \%$ yield (for two steps); ${ }^{1} \mathrm{H}$ NMR 
$\left(400 \mathrm{MHz}, \mathrm{CDCl}_{3}\right) \delta: 8.07(\mathrm{~d}, J=7.3 \mathrm{~Hz}, 4 \mathrm{H}), 7.71(\mathrm{t}, J=7.4 \mathrm{~Hz}, 2 \mathrm{H}), 7.58(\mathrm{t}, J=7.8 \mathrm{~Hz}, 4 \mathrm{H}), 5.04(\mathrm{~s}, 2 \mathrm{H})$, $3.06(\mathrm{~s}, 2 \mathrm{H}), 2.42-2.32(\mathrm{~m}, 2 \mathrm{H}), 1.76(\mathrm{~s}, 3 \mathrm{H}), 1.75-1.67(\mathrm{~m}, 2 \mathrm{H}), 0.50-0.41(\mathrm{~m}, 2 \mathrm{H}), 0.00(\mathrm{~s}, 9 \mathrm{H}) .{ }^{13} \mathrm{C} \mathrm{NMR}$ $\left(101 \mathrm{MHz}, \mathrm{CDCl}_{3}\right) \delta: 139.9,139.1,136.1,133.2,130.2,119.9,95.2,37.8,35.4,26.3,20.8,19.5,0.0$ ppm. HRMS (ESI-TOF) m/z : [M+Na $]^{+}$Calcd for $\mathrm{C}_{23} \mathrm{H}_{32} \mathrm{O}_{4} \mathrm{~S}_{2} \mathrm{Si}=487.1409$; Found 487.1403.

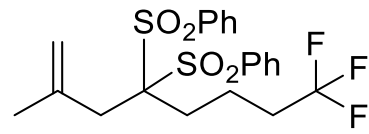

(8,8,8-trifluoro-2-methyloct-1-ene-4,4-diyldisulfonyl)dibenzene (1n): purified by flash chromatography (on silica, petroleum ether/ ethyl acetate $=10 / 1, \mathrm{v} / \mathrm{v})$, white solid, $2.75 \mathrm{~g}, 66 \%$ yield (for two steps); ${ }^{1} \mathrm{H}$ NMR $(400$ $\left.\mathrm{MHz}, \mathrm{CDCl}_{3}\right) \delta: 8.05(\mathrm{~d}, J=7.9 \mathrm{~Hz}, 4 \mathrm{H}), 7.71(\mathrm{t}, J=7.5 \mathrm{~Hz}, 2 \mathrm{H}), 7.58(\mathrm{t}, J=7.8 \mathrm{~Hz}, 4 \mathrm{H}), 5.00(\mathrm{~d}, J=16.7 \mathrm{~Hz}$, 2H), $3.01(\mathrm{~s}, 2 \mathrm{H}), 2.44-2.38(\mathrm{~m}, 2 \mathrm{H}), 2.14-1.97(\mathrm{~m}, 4 \mathrm{H}), 1.72(\mathrm{~s}, 3 \mathrm{H}) .{ }^{13} \mathrm{C} \mathrm{NMR}\left(151 \mathrm{MHz}, \mathrm{CDCl}_{3}\right) \delta 137.9$, 137.0, 134.7, 131.5, 128.7, $126.6(\mathrm{q}, J=276.8 \mathrm{~Hz}), 118.3,92.5,36.3,34.0(\mathrm{q}, J=28.5 \mathrm{~Hz}), 29.0,24.8,17.4(\mathrm{q}$, $J=3.3 \mathrm{~Hz}$ ). HRMS (ESI-TOF) m/z : [M+Na] $]^{+}$Calcd for $\mathrm{C}_{21} \mathrm{H}_{23} \mathrm{~F}_{3} \mathrm{O}_{4} \mathrm{~S}_{2}=483.0888$; Found 483.0881 .

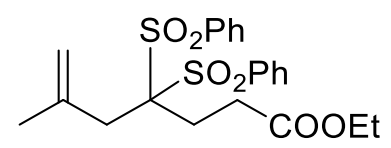

ethyl 6-methyl-4,4-bis(phenylsulfonyl)hept-6-enoate (10): purified by flash chromatography (on silica, petroleum ether/ ethyl acetate = 10/1, v/v), white solid, $2.30 \mathrm{~g}, 54 \%$ yield (for two steps); ${ }^{1} \mathrm{H} \mathrm{NMR}(400 \mathrm{MHz}$, $\left.\mathrm{CDCl}_{3}\right) \delta: 8.13-7.99(\mathrm{~m}, 4 \mathrm{H}), 7.76-7.67(\mathrm{~m}, 2 \mathrm{H}), 7.63-7.55(\mathrm{~m}, 4 \mathrm{H}), 5.00(\mathrm{~s}, 1 \mathrm{H}), 4.94(\mathrm{~s}, 1 \mathrm{H}), 4.14(\mathrm{q}, J$ $=7.2 \mathrm{~Hz}, 2 \mathrm{H}), 2.97(\mathrm{~s}, 2 \mathrm{H}), 2.92-2.83(\mathrm{~m}, 2 \mathrm{H}), 2.77-2.66(\mathrm{~m}, 2 \mathrm{H}), 1.71(\mathrm{~s}, 3 \mathrm{H}), 1.26(\mathrm{t}, J=7.1 \mathrm{~Hz}, 3 \mathrm{H}) .{ }^{13} \mathrm{C}$ $\operatorname{NMR}\left(101 \mathrm{MHz}, \mathrm{CDCl}_{3}\right) \delta:$ 172.1, 137.6, 136.9, 134.7, 131.6, 128.7, 118.6, 91.4, 60.8, 36.79, 29.5, 25.3, 24.8, $14.2 \mathrm{ppm}$. HRMS (ESI-TOF) m/z : $[\mathrm{M}+\mathrm{Na}]^{+} \mathrm{Calcd}$ for $\mathrm{C}_{22} \mathrm{H}_{26} \mathrm{O}_{6} \mathrm{~S}_{2}=473.1068$; Found 473.1065.<smiles>C=C(C)CC1(CC(c2ccccc2)N(Cc2ccccc2)c2ccccc2)c2ccccc2S1(=O)=O</smiles>

$N, N$-dibenzyl-5-methyl-3,3-bis(phenylsulfonyl)hex-5-en-1-amine (1p): purified by flash chromatography (on silica, petroleum ether/ ethyl acetate $=20 / 1$, v/v), white solid, $1.90 \mathrm{~g}, 70 \%$ yield (for two steps); ${ }^{1} \mathrm{H}$ NMR $\left(400 \mathrm{MHz}, \mathrm{CDCl}_{3}\right) \delta: 7.90-7.83(\mathrm{~m}, 4 \mathrm{H}), 7.68-7.63(\mathrm{~m}, 2 \mathrm{H}), 7.51-7.45(\mathrm{~m}, 4 \mathrm{H}), 7.36-7.30(\mathrm{~m}, 8 \mathrm{H}), 7.27$ $-7.23(\mathrm{~m}, 2 \mathrm{H}), 4.89(\mathrm{~s}, 1 \mathrm{H}), 4.85(\mathrm{~s}, 1 \mathrm{H}), 3.59(\mathrm{~s}, 4 \mathrm{H}), 3.04-2.95(\mathrm{~m}, 2 \mathrm{H}), 2.85(\mathrm{~s}, 2 \mathrm{H}), 2.60-2.51(\mathrm{~m}, 2 \mathrm{H})$, 1.49 (s, 3H). ${ }^{13} \mathrm{C}$ NMR (101 MHz, $\left.\mathrm{CDCl}_{3}\right) \delta: 139.6,137.6,137.0,134.4,131.5,128.9,128.5,128.3,127.1$, 118.1, 92.4, 58.7, 48.8, 36.4, 28.8, 24.6 ppm. HRMS (ESI-TOF) $\mathrm{m} / \mathrm{z}:[\mathrm{M}+\mathrm{H}]^{+}$Calcd for $\mathrm{C}_{33} \mathrm{H}_{35} \mathrm{NO}_{4} \mathrm{~S}_{2}=$ 574.2086; Found 574.2080. 


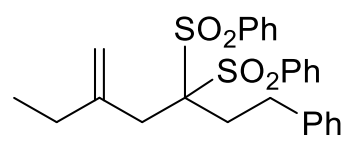

(5-methylene-1-phenylheptane-3,3-diyldisulfonyl)dibenzene (1q): purified by flash chromatography (on silica, petroleum ether/ ethyl acetate $=15 / 1, \mathrm{v} / \mathrm{v})$, white solid, $2.20 \mathrm{~g}, 46 \%$ yield (for two steps); ${ }^{1} \mathrm{H}$ NMR $(400$ $\left.\mathrm{MHz}, \mathrm{CDCl}_{3}\right) \delta: 8.13-8.07(\mathrm{~m}, 4 \mathrm{H}), 7.79-7.64(\mathrm{~m}, 2 \mathrm{H}), 7.61-7.54(\mathrm{~m}, 4 \mathrm{H}), 7.33-7.27(\mathrm{~m}, 2 \mathrm{H}), 7.24-7.18$ (m, 1H), $7.18-7.11(\mathrm{~m}, 2 \mathrm{H}), 5.21(\mathrm{~s}, 1 \mathrm{H}), 5.09(\mathrm{~s}, 1 \mathrm{H}), 3.14-3.02(\mathrm{~m}, 4 \mathrm{H}), 2.74-2.60(\mathrm{~m}, 2 \mathrm{H}), 2.03(\mathrm{q}, J=$ $7.3 \mathrm{~Hz}, 2 \mathrm{H}), 1.00(\mathrm{t}, J=7.4 \mathrm{~Hz}, 3 \mathrm{H}) .{ }^{13} \mathrm{C} \mathrm{NMR}\left(101 \mathrm{MHz}, \mathrm{CDCl}_{3}\right) \delta: 143.3,140.6,137.1,134.6,131.6,128.7$, 128.6, 128.2 126.5, 114.9, 93.1, 34.6, 31.1, 31.0, 30.3, 12.5 ppm. HRMS (ESI-TOF) m/z : [M+Na $]^{+}$Calcd for $\mathrm{C}_{26} \mathrm{H}_{28} \mathrm{O}_{4} \mathrm{~S}_{2}=491.1327$; Found 491.1320 .

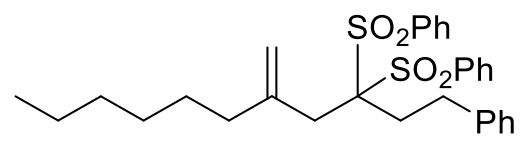

(5-methylene-1-phenylundecane-3,3-diyldisulfonyl)dibenzene (1r): purified by flash chromatography (on silica, petroleum ether/ ethyl acetate $=20 / 1$, v/v), white solid, $1.70 \mathrm{~g}, 32 \%$ yield (for two steps); ${ }^{1} \mathrm{H}$ NMR $(400$ $\left.\mathrm{MHz}, \mathrm{CDCl}_{3}\right) \delta: 8.19-8.05(\mathrm{~m}, 4 \mathrm{H}), 7.74-7.67(\mathrm{~m}, 2 \mathrm{H}), 7.61-7.54(\mathrm{~m}, 4 \mathrm{H}), 7.32-7.27(\mathrm{~m}, 2 \mathrm{H}), 7.25-7.19$ (m, 1H), $7.17-7.13(\mathrm{~m}, 2 \mathrm{H}), 5.22(\mathrm{~s}, 1 \mathrm{H}), 5.08(\mathrm{~s}, 1 \mathrm{H}), 3.13-3.02(\mathrm{~m}, 4 \mathrm{H}), 2.73-2.61(\mathrm{~m}, 2 \mathrm{H}), 2.03-1.94$ $(\mathrm{m}, 2 \mathrm{H}), 1.42-1.33(\mathrm{~m}, 2 \mathrm{H}), 1.30-1.21(\mathrm{~m}, 6 \mathrm{H}), 0.88(\mathrm{t}, J=6.9 \mathrm{~Hz}, 3 \mathrm{H}) .{ }^{13} \mathrm{C} \mathrm{NMR}\left(101 \mathrm{MHz}, \mathrm{CDCl}_{3}\right) \delta$ : 141.9, 140.6, 137.1, 134.6, 131.6, 128.7, 128.6, 128.2, 126.5, 115.9, 93.2, 38.6, 34.3, 31.7, 30.9, 30.3, 28.9, 28.0, 22.6, $14.1 \mathrm{ppm}$. HRMS (ESI-TOF) m/z : [M+Na] $]^{+}$Calcd for $\mathrm{C}_{30} \mathrm{H}_{36} \mathrm{O}_{4} \mathrm{~S}_{2}=547.1953$; Found 547.1952.<smiles>C=C(C)CC1(CC(Oc2ccccc2)c2ccccc2)SS(=O)(=O)c2ccccc21</smiles>

(5-methyl-1-phenoxyhex-5-ene-3,3-diyldisulfonyl)dibenzene (1s): purified by flash chromatography (on silica, petroleum ether/ ethyl acetate $=10 / 1$, v/v), white solid, $1.80 \mathrm{~g}, 32 \%$ yield (for two steps); ${ }^{1} \mathrm{H}$ NMR $(400$ $\left.\mathrm{MHz}, \mathrm{CDCl}_{3}\right) \delta: 8.06(\mathrm{~d}, J=8.1 \mathrm{~Hz}, 4 \mathrm{H}), 7.71(\mathrm{t}, J=7.5 \mathrm{~Hz}, 2 \mathrm{H}), 7.58(\mathrm{t}, J=7.9 \mathrm{~Hz}, 4 \mathrm{H}), 7.31-7.24(\mathrm{~m}, 2 \mathrm{H})$, $6.96(\mathrm{t}, J=7.4 \mathrm{~Hz}, 1 \mathrm{H}), 6.88(\mathrm{~d}, J=8.7 \mathrm{~Hz}, 2 \mathrm{H}), 5.01(\mathrm{~s}, 1 \mathrm{H}), 4.95(\mathrm{~s}, 1 \mathrm{H}), 4.44-4.35(\mathrm{~m}, 2 \mathrm{H}), 3.06(\mathrm{~s}, 2 \mathrm{H})$, $2.90-2.82(\mathrm{~m}, 2 \mathrm{H}), 1.77$ (s, 3H). ${ }^{13} \mathrm{C}$ NMR $\left(101 \mathrm{MHz}, \mathrm{CDCl}_{3}\right) \delta: 158.4,137.8,136.9,134.8,131.6,129.6$, 128.8, 121.2, 119.2, 114.7, 91.2, 63.5, 37.4, 30.1, 24.8 ppm. HRMS (ESI-TOF) m/z : [M+Na $]^{+}$Calcd for $\mathrm{C}_{25} \mathrm{H}_{26} \mathrm{O}_{5} \mathrm{~S}_{2}=493.1119 ;$ Found 493.1114 . 


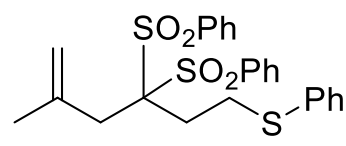

(5-methyl-3,3-bis(phenylsulfonyl)hex-5-en-1-yl)(phenyl)sulfane (1t): purified by flash chromatography (on silica, petroleum ether/ ethyl acetate $=10 / 1, \mathrm{v} / \mathrm{v})$, white solid, $2.40 \mathrm{~g}, 60 \%$ yield (for two steps); ${ }^{1} \mathrm{H}$ NMR $(400$ $\left.\mathrm{MHz}, \mathrm{CDCl}_{3}\right) \delta: 7.96(\mathrm{~d}, J=7.4 \mathrm{~Hz}, 4 \mathrm{H}), 7.68(\mathrm{t}, J=7.5 \mathrm{~Hz}, 2 \mathrm{H}), 7.53(\mathrm{t}, J=7.9 \mathrm{~Hz}, 4 \mathrm{H}), 7.47-7.35(\mathrm{~m}, 2 \mathrm{H})$, $7.35-7.22(\mathrm{~m}, 3 \mathrm{H}), 4.93(\mathrm{~s}, 1 \mathrm{H}), 4.83(\mathrm{~s}, 1 \mathrm{H}), 3.38-3.27(\mathrm{~m}, 2 \mathrm{H}), 2.94(\mathrm{~s}, 2 \mathrm{H}), 2.70-2.60(\mathrm{~m}, 2 \mathrm{H}), 1.62(\mathrm{~s}$, 3H). ${ }^{13} \mathrm{C} \mathrm{NMR}\left(101 \mathrm{MHz}, \mathrm{CDCl}_{3}\right) \delta: 137.5,136.8,134.6,134.4,131.5,131.5,129.1,128.6,127.2,118.4,91.9$, 36.6, 31.0, 29.7, $24.8 \mathrm{ppm}$. HRMS (ESI-TOF) $\mathrm{m} / \mathrm{z}:[\mathrm{M}+\mathrm{Na}]^{+}$Calcd for $\mathrm{C}_{25} \mathrm{H}_{26} \mathrm{O}_{4} \mathrm{~S}_{3}=509.0891$; Found 509.0887.<smiles>C=C(C)CCCCc1ccccc1</smiles>

(5-methylhex-5-en-1-yl)benzene (1u, known compound $\left.{ }^{3}\right)$ : purified by flash chromatography (on silica, petroleum ether), colorless liquid, $691 \mathrm{mg}, 69 \%$ yield; ${ }^{1} \mathrm{H}$ NMR (400 MHz, $\left.\mathrm{CDCl}_{3}\right) \delta: 7.32-7.21(\mathrm{~m}, 2 \mathrm{H}), 7.22$ $-7.07(\mathrm{~m}, 3 \mathrm{H}), 4.76-4.58(\mathrm{~m}, 2 \mathrm{H}), 2.68-2.56(\mathrm{~m}, 2 \mathrm{H}), 2.04(\mathrm{t}, \mathrm{J}=7.5 \mathrm{~Hz}, 2 \mathrm{H}), 1.69(\mathrm{~s}, 3 \mathrm{H}), 1.65-1.57(\mathrm{~m}$, 2H), $1.52-1.44(\mathrm{~m}, 2 \mathrm{H}) .{ }^{13} \mathrm{C} \mathrm{NMR}\left(101 \mathrm{MHz}, \mathrm{CDCl}_{3}\right) \delta: 146.0,142.8,128.4,128.3,125.7,109.9,37.7,35.9$, 31.1, 27.2, 22.4 ppm. 


\section{General Procedure for asymmetric hydroformylation}

\section{AHF procedure for substrate scope.}

In an argon-filled glovebox, to a 5-mL vial equipped with a magnetic bar was added (S,S)-DTB-YanPhos $(0.03 \mathrm{mmol})$ and $\mathrm{Rh}(\mathrm{acac})(\mathrm{CO})_{2}(0.015 \mathrm{mmol}$ in $0.25 \mathrm{~mL}$ toluene $)$. After stirring for $10 \mathrm{~min}$, to the mixture was charged with terminal olefin $1(0.5 \mathrm{mmol})$. This vial was transferred into an autoclave. The autoclave was pressurized with carbon monoxide $(2.5$ bar $)$ and hydrogen $(2.5$ bar) sequentially. The reaction mixture was stirred at $80^{\circ} \mathrm{C}$ (oil bath) for $72 \mathrm{~h}$. The reaction was then cooled to room temperature and the synthetic gas was carefully released in a well-ventilated hood. The solution was concentrated in vacuo and the aldehyde was purified by flash chromatography (on silica, petroleum ether/ ethyl acetate). The enantiomeric excesses of 2a2u were determined HPLC. The absolute configuration of $\mathbf{2 a}$ was determined by XRD after condensation with 2,4-dinitrophenylhydrazine; configuration of $\mathbf{2 b} \mathbf{-} \mathbf{2} \mathbf{u}$ were assigned by analogy.

Table S1. Results for ligand screening:

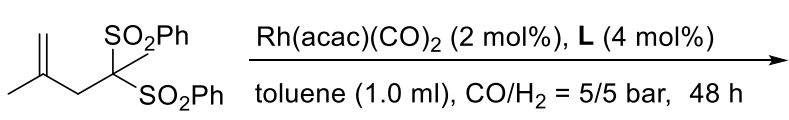

1a, $0.25 \mathrm{mmol}$

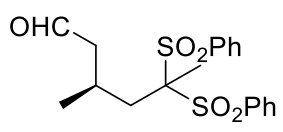

$2 a$<smiles>c1ccc(C2CC[C@@H](c3ccccc3)P2CCCP2[C@H](c3ccccc3)CC[PH2+]2c2ccccc2)cc1</smiles>

$(S, S)-P h-B P E$

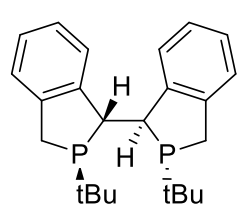

(Rc,Sp)-DuanPhos<smiles>CC(C)(C)P(=O)(O)c1nc2ccccc2nc1P(C)(C)(C)C</smiles>

$(R, R)$-QuinoxP*<smiles></smiles>

(S)-BINAP

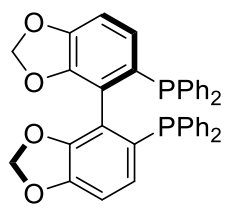

(S)-Segphos

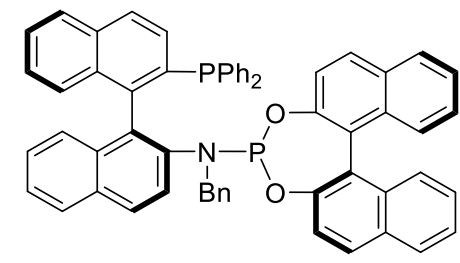

$(S, R)$-YanPhos

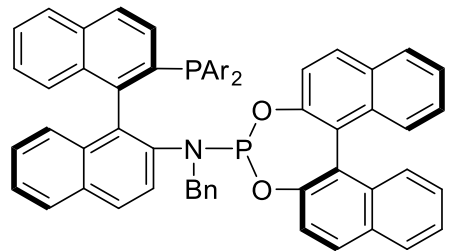

$(R, R)$-DTBM-YanPhos $\mathrm{Ar}=4-\mathrm{MeO}-3,5-{ }^{\mathrm{t}} \mathrm{Bu}-\mathrm{C}_{6} \mathrm{H}_{2}$

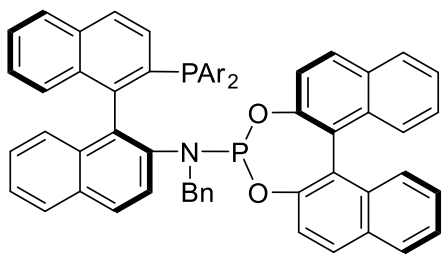

$(S, S)$-DTB-YanPhos $\mathrm{Ar}=3,5-{ }^{\mathrm{t}} \mathrm{Bu}-\mathrm{C}_{6} \mathrm{H}_{3}$

\begin{tabular}{lllll}
\hline entry & ligand & $\begin{array}{l}\text { conv. } \\
(\%)\end{array}$ & $\begin{array}{l}\mathrm{T} \\
\left({ }^{\circ} \mathrm{C}\right)\end{array}$ & $\begin{array}{l}\text { ee } \\
(\%)\end{array}$ \\
\hline 1 & $(S, S)$-Ph-BPE & 6 & 100 & 65 \\
2 & $(R \mathrm{c}, S \mathrm{p})$-DuanPhos & 16 & 100 & 15 \\
\hline
\end{tabular}




\begin{tabular}{lllll}
\hline 3 & $(R, R)$-QuinoxP* & $<1$ & 100 & - \\
4 & $(S)$-BINAP & $<1$ & 100 & - \\
5 & $(S)$-Segphos & $<1$ & 100 & - \\
6 & $(S, R)$-YanPhos & 28 & 100 & 82 \\
7 & $(R, R)$-DTBM-YanPhos & 52 & 100 & 84 \\
8 & $(S, S)$-DTB-YanPhos & 48 & 100 & 84 \\
9 & $(R, R)$-DTBM-YanPhos & 19 & 80 & 89 \\
10 & $(S, S)$-DTB-YanPhos & 21 & 80 & 90 \\
\hline
\end{tabular}

${ }^{a}$ Conditions: $1 \mathrm{a} / \mathrm{Rh}(\mathrm{acac})(\mathrm{CO})_{2} /$ ligand $=50 / 1 / 2,[1 \mathrm{a}]=0.25 \mathrm{M}, 1 \mathrm{~mL}$ toluene, the conversion was analyzed by ${ }^{1} \mathrm{H}$ NMR of the crude product, ee was determined by HPLC on a chiral stationary phase.

\section{AHF procedure for larger-scale reaction (Table 1 entry 8).}

In an argon-filled glovebox, to a 5-mL vial equipped with a magnetic bar was added $(S, S)$-DTB-YanPhos $(0.06 \mathrm{mmol})$ and $\mathrm{Rh}(\mathrm{acac})(\mathrm{CO})_{2}(0.03 \mathrm{mmol}$ in $0.5 \mathrm{~mL}$ toluene). After stirring for $10 \mathrm{~min}$, one aliquot of the homogeneous solution of catalyst $(1.0 \% \mathrm{Rh})$ was transferred to a 5-mL score-break ampule which was charged with terminal olefin 1a $(1.2 \mathrm{mmol})$. This vial was transferred into an autoclave. The autoclave was pressurized with carbon monoxide ( 2.5 bar) and hydrogen $\left(2.5\right.$ bar) sequentially. The reaction mixture was stirred at $80{ }^{\circ} \mathrm{C}$ (oil bath) for $120 \mathrm{~h}$. The reaction was then cooled to room temperature and the synthetic gas was carefully released in a well-ventilated hood. The solution was concentrated in vacuo and the aldehyde was purified by flash chromatography (on silica, petroleum ether/ ethyl acetate $=2 / 1, \mathrm{v} / \mathrm{v}$ ). The enantiomeric excess of $2 \mathbf{a}$ was determined HPLC. Results: 378 mg 2a, 80\%, 90\% ee. 


\section{Characterization data for products}<smiles>[M]C(CC(C)CC=O)(OS(=O)(=O)c1ccccc1)S(=O)(=O)O</smiles>

(S)-3-methyl-5,5-bis(phenylsulfonyl)hexanal (2a): purified by flash chromatography (on silica, petroleum ether/ ethyl acetate $=2 / 1, \mathrm{v} / \mathrm{v})$, white solid, $191 \mathrm{mg}, 97 \%$ yield; $91 \%$ ee; $[\alpha]_{\mathrm{D}}{ }^{24}=+15.4\left(\mathrm{c}=3.0, \mathrm{CH}_{3} \mathrm{CN}\right)$; The enantiomeric excess was determined by HPLC on Chiralpak AS-3 column, hexane: isopropanol = 85:15; flow rate $=1.0 \mathrm{~mL} / \mathrm{min} ; \mathrm{UV}$ detection at $220 \mathrm{~nm} ; \mathrm{t}_{\mathrm{R}}=51.6 \mathrm{~min}$ (major), $62.5 \min$ (minor). ${ }^{1} \mathrm{H} \mathrm{NMR}(400 \mathrm{MHz}$, $\left.\mathrm{CDCl}_{3}\right) \delta: 9.80-9.55(\mathrm{~m}, 1 \mathrm{H}), 8.05-7.95(\mathrm{~m}, 4 \mathrm{H}), 7.77-7.68(\mathrm{~m}, 2 \mathrm{H}), 7.60(\mathrm{t}, J=7.7 \mathrm{~Hz}, 4 \mathrm{H}), 2.69-2.59$ (m, 1H), $2.58-2.51(\mathrm{~m}, 1 \mathrm{H}), 2.39-2.31(\mathrm{~m}, 1 \mathrm{H}), 2.31-2.24(\mathrm{~m}, 1 \mathrm{H}), 2.12-2.05(\mathrm{~m}, 1 \mathrm{H}), 1.83(\mathrm{~s}, 3 \mathrm{H}), 1.02$ $(\mathrm{d}, J=6.7 \mathrm{~Hz}, 3 \mathrm{H}) .{ }^{13} \mathrm{C} \mathrm{NMR}\left(101 \mathrm{MHz}, \mathrm{CDCl}_{3}\right) \delta: 201.4,136.3,136.0,134.8,131.3,131.3,128.8,128.8,87.9$, 52.2, 37.6, 24.2, 22.7, 16.5 ppm. HRMS (ESI-TOF) $\mathrm{m} / \mathrm{z}:[\mathrm{M}+\mathrm{Na}]^{+}$Calcd for $\mathrm{C}_{19} \mathrm{H}_{22} \mathrm{O}_{5} \mathrm{~S}_{2}=417.0806$; Found 417.0800.

Product 2a was condensed with 2,4-dinitrophenylhydrazine, and the resulting compound crystalized in dichloromethane and hexane (by evaporation of solvent). CCDC No. 1993523.

Displacement ellipsoids are drawn at the $50 \%$ probability level. 


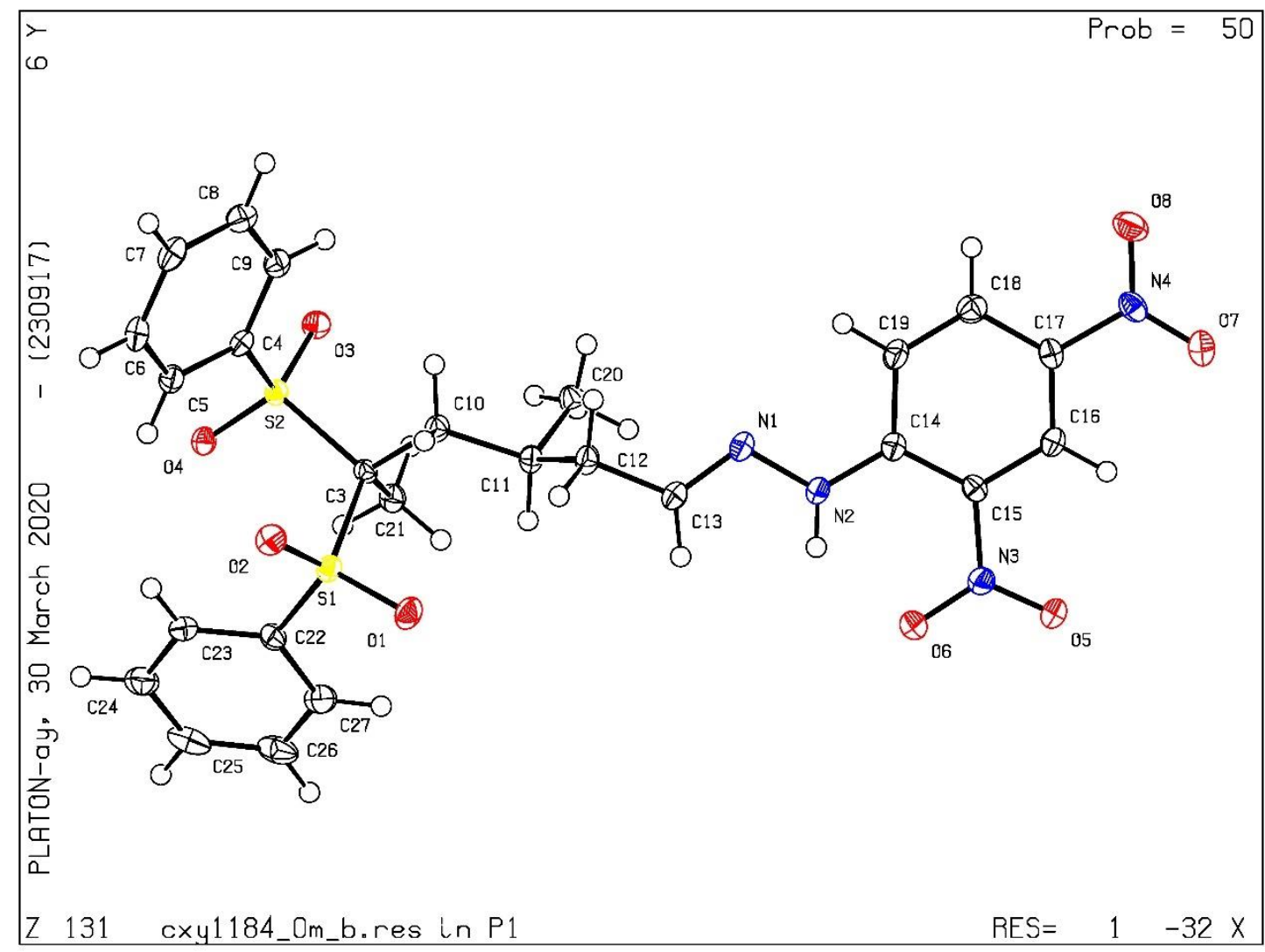

Table S1 Crystal data and structure refinement.

Identification code

Empirical formula

Formula weight

Temperature/K

Crystal system

Space group

$\mathrm{a} / \AA$

$\mathrm{b} / \AA$

$\mathrm{c} / \AA$

$\alpha /{ }^{\circ}$

$\beta /{ }^{\circ}$

$\gamma /{ }^{\circ}$

Volume $/ \AA^{3}$

$\mathrm{Z}$

$\rho_{\text {calc }} \mathrm{g} / \mathrm{cm}^{3}$

$\mu / \mathrm{mm}^{-1}$

$\mathrm{F}(000)$ cxy1184_0m

$\mathrm{C}_{26} \mathrm{H}_{28} \mathrm{Cl}_{2} \mathrm{~N}_{4} \mathrm{O}_{8} \mathrm{~S}_{2}$

659.54

100

triclinic

P1

9.6736(6)

11.1735(7)

15.1100(9)

71.190(2)

71.605(2)

78.783(2)

1459.14(16)

2

1.501

0.421

684.0 


$\begin{array}{ll}\text { Crystal size/mm } & 0.41 \times 0.38 \times 0.36 \\ \text { Radiation } & \operatorname{MoK} \alpha(\lambda=0.71073) \\ 2 \Theta \text { range for data collection } /{ }^{3} & 4.462 \text { to } 61.272 \\ \text { Index ranges } & -13 \leq \mathrm{h} \leq 13,-15 \leq \mathrm{k} \leq 16,-21 \leq 1 \leq 21 \\ \text { Reflections collected } & 60713 \\ \text { Independent reflections } & 17890\left[\mathrm{R}_{\text {int }}=0.0341, \mathrm{R}_{\text {sigma }}=0.0349\right] \\ \text { Data/restraints/parameters } & 17890 / 3 / 761 \\ \text { Goodness-of-fit on } \mathrm{F}^{2} & 1.015 \\ \text { Final R indexes [I }>=2 \sigma(\mathrm{I})] & \mathrm{R}_{1}=0.0393, \mathrm{wR}_{2}=0.0887 \\ \text { Final R indexes [all data] } & \mathrm{R}_{1}=0.0495, \mathrm{wR}_{2}=0.0945 \\ \text { Largest diff. peak/hole / e } \AA^{-3} 1.04 /-1.12 \\ \text { Flack parameter } & -0.008(16)\end{array}$<smiles>CCC(CC(C)CC=O)(OSc1ccccc1)Oc1ccccc1</smiles>

(S)-3-methyl-5,5-bis(phenylsulfonyl)heptanal (2b): purified by flash chromatography (on silica, petroleum ether/ ethyl acetate $=2 / 1, v / v)$, colorless oil, $178 \mathrm{mg}, 87 \%$ yield; $96 \%$ ee; $[\alpha]_{\mathrm{D}}^{24}=+25.4\left(\mathrm{c}=3.0, \mathrm{CH}_{3} \mathrm{CN}\right)$; The enantiomeric excess was determined by HPLC on Chiralpak AS-3 column, hexane: isopropanol = 90:10; flow rate $=1.0 \mathrm{~mL} / \mathrm{min} ; \mathrm{UV}$ detection at $220 \mathrm{~nm} ; \mathrm{t}_{\mathrm{R}}=55.3 \mathrm{~min}$ (major), $60.8 \mathrm{~min}$ (minor). ${ }^{1} \mathrm{H} \mathrm{NMR} \mathrm{(400} \mathrm{MHz}$, $\left.\mathrm{CDCl}_{3}\right) \delta: 9.72-9.67(\mathrm{~m}, 1 \mathrm{H}), 8.05-7.95(\mathrm{~m}, 4 \mathrm{H}), 7.76-7.66(\mathrm{~m}, 2 \mathrm{H}), 7.58(\mathrm{t}, J=7.7 \mathrm{~Hz}, 4 \mathrm{H}), 2.83-2.71$ $(\mathrm{m}, 2 \mathrm{H}), 2.51-2.29(\mathrm{~m}, 4 \mathrm{H}), 2.25-2.15(\mathrm{~m}, 1 \mathrm{H}), 1.16(\mathrm{t}, J=7.4 \mathrm{~Hz}, 3 \mathrm{H}), 1.09(\mathrm{~d}, J=6.7 \mathrm{~Hz}, 3 \mathrm{H}) .{ }^{13} \mathrm{C} \mathrm{NMR}$ $\left(101 \mathrm{MHz}, \mathrm{CDCl}_{3}\right)$ \&: 201.6, 137.8, 137.6, 134.6, 134.6, 131.2, 128.7, 93.2, 52.4, 36.6, 23.9, 23.8, 23.0, 9.4 ppm. HRMS (ESI-TOF) m/z : [M+Na] ${ }^{+}$Calcd for $\mathrm{C}_{20} \mathrm{H}_{24} \mathrm{O}_{5} \mathrm{~S}_{2}=431.0963$; Found 431.0959 .

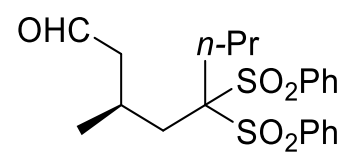

(S)-3-methyl-5,5-bis(phenylsulfonyl)octanal (2c): purified by flash chromatography (on silica, petroleum ether/ ethyl acetate $=4 / 1, \mathrm{v} / \mathrm{v})$, colorless oil, $182 \mathrm{mg}, 86 \%$ yield; $94 \%$ ee; $[\alpha]_{\mathrm{D}}{ }^{24}=+20.1\left(\mathrm{c}=3.0, \mathrm{CH}_{3} \mathrm{CN}\right)$; The enantiomeric excess was determined by HPLC on Chiralpak AS-3 column, hexane: isopropanol = 90:10; flow rate $=1.0 \mathrm{~mL} / \mathrm{min} ; \mathrm{UV}$ detection at $220 \mathrm{~nm} ; \mathrm{t}_{\mathrm{R}}=37.1 \mathrm{~min}$ (major), $42.4 \min$ (minor). ${ }^{1} \mathrm{H} \mathrm{NMR}(600 \mathrm{MHz}$, $\left.\mathrm{CDCl}_{3}\right) \delta: 9.75-9.64(\mathrm{~m}, 1 \mathrm{H}), 8.03-7.96(\mathrm{~m}, 4 \mathrm{H}), 7.73-7.68(\mathrm{~m}, 2 \mathrm{H}), 7.61-7.56(\mathrm{~m}, 4 \mathrm{H}), 2.81-2.71(\mathrm{~m}$, 2H), $2.40-2.33(\mathrm{~m}, 2 \mathrm{H}), 2.30-2.18(\mathrm{~m}, 3 \mathrm{H}), 1.63-1.57(\mathrm{~m}, 2 \mathrm{H}), 1.09$ (d, J = 6.7 Hz, 3H), 0.89 (t, J = 7.3 Hz, 
3H). ${ }^{13} \mathrm{C}$ NMR $\left(151 \mathrm{MHz}, \mathrm{CDCl}_{3}\right) \delta: 201.5,137.9,137.6,134.6,134.5,131.2,128.7,93.3,52.3,37.0,32.8$, 23.9, 22.9, 17.8, 14.6 ppm. HRMS (ESI-TOF) m/z : $[\mathrm{M}+\mathrm{Na}]^{+}$Calcd for $\mathrm{C}_{21} \mathrm{H}_{26} \mathrm{O}_{5} \mathrm{~S}_{2}=445.1119$; Found 445.1113.

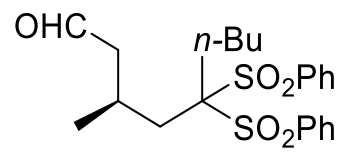

(S)-3-methyl-5,5-bis(phenylsulfonyl)nonanal (2d): purified by flash chromatography (on silica, petroleum ether/ ethyl acetate $=4 / 1, \mathrm{v} / \mathrm{v})$, colorless oil, $197 \mathrm{mg}$, $90 \%$ yield; $95 \%$ ee; $[\alpha]_{\mathrm{D}}^{24}=+18.5\left(\mathrm{c}=3.0, \mathrm{CH}_{3} \mathrm{CN}\right)$; The enantiomeric excess was determined by HPLC on Chiralpak AS-3 column, hexane: isopropanol = 90:10; flow rate $=0.7 \mathrm{~mL} / \mathrm{min} ; \mathrm{UV}$ detection at $220 \mathrm{~nm} ; \mathrm{t}_{\mathrm{R}}=37.8 \mathrm{~min}$ (major), $41.7 \min$ (minor). ${ }^{1} \mathrm{H} \mathrm{NMR}(400 \mathrm{MHz}$, $\left.\mathrm{CDCl}_{3}\right) \delta: 9.73-9.67(\mathrm{~m}, 1 \mathrm{H}), 8.07-7.94(\mathrm{~m}, 4 \mathrm{H}), 7.75-7.67(\mathrm{~m}, 2 \mathrm{H}), 7.63-7.56(\mathrm{~m}, 4 \mathrm{H}), 2.82-2.70(\mathrm{~m}$, $2 \mathrm{H}), 2.42-2.18(\mathrm{~m}, 5 \mathrm{H}), 1.57-1.48(\mathrm{~m}, 2 \mathrm{H}), 1.29-1.25(\mathrm{~m}, 2 \mathrm{H}), 1.09(\mathrm{~d}, J=6.8 \mathrm{~Hz}, 3 \mathrm{H}), 0.87(\mathrm{t}, J=7.3 \mathrm{~Hz}$, 3H). ${ }^{13} \mathrm{C}$ NMR (101 MHz, $\left.\mathrm{CDCl}_{3}\right) \delta: 201.6,137.9,137.5,134.6,134.6,131.2,128.7,128.7,93.2,52.4,37.0$, 30.6, 26.3, 23.9, 23.5, 23.0, 13.7 ppm. HRMS (ESI-TOF) $\mathrm{m} / \mathrm{z}:[\mathrm{M}+\mathrm{Na}]^{+}$Calcd for $\mathrm{C}_{22} \mathrm{H}_{28} \mathrm{O}_{5} \mathrm{~S}_{2}=459.1276$; Found 459.1271.

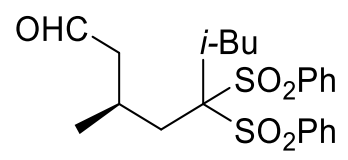

(S)-3,7-dimethyl-5,5-bis(phenylsulfonyl)octanal (2e): purified by flash chromatography (on silica, petroleum ether/ ethyl acetate $=4 / 1, \mathrm{v} / \mathrm{v})$, colorless oil, $195 \mathrm{mg}, 89 \%$ yield; $96 \%$ ee; $[\alpha]_{\mathrm{D}}{ }^{24}=+19.5\left(\mathrm{c}=4.0, \mathrm{CH}_{3} \mathrm{CN}\right)$; The enantiomeric excess was determined by HPLC on Chiralpak OJ-3 column, hexane: isopropanol = 85:15; flow rate $=1.0 \mathrm{~mL} / \mathrm{min} ; \mathrm{UV}$ detection at $220 \mathrm{~nm} ; \mathrm{t}_{\mathrm{R}}=51.6 \mathrm{~min}$ (major), $44.3 \mathrm{~min}$ (minor). ${ }^{1} \mathrm{H} \mathrm{NMR}(400 \mathrm{MHz}$, $\left.\mathrm{CDCl}_{3}\right) \delta: 9.72-9.64(\mathrm{~m}, 1 \mathrm{H}), 8.02-7.95(\mathrm{~m}, 4 \mathrm{H}), 7.73-7.67(\mathrm{~m}, 2 \mathrm{H}), 7.62-7.55(\mathrm{~m}, 4 \mathrm{H}), 2.97-2.88(\mathrm{~m}$, $1 \mathrm{H}), 2.83-2.74(\mathrm{~m}, 1 \mathrm{H}), 2.51-2.38(\mathrm{~m}, 2 \mathrm{H}), 2.33-2.27(\mathrm{~m}, 1 \mathrm{H}), 2.17(\mathrm{~d}, J=4.6 \mathrm{~Hz}, 2 \mathrm{H}), 2.14-2.05(\mathrm{~m}$, $1 \mathrm{H}), 1.13(\mathrm{~d}, J=6.8 \mathrm{~Hz}, 3 \mathrm{H}), 1.03-0.96(\mathrm{~m}, 6 \mathrm{H}) .{ }^{13} \mathrm{C} \mathrm{NMR}\left(101 \mathrm{MHz}, \mathrm{CDCl}_{3}\right) \delta: 201.9,137.9,137.9,134.6$, 134.6, 131.2, 131.1, 128.7, 128.7, 93.7, 52.4, 40.2, 37.8, 25.8, 25.7, 23.9, 23.8, 23.5 ppm. HRMS (ESI-TOF) $\mathrm{m} / \mathrm{z}:[\mathrm{M}+\mathrm{Na}]^{+}$Calcd for $\mathrm{C}_{22} \mathrm{H}_{28} \mathrm{O}_{5} \mathrm{~S}_{2}=459.1276$; Found 459.1271 .

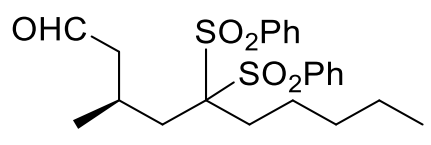

(S)-3-methyl-5,5-bis(phenylsulfonyl)decanal (2f): purified by flash chromatography (on silica, petroleum ether/ ethyl acetate $=6 / 1, \mathrm{v} / \mathrm{v})$, colorless oil, $207 \mathrm{mg}, 92 \%$ yield; $95 \%$ ee; $[\alpha]_{\mathrm{D}}{ }^{24}=+17.1\left(\mathrm{c}=2.7, \mathrm{CH}_{3} \mathrm{CN}\right)$; The 
enantiomeric excess was determined by HPLC on Chiralpak AS-3 column, hexane: isopropanol = 90:10; flow rate $=0.7 \mathrm{~mL} / \mathrm{min} ; \mathrm{UV}$ detection at $220 \mathrm{~nm} ; \mathrm{t}_{\mathrm{R}}=33.3 \mathrm{~min}$ (major), $37.1 \mathrm{~min}$ (minor). ${ }^{1} \mathrm{H} \mathrm{NMR}(400 \mathrm{MHz}$, $\left.\mathrm{CDCl}_{3}\right) \delta: 9.74-9.64(\mathrm{~m}, 1 \mathrm{H}), 8.03-7.96(\mathrm{~m}, 4 \mathrm{H}), 7.73-7.67(\mathrm{~m}, 2 \mathrm{H}), 7.61-7.54(\mathrm{~m}, 4 \mathrm{H}), 2.83-2.71(\mathrm{~m}$, 2H), $2.41-2.17(\mathrm{~m}, 5 \mathrm{H}), 1.58-1.47(\mathrm{~m}, 2 \mathrm{H}), 1.30-1.19(\mathrm{~m}, 4 \mathrm{H}), 1.09(\mathrm{~d}, J=6.7 \mathrm{~Hz}, 3 \mathrm{H}), 0.86(\mathrm{t}, J=7.0 \mathrm{~Hz}$, 3H). $\left.{ }^{13} \mathrm{C} \mathrm{NMR} \mathrm{(101} \mathrm{MHz,} \mathrm{CDCl}_{3}\right) \delta: 201.6,137.8,137.5,134.6,134.6,131.2,128.7,128.7,93.2,52.3,36.9$, 32.5, 30.8, 23.9, 23.0, 22.3, 14.0 ppm. HRMS (ESI-TOF) $\mathrm{m} / \mathrm{z}:[\mathrm{M}+\mathrm{Na}]^{+}$Calcd for $\mathrm{C}_{23} \mathrm{H}_{30} \mathrm{O}_{5} \mathrm{~S}_{2}=473.1432$; Found 473.1428.

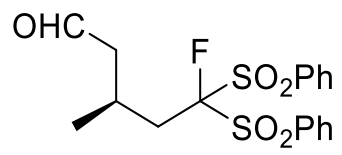

(S)-5-fluoro-3-methyl-5,5-bis(phenylsulfonyl)pentanal (2g): purified by flash chromatography (on silica, petroleum ether/ ethyl acetate $=2 / 1, \mathrm{v} / \mathrm{v})$, yelow oil, $180 \mathrm{mg}, 90 \%$ yield; $81 \%$ ee; $[\alpha]_{\mathrm{D}}{ }^{24}=+1.5\left(\mathrm{c}=2.0, \mathrm{CH}_{3} \mathrm{CN}\right)$; The enantiomeric excess was determined by HPLC on Chiralpak AS-3 column, hexane: isopropanol = 85:15; flow rate $=1.0 \mathrm{~mL} / \mathrm{min} ; \mathrm{UV}$ detection at $220 \mathrm{~nm} ; \mathrm{t}_{\mathrm{R}}=38.0 \mathrm{~min}$ (major), $42.9 \mathrm{~min}$ (minor). ${ }^{1} \mathrm{H} \mathrm{NMR}(600 \mathrm{MHz}$, $\left.\mathrm{CDCl}_{3}\right) \delta: 9.75-9.43(\mathrm{~m}, 1 \mathrm{H}), 7.93-7.90(\mathrm{~m}, 4 \mathrm{H}), 7.73(\mathrm{t}, J=7.5 \mathrm{~Hz}, 2 \mathrm{H}), 7.57(\mathrm{t}, J=7.9 \mathrm{~Hz}, 4 \mathrm{H}), 2.51-$ $2.40(\mathrm{~m}, 4 \mathrm{H}), 2.26-2.21(\mathrm{~m}, 1 \mathrm{H}), 0.89(\mathrm{~d}, J=6.1 \mathrm{~Hz}, 3 \mathrm{H}) .{ }^{13} \mathrm{C} \mathrm{NMR}\left(151 \mathrm{MHz}, \mathrm{CDCl}_{3}\right) \delta: 200.9,135.4,134.8$, 130.9, 129.1, $115.8(\mathrm{~d}, J=269.3 \mathrm{~Hz}), 50.6,35.4(\mathrm{~d}, J=16.8 \mathrm{~Hz}), 24.3(\mathrm{~d}, J=4.4 \mathrm{~Hz}), 21.3 \mathrm{ppm} .{ }^{19} \mathrm{~F}$ NMR $(565$ $\mathrm{MHz}, \mathrm{CDCl}_{3}$ ) $\delta:-147.69 \mathrm{ppm}$. HRMS (ESI-TOF) $\mathrm{m} / \mathrm{z}:[\mathrm{M}+\mathrm{Na}]^{+}$Calcd for $\mathrm{C}_{18} \mathrm{H}_{19} \mathrm{FO}_{5} \mathrm{~S}_{2}=421.0556$; Found 421.0551.

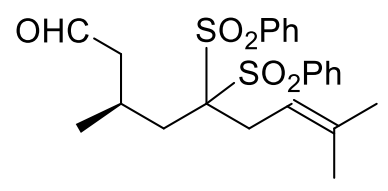

(S)-3,8-dimethyl-5,5-bis(phenylsulfonyl)non-7-enal (2h): purified by flash chromatography (on silica, petroleum ether/ ethyl acetate $=6 / 1, \mathrm{v} / \mathrm{v})$, colorless oil, $197 \mathrm{mg}, 88 \%$ yield; $96 \%$ ee; $[\alpha]_{\mathrm{D}}{ }^{24}=+9.3(\mathrm{c}=4.0$, $\mathrm{CH}_{3} \mathrm{CN}$ ); The enantiomeric excess was determined by HPLC on Chiralpak OD-H column, hexane: isopropanol $=90: 10 ;$ flow rate $=1.0 \mathrm{~mL} / \mathrm{min} ; \mathrm{UV}$ detection at $230 \mathrm{~nm} ; \mathrm{t}_{\mathrm{R}}=22.4 \mathrm{~min}$ (major), $25.0 \mathrm{~min}$ (minor). ${ }^{1} \mathrm{H} \mathrm{NMR}$ $\left(400 \mathrm{MHz}, \mathrm{CDCl}_{3}\right) \delta: 9.76-9.62(\mathrm{~m}, 1 \mathrm{H}), 8.05-7.93(\mathrm{~m}, 4 \mathrm{H}), 7.73-7.66(\mathrm{~m}, 2 \mathrm{H}), 7.61-7.52(\mathrm{~m}, 4 \mathrm{H}), 5.21$ $-5.13(\mathrm{~m}, 1 \mathrm{H}), 3.11-3.02(\mathrm{~m}, 2 \mathrm{H}), 2.86-2.76(\mathrm{~m}, 1 \mathrm{H}), 2.70(\mathrm{dd}, J=16.7,3.4 \mathrm{~Hz}, 1 \mathrm{H}), 2.42-2.31(\mathrm{~m}, 2 \mathrm{H})$, $2.21-2.14(\mathrm{~m}, 1 \mathrm{H}), 1.67(\mathrm{q}, J=1.5 \mathrm{~Hz}, 3 \mathrm{H}), 1.57(\mathrm{~d}, J=1.4 \mathrm{~Hz}, 3 \mathrm{H}), 1.05(\mathrm{~d}, J=6.7 \mathrm{~Hz}, 3 \mathrm{H}) .{ }^{13} \mathrm{C}$ NMR $(101$ $\left.\mathrm{MHz}, \mathrm{CDCl}_{3}\right) \delta: 201.6,137.5,137.2,136.4,134.6,134.5,131.4,128.6,128.5,116.1,92.5,52.1,36.0,29.0$, 
26.0, 24.0, 22.7, $18.1 \mathrm{ppm}$. HRMS (ESI-TOF) $\mathrm{m} / \mathrm{z}:[\mathrm{M}+\mathrm{Na}]^{+}$Calcd for $\mathrm{C}_{23} \mathrm{H}_{28} \mathrm{O}_{5} \mathrm{~S}_{2}=471.1276$; Found 471.1274 .

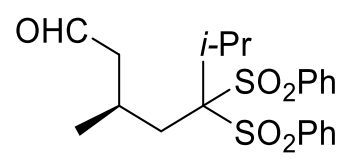

(S)-3,6-dimethyl-5,5-bis(phenylsulfonyl)heptanal (2i): purified by flash chromatography (on silica, petroleum ether/ ethyl acetate $=4 / 1, \mathrm{v} / \mathrm{v})$, colorless oil, $156 \mathrm{mg}, 74 \%$ yield; $98 \%$ ee; $[\alpha]_{\mathrm{D}}{ }^{24}=+13.3(\mathrm{c}=2.0$, $\mathrm{CH}_{3} \mathrm{CN}$ ); The enantiomeric excess was determined by HPLC on Chiralpak AS-3 column, hexane: isopropanol $=91: 9 ;$ flow rate $=0.9 \mathrm{~mL} / \mathrm{min} ; \mathrm{UV}$ detection at $220 \mathrm{~nm} ; \mathrm{t}_{\mathrm{R}}=40.8 \mathrm{~min}$ (major), $44.9 \mathrm{~min}$ (minor). ${ }^{1} \mathrm{H} \mathrm{NMR}$ $\left(400 \mathrm{MHz}, \mathrm{CDCl}_{3}\right) \delta: 9.71-9.57(\mathrm{~m}, 1 \mathrm{H}), 8.23-8.15(\mathrm{~m}, 4 \mathrm{H}), 7.74-7.68(\mathrm{~m}, 2 \mathrm{H}), 7.64-7.56(\mathrm{~m}, 4 \mathrm{H}), 3.02$ $(\mathrm{p}, J=7.0 \mathrm{~Hz}, 1 \mathrm{H}), 2.75-2.66(\mathrm{~m}, 1 \mathrm{H}), 2.58-2.52(\mathrm{~m}, 1 \mathrm{H}), 2.30-2.13(\mathrm{~m}, 3 \mathrm{H}), 1.26(\mathrm{~s}, 3 \mathrm{H}), 1.13(\mathrm{~d}, J=6.9$ $\mathrm{Hz}, 3 \mathrm{H}), 1.04(\mathrm{~d}, J=6.6 \mathrm{~Hz}, 3 \mathrm{H}) .{ }^{13} \mathrm{C} \mathrm{NMR}\left(101 \mathrm{MHz}, \mathrm{CDCl}_{3}\right) \delta: 201.3,139.8,139.4,134.5,134.5,131.8$, 131.7, 128.6, 128.6, 98.8, 52.8, 37.7, 30.7, 23.4, 23.3, 19.8, 19.7 ppm. HRMS (ESI-TOF) m/z : [M+Na $]^{+}$Calcd for $\mathrm{C}_{21} \mathrm{H}_{26} \mathrm{O}_{5} \mathrm{~S}_{2}=445.1119$; Found 445.1114 .<smiles>CC(CC=O)CC(Br)(Br)S(=O)(=O)c1ccccc1</smiles>

(S)-3-methyl-6-phenyl-5,5-bis(phenylsulfonyl)hexanal (2j): purified by flash chromatography (on silica, petroleum ether/ ethyl acetate $=4 / 1, \mathrm{v} / \mathrm{v})$, colorless oil, $170 \mathrm{mg}, 72 \%$ yield; $99 \%$ ee; $[\alpha]_{\mathrm{D}}{ }^{24}=+17.4(\mathrm{c}=2.0$, $\mathrm{CH}_{3} \mathrm{CN}$ ); The enantiomeric excess was determined by HPLC on Chiralpak AS-H column, hexane: isopropanol $=85: 15 ;$ flow rate $=1.0 \mathrm{~mL} / \mathrm{min} ; \mathrm{UV}$ detection at $220 \mathrm{~nm} ; \mathrm{t}_{\mathrm{R}}=23.5 \mathrm{~min}$ (major), $28.5 \mathrm{~min}$ (minor). ${ }^{1} \mathrm{H} \mathrm{NMR}$ $\left(400 \mathrm{MHz}, \mathrm{CDCl}_{3}\right) \delta: 9.67-9.54(\mathrm{~m}, 1 \mathrm{H}), 8.04-7.98(\mathrm{~m}, 2 \mathrm{H}), 7.79-7.76(\mathrm{~m}, 2 \mathrm{H}), 7.75-7.70(\mathrm{~m}, 1 \mathrm{H}), 7.67$ $-7.62(\mathrm{~m}, 1 \mathrm{H}), 7.61-7.56(\mathrm{~m}, 2 \mathrm{H}), 7.50-7.45(\mathrm{~m}, 2 \mathrm{H}), 7.35-7.29(\mathrm{~m}, 2 \mathrm{H}), 7.27-7.22(\mathrm{~m}, 3 \mathrm{H}), 3.86-3.72$ (m, 2H), $2.84-2.73(\mathrm{~m}, 1 \mathrm{H}), 2.72-2.64(\mathrm{~m}, 1 \mathrm{H}), 2.44(\mathrm{dd}, J=15.3,5.6 \mathrm{~Hz}, 1 \mathrm{H}), 2.29-2.10(\mathrm{~m}, 2 \mathrm{H}), 0.92$ $(\mathrm{d}, J=6.7 \mathrm{~Hz}, 3 \mathrm{H}) .{ }^{13} \mathrm{C} \mathrm{NMR}\left(101 \mathrm{MHz}, \mathrm{CDCl}_{3}\right) \delta: 201.8,137.7,137.5,134.7,134.4,133.1,132.1,131.5$, $131.2,128.7,128.6,128.5,127.8,93.5,52.1,37.8,36.5,24.1,23.0$ ppm. HRMS (ESI-TOF) m/z : [M+Na] ${ }^{+}$ Calcd for $\mathrm{C}_{25} \mathrm{H}_{26} \mathrm{O}_{5} \mathrm{~S}_{2}=493.1119$; Found 493.1118.

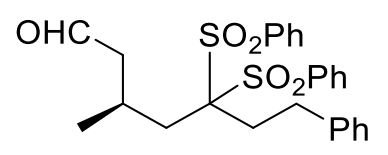

(S)-3-methyl-7-phenyl-5,5-bis(phenylsulfonyl)heptanal (2k): purified by flash chromatography (on silica, petroleum ether/ ethyl acetate $=6 / 1, \mathrm{v} / \mathrm{v})$, colorless oil, $221 \mathrm{mg}, 91 \%$ yield; $97 \%$ ee; $[\alpha]_{\mathrm{D}}{ }^{24}=+7.3(\mathrm{c}=2.5$, 
$\mathrm{CH}_{3} \mathrm{CN}$ ); The enantiomeric excess was determined by HPLC on Chiralpak AS-3 column, hexane: isopropanol $=90: 10 ;$ flow rate $=1.0 \mathrm{~mL} / \mathrm{min} ; \mathrm{UV}$ detection at $230 \mathrm{~nm} ; \mathrm{t}_{\mathrm{R}}=57.0 \mathrm{~min}$ (major), $62.9 \mathrm{~min}$ (minor). ${ }^{1} \mathrm{H} \mathrm{NMR}$ $\left(600 \mathrm{MHz}, \mathrm{CDCl}_{3}\right) \delta: 9.68(\mathrm{~d}, J=2.2 \mathrm{~Hz}, 1 \mathrm{H}), 8.03(\mathrm{t}, J=8.0 \mathrm{~Hz}, 4 \mathrm{H}), 7.74-7.68(\mathrm{~m}, 2 \mathrm{H}), 7.62-7.55(\mathrm{~m}$, 4H), $7.29(\mathrm{t}, J=7.5 \mathrm{~Hz}, 2 \mathrm{H}), 7.22(\mathrm{t}, J=7.4 \mathrm{~Hz}, 1 \mathrm{H}), 7.14-7.10(\mathrm{~m}, 2 \mathrm{H}), 2.93(\mathrm{t}, J=8.7 \mathrm{~Hz}, 2 \mathrm{H}), 2.85-2.78$ (m, 1H), $2.77-2.72(\mathrm{~m}, 1 \mathrm{H}), 2.68-2.59(\mathrm{~m}, 2 \mathrm{H}), 2.43-2.37(\mathrm{~m}, 2 \mathrm{H}), 2.31-2.26(\mathrm{~m}, 1 \mathrm{H}), 1.13(\mathrm{~d}, J=6.7$ $\mathrm{Hz}, 3 \mathrm{H}) .{ }^{13} \mathrm{C} \mathrm{NMR}\left(101 \mathrm{MHz}, \mathrm{CDCl}_{3}\right) \delta: 201.4,140.4,137.8,137.4,134.8,134.8,131.3,131.26,128.9,128.8$, 128.8, 128.2, 126.6, 92.9, 52.4, 37.4, 32.6, 30.5, 24.0, $23.2 \mathrm{ppm}$. HRMS (ESI-TOF) $\mathrm{m} / \mathrm{z}:[\mathrm{M}+\mathrm{Na}]^{+}$Calcd for $\mathrm{C}_{26} \mathrm{H}_{28} \mathrm{O}_{5} \mathrm{~S}_{2}=507.1276$; Found 507.1274.

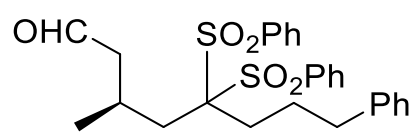

(S)-3-methyl-8-phenyl-5,5-bis(phenylsulfonyl)octanal (21): purified by flash chromatography (on silica, petroleum ether/ ethyl acetate $=8 / 1, \mathrm{v} / \mathrm{v})$, colorless oil, $228 \mathrm{mg}, 92 \%$ yield; $95 \%$ ee; $[\alpha]_{\mathrm{D}}^{24}=+12.0(\mathrm{c}=2.0$, $\mathrm{CH}_{3} \mathrm{CN}$ ); The enantiomeric excess was determined by HPLC on Chiralpak AS-3 column, hexane: isopropanol $=85: 15 ;$ flow rate $=1.0 \mathrm{~mL} / \mathrm{min} ; \mathrm{UV}$ detection at $220 \mathrm{~nm} ; \mathrm{t}_{\mathrm{R}}=30.1 \mathrm{~min}$ (major), $42.2 \mathrm{~min}$ (minor). ${ }^{1} \mathrm{H} \mathrm{NMR}$ $\left(600 \mathrm{MHz}, \mathrm{CDCl}_{3}\right)$ $\delta: 9.71-9.56(\mathrm{~m}, 1 \mathrm{H}), 8.01-7.87(\mathrm{~m}, 4 \mathrm{H}), 7.72-7.66(\mathrm{~m}, 2 \mathrm{H}), 7.61-7.52(\mathrm{~m}, 4 \mathrm{H}), 7.31$ $-7.26(\mathrm{~m}, 2 \mathrm{H}), 7.23-7.18(\mathrm{~m}, 1 \mathrm{H}), 7.12-7.07(\mathrm{~m}, 2 \mathrm{H}), 2.72-2.55(\mathrm{~m}, 4 \mathrm{H}), 2.36-2.23(\mathrm{~m}, 4 \mathrm{H}), 2.18-2.11$ (m, 1H), $2.00-1.89(\mathrm{~m}, 2 \mathrm{H}), 1.03(\mathrm{~d}, J=6.7 \mathrm{~Hz}, 3 \mathrm{H}) .{ }^{13} \mathrm{C} \mathrm{NMR}\left(101 \mathrm{MHz}, \mathrm{CDCl}_{3}\right) \delta: 201.5,140.6,137.7$, 137.4, 134.6, 134.6, 131.2 128.8, 128.7, 128.5, 126.3, 92.8, 52.3, 37.2, 36.2, 30.5, 26.0, 23.9, 23.0 ppm. HRMS (ESI-TOF) $\mathrm{m} / \mathrm{z}:[\mathrm{M}+\mathrm{Na}]^{+}$Calcd for $\mathrm{C}_{27} \mathrm{H}_{30} \mathrm{O}_{5} \mathrm{~S}_{2}=521.1432$; Found 521.1431.

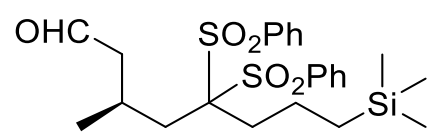

(S)-3-methyl-5,5-bis(phenylsulfonyl)-8-(trimethylsilyl)octanal (2m): purified by flash chromatography (on silica, petroleum ether/ ethyl acetate $=8 / 1, \mathrm{v} / \mathrm{v})$, colorless oil, $231 \mathrm{mg}, 94 \%$ yield; $94 \%$ ee; $[\alpha]_{\mathrm{D}}{ }^{24}=+20.5(\mathrm{c}=$ 4.0, $\mathrm{CH}_{3} \mathrm{CN}$ ); The enantiomeric excess was determined by HPLC on Chiralpak AS-3 column, hexane: isopropanol $=91: 9$; flow rate $=0.6 \mathrm{~mL} / \mathrm{min} ; \mathrm{UV}$ detection at $220 \mathrm{~nm} ; \mathrm{t}_{\mathrm{R}}=20.6 \mathrm{~min}$ (major), 24. $\min$ (minor). ${ }^{1} \mathrm{H}$ NMR $\left(600 \mathrm{MHz}, \mathrm{CDCl}_{3}\right)$ 8: $9.76(\mathrm{~d}, J=2.5 \mathrm{~Hz}, 1 \mathrm{H}), 8.10-8.00(\mathrm{~m}, 4 \mathrm{H}), 7.79-7.74(\mathrm{~m}, 2 \mathrm{H}), 7.67-7.62$ (m, 4H), $2.89-2.79(\mathrm{~m}, 2 \mathrm{H}), 2.46-2.34(\mathrm{~m}, 4 \mathrm{H}), 2.28-2.24(\mathrm{~m}, 1 \mathrm{H}), 1.58-1.50(\mathrm{~m}, 2 \mathrm{H}), 1.15(\mathrm{~d}, J=6.7$ $\mathrm{Hz}, 3 \mathrm{H}), 0.47(\mathrm{t}, J=8.5 \mathrm{~Hz}, 2 \mathrm{H}), 0.00(\mathrm{~s}, 9 \mathrm{H}) .{ }^{13} \mathrm{C} \mathrm{NMR}\left(151 \mathrm{MHz}, \mathrm{CDCl}_{3}\right) \delta: 203.2,139.7,139.4,136.3,136.2$, 
132.9, 132.9, 130.4 95.0, 54.1, 38.7, 36.3, 25.7, 24.6, 20.8, 19.6 ppm. HRMS (ESI-TOF) m/z : [M+Na $]^{+}$Calcd for $\mathrm{C}_{24} \mathrm{H}_{34} \mathrm{O}_{5} \mathrm{~S}_{2} \mathrm{Si}=517.1515$; Found 517.1512.

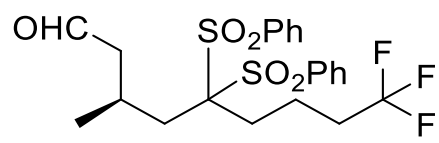

(S)-9,9,9-trifluoro-3-methyl-5,5-bis(phenylsulfonyl)nonanal (2n): purified by flash chromatography (on silica, petroleum ether/ ethyl acetate $=8 / 1$, v/v), colorless oil, $201 \mathrm{mg}, 85 \%$ yield; $94 \%$ ee; $[\alpha]_{\mathrm{D}}{ }^{24}=+15.8(\mathrm{c}=$ 2.0, $\mathrm{CH}_{3} \mathrm{CN}$ ); The enantiomeric excess was determined by HPLC on Chiralpak AS-3 column, hexane: isopropanol $=85: 15$; flow rate $=1.0 \mathrm{~mL} / \mathrm{min}$; $\mathrm{UV}$ detection at $220 \mathrm{~nm} ; \mathrm{t}_{\mathrm{R}}=22.2 \mathrm{~min}$ (major), $26.9 \mathrm{~min}$ (minor) ${ }^{1} \mathrm{H}$ NMR $\left(400 \mathrm{MHz}, \mathrm{CDCl}_{3}\right)$ ): $9.81-9.54(\mathrm{~m}, 1 \mathrm{H}), 8.03-7.97(\mathrm{~m}, 4 \mathrm{H}), 7.75-7.70(\mathrm{~m}, 2 \mathrm{H}), 7.62-7.58(\mathrm{~m}$, $4 \mathrm{H}), 2.75-2.65(\mathrm{~m}, 2 \mathrm{H}), 2.41-2.28(\mathrm{~m}, 4 \mathrm{H}), 2.20-2.15(\mathrm{~m}, 1 \mathrm{H}), 2.10-2.00(\mathrm{~m}, 2 \mathrm{H}), 1.94-1.85(\mathrm{~m}, 2 \mathrm{H})$, $1.07(\mathrm{~d}, J=6.7 \mathrm{~Hz}, 3 \mathrm{H}) .{ }^{13} \mathrm{C} \mathrm{NMR}\left(151 \mathrm{MHz}, \mathrm{CDCl}_{3}\right) \delta: 201.2,137.3,134.9,131.2,128.9,126.6(\mathrm{q}, J=276.8$ $\mathrm{Hz}), 92.4,52.4,37.4,34.1$ (q, $J=29.2 \mathrm{~Hz}), 29.9,23.8,23.0,17.4(\mathrm{q}, J=3.0 \mathrm{~Hz}) \mathrm{ppm} .{ }^{19} \mathrm{~F}$ NMR $(565 \mathrm{MHz}$, $\mathrm{CDCl}_{3}$ ) $\delta$ : -65.76 ppm. HRMS (ESI-TOF) $\mathrm{m} / \mathrm{z}:[\mathrm{M}+\mathrm{Na}]^{+}$Calcd for $\mathrm{C}_{22} \mathrm{H}_{25} \mathrm{~F}_{3} \mathrm{O}_{5} \mathrm{~S}_{2}=513.0993$; Found 513.0990.<smiles>CCOC(=O)CC(CC(C)CC=O)(S(=O)(=O)c1ccccc1)S(=O)(=O)c1ccccc1</smiles>

ethyl (S)-6-methyl-8-oxo-4,4-bis(phenylsulfonyl)octanoate (20): purified by flash chromatography (on silica, petroleum ether/ ethyl acetate $=4 / 1, \mathrm{v} / \mathrm{v})$, colorless oil, $212 \mathrm{mg}, 88 \%$ yield; $97 \%$ ee; $[\alpha]_{\mathrm{D}}{ }^{24}=+11.8(\mathrm{c}=4.0$, $\mathrm{CH}_{3} \mathrm{CN}$ ); The enantiomeric excess was determined by HPLC on Chiralpak AS-3 column, hexane: isopropanol $=75: 25 ;$ flow rate $=1.0 \mathrm{~mL} / \mathrm{min} ; \mathrm{UV}$ detection at $220 \mathrm{~nm} ; \mathrm{t}_{\mathrm{R}}=25.1 \mathrm{~min}$ (major), $30.5 \mathrm{~min}$ (minor). ${ }^{1} \mathrm{H} \mathrm{NMR}$ $\left(400 \mathrm{MHz}, \mathrm{CDCl}_{3}\right) \delta: 9.70-9.58(\mathrm{~m}, 1 \mathrm{H}), 8.06-7.98(\mathrm{~m}, 4 \mathrm{H}), 7.74-7.69(\mathrm{~m}, 2 \mathrm{H}), 7.62-7.57(\mathrm{~m}, 4 \mathrm{H}), 4.13$ $(\mathrm{q}, J=7.1 \mathrm{~Hz}, 2 \mathrm{H}), 2.76-2.60(\mathrm{~m}, 6 \mathrm{H}), 2.39-2.24(\mathrm{~m}, 2 \mathrm{H}), 2.14-2.08(\mathrm{~m}, 1 \mathrm{H}), 1.25(\mathrm{t}, J=7.1 \mathrm{~Hz}, 3 \mathrm{H}), 1.05$ $(\mathrm{d}, J=6.8 \mathrm{~Hz}, 3 \mathrm{H}) .{ }^{13} \mathrm{C} \mathrm{NMR}\left(101 \mathrm{MHz}, \mathrm{CDCl}_{3}\right) \delta: 201.3,172.0,137.4,137.0,134.9,134.8,131.3,131.3$, 128.9, 128.9, 91.5, 60.9, 52.3, 37.9, 29.4, 25.9, 23.8, 23.1, 14.2 ppm. HRMS (ESI-TOF) m/z : [M+Na] $]^{+}$Calcd for $\mathrm{C}_{23} \mathrm{H}_{28} \mathrm{O}_{7} \mathrm{~S}_{2}=503.1174 ;$ Found 503.1171.

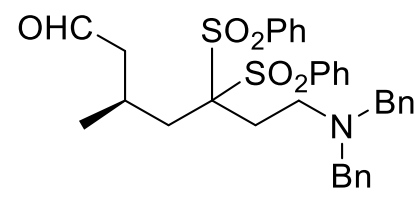

(S)-7-(dibenzylamino)-3-methyl-5,5-bis(phenylsulfonyl)heptanal (2p): purified by flash chromatography (on silica, petroleum ether/ ethyl acetate $=8 / 1$, v/v), colorless oil, $254 \mathrm{mg}, 84 \%$ yield; $86 \%$ ee; $[\alpha]_{\mathrm{D}}{ }^{24}=+7.8(\mathrm{c}$ $=4.0, \mathrm{CH}_{3} \mathrm{CN}$ ); The enantiomeric excess was determined by HPLC on Chiralpak AS-H column, hexane: 
isopropanol $=90: 10$; flow rate $=1.0 \mathrm{~mL} / \mathrm{min} ; \mathrm{UV}$ detection at $220 \mathrm{~nm} ; \mathrm{t}_{\mathrm{R}}=22.8 \mathrm{~min}$ (major), $27.5 \mathrm{~min}$ (minor). ${ }^{1} \mathrm{H}$ NMR $\left(400 \mathrm{MHz}, \mathrm{CDCl}_{3}\right)$ 8: $9.61-9.37(\mathrm{~m}, 1 \mathrm{H}), 7.88-7.81(\mathrm{~m}, 2 \mathrm{H}), 7.81-7.76(\mathrm{~m}, 2 \mathrm{H}), 7.70-7.62(\mathrm{~m}$, 2H), $7.53-7.45(\mathrm{~m}, 4 \mathrm{H}), 7.37-7.29(\mathrm{~m}, 8 \mathrm{H}), 7.27-7.22(\mathrm{~m}, 2 \mathrm{H}), 3.60(\mathrm{~d}, J=1.8 \mathrm{~Hz}, 4 \mathrm{H}), 2.95-2.81(\mathrm{~m}$, 2H), $2.53-2.43(\mathrm{~m}, 4 \mathrm{H}), 2.18(\mathrm{dd}, J=15.5,5.1 \mathrm{~Hz}, 1 \mathrm{H}), 2.11-2.03(\mathrm{~m}, 1 \mathrm{H}), 2.00-1.92(\mathrm{~m}, 1 \mathrm{H}), 0.78(\mathrm{~d}, J$ $=6.6 \mathrm{~Hz}, 3 \mathrm{H}) .{ }^{13} \mathrm{C} \mathrm{NMR}\left(101 \mathrm{MHz}, \mathrm{CDCl}_{3}\right) \delta: 201.5,139.5,137.8,137.3,134.6,134.6,131.1,131.1,128.9$, 128.8, 128.4, 127.2 92.5, 58.9, 52.0, 48.9, 37.4, 29.6, 23.8, 22.8 ppm. HRMS (ESI-TOF) m/z : [M+H] $]^{+}$Calcd for $\mathrm{C}_{34} \mathrm{H}_{37} \mathrm{NO}_{5} \mathrm{~S}_{2}=604.2191$; Found 604.2189.

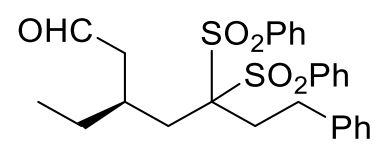

(S)-3-ethyl-7-phenyl-5,5-bis(phenylsulfonyl)heptanal (2q): purified by flash chromatography (on silica, petroleum ether/ ethyl acetate $=6 / 1, \mathrm{v} / \mathrm{v})$, colorless oil, $205 \mathrm{mg}, 82 \%$ yield; $95 \%$ ee; $[\alpha]_{\mathrm{D}}{ }^{24}=+2.5(\mathrm{c}=1.6$, $\mathrm{CH}_{3} \mathrm{CN}$ ); The enantiomeric excess was determined by HPLC on Chiralpak AS-3 column, hexane: isopropanol $=95: 5 ;$ flow rate $=1.0 \mathrm{~mL} / \mathrm{min} ; \mathrm{UV}$ detection at $205 \mathrm{~nm} ; \mathrm{t}_{\mathrm{R}}=81.1 \mathrm{~min}$ (major), $88.1 \mathrm{~min}$ (minor). ${ }^{1} \mathrm{H} \mathrm{NMR}$ $\left(600 \mathrm{MHz}, \mathrm{CDCl}_{3}\right) \delta: 9.71(\mathrm{~d}, J=2.0 \mathrm{~Hz}, 1 \mathrm{H}), 8.07-8.01(\mathrm{~m}, 4 \mathrm{H}), 7.72(\mathrm{t}, J=7.5 \mathrm{~Hz}, 2 \mathrm{H}), 7.62-7.58(\mathrm{~m}$, 4H), $7.30(\mathrm{t}, J=7.5 \mathrm{~Hz}, 2 \mathrm{H}), 7.23(\mathrm{t}, J=7.4 \mathrm{~Hz}, 1 \mathrm{H}), 7.13(\mathrm{~d}, J=7.4 \mathrm{~Hz}, 2 \mathrm{H}), 2.97-2.86(\mathrm{~m}, 2 \mathrm{H}), 2.82-2.77$ (m, 1H), $2.70-2.62(\mathrm{~m}, 3 \mathrm{H}), 2.57-2.51(\mathrm{~m}, 1 \mathrm{H}), 2.40-2.34(\mathrm{~m}, 2 \mathrm{H}), 1.61-1.54(\mathrm{~m}, 1 \mathrm{H}), 1.53-1.45(\mathrm{~m}$, 1H), $0.85(\mathrm{t}, J=7.4 \mathrm{~Hz}, 3 \mathrm{H}) .{ }^{13} \mathrm{C} \mathrm{NMR}\left(101 \mathrm{MHz}, \mathrm{CDCl}_{3}\right) \delta: 201.8,140.5,137.9,137.5,134.8,131.3,131.3$, 128.9, 128.8, 128.2 126.6, 92.8, 48.5, 36.0, 32.8, 30.5, 29.5, 29.3, 10.7 ppm. HRMS (ESI-TOF) m/z : [M+Na $]^{+}$ Calcd for $\mathrm{C}_{27} \mathrm{H}_{30} \mathrm{O}_{5} \mathrm{~S}_{2}=521.1432$; Found 521.1424.

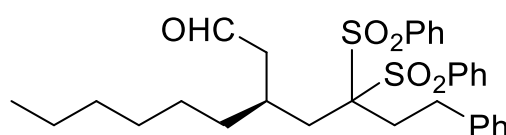

(S)-3-(4-phenyl-2,2-bis(phenylsulfonyl)butyl)nonanal (2r): purified by flash chromatography (on silica, petroleum ether/ ethyl acetate $=10 / 1, \mathrm{v} / \mathrm{v})$, colorless oil, $211 \mathrm{mg}, 76 \%$ yield; $94 \%$ ee; $[\alpha]_{\mathrm{D}}{ }^{24}=+2.8(\mathrm{c}=1.5$, $\mathrm{CH}_{3} \mathrm{CN}$ ); The enantiomeric excess was determined by HPLC on Chiralpak OD-H column, hexane: isopropanol $=90: 10 ;$ flow rate $=1.0 \mathrm{~mL} / \mathrm{min} ; \mathrm{UV}$ detection at $210 \mathrm{~nm} ; \mathrm{t}_{\mathrm{R}}=21.6 \mathrm{~min}$ (major), $26.9 \mathrm{~min}$ (minor). ${ }^{1} \mathrm{H} \mathrm{NMR}$ $\left(400 \mathrm{MHz}, \mathrm{CDCl}_{3}\right) \delta: 9.78-9.59(\mathrm{~m}, 1 \mathrm{H}), 8.08-8.01(\mathrm{~m}, 4 \mathrm{H}), 7.75-7.70(\mathrm{~m}, 2 \mathrm{H}), 7.63-7.57(\mathrm{~m}, 4 \mathrm{H}), 7.30$ $(\mathrm{dd}, J=8.1,6.6 \mathrm{~Hz}, 2 \mathrm{H}), 7.25-7.20(\mathrm{~m}, 1 \mathrm{H}), 7.15-7.10(\mathrm{~m}, 2 \mathrm{H}), 2.98-2.85(\mathrm{~m}, 2 \mathrm{H}), 2.83-2.75(\mathrm{~m}, 1 \mathrm{H})$, $2.72-2.60(\mathrm{~m}, 3 \mathrm{H}), 2.58-2.50(\mathrm{~m}, 1 \mathrm{H}), 2.44-2.32(\mathrm{~m}, 2 \mathrm{H}), 1.52-1.39(\mathrm{~m}, 2 \mathrm{H}), 1.25-1.13(\mathrm{~m}, 8 \mathrm{H}), 0.86$ $(\mathrm{t}, J=6.9 \mathrm{~Hz}, 3 \mathrm{H}) .{ }^{13} \mathrm{C} \mathrm{NMR}\left(101 \mathrm{MHz}, \mathrm{CDCl}_{3}\right) \delta: 201.9,140.5,138.0,137.5,134.7,134.7,131.3,131.3,128.8$, 
$128.8,128.2,126.6,92.8,49.1,37.0,36.4,32.7,31.7,30.5,29.3,28.6,26.4,22.6,14.1$ ppm. HRMS (ESI-TOF) $\mathrm{m} / \mathrm{z}:[\mathrm{M}+\mathrm{Na}]^{+}$Calcd for $\mathrm{C}_{31} \mathrm{H}_{38} \mathrm{O}_{5} \mathrm{~S}_{2}=577.2058 ;$ Found 577.2050.

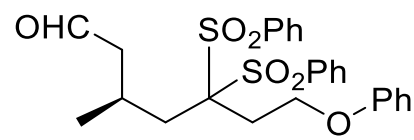

(S)-3-methyl-7-phenoxy-5,5-bis(phenylsulfonyl)heptanal (2s): purified by flash chromatography (on silica, petroleum ether/ ethyl acetate $=6 / 1, \mathrm{v} / \mathrm{v})$, colorless oil, $207 \mathrm{mg}, 88 \%$ yield; $96 \%$ ee; $[\alpha]_{\mathrm{D}}{ }^{24}=+11.8(\mathrm{c}=2.0$, $\mathrm{CH}_{3} \mathrm{CN}$ ); The enantiomeric excess was determined by HPLC on Chiralpak AS-3 column, hexane: isopropanol $=85: 15$; flow rate $=1.0 \mathrm{~mL} / \mathrm{min} ; \mathrm{UV}$ detection at $220 \mathrm{~nm} ; \mathrm{t}_{\mathrm{R}}=41.3 \mathrm{~min}$ (major), $49.4 \mathrm{~min}$ (minor). ${ }^{1} \mathrm{H} \mathrm{NMR}$ $\left(400 \mathrm{MHz}, \mathrm{CDCl}_{3}\right)$ 8: $9.74-9.54(\mathrm{~m}, 1 \mathrm{H}), 8.06-7.99(\mathrm{~m}, 4 \mathrm{H}), 7.72-7.65(\mathrm{~m}, 2 \mathrm{H}), 7.60-7.54(\mathrm{~m}, 4 \mathrm{H}), 7.29$ $-7.23(\mathrm{~m}, 2 \mathrm{H}), 6.98-6.93(\mathrm{~m}, 1 \mathrm{H}), 6.85-6.81(\mathrm{~m}, 2 \mathrm{H}), 4.25(\mathrm{q}, J=6.8 \mathrm{~Hz}, 2 \mathrm{H}), 2.85(\mathrm{t}, J=7.3 \mathrm{~Hz}, 2 \mathrm{H}), 2.79$ $-2.71(\mathrm{~m}, 1 \mathrm{H}), 2.71-2.64(\mathrm{~m}, 1 \mathrm{H}), 2.39-2.31(\mathrm{~m}, 2 \mathrm{H}), 2.26-2.20(\mathrm{~m}, 1 \mathrm{H}), 1.06(\mathrm{~d}, J=6.7 \mathrm{~Hz}, 3 \mathrm{H}) .{ }^{13} \mathrm{C}$ $\operatorname{NMR}\left(101 \mathrm{MHz}, \mathrm{CDCl}_{3}\right) \delta: 201.4,158.2,137.2,136.8,134.9,134.9,131.3,131.2,129.6,128.9,128.9,121.3$, 114.6, 91.4, 63.1, 52.3, 37.8, 30.4, 23.9, $23.1 \mathrm{ppm}$. HRMS (ESI-TOF) $\mathrm{m} / \mathrm{z}:[\mathrm{M}+\mathrm{Na}]^{+}$Calcd for $\mathrm{C}_{26} \mathrm{H}_{28} \mathrm{O}_{6} \mathrm{~S}_{2}=$ 523.1225; Found 523.1223.<smiles>CC(CC=O)CC(CC(Sc1ccccc1)c1ccccc1)(Sc1ccccc1)S(=O)c1ccccc1</smiles>

(S)-3-methyl-5,5-bis(phenylsulfonyl)-7-(phenylthio)heptanal (2t): purified by flash chromatography (on silica, petroleum ether/ ethyl acetate $=6 / 1, \mathrm{v} / \mathrm{v})$, colorless oil, $238 \mathrm{mg}, 92 \%$ yield; $95 \%$ ee; $[\alpha]_{\mathrm{D}}{ }^{24}=+11.6(\mathrm{c}=$ 4.0, $\mathrm{CH}_{3} \mathrm{CN}$ ); The enantiomeric excess was determined by HPLC on Chiralpak AS-3 column, hexane: isopropanol $=85: 15$; flow rate $=1.0 \mathrm{~mL} / \mathrm{min}$; $\mathrm{UV}$ detection at $210 \mathrm{~nm} ; \mathrm{t}_{\mathrm{R}}=36.5 \mathrm{~min}$ (major), $44.9 \mathrm{~min}$ (minor). ${ }^{1} \mathrm{H}$ NMR $\left(400 \mathrm{MHz}, \mathrm{CDCl}_{3}\right)$ 8: $9.68-9.48(\mathrm{~m}, 1 \mathrm{H}), 7.96-7.90(\mathrm{~m}, 4 \mathrm{H}), 7.72-7.66(\mathrm{~m}, 2 \mathrm{H}), 7.58-7.52(\mathrm{~m}$, 4H), $7.39-7.35(\mathrm{~m}, 2 \mathrm{H}), 7.33-7.28(\mathrm{~m}, 2 \mathrm{H}), 7.28-7.23(\mathrm{~m}, 1 \mathrm{H}), 3.23-3.11(\mathrm{~m}, 2 \mathrm{H}), 2.63-2.52(\mathrm{~m}, 4 \mathrm{H})$, $2.32-2.21(\mathrm{~m}, 2 \mathrm{H}), 2.13-2.06(\mathrm{~m}, 1 \mathrm{H}), 0.98(\mathrm{~d}, J=6.8 \mathrm{~Hz}, 3 \mathrm{H}) .{ }^{13} \mathrm{C} \mathrm{NMR}\left(101 \mathrm{MHz}, \mathrm{CDCl}_{3}\right) \delta: 201.1,137.3$, 137.0, 134.8, 134.8, 134.3, 131.2, 131.2, 131.1, 129.2, 128.9, 128.9, 127.2, 91.9, 52.3, 37.9, 31.89, 29.4, 23.8, 23.0 ppm. HRMS (ESI-TOF) $\mathrm{m} / \mathrm{z}:[\mathrm{M}+\mathrm{Na}]^{+}$Calcd for $\mathrm{C}_{26} \mathrm{H}_{28} \mathrm{O}_{5} \mathrm{~S}_{3}=539.0997$; Found 539.0995.

$\mathrm{OHC}$

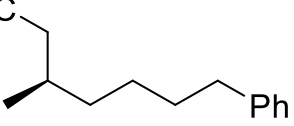

(R)-3-methyl-7-phenylheptanal (2u): purified by flash chromatography (on silica, petroleum ether/ ethyl acetate $=20 / 1, \mathrm{v} / \mathrm{v})$, colorless oil, $68 \mathrm{mg}, 66 \%$ yield; $65 \%$ ee; $[\alpha]_{\mathrm{D}}^{24}=+9.9\left(\mathrm{c}=1.8, \mathrm{CH}_{3} \mathrm{CN}\right)$; The enantiomeric 
excess was determined by HPLC after $\mathrm{NaBH}_{4}$ reduction to the corresponding alcohol on Chiralpak AD-3 column, hexane: isopropanol $=99.5: 0.5$; flow rate $=1.0 \mathrm{~mL} / \mathrm{min} ; \mathrm{UV}$ detection at $210 \mathrm{~nm} ; \mathrm{t}_{\mathrm{R}}=35.9$ (minor), 38.0 min (major). ${ }^{1} \mathrm{H}$ NMR $\left(400 \mathrm{MHz}, \mathrm{CDCl}_{3}\right) \delta: 9.88-9.59(\mathrm{~m}, 1 \mathrm{H}), 7.30-7.24(\mathrm{~m}, 2 \mathrm{H}), 7.19-7.13(\mathrm{~m}, 3 \mathrm{H})$, $2.60(\mathrm{t}, J=7.7 \mathrm{~Hz}, 2 \mathrm{H}), 2.41-2.34(\mathrm{~m}, 1 \mathrm{H}), 2.24-2.17(\mathrm{~m}, 1 \mathrm{H}), 2.08-2.00(\mathrm{~m}, 1 \mathrm{H}), 1.63-1.57(\mathrm{~m}, 2 \mathrm{H})$, $1.41-1.27(\mathrm{~m}, 4 \mathrm{H}), 0.94(\mathrm{~d}, J=6.7 \mathrm{~Hz}, 3 \mathrm{H}) .{ }^{13} \mathrm{C} \mathrm{NMR}\left(101 \mathrm{MHz}, \mathrm{CDCl}_{3}\right) \delta: 203.1,142.6,128.4,128.3,125.7$, 51.1, 36.7, 35.9, 31.5, 28.1, 26.6, 20.0 ppm. HRMS (ESI-TOF) $\mathrm{m} / \mathrm{z}:[\mathrm{M}+\mathrm{Na}]^{+}$Calcd for $\mathrm{C}_{14} \mathrm{H}_{20} \mathrm{O}=205.1592$; Found 205.1587. 


\section{Procedures for synthetic transformations ${ }^{4}$}

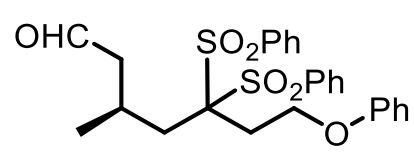

2s

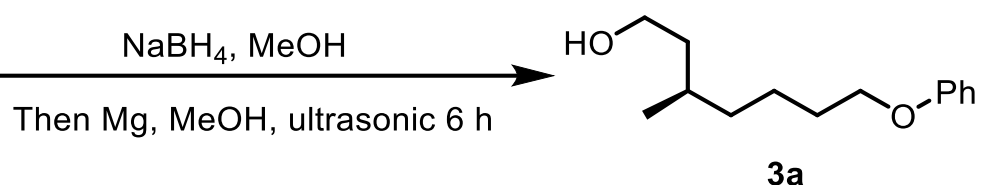

$3 a$

In a vial, (S)-3-methyl-7-phenoxy-5,5-bis(phenylsulfonyl)heptanal (2s) (50 mg, $0.1 \mathrm{mmol})$ was added in 1 $\mathrm{mL}$ of methanol at $0{ }^{\circ} \mathrm{C}$. Next, sodium borohydride $(5.7 \mathrm{mg}, 0.15 \mathrm{mmol})$ was added and the reaction was stirred for half an hour. The reaction mixture was poured to a separation flask containing $\mathrm{HCl}(37 \%)$ and ethyl acetate. The organic layer was dried over $\mathrm{MgSO}_{4}$ and the solvent was removed under reduced pressure. The residue was transferred to a tube $(10 \mathrm{ml})$ and dry methanol $(5 \mathrm{~mL})$ and activated magnesium turnings $(60 \mathrm{mg}, 2.5 \mathrm{mmol})$ were added. Then the mixture was ultrasound for 6 hours. The reaction mixture was quenched with $3 \mathrm{M} \mathrm{HCl}$ at $0{ }^{\circ} \mathrm{C}$. The aqueous layer was extracted with diethyl ether. The combined organic layers were washed with saturated aqueous $\mathrm{NaHCO}_{3}$ solution and brine, dried over $\mathrm{MgSO}_{4}$, and concentrated in vacuo. The residue was purified by flash chromatography (on silica, petroleum ether/ ethyl acetate $=10 / 1, \mathrm{v} / \mathrm{v}$ ) to give the desired product.

(R)-3-methyl-7-phenoxyheptan-1-ol (3a): colorless oil, $20 \mathrm{mg}, 88 \%$ yield; $96 \%$ ee; $[\alpha]_{\mathrm{D}}{ }^{24}=+5.8(\mathrm{c}=0.5$, $\mathrm{CH}_{3} \mathrm{CN}$ ); The enantiomeric excess was determined by HPLC on Chiralpak AS-H column, hexane: isopropanol $=99.5: 0.5$; flow rate $=1.0 \mathrm{~mL} / \mathrm{min} ; \mathrm{UV}$ detection at $230 \mathrm{~nm} ; \mathrm{t}_{\mathrm{R}}=39.116 \mathrm{~min}$ (major), $43.879 \mathrm{~min}$ (minor). ${ }^{1} \mathrm{H}$ NMR $\left(600 \mathrm{MHz}, \mathrm{CDCl}_{3}\right) \delta: 7.31-7.24(\mathrm{~m}, 2 \mathrm{H}), 6.96-6.85(\mathrm{~m}, 3 \mathrm{H}), 3.95(\mathrm{t}, J=6.5 \mathrm{~Hz}, 2 \mathrm{H}), 3.73-3.63(\mathrm{~m}$, 2H), $1.81-1.72(\mathrm{~m}, 2 \mathrm{H}), 1.65-1.57(\mathrm{~m}, 2 \mathrm{H}), 1.54-1.36(\mathrm{~m}, 4 \mathrm{H}), 1.25-1.18(\mathrm{~m}, 1 \mathrm{H}), 0.91(\mathrm{~d}, J=6.5 \mathrm{~Hz}$, 3H). ${ }^{13} \mathrm{C} \mathrm{NMR}\left(151 \mathrm{MHz}, \mathrm{CDCl}_{3}\right) \delta: 159.1,129.4,120.5,114.5,67.8,61.2,39.9,36.8,29.5,29.5,23.5,19.6$ ppm. HRMS (ESI-TOF) m/z : [M+H] $]^{+}$Calcd for $\mathrm{C}_{14} \mathrm{H}_{22} \mathrm{O}_{2}=223.1698$; Found 223.1691.

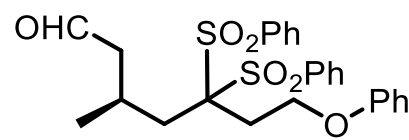

2s

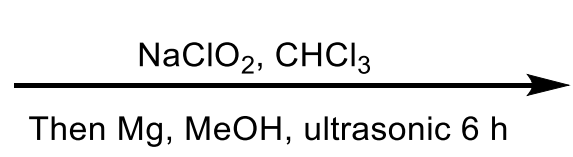

Then $\mathrm{Mg}, \mathrm{MeOH}$, ultrasonic $6 \mathrm{~h}$

To a vial containing aldehyde $2 \mathrm{~s}\left(50 \mathrm{mg}, 1\right.$ equiv.) dissolved in $\mathrm{CHCl}_{3}(1 \mathrm{~mL})$, isopentane $(25.2 \mathrm{mg}, 3.6$ equiv.), tert-butanol (12.6 mg, 1.7 equiv.), water (7.9 mg, 4.4 equiv.), $\mathrm{NaH}_{2} \mathrm{PO}_{4}$ (19.2 mg, 1.6 equiv.) and $\mathrm{NaClO}_{2}(12 \mathrm{mg}, 1.6$ equiv.) were sequentially added. The reaction was stirred overnight. The reaction mixture was quenched with water and extracted into EtOAc ( 3 times). The combined organic layer was dried over $\mathrm{MgSO}_{4}$ and the solvent was removed under reduced pressure. The residue was transferred to a tube (10 $\left.\mathrm{ml}\right)$ and 
dry methanol $(5 \mathrm{~mL})$ and activated magnesium turnings $(60 \mathrm{mg}, 2.5 \mathrm{mmol})$ were added. Then the mixture was ultrasound for 6 hours. The reaction mixture was quenched with $3 \mathrm{M} \mathrm{HCl}$ at $0{ }^{\circ} \mathrm{C}$. The aqueous layer was extracted with diethyl ether. The combined organic layers were washed with brine( 3 times), dried over $\mathrm{MgSO}_{4}$, and concentrated in vacuo. The residue was purified by flash chromatography (on silica, petroleum ether/ ethyl acetate $=10 / 1, \mathrm{v} / \mathrm{v})$ to give the desired product.

(R)-3-methyl-7-phenoxyheptanoic acid (3b): colorless oil, $19 \mathrm{mg}, 79 \%$ yield; $98 \%$ ee; $[\alpha]_{\mathrm{D}}{ }^{24}=+5.2(\mathrm{c}=0.5$, $\mathrm{CH}_{3} \mathrm{CN}$ ); The enantiomeric excess was determined by HPLC on Chiralpak AS-H column, hexane: isopropanol $=90: 10 ;$ flow rate $=0.8 \mathrm{~mL} / \mathrm{min} ; \mathrm{UV}$ detection at $220 \mathrm{~nm} ; \mathrm{t}_{\mathrm{R}}=9.3 \mathrm{~min}$ (major), $8.7 \mathrm{~min}$ (minor). ${ }^{1} \mathrm{H} \mathrm{NMR}$ $\left(400 \mathrm{MHz}, \mathrm{CDCl}_{3}\right) \delta: 7.30-7.25(\mathrm{~m}, 2 \mathrm{H}), 6.96-6.86(\mathrm{~m}, 3 \mathrm{H}), 3.96(\mathrm{t}, J=6.4 \mathrm{~Hz}, 2 \mathrm{H}), 2.40-2.33(\mathrm{~m}, 1 \mathrm{H})$, $2.21-2.14(\mathrm{~m}, 1 \mathrm{H}), 2.04-1.97(\mathrm{~m}, 1 \mathrm{H}), 1.83-1.71(\mathrm{~m}, 2 \mathrm{H}), 1.56-1.40(\mathrm{~m}, 3 \mathrm{H}), 1.32-1.28(\mathrm{~m}, 1 \mathrm{H}), 0.99$ $(\mathrm{d}, J=6.6 \mathrm{~Hz}, 3 \mathrm{H}) .{ }^{13} \mathrm{C} \mathrm{NMR}\left(101 \mathrm{MHz}, \mathrm{CDCl}_{3}\right) \delta: 178.7,159.1,129.4,120.5,114.5,67.7,41.4,36.3,30.1$, 29.4, 23.5, 19.6 ppm. HRMS (ESI-TOF) $\mathrm{m} / \mathrm{z}:[\mathrm{M}-\mathrm{H}]^{-}$Calcd for $\mathrm{C}_{14} \mathrm{H}_{22} \mathrm{O}_{3}=235.1334$; Found 235.1334.

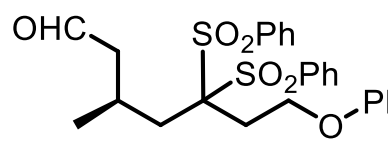

2s

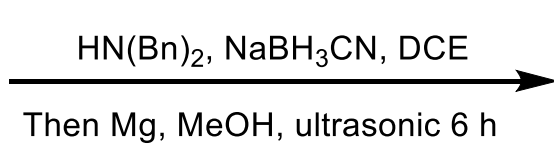

Then $\mathrm{Mg}, \mathrm{MeOH}$, ultrasonic $6 \mathrm{~h}$

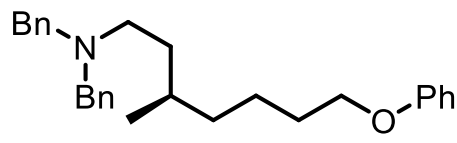

3c

Dibenzylamine (32 mg, $0.16 \mathrm{mmol}, 1$ equiv.) and aldehyde $2 \mathrm{~s}$ ( $80 \mathrm{mg}, 0.16 \mathrm{mmol}, 1$ equiv.) were mixed in 1,2-dichloroethane ( $1 \mathrm{~mL}$ ) and treated with sodium cyanoborohydride (14 mg, $0.22 \mathrm{mmol}, 1.4$ equiv.). The mixture was stirred at room temperature under an argon atmosphere until the reactants were consumed. The reaction mixture was quenched by adding aq. $1 \mathrm{M} \mathrm{NaOH}$ and was extracted with diethyl ether. The combined organic layer was washed with brine and dried over $\mathrm{MgSO}_{4}$. The solvent was evaporated. The residue was transferred to a tube $(25 \mathrm{ml})$ and dry methanol $(8 \mathrm{~mL})$ and activated magnesium turnings $(96 \mathrm{mg}, 4 \mathrm{mmol})$ were added. Then the mixture was ultrasound for 6 hours. The reaction mixture was quenched with $3 \mathrm{M} \mathrm{HCl}$ at $0{ }^{\circ} \mathrm{C}$ and saturated aqueous $\mathrm{NaHCO}_{3}$ was added until the gas had ceased. The aqueous layer was extracted with diethyl ether. The combined organic layers were washed with brine, dried over $\mathrm{MgSO}_{4}$, and concentrated in vacuo. The residue was purified by flash chromatography (on silica, petroleum ether/ ethyl acetate $=20 / 1, \mathrm{v} / \mathrm{v}$ ) to give the desired product.

(R)- $N, N$-dibenzyl-3-methyl-7-phenoxyheptan-1-amine (3c): colorless oil, $53 \mathrm{mg}, 82 \%$ yield; $96 \%$ ee; $[\alpha]_{\mathrm{D}}{ }^{24}$ $=+2.7\left(\mathrm{c}=2.0, \mathrm{CH}_{3} \mathrm{CN}\right)$; The enantiomeric excess was determined by HPLC on Chiralpak OD-H column, 
hexane: isopropanol $=85: 15$; flow rate $=1.0 \mathrm{~mL} / \mathrm{min}$; $\mathrm{UV}$ detection at $210 \mathrm{~nm} ; \mathrm{t}_{\mathrm{R}}=7.2 \mathrm{~min}$ (major), $16.1 \mathrm{~min}$ (minor). ${ }^{1} \mathrm{H}$ NMR $\left(600 \mathrm{MHz}, \mathrm{CDCl}_{3}\right) \delta: 7.35(\mathrm{~d}, J=7.4 \mathrm{~Hz}, 4 \mathrm{H}), 7.32-7.24(\mathrm{~m}, 6 \mathrm{H}), 7.22-7.19(\mathrm{~m}, 2 \mathrm{H}), 6.92$ (t, $J=7.3 \mathrm{~Hz}, 1 \mathrm{H}), 6.88(\mathrm{~d}, J=8.1 \mathrm{~Hz}, 2 \mathrm{H}), 3.90(\mathrm{t}, J=6.6 \mathrm{~Hz}, 2 \mathrm{H}), 3.53(\mathrm{q}, J=13.6 \mathrm{~Hz}, 4 \mathrm{H}), 2.46-2.38$ (m, $2 \mathrm{H}), 1.73-1.64(\mathrm{~m}, 2 \mathrm{H}), 1.56-1.46(\mathrm{~m}, 2 \mathrm{H}), 1.45-1.39(\mathrm{~m}, 1 \mathrm{H}), 1.36-1.29(\mathrm{~m}, 2 \mathrm{H}), 1.25-1.20(\mathrm{~m}, 1 \mathrm{H})$, $1.12-1.05(\mathrm{~m}, 1 \mathrm{H}), 0.76(\mathrm{~d}, J=6.5 \mathrm{~Hz}, 3 \mathrm{H}) .{ }^{13} \mathrm{C} \mathrm{NMR}\left(151 \mathrm{MHz}, \mathrm{CDCl}_{3}\right)$ ): 159.2, 140.1, 129.4, 128.8, 128.17, 126.8, 120.5, 114.6, 67.9, 58.4, 51.3, 36.7, 34.1, 30.8, 29.6, 23.5, 19.8 ppm. HRMS (ESI-TOF) m/z : [M+H] ${ }^{+}$ Calcd for $\mathrm{C}_{28} \mathrm{H}_{35} \mathrm{NO}=402.2797$; Found 402.2789. 


\section{NMR spectra}<smiles></smiles>

${ }^{1} \mathrm{H}$ NMR (400 MHz, $\left.\mathrm{CDCl}_{3}\right)$

$\underbrace{3}$

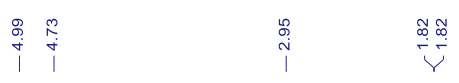

$\mathcal{L} \sum_{\mathrm{SO}_{2} \mathrm{Ph}}^{\mathrm{Me}}$

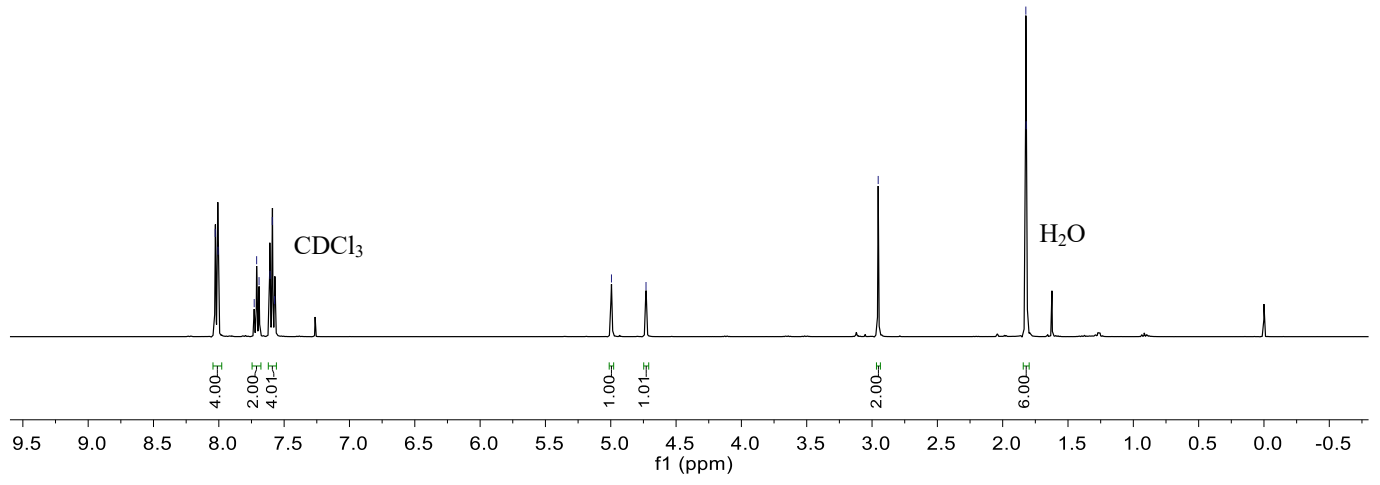

${ }^{13} \mathrm{C}$ NMR (101 MHz, CDCl 3$)$

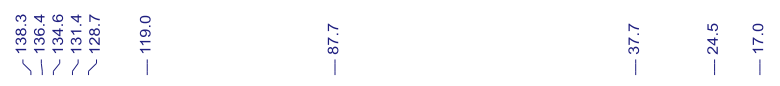

$1 . \mathrm{Me}_{\mathrm{SO}_{2} \mathrm{Ph}}$

$\mathrm{SO}_{2} \mathrm{Ph}$

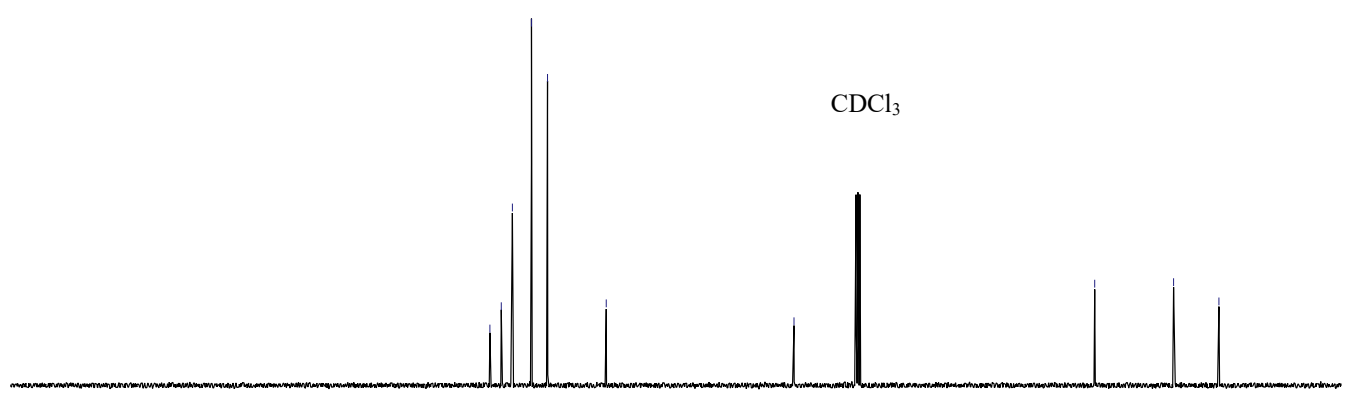

$\begin{array}{llllllllllllllllllllll}210 & 200 & 190 & 180 & 170 & 160 & 150 & 140 & 130 & 120 & 110 & 100 & 90 & 80 & 70 & 60 & 50 & 40 & 30 & 20 & 10 & 0\end{array}$ 
$\overbrace{}^{\mathrm{Et}} \mathrm{SO}_{\mathrm{SO}_{2} \mathrm{Ph}} \mathrm{Ph}$

${ }^{1} \mathrm{H}$ NMR (400 MHz, $\left.\mathrm{CDCl}_{3}\right)$

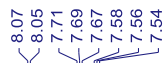

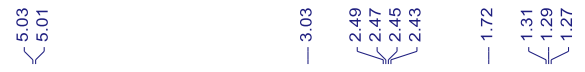

$\mu \underbrace{\mathrm{Et}}_{\mathrm{SO}_{2} \mathrm{Ph}} \mathrm{SO}_{2} \mathrm{Ph}$

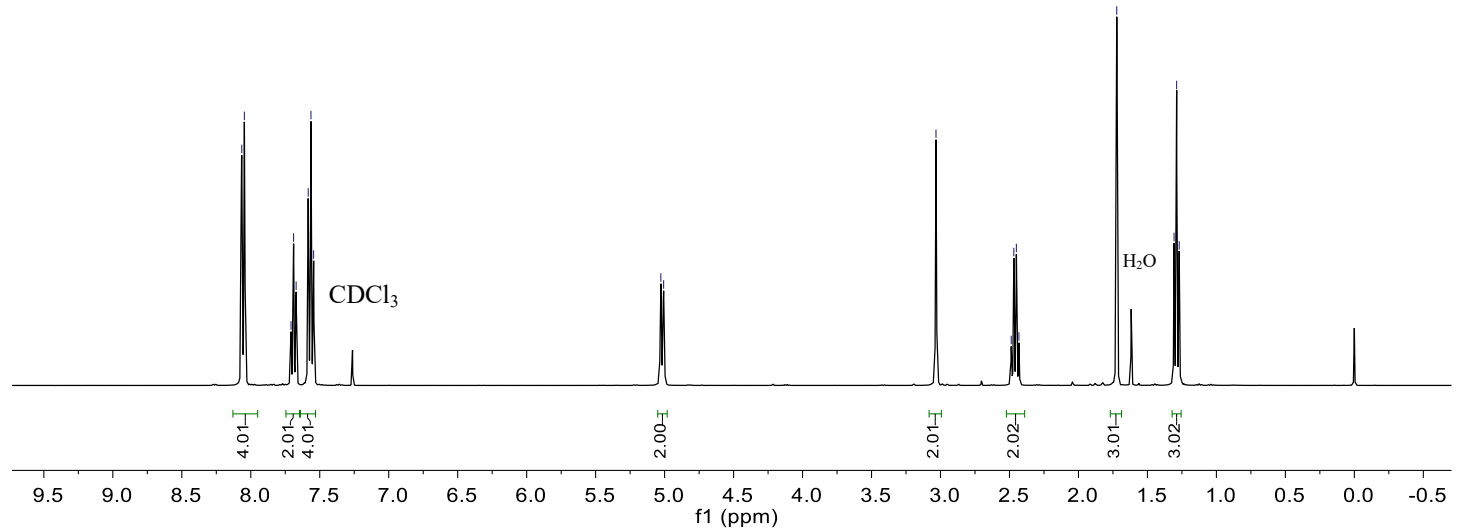

${ }^{13} \mathrm{C}$ NMR (101 MHz, $\left.\mathrm{CDCl}_{3}\right)$

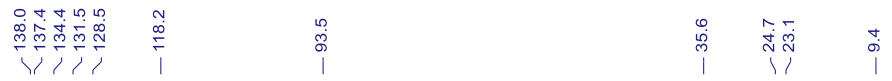

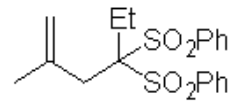

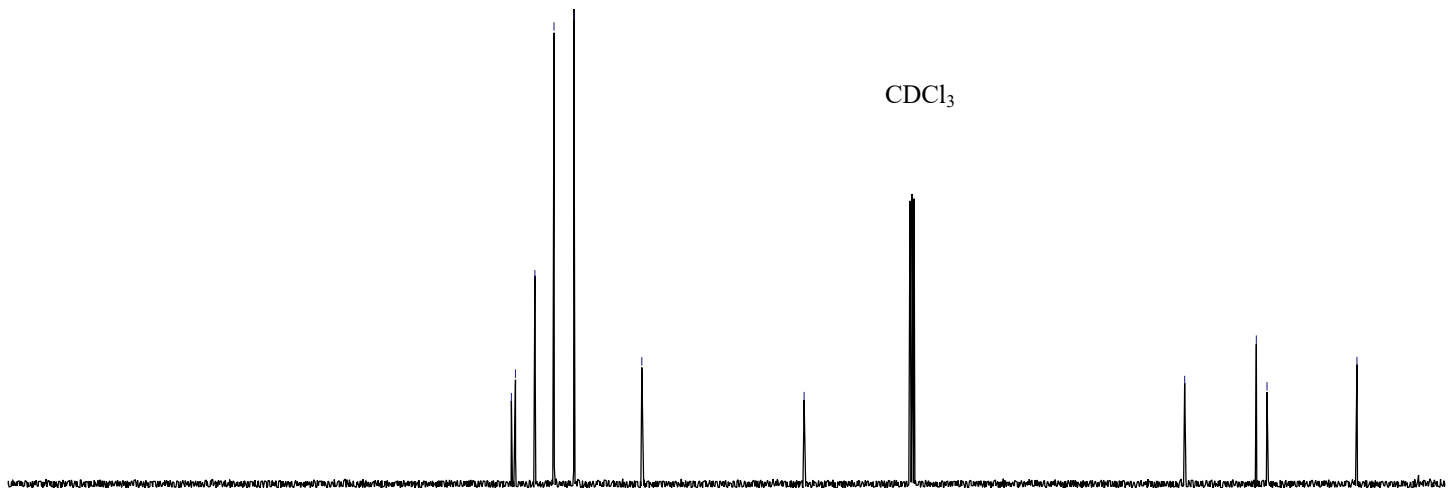

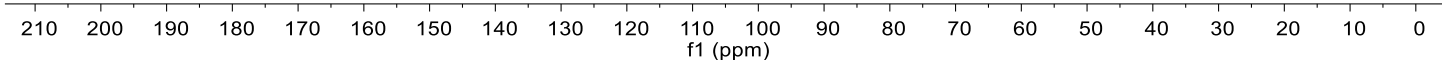


$\underbrace{\stackrel{n-\mathrm{Pr}}{\mathrm{SO}_{2} \mathrm{Ph}}} \mathrm{SO}_{2} \mathrm{Ph} 1 \mathrm{c}$

${ }^{1} \mathrm{H}$ NMR (400 MHz, $\left.\mathrm{CDCl}_{3}\right)$

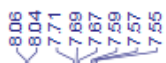

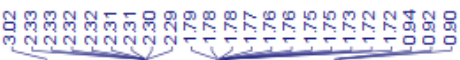

$\underbrace{n-\mathrm{Pr}_{\mathrm{SO}_{2} \mathrm{Ph}}}_{\mathrm{SO}_{2} \mathrm{Ph}}$

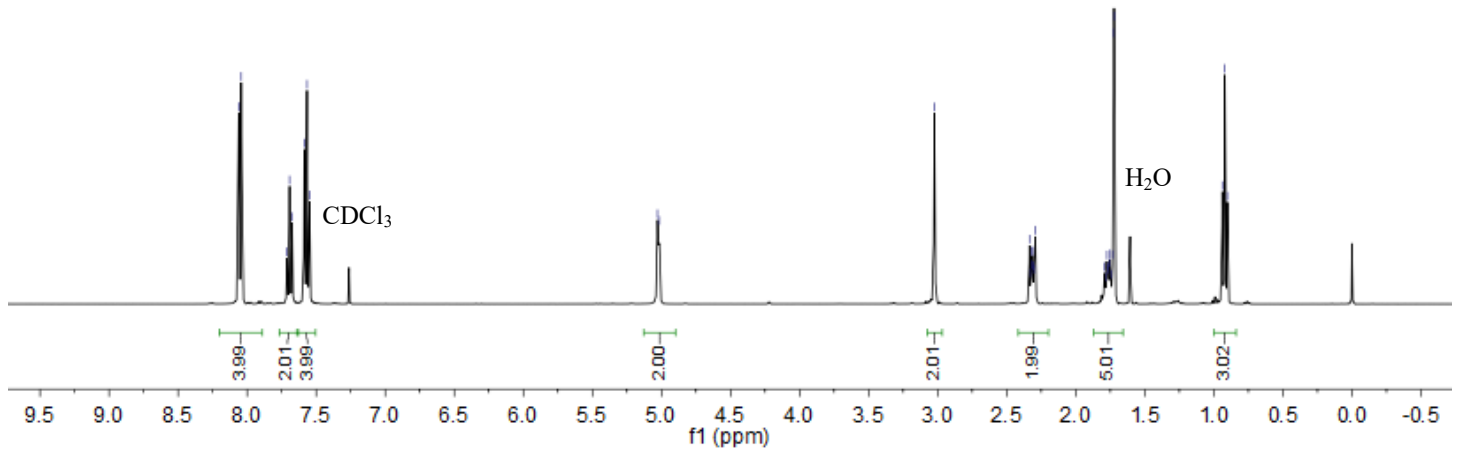

${ }^{13} \mathrm{C}$ NMR (101 MHz, $\left.\mathrm{CDCl}_{3}\right)$

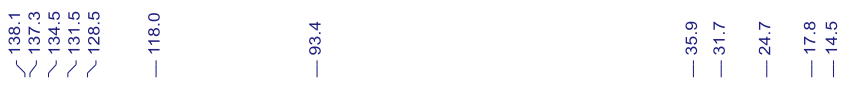

$\| \sum_{\mathrm{SO}_{2} \mathrm{Ph}}^{\mathrm{n} \mathrm{Pr}}$

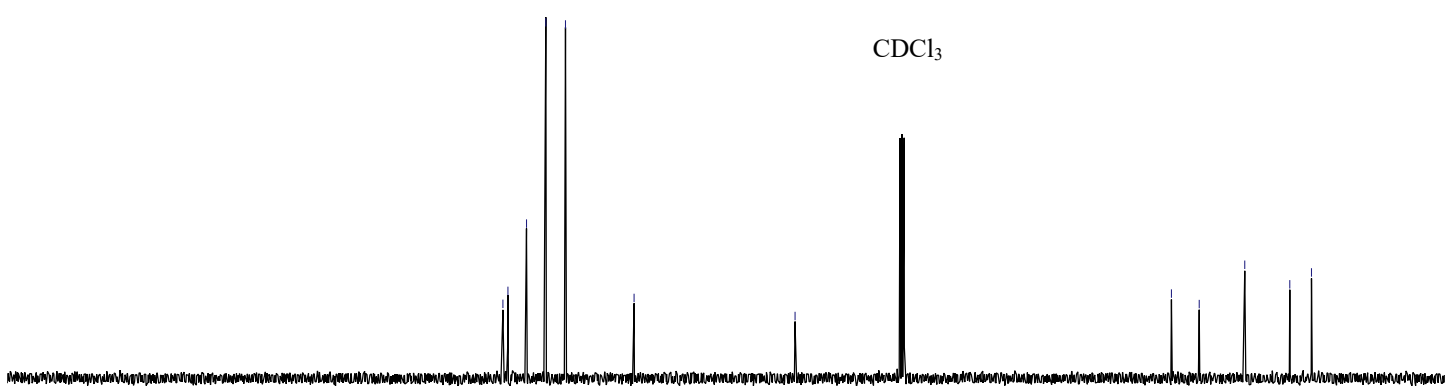

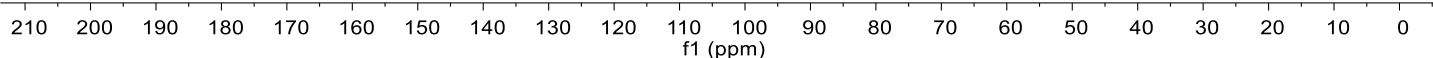




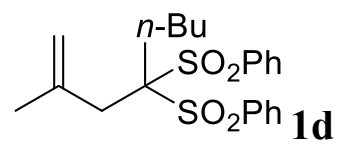

${ }^{1} \mathrm{H}$ NMR (400 MHz, $\left.\mathrm{CDCl}_{3}\right)$

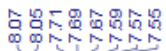

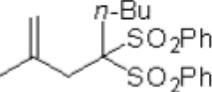

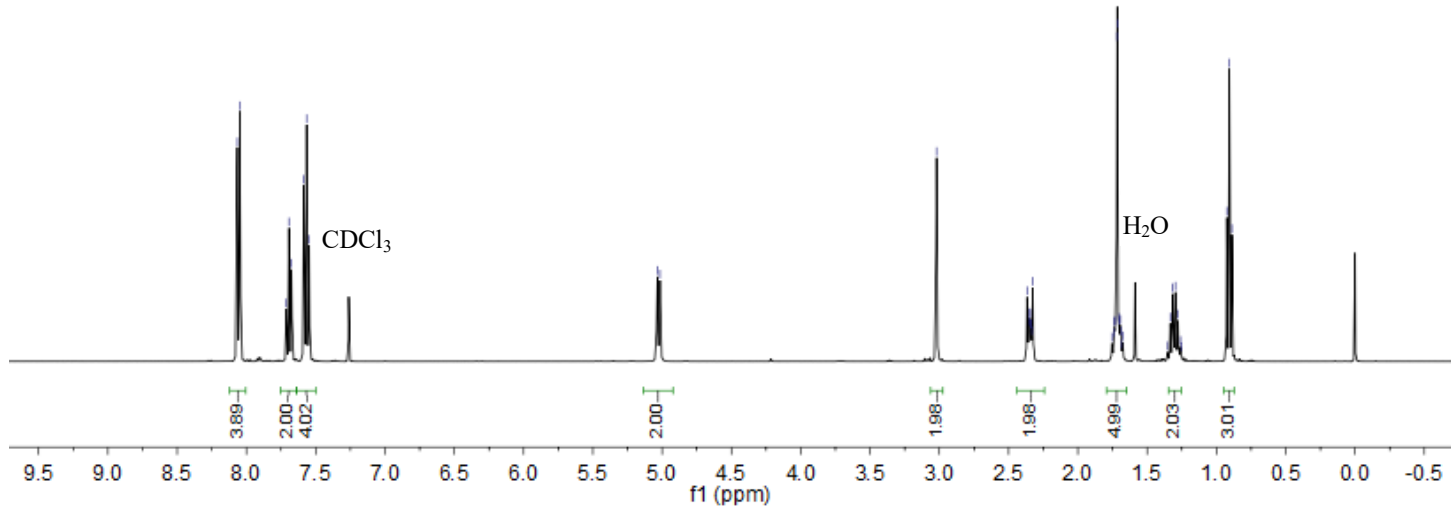

${ }^{13} \mathrm{C}$ NMR (101 MHz, $\left.\mathrm{CDCl}_{3}\right)$

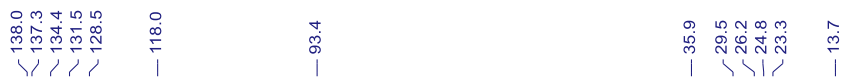

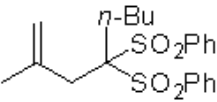

$\mathrm{CDCl}_{3}$

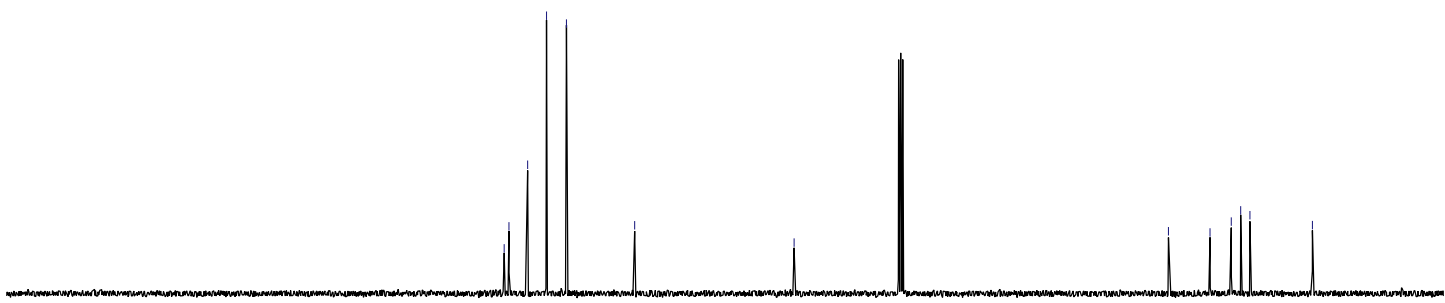

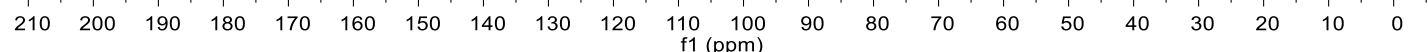


$\underbrace{-i-\mathrm{Bu}} \mathrm{SO}_{\mathrm{SO}_{2} \mathrm{Ph}} \mathrm{Ph}$

${ }^{1} \mathrm{H}$ NMR (400 MHz, $\left.\mathrm{CDCl}_{3}\right)$

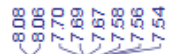

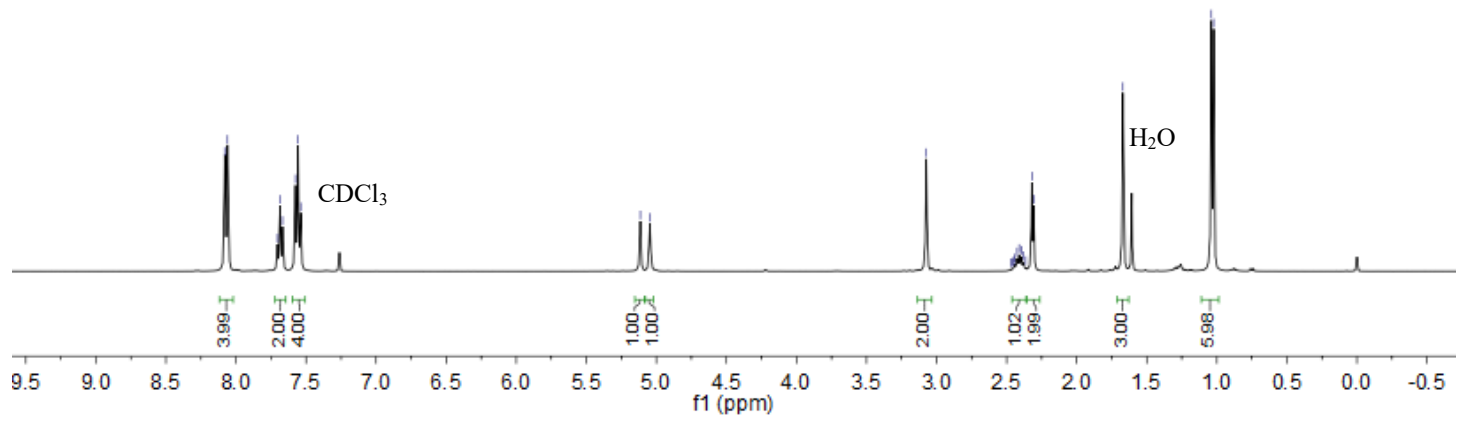

${ }^{13} \mathrm{C}$ NMR (101 MHz, $\left.\mathrm{CDCl}_{3}\right)$<smiles>C=C(C)CC([Se])([Se]O[Se])[Se]Oc1ccccc1</smiles>

$\mathrm{CDCl}_{3}$

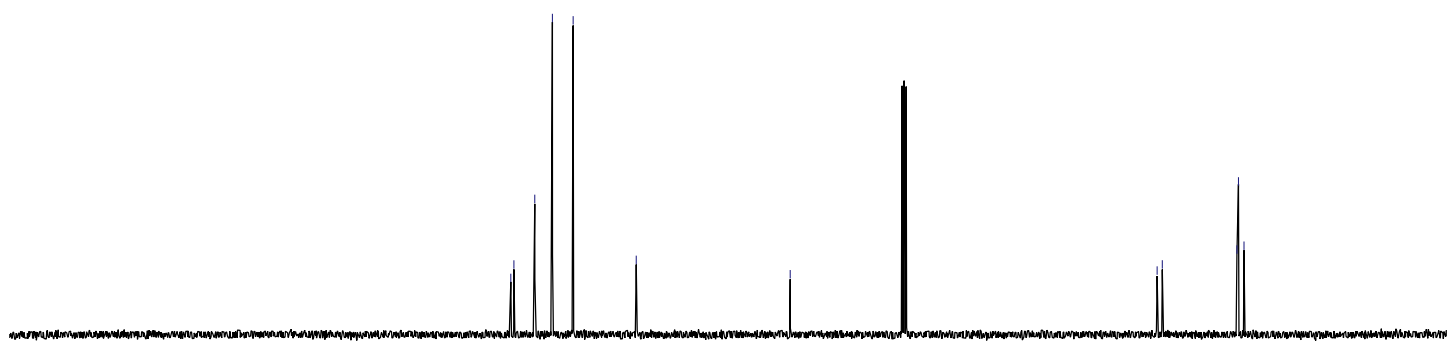

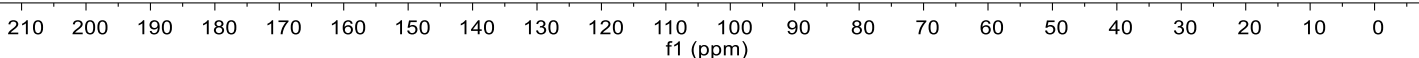




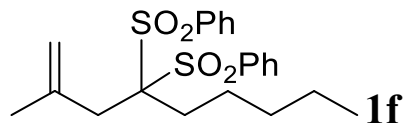

${ }^{1} \mathrm{H}$ NMR (400 MHz, $\left.\mathrm{CDCl}_{3}\right)$

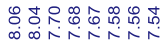
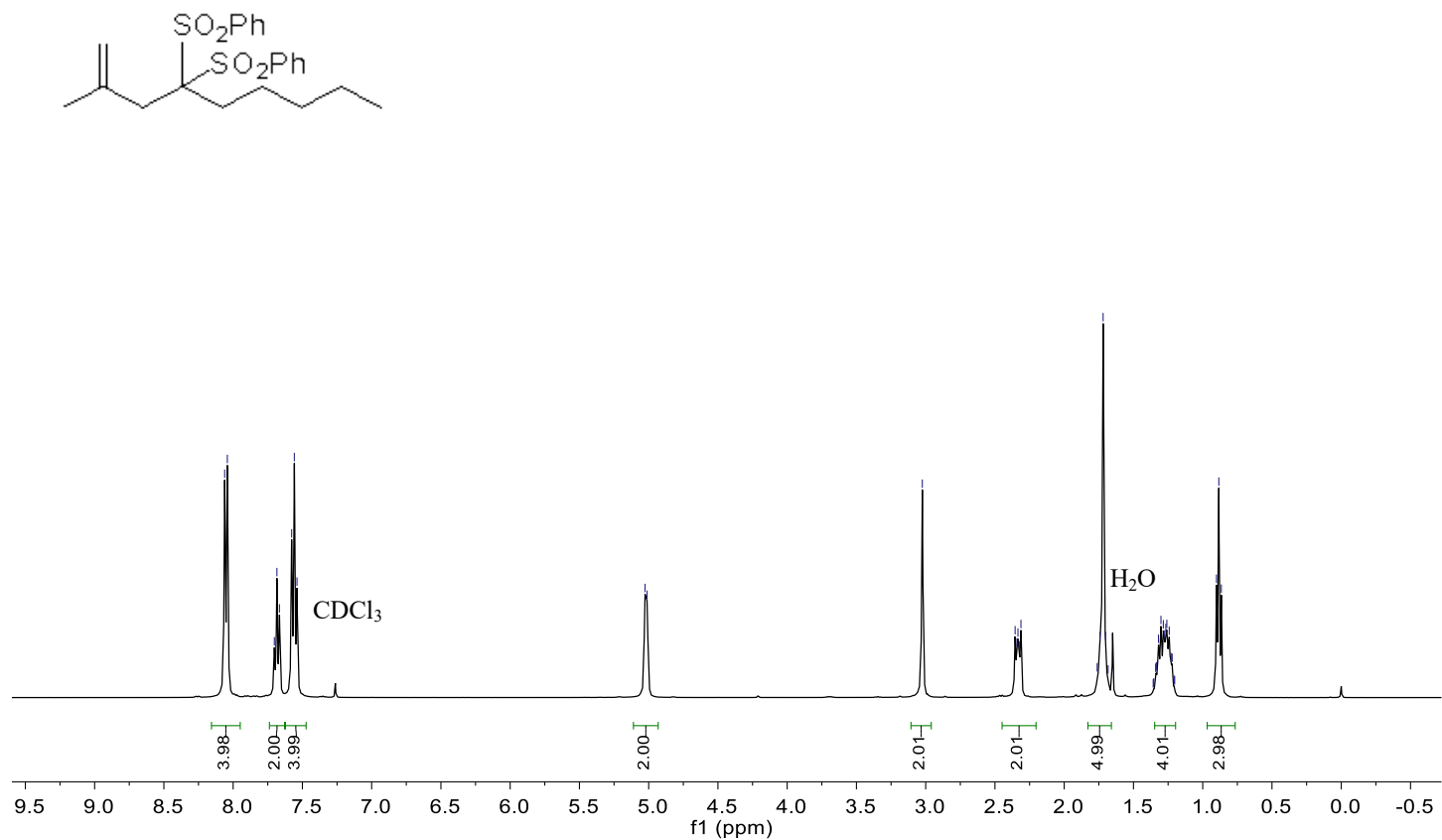

${ }^{13} \mathrm{C}$ NMR (101 MHz, $\left.\mathrm{CDCl}_{3}\right)$

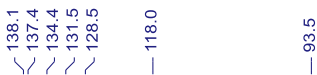
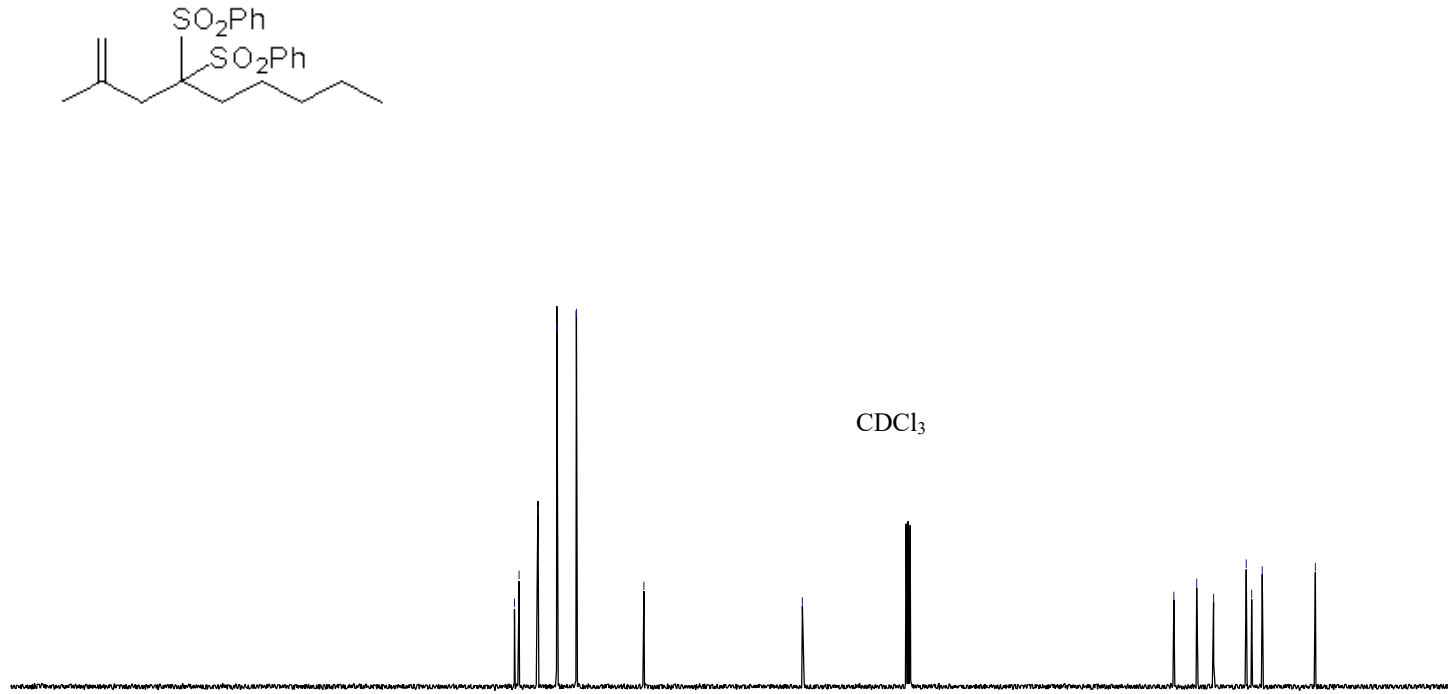

$\begin{array}{lllllllllllllllllllll}210 & 200 & 190 & 180 & 170 & 160 & 150 & 140 & 130 & 120 & \begin{array}{c}110 \\ \mathrm{f} 1(\mathrm{ppm})\end{array} & 100 & 80 & 70 & 60 & 50 & 40 & 30 & 20 & 10 & 0\end{array}$ 


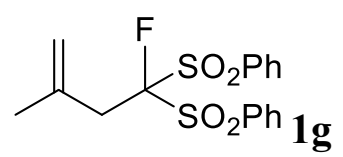

${ }^{1} \mathrm{H}$ NMR (400 MHz, $\left.\mathrm{CDCl}_{3}\right)$

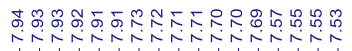<smiles>C=C(C)CC(F)(F)[Se](F)(Oc1ccccc1)Oc1ccccc1</smiles>

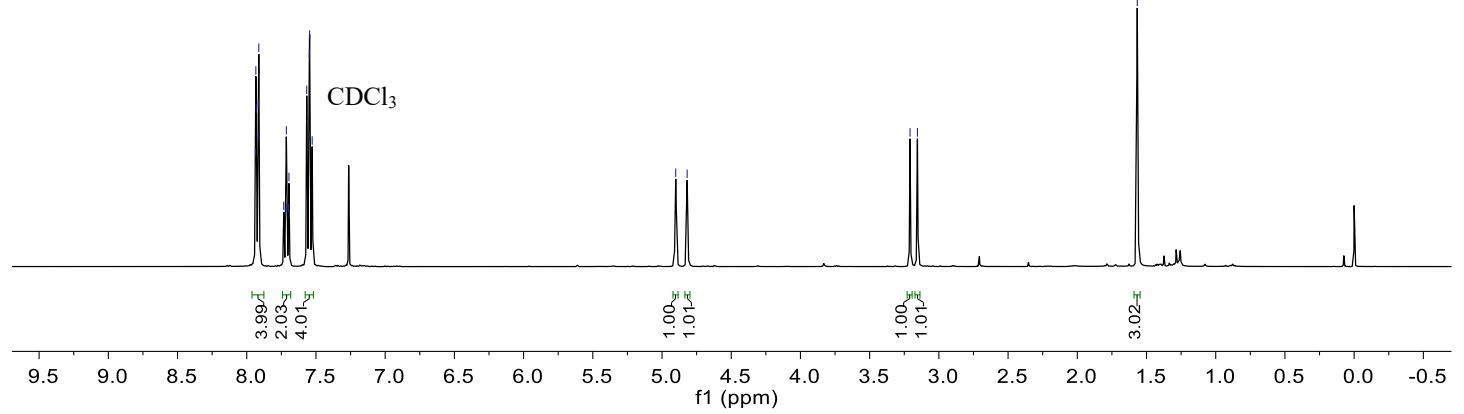

${ }^{13} \mathrm{C}$ NMR (101 MHz, $\left.\mathrm{CDCl}_{3}\right)$<smiles>C=C(C)CC(F)(CC(=C)C)O[Po](O)(O)c1ccccc1</smiles>

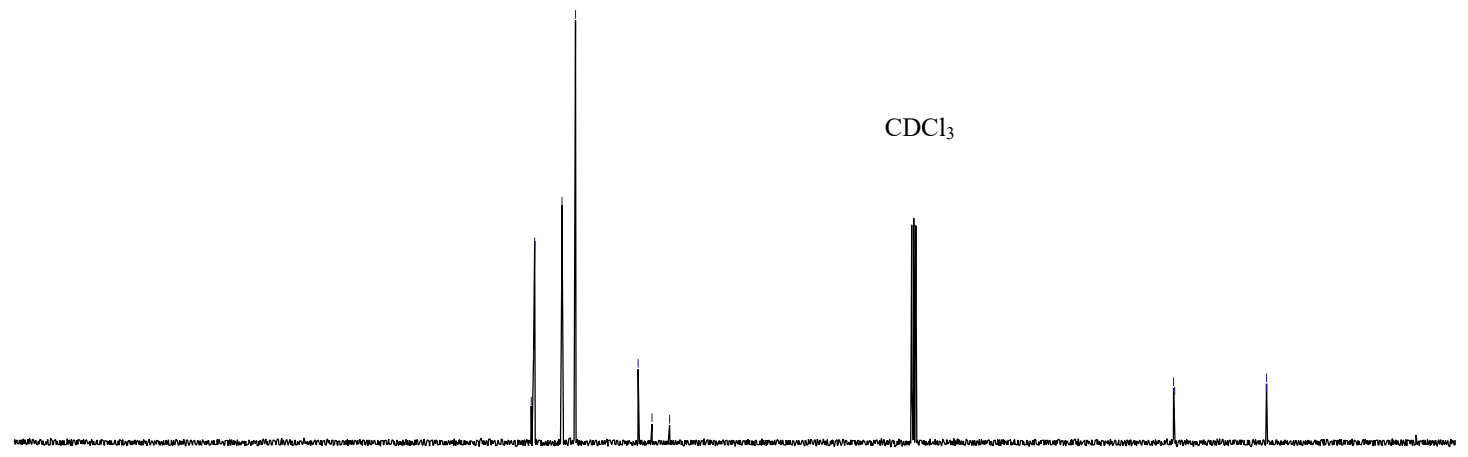

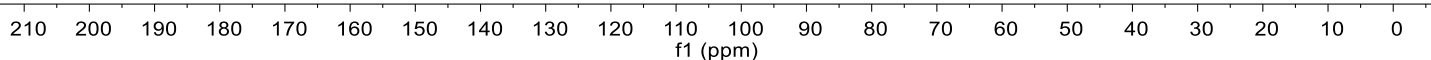


<smiles>C=C(C)CC([Se])(CC(=O)Oc1ccccc1)c1ccccc1</smiles>

${ }^{1} \mathrm{H}$ NMR (400 MHz, $\left.\mathrm{CDCl}_{3}\right)$

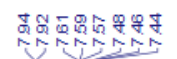

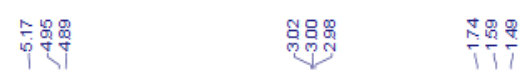
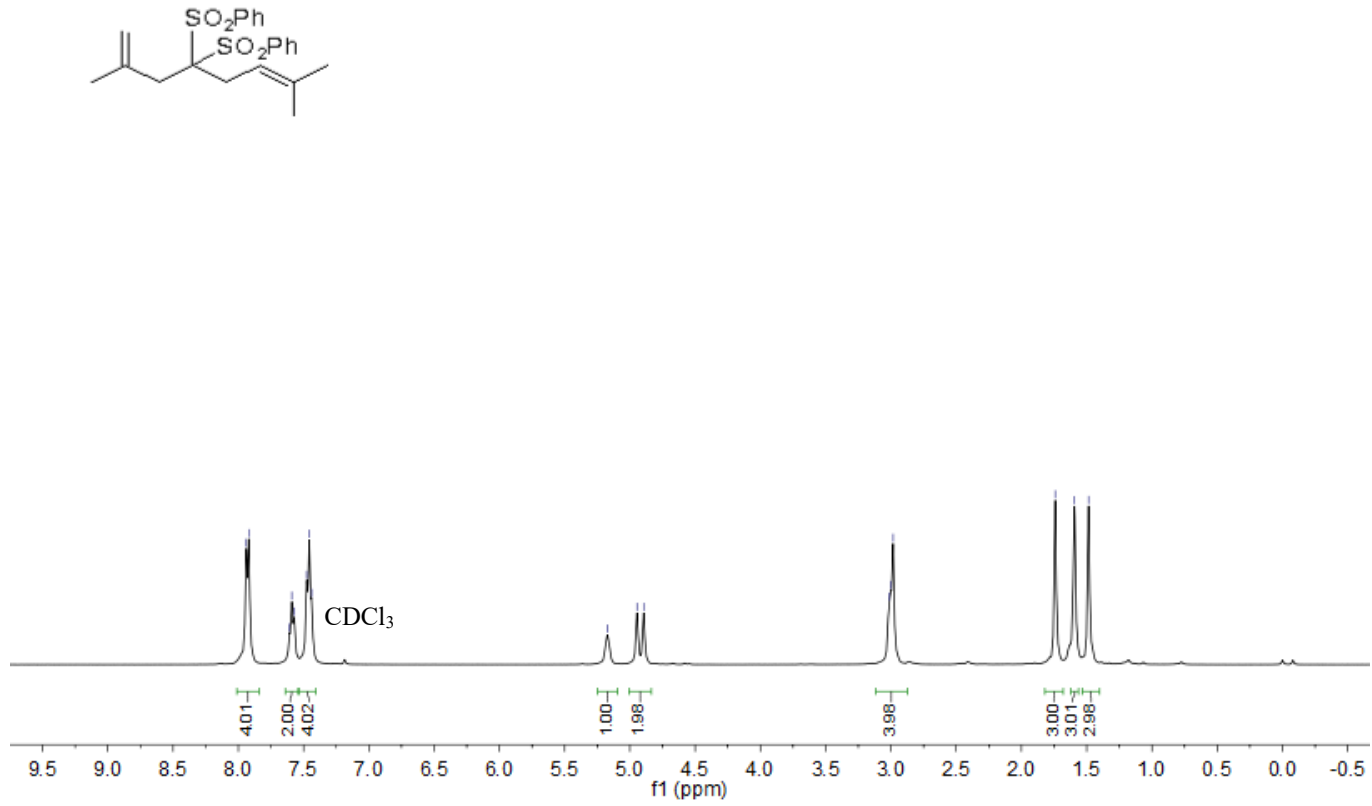

${ }^{13} \mathrm{C}$ NMR (101 MHz, $\left.\mathrm{CDCl}_{3}\right)$
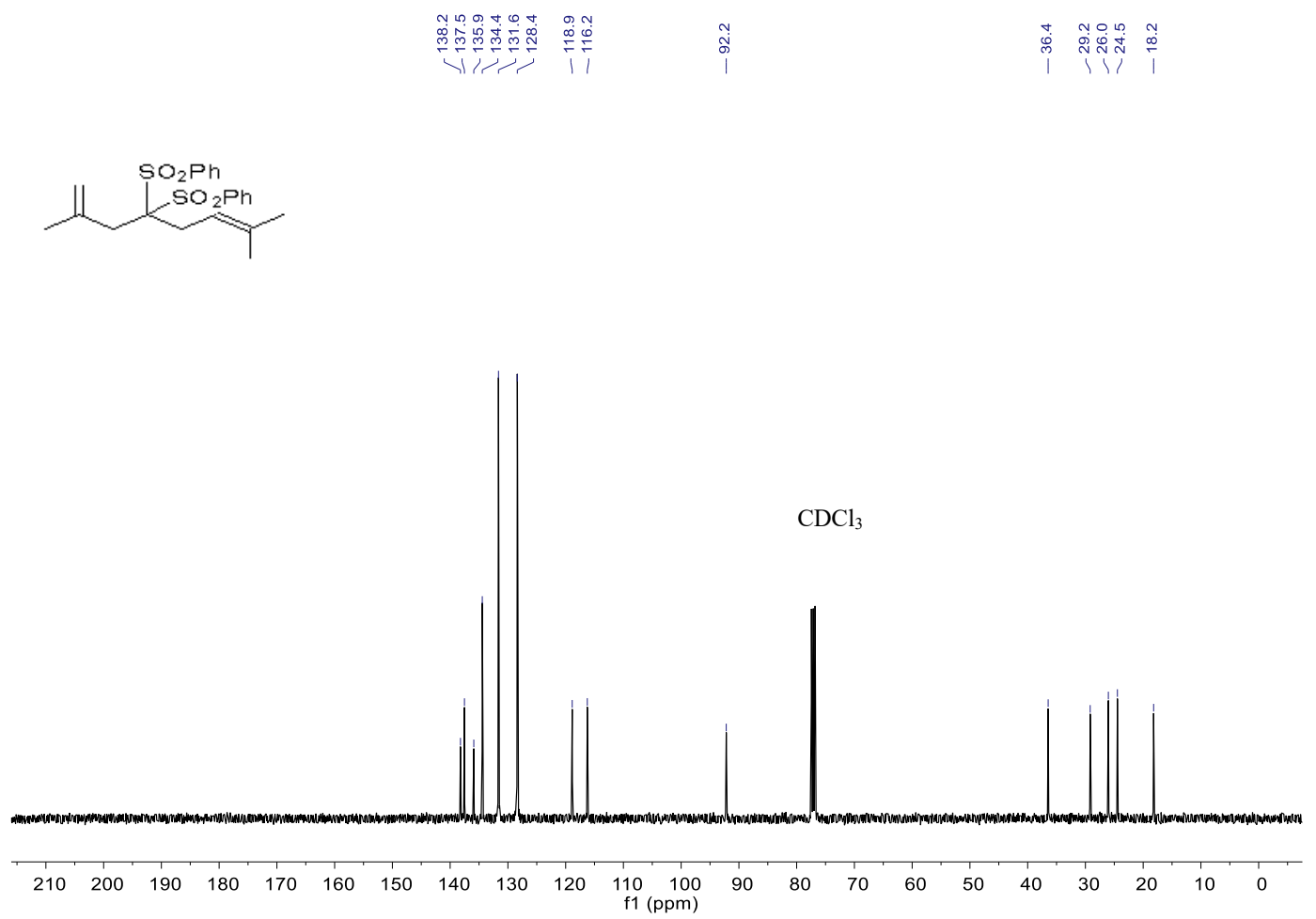


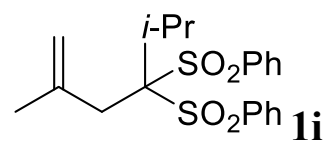

${ }^{1} \mathrm{H}$ NMR (400 MHz, $\left.\mathrm{CDCl}_{3}\right)$

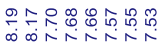

$1 \underbrace{\mathrm{Pr}}_{\mathrm{SO}_{2} \mathrm{Ph}}$

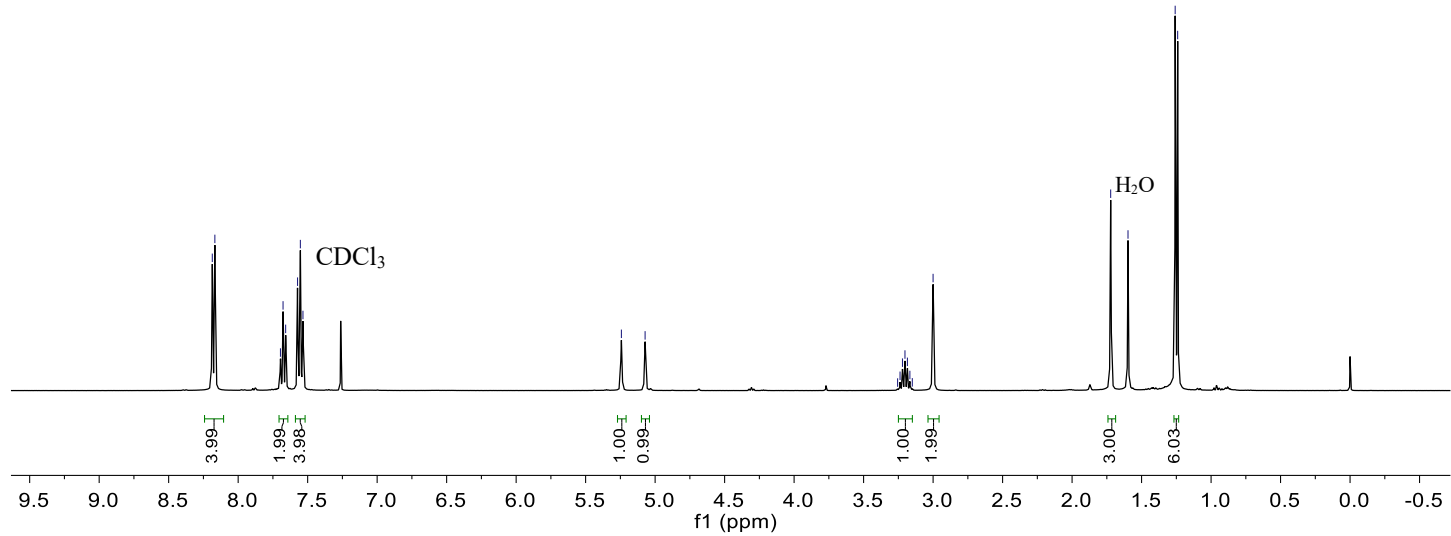

${ }^{13} \mathrm{C}$ NMR (101 MHz, $\left.\mathrm{CDCl}_{3}\right)$

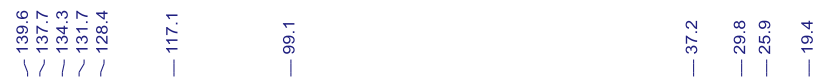

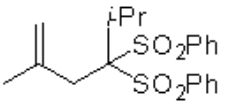

$\mathrm{CDCl}_{3}$

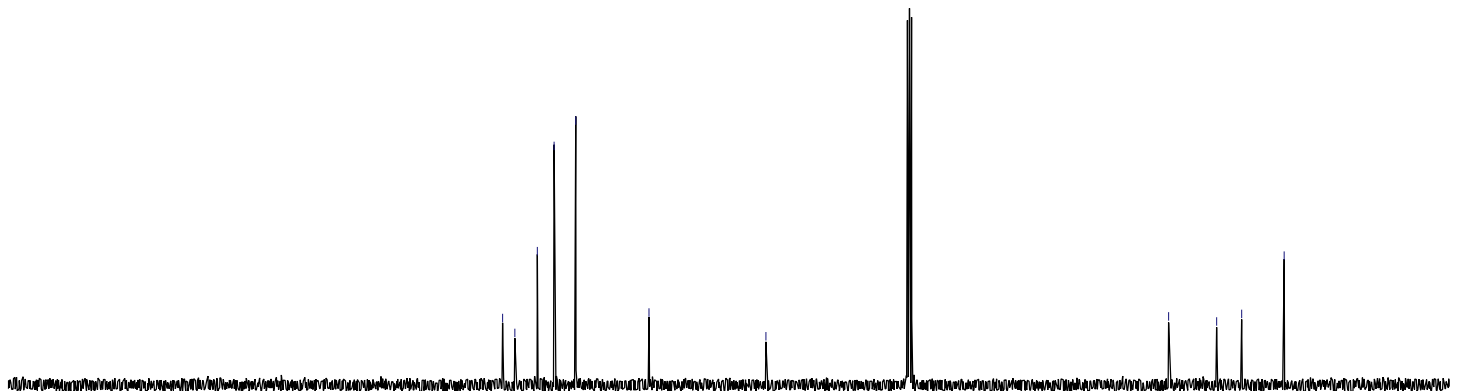

$\begin{array}{lllllllllllllllllllll}210 & 200 & 190 & 180 & 170 & 160 & 150 & 140 & 130 & 120 & \begin{array}{c}110 \\ \mathrm{f} 1(\mathrm{ppm})\end{array} & 90 & 80 & 70 & 60 & 50 & 40 & 30 & 20 & 10 & 0\end{array}$ 


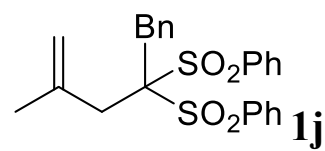

${ }^{1} \mathrm{H}$ NMR (400 MHz, $\left.\mathrm{CDCl}_{3}\right)$

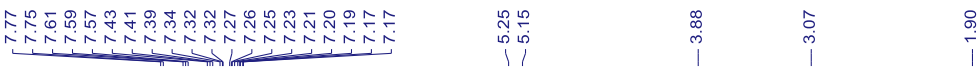<smiles>C=C(C)CC(Br)(Br)C(Br)(Oc1ccccc1)Oc1ccccc1</smiles>

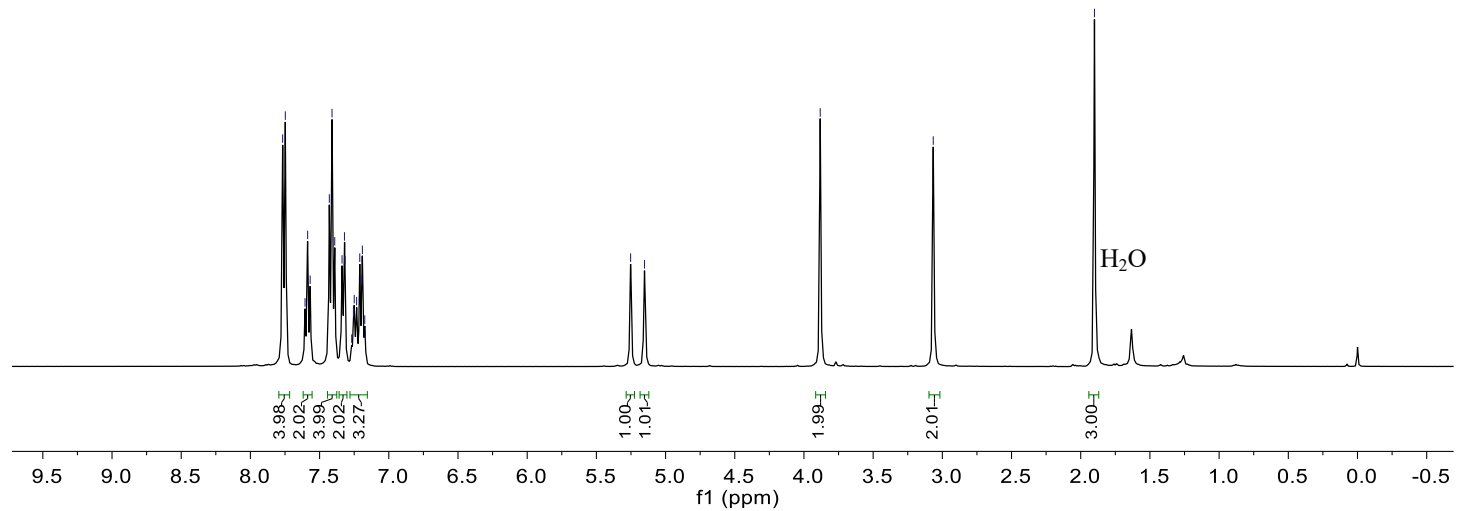

${ }^{13} \mathrm{C}$ NMR (101 MHz, $\left.\mathrm{CDCl}_{3}\right)$

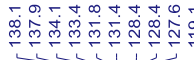

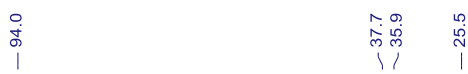

$1 \sum_{\mathrm{SO}_{2} \mathrm{Ph}}^{\mathrm{Bn}}$

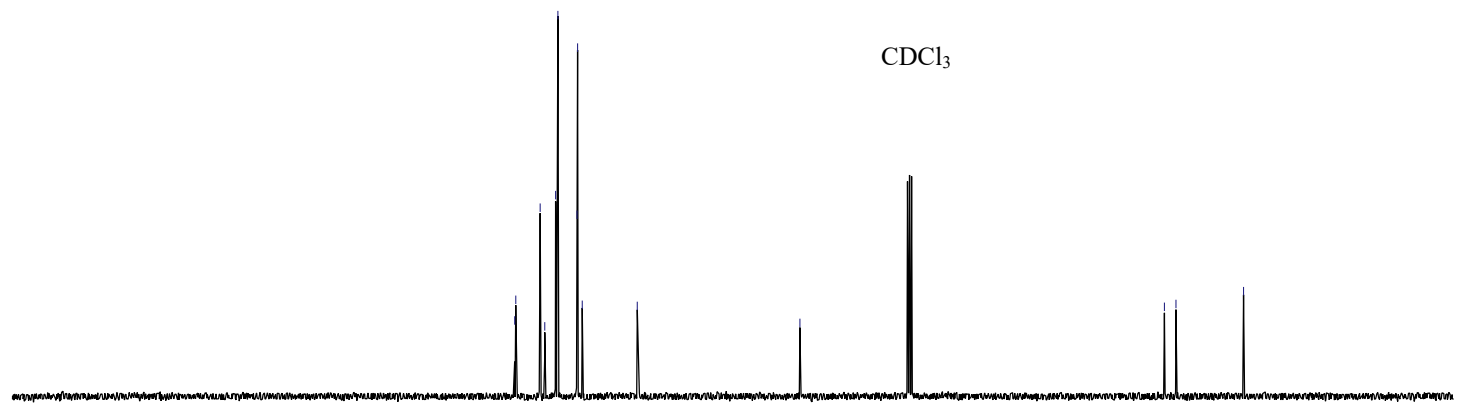

$\begin{array}{llllllllllllllllllllll}210 & 200 & 190 & 180 & 170 & 160 & 150 & 140 & 130 & 120 & 110 & 100 & 90 & 80 & 70 & 60 & 50 & 40 & 30 & 20 & 10 & 0\end{array}$ 


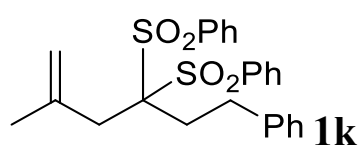

${ }^{1}$ H NMR (400 MHz, $\left.\mathrm{CDCl}_{3}\right)$

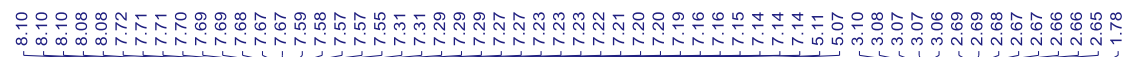
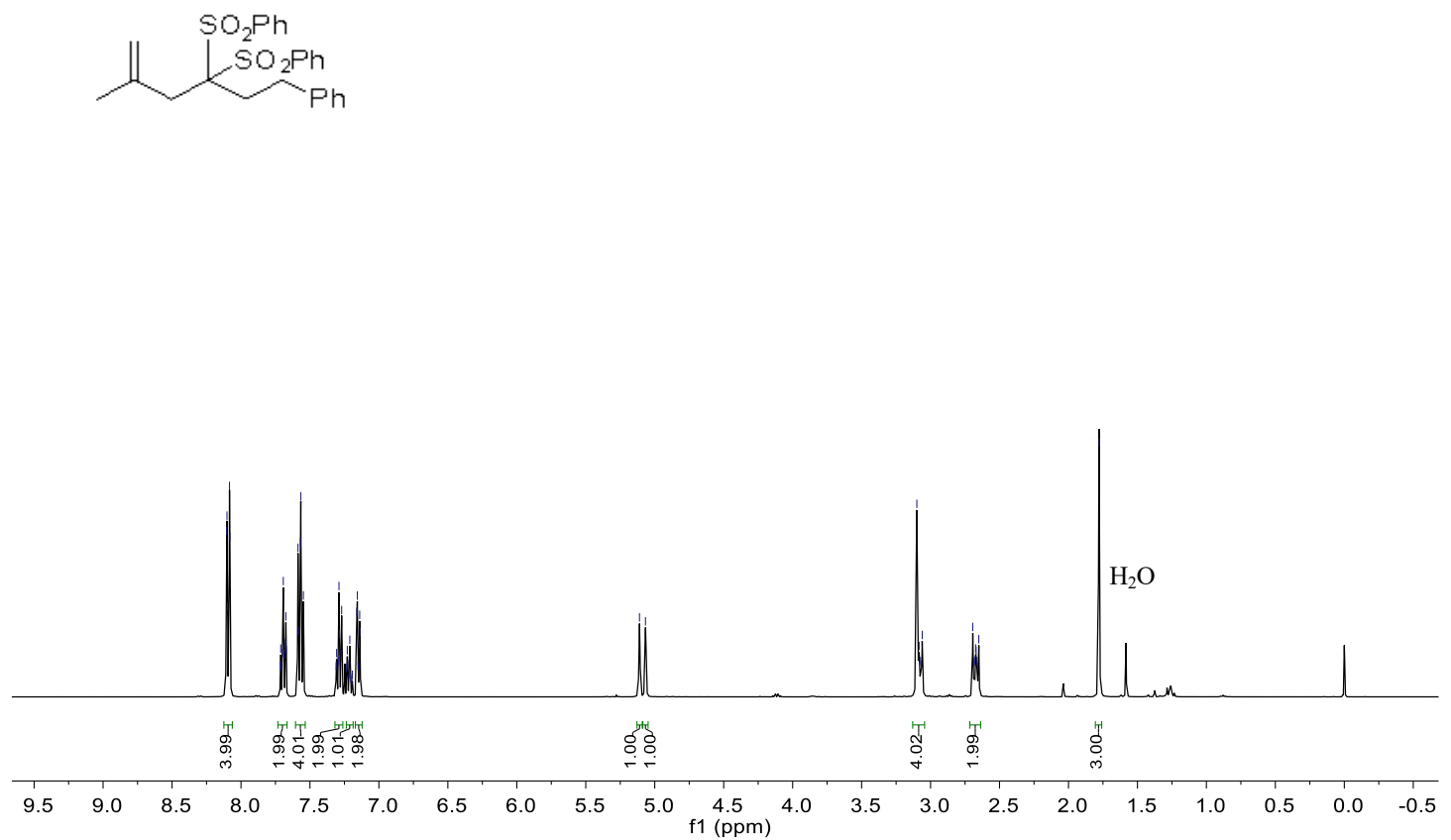

${ }^{13} \mathrm{C}$ NMR (101 MHz, $\left.\mathrm{CDCl}_{3}\right)$

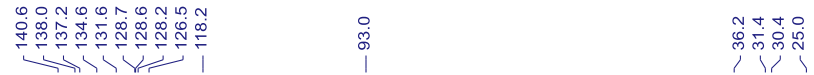

$\underbrace{\mathrm{SO}_{2} \mathrm{Ph}}_{\mathrm{Ph}}$

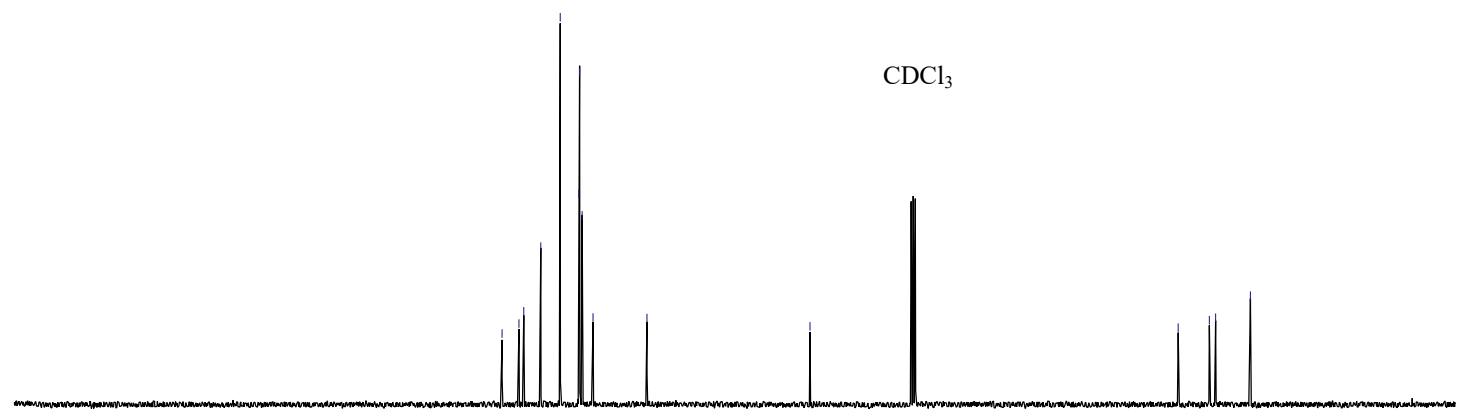

$\begin{array}{lllllllllllllllllllll}210 & 200 & 190 & 180 & 170 & 160 & 150 & 140 & 130 & 120 & \begin{array}{c}110 \\ \mathrm{f} 1(\mathrm{ppm})\end{array} & 90 & 80 & 70 & 60 & 50 & 40 & 30 & 20 & 10 & 0\end{array}$ 
$\underbrace{\mathrm{SO}_{2} \mathrm{Ph}} \mathrm{Ph}_{\mathbf{1 1}}^{\mathrm{SO}_{2} \mathrm{Ph}}$

${ }^{1} \mathrm{H}$ NMR (400 MHz, $\left.\mathrm{CDCl}_{3}\right)$

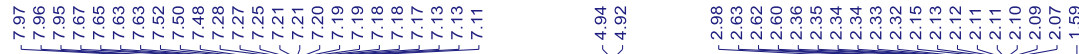

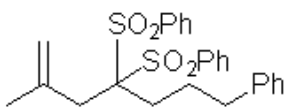

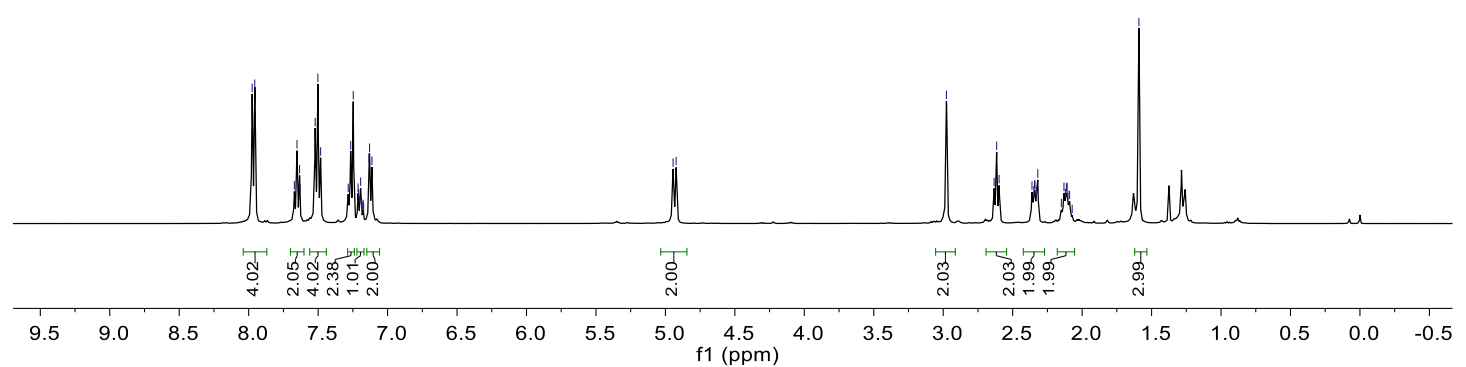

${ }^{13} \mathrm{C}$ NMR (101 MHz, CDCl 3$)$

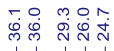

Y<smiles>C=C(C)CC([O-])([Se])CC([Se])(Cc1ccccc1)c1ccccc1</smiles>

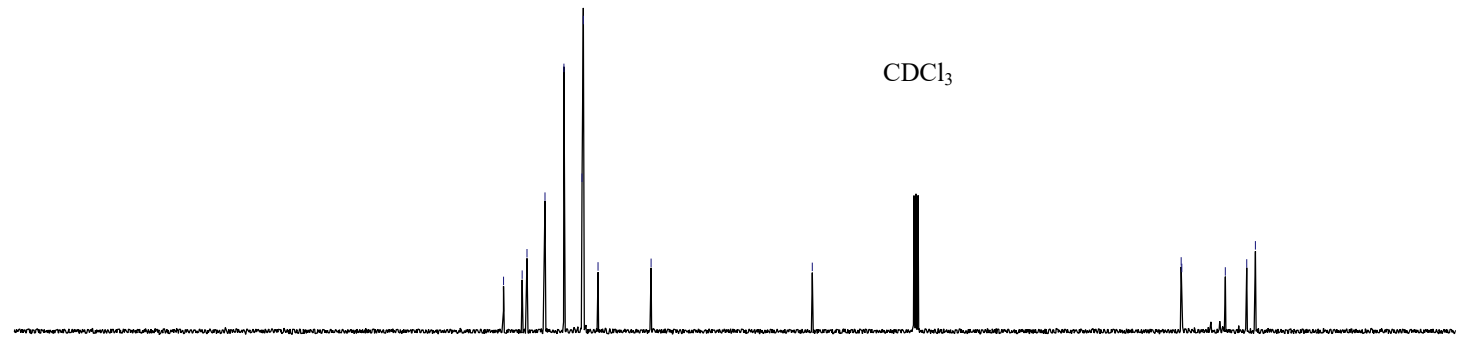

$\begin{array}{lllllllllllllllllllll}210 & 200 & 190 & 180 & 170 & 160 & 150 & 140 & 130 & 120 & \begin{array}{c}110 \\ \mathrm{f} 1(\mathrm{ppm})\end{array} & 100 & 80 & 70 & 60 & 50 & 40 & 30 & 20 & 10 & 0\end{array}$ 


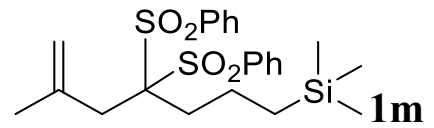

${ }^{1} \mathrm{H}$ NMR (400 MHz, $\left.\mathrm{CDCl}_{3}\right)$

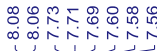
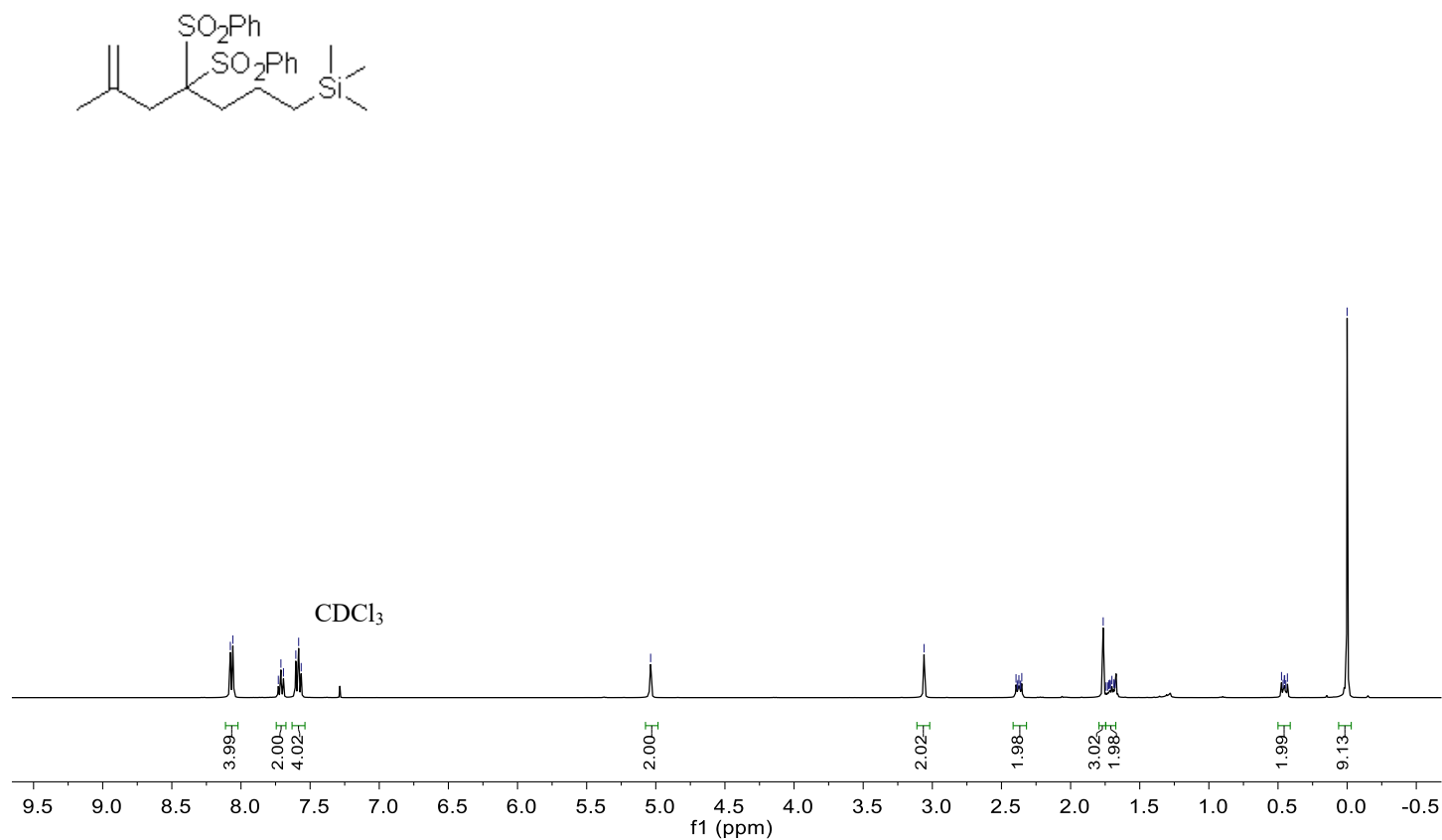

${ }^{13} \mathrm{C}$ NMR (101 MHz, $\left.\mathrm{CDCl}_{3}\right)$

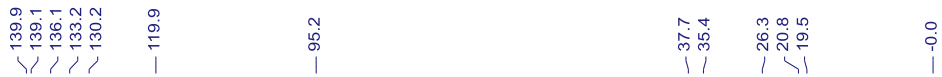

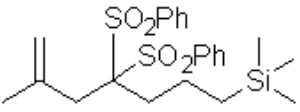

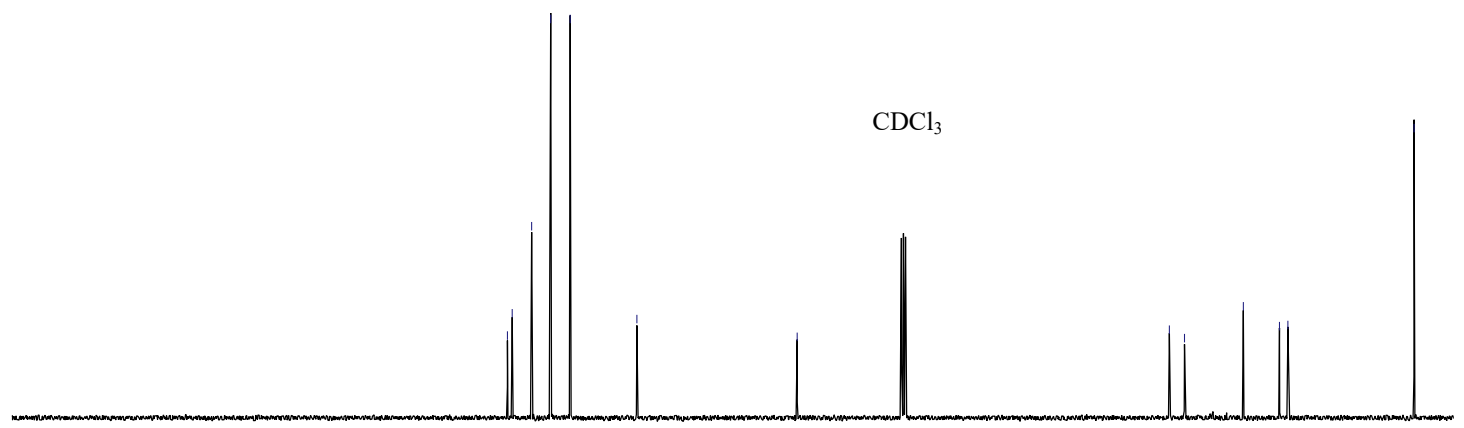

$\begin{array}{lllllllllllllllllllll}210 & 200 & 190 & 180 & 170 & 160 & 150 & 140 & 130 & 120 & \begin{array}{c}110 \\ \mathrm{f} 1(\mathrm{ppm})\end{array} & 100 & 80 & 70 & 60 & 50 & 40 & 30 & 20 & 10 & 0\end{array}$ 


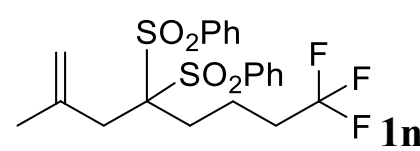

${ }^{1} \mathrm{H}$ NMR (400 MHz, $\left.\mathrm{CDCl}_{3}\right)$
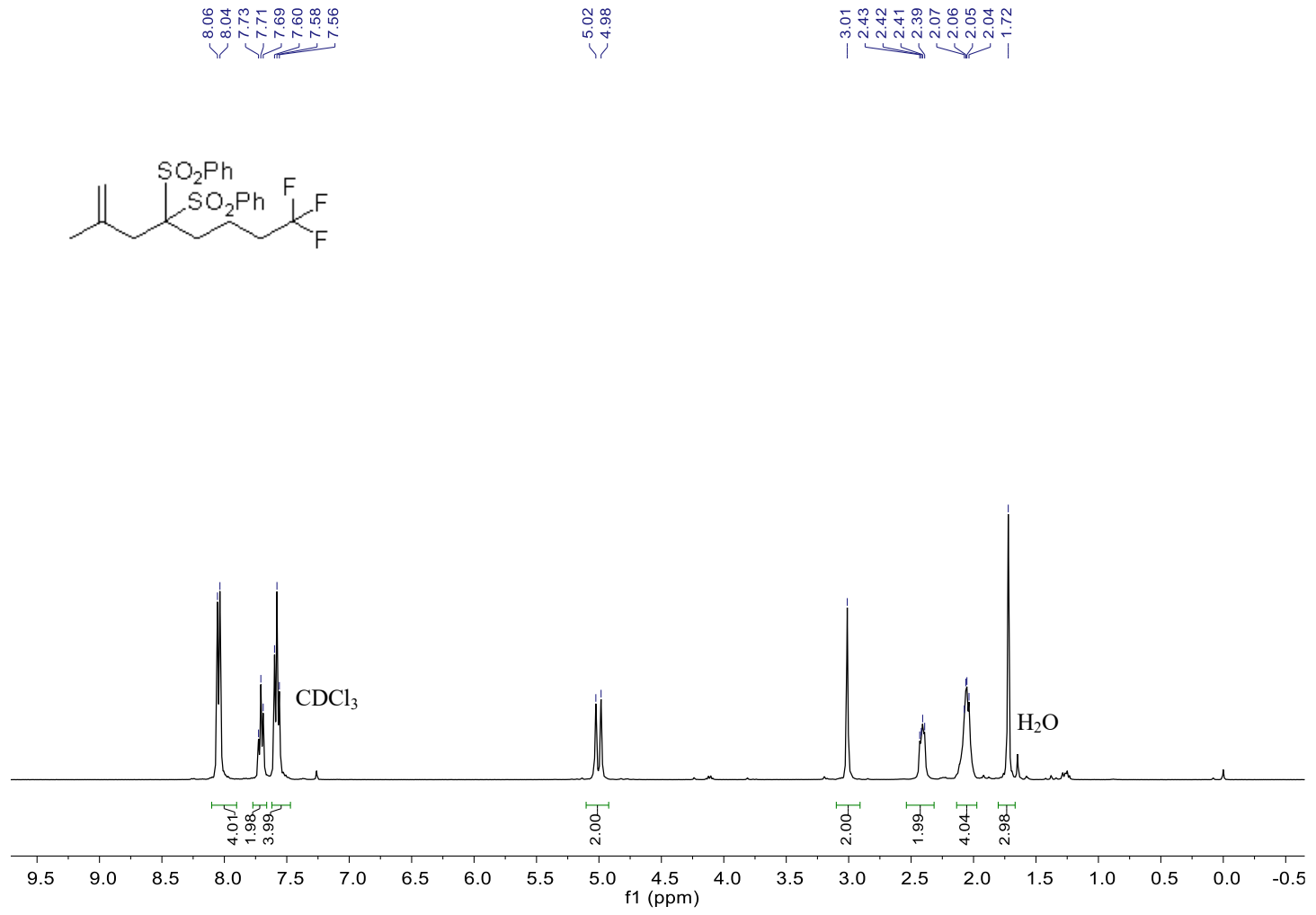

${ }^{13} \mathrm{C}$ NMR (151 MHz, $\left.\mathrm{CDCl}_{3}\right)$<smiles>C=C(C)CC(CC(CC(F)(F)F)(SO)c1ccccc1)(SO)S(=O)(=O)c1ccccc1</smiles>

$\mathrm{CDCl}_{3}$

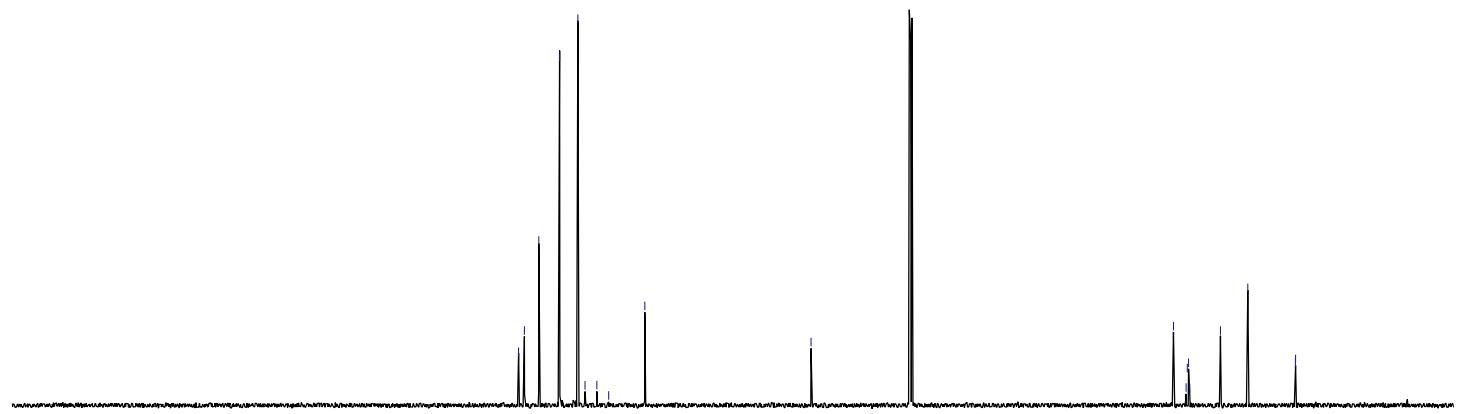

$\begin{array}{lllllllllllllllllllll}210 & 200 & 190 & 180 & 170 & 160 & 150 & 140 & 130 & 120 & \begin{array}{c}110 \\ \mathrm{f} 1(\mathrm{ppm})\end{array} & \begin{array}{l}100 \\ 90\end{array} & 80 & 70 & 60 & 50 & 40 & 30 & 20 & 10 & 0\end{array}$ 


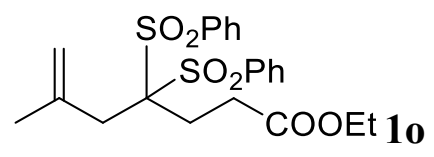

${ }^{1} \mathrm{H}$ NMR (400 MHz, $\left.\mathrm{CDCl}_{3}\right)$

$\underbrace{-1}$

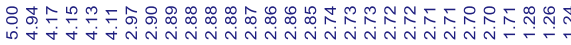
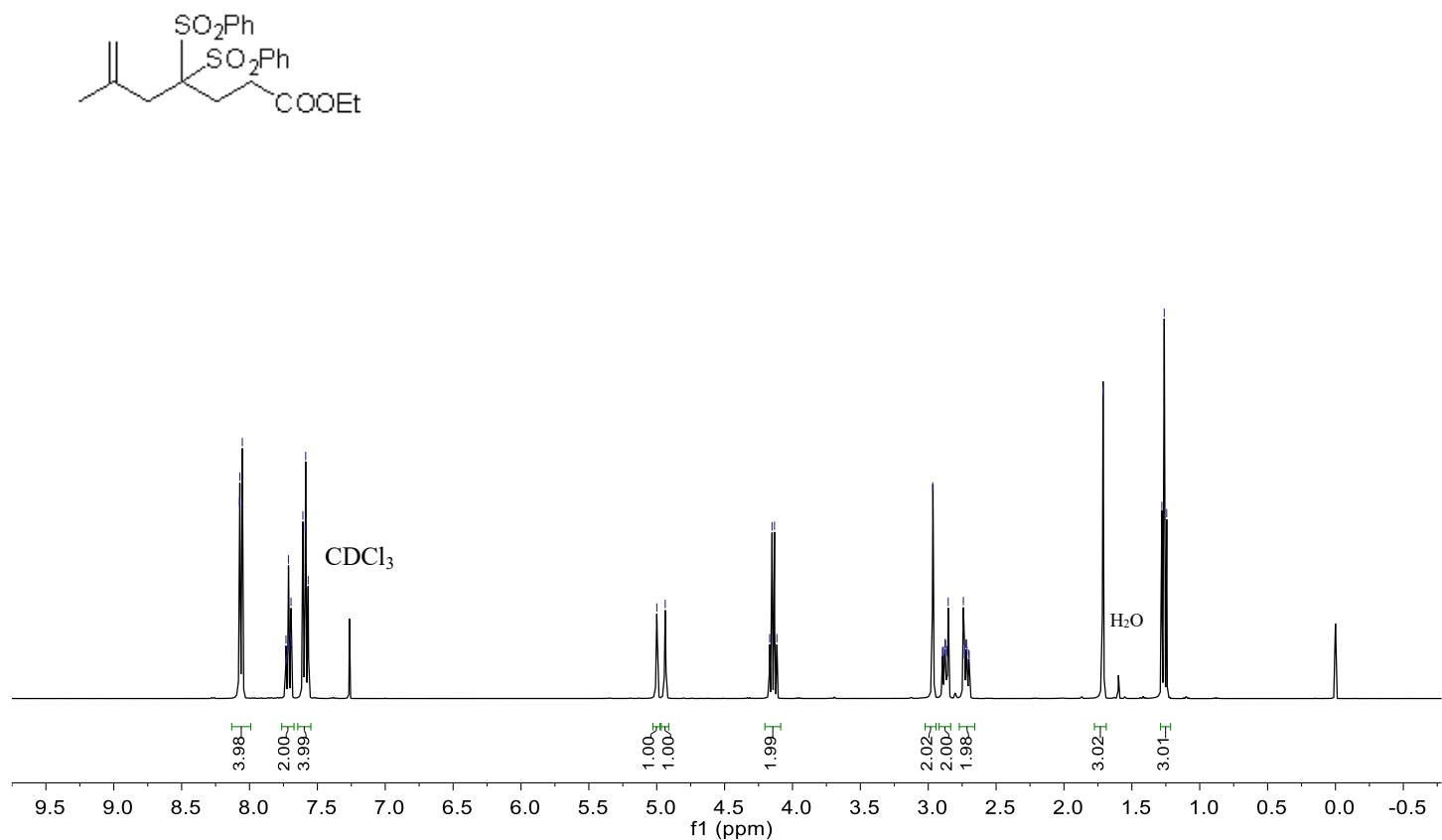

${ }^{13} \mathrm{C}$ NMR (101 MHz, $\left.\mathrm{CDCl}_{3}\right)$

垔

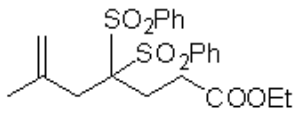

$\mathrm{CDCl}_{3}$

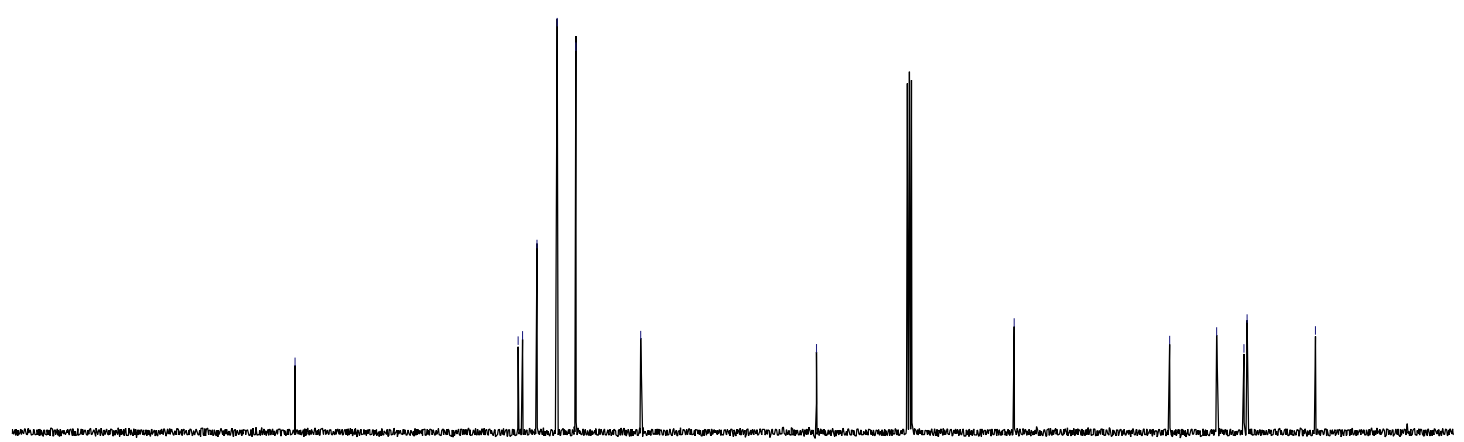

$\begin{array}{lllllllllllllllllllll}210 & 200 & 190 & 180 & 170 & 160 & 150 & 140 & 130 & 120 & \begin{array}{c}110 \\ \mathrm{f} 1(\mathrm{ppm})\end{array} & 90 & 80 & 70 & 60 & 50 & 40 & 30 & 20 & 10 & 0\end{array}$ 


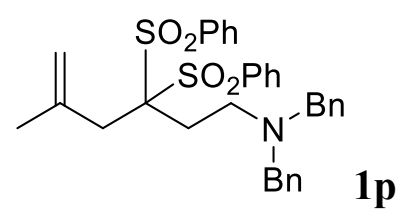

${ }^{1}$ H NMR (400 MHz, CDCl 3$)$

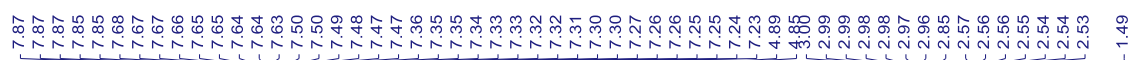<smiles>C=C(C)CC([Se])([Se])CC(O)(c1ccccc1)N(Cc1ccccc1)c1ccccc1</smiles>

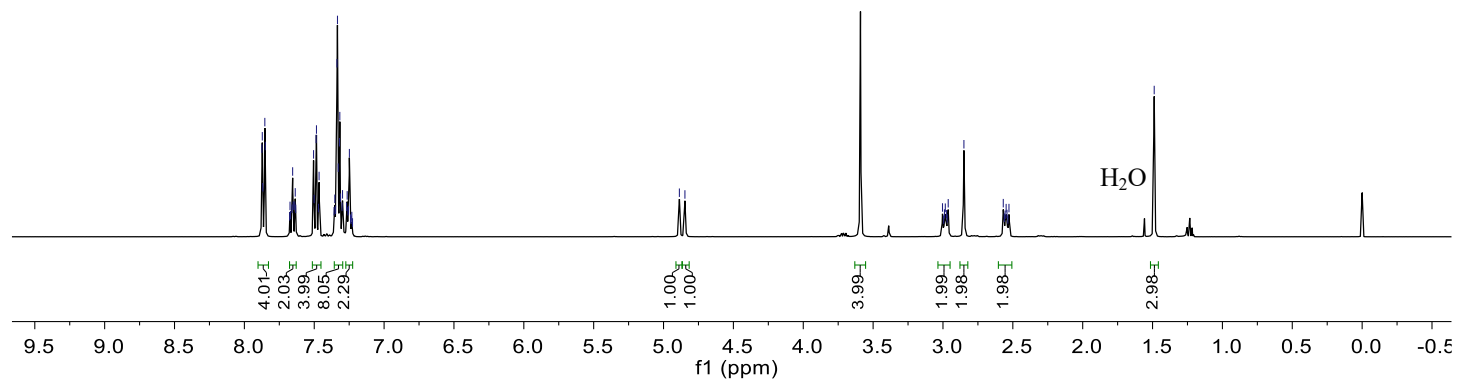

\section{${ }^{13} \mathrm{C}$ NMR (101 MHz, $\left.\mathrm{CDCl}_{3}\right)$}

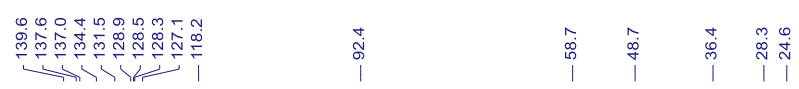<smiles>C=C(C)CC1(S)CC(c2ccccc2)(N(Cc2ccccc2)c2ccccc2)S(=O)(=O)O1</smiles>

$\mathrm{CDCl}_{3}$

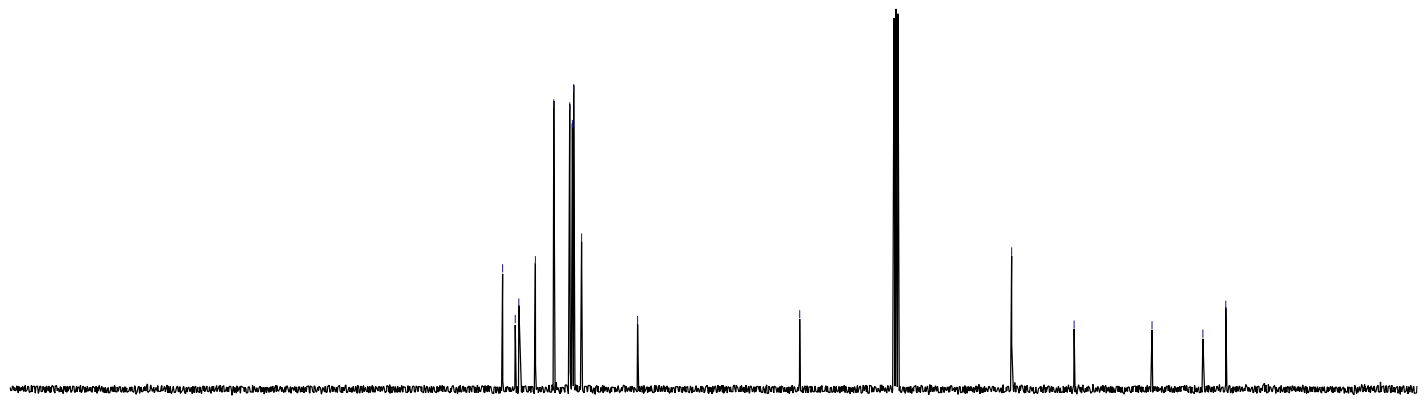

$\begin{array}{llllllllllllllllllllll}210 & 200 & 190 & 180 & 170 & 160 & 150 & 140 & 130 & 120 & 110 & 100 & 90 & 80 & 70 & 60 & 50 & 40 & 30 & 20 & 10 & 0\end{array}$ 


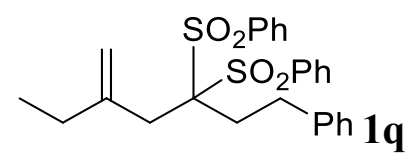

${ }^{1}$ H NMR (400 MHz, $\left.\mathrm{CDCl}_{3}\right)$

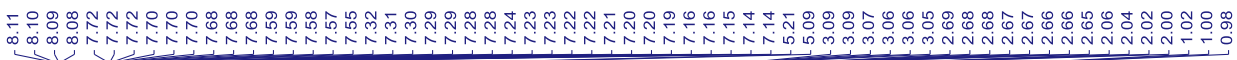
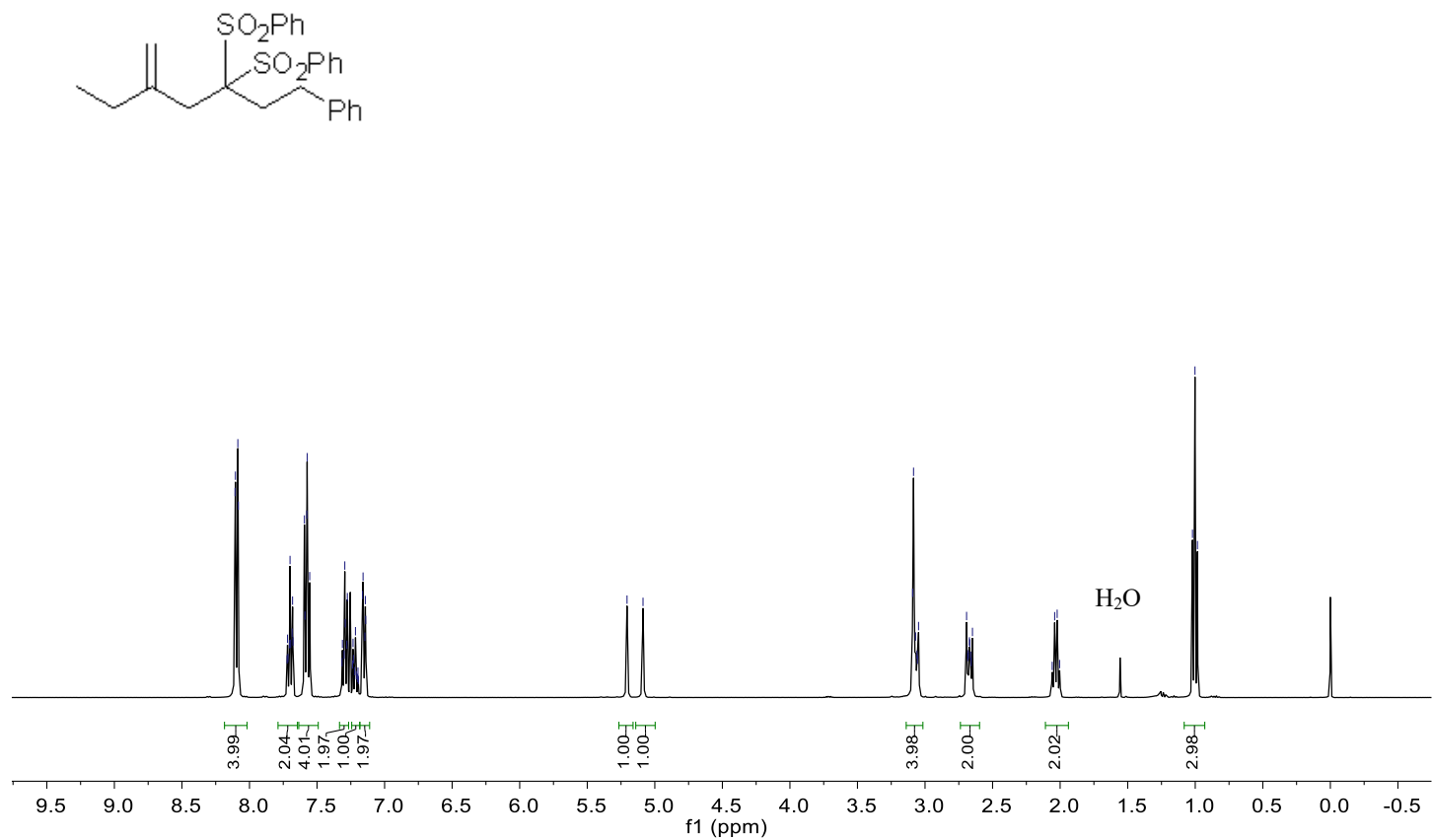

${ }^{13} \mathrm{C}$ NMR (101 MHz, CDCl 3$)$

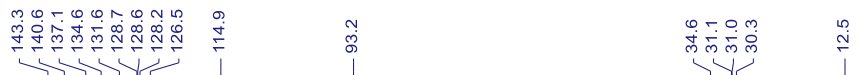<smiles>C=C(CC)CC(CC(C)([Se])c1ccccc1)(c1ccccc1)c1ccccc1</smiles>

$\mathrm{CDCl}_{3}$

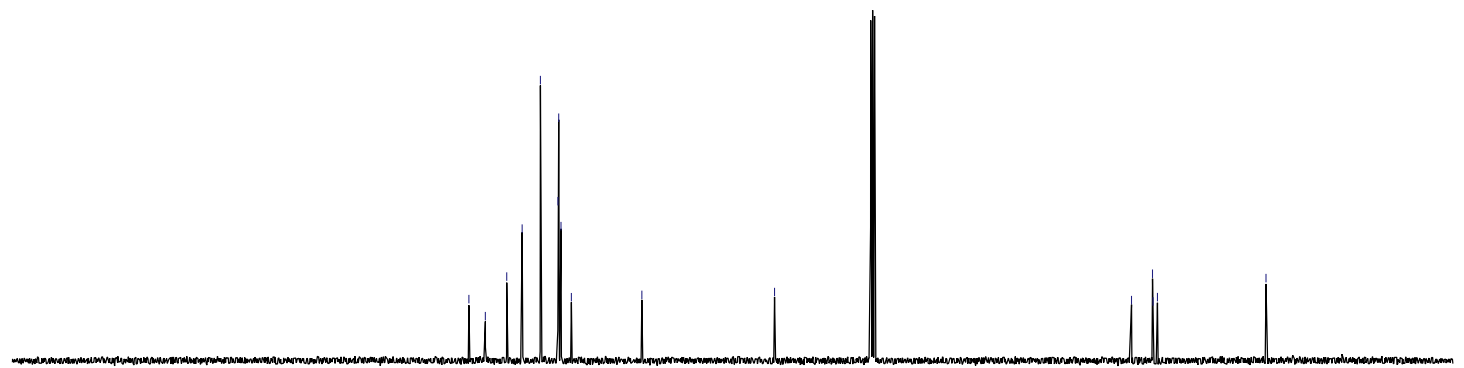

$\begin{array}{lllllllllllllllllllllll}210 & 200 & 190 & 180 & 170 & 160 & 150 & 140 & 130 & 120 & 110 & 100 & 90 & 80 & 70 & 60 & 50 & 40 & 30 & 20 & 10 & 0 & -10\end{array}$ 


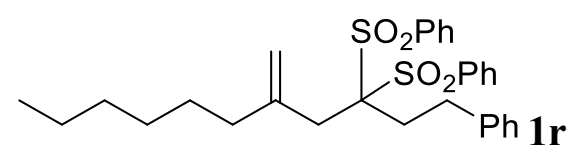

\section{${ }^{1}$ H NMR (400 MHz, CDCl $\left.\mathbf{C l}_{3}\right)$}

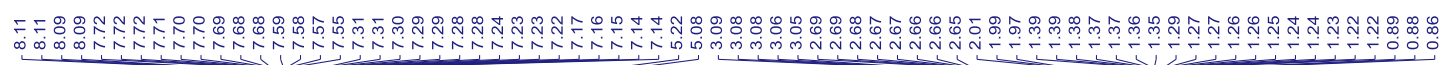
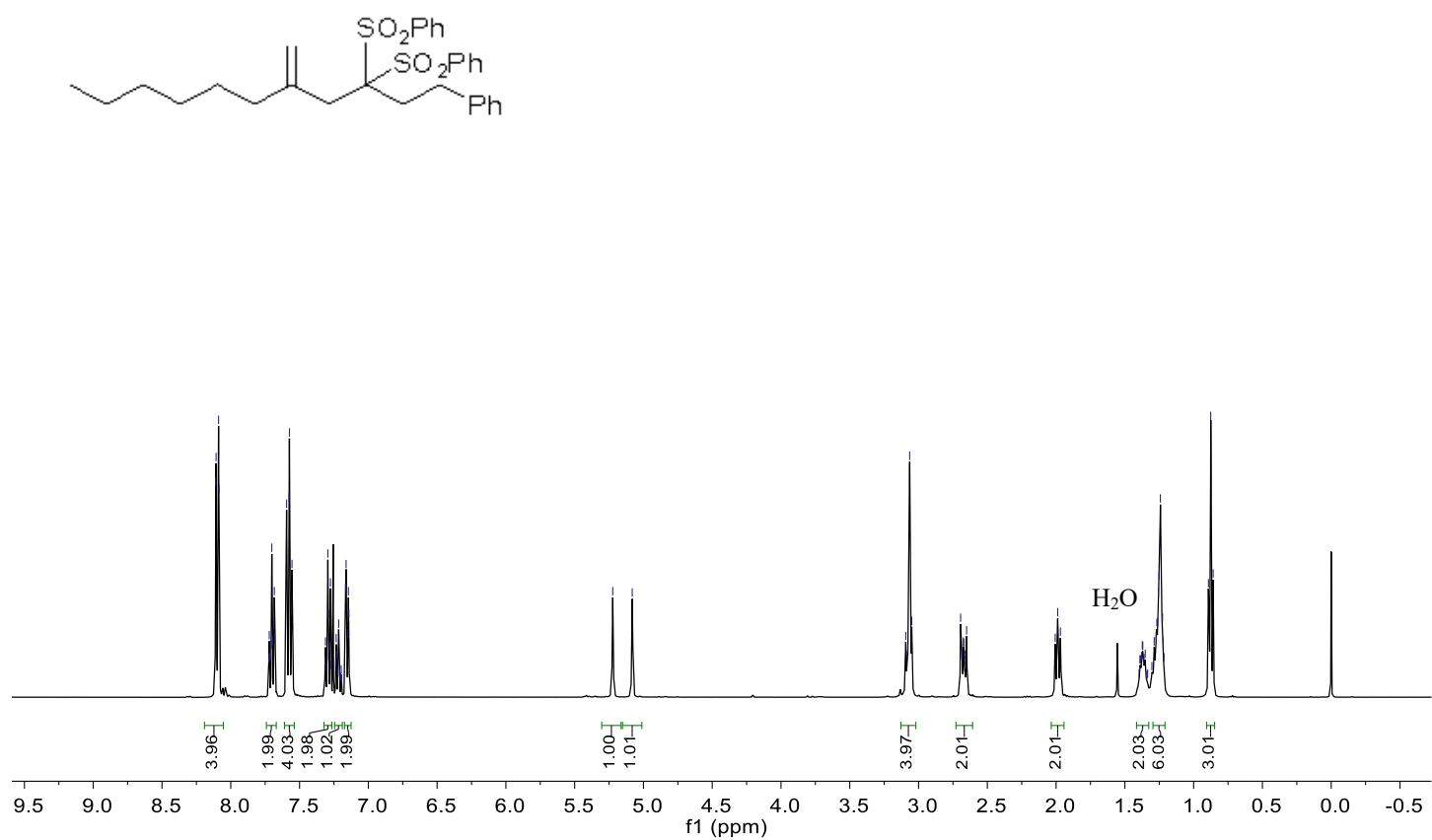

${ }^{13} \mathrm{C}$ NMR (101 MHz, $\left.\mathrm{CDCl}_{3}\right)$

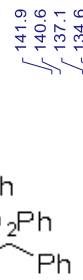

$\mathrm{CDCl}_{3}$

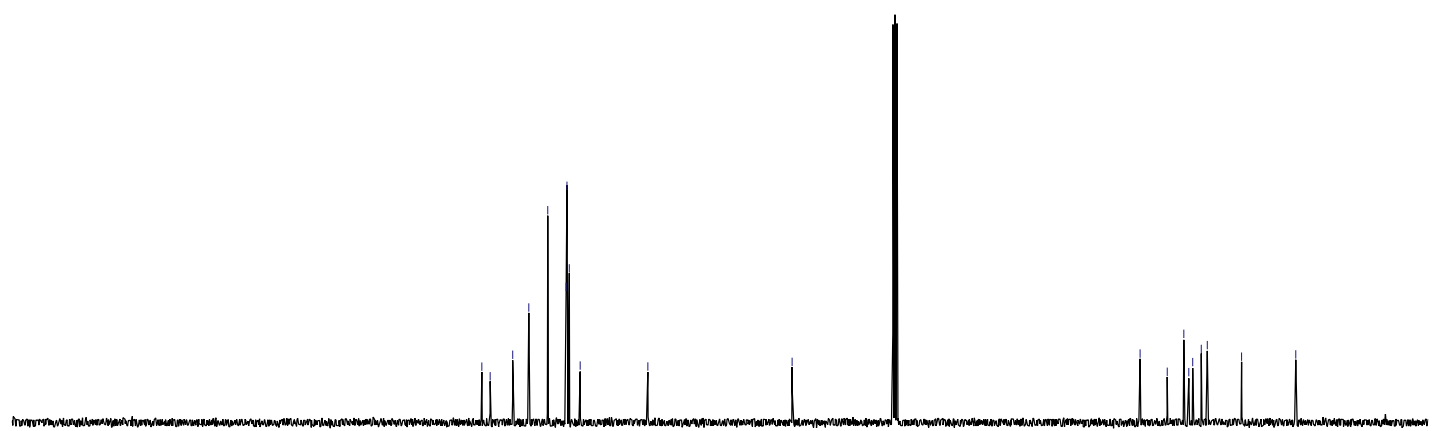

$\begin{array}{lllllllllllllllllllll}10 & 200 & 190 & 180 & 170 & 160 & 150 & 140 & 130 & 120 & \begin{array}{c}110 \\ \mathrm{f} 1(\mathrm{ppm})\end{array} & 90 & 80 & 70 & 60 & 50 & 40 & 30 & 20 & 10 & 0\end{array}$ 


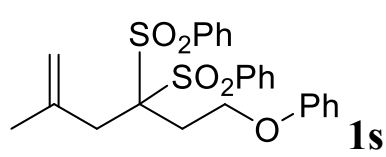

${ }^{1} \mathrm{H}$ NMR (400 MHz, $\left.\mathrm{CDCl}_{3}\right)$

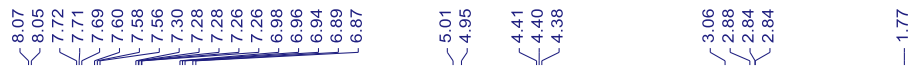
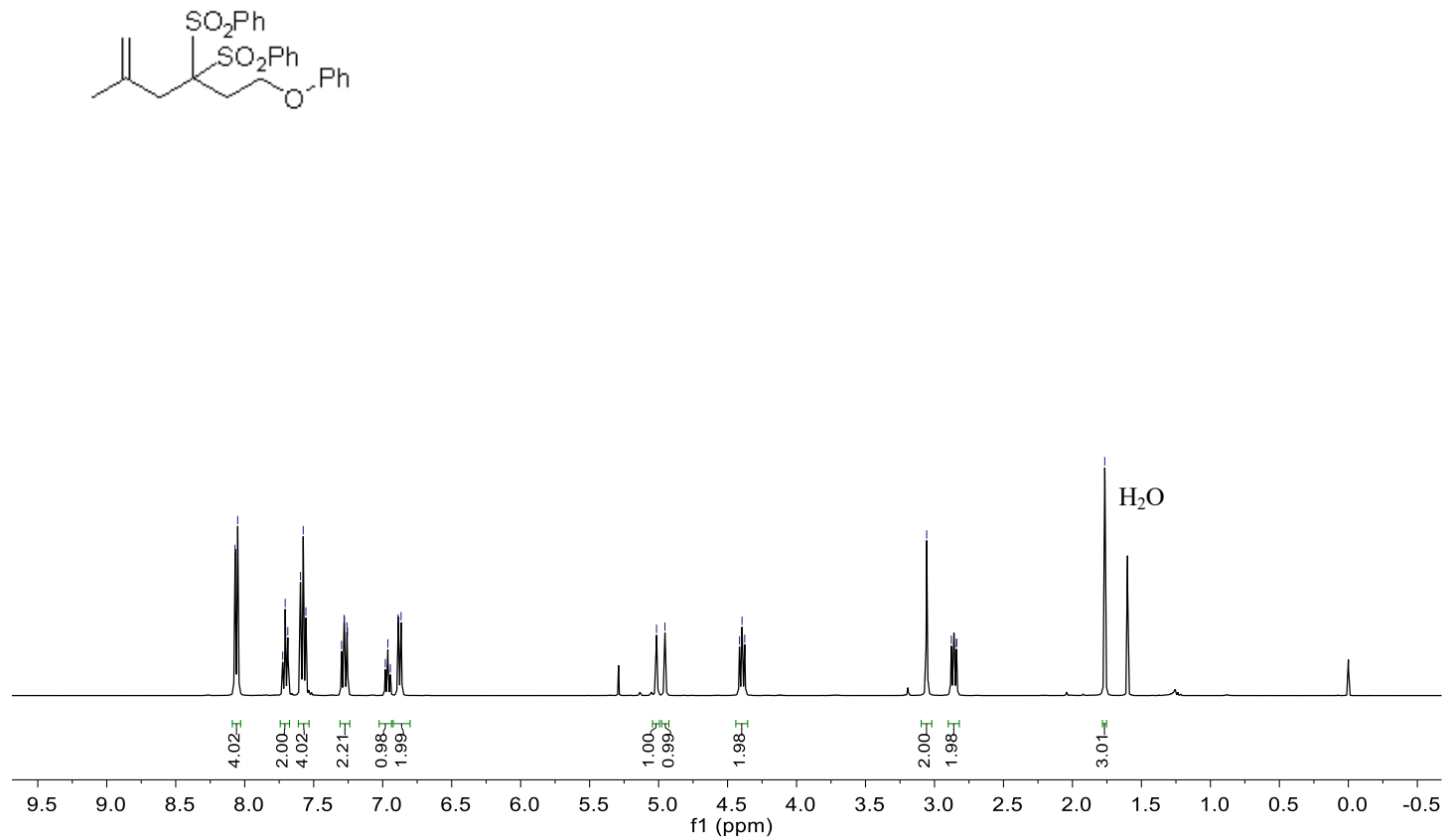

${ }^{13} \mathrm{C}$ NMR (101 MHz, $\left.\mathrm{CDCl}_{3}\right)$

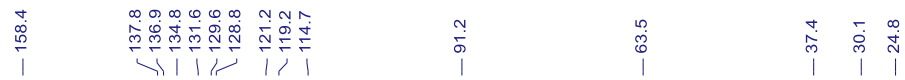

$\| \overbrace{\mathrm{O}^{-\mathrm{Ph}}}^{\mathrm{SO}_{2} \mathrm{Ph}}$

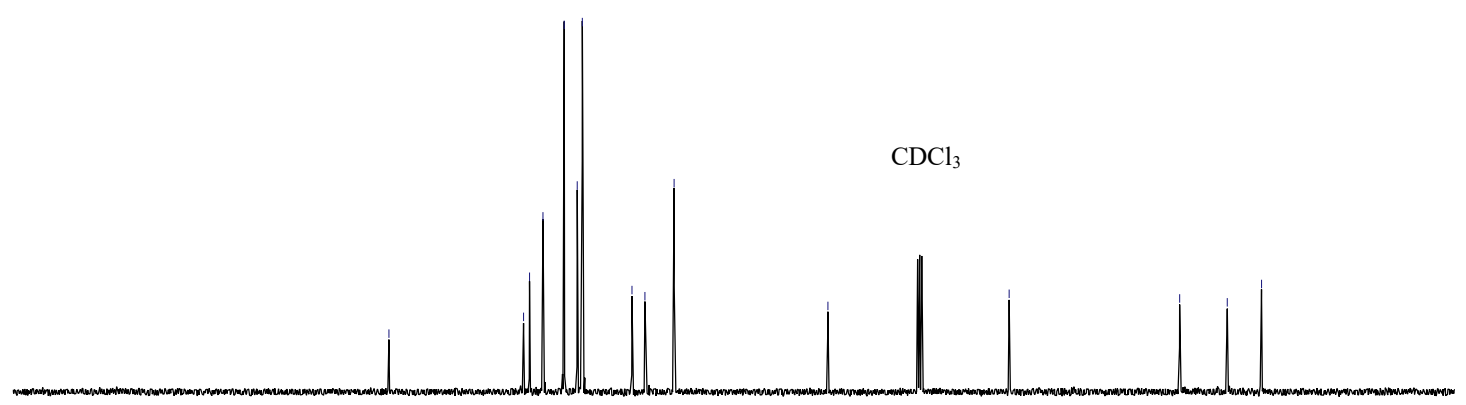

$\begin{array}{lllllllllllllllllllll}210 & 200 & 190 & 180 & 170 & 160 & 150 & 140 & 130 & 120 & \begin{array}{l}110 \\ \mathrm{f} 1(\mathrm{ppm})\end{array} & 90 & 80 & 70 & 60 & 50 & 40 & 30 & 20 & 10 & 0\end{array}$ 


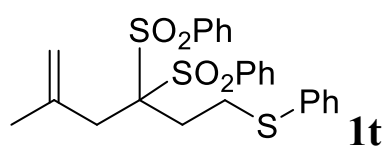

${ }^{1} \mathrm{H}$ NMR (400 MHz, $\left.\mathrm{CDCl}_{3}\right)$

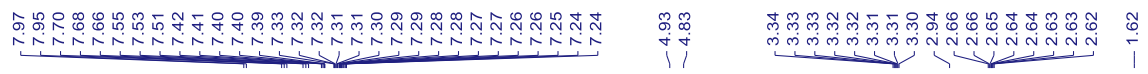<smiles>C=C(C)CC(C)(CC(=S)Sc1ccccc1)S(=O)(=O)c1ccccc1</smiles>

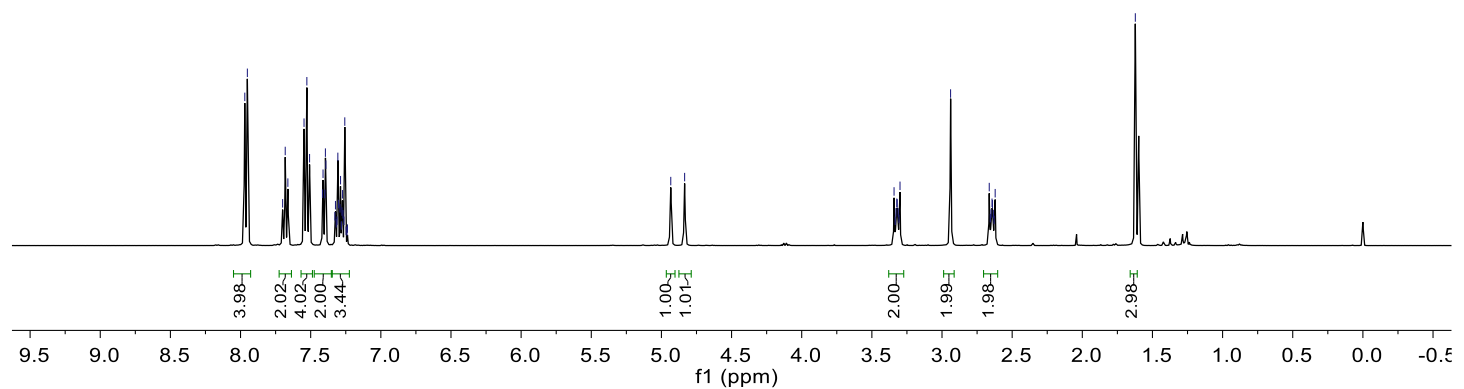

${ }^{13} \mathrm{C}$ NMR (101 MHz, $\left.\mathrm{CDCl}_{3}\right)$

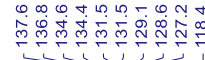

$\mathrm{CDCl}_{3}$

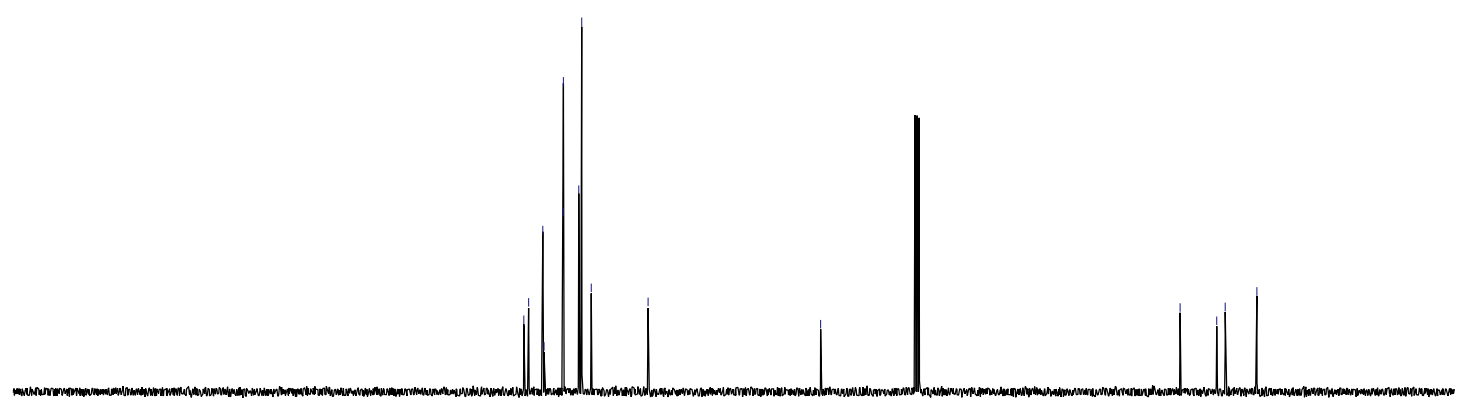

$\begin{array}{lllllllllllllllllllll}210 & 200 & 190 & 180 & 170 & 160 & 150 & 140 & 130 & 120 & \begin{array}{c}110 \\ \mathrm{f} 1(\mathrm{ppm})\end{array} & 100 & 80 & 70 & 60 & 50 & 40 & 30 & 20 & 10 & 0\end{array}$ 
$\overbrace{\mathrm{Ph} 1 \mathrm{u}}$

${ }^{1} \mathrm{H}$ NMR (400 MHz, $\left.\mathrm{CDCl}_{3}\right)$

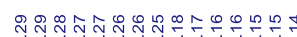

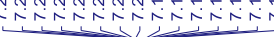<smiles>C=C(C)CCCCc1ccccc1</smiles>

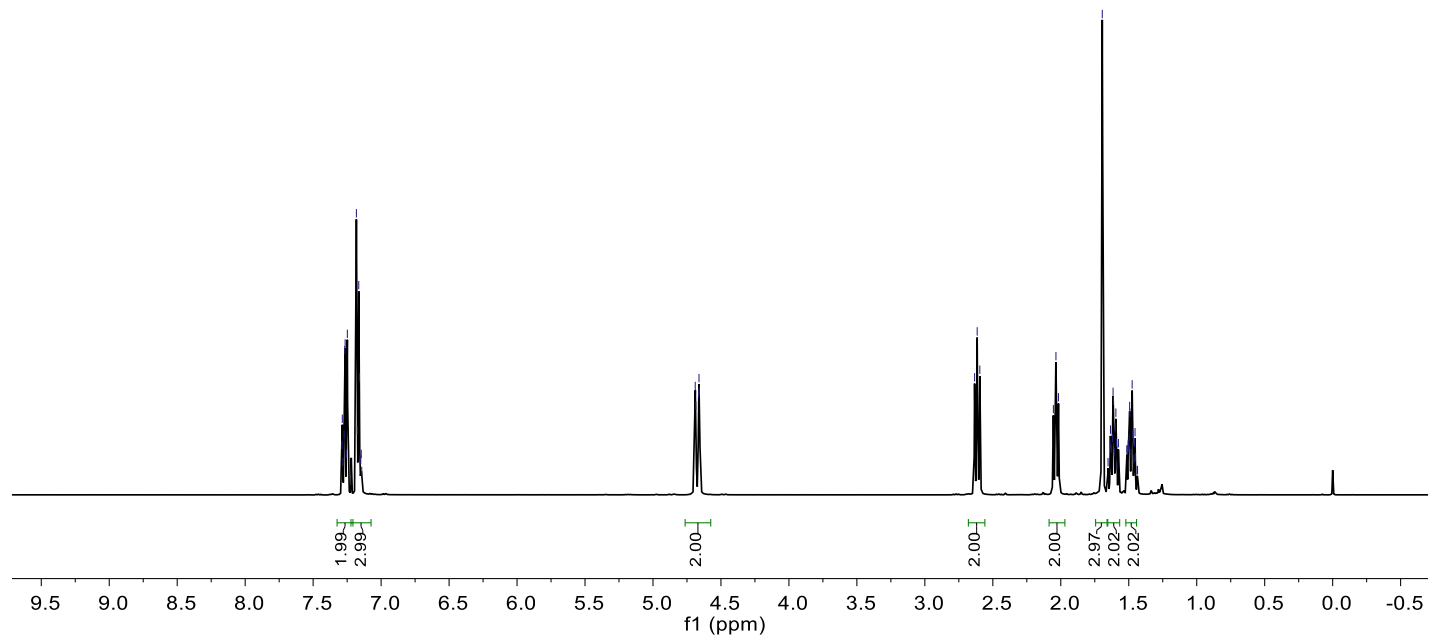

${ }^{13} \mathrm{C}$ NMR (101 MHz, $\left.\mathrm{CDCl}_{3}\right)$<smiles>C=C(C)CCCCc1ccccc1</smiles>

$\mathrm{CDCl}_{3}$

$\begin{array}{lllllllllllllllllllll}210 & 200 & 190 & 180 & 170 & 160 & 150 & 140 & 130 & 120 & \begin{array}{r}110 \\ \mathrm{f} 1(\mathrm{ppm})\end{array} & \begin{array}{l}100 \\ 80\end{array} & 80 & 70 & 60 & 50 & 40 & 30 & 20 & 10 & 0\end{array}$ 
$\underbrace{\mathrm{OHC}}_{\mathrm{SO}_{2} \mathrm{Ph}} \stackrel{\mathrm{Me}}{\mathrm{SO}_{2} \mathrm{Ph}}$

${ }^{1} \mathrm{H}$ NMR (400 MHz, $\left.\mathrm{CDCl}_{3}\right)$

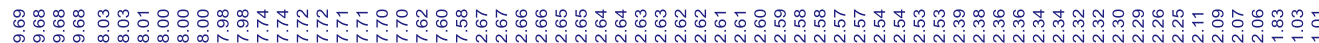

$\mathrm{N}_{\mathrm{SO}_{2} \mathrm{Ph}}^{\mathrm{OHC}}$

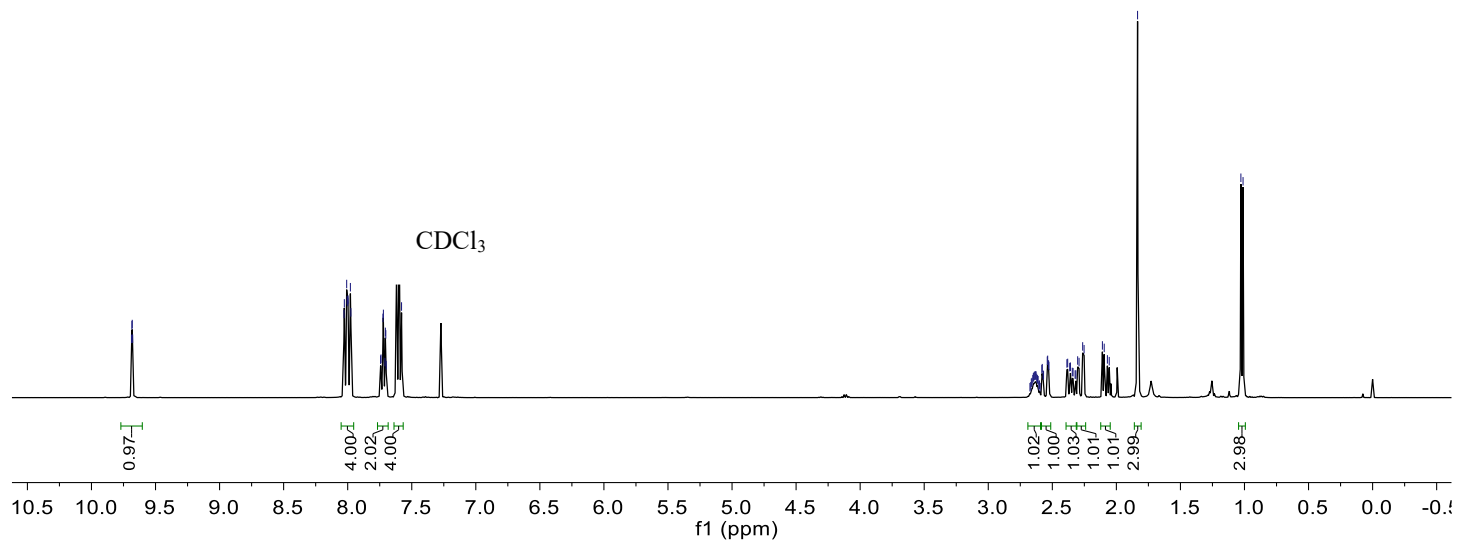

${ }^{13} \mathrm{C}$ NMR (101 MHz, CDCl 3$)$

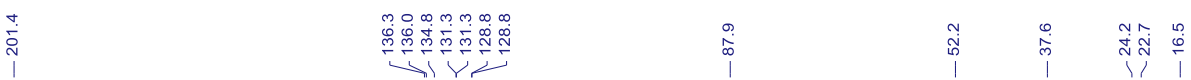

- $\mathrm{Me}_{\mathrm{SO}_{2} \mathrm{Ph}}^{\mathrm{OHC}}$

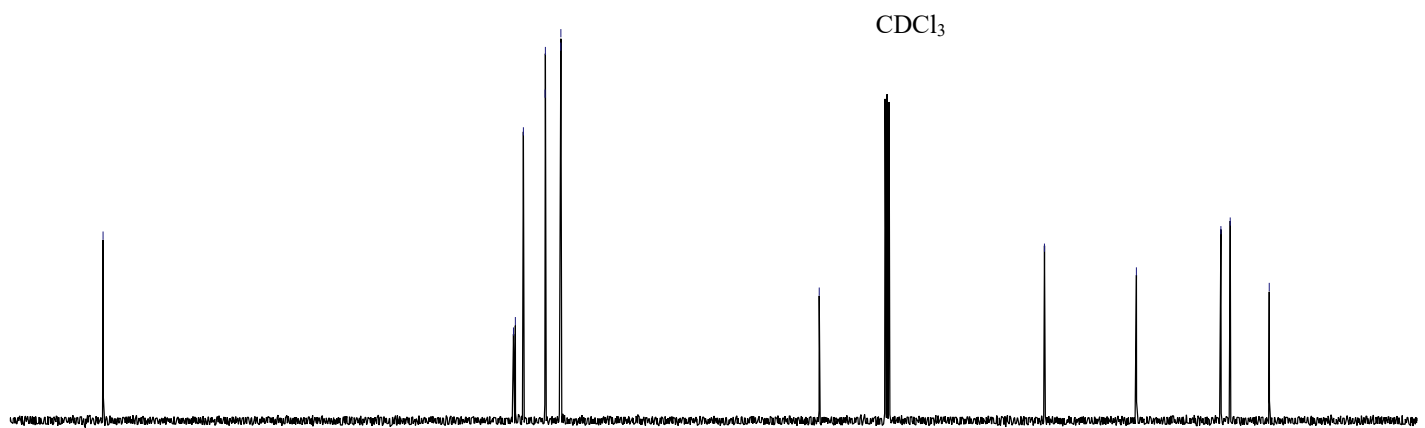

$\begin{array}{llllllllllllllllllllll}210 & 200 & 190 & 180 & 170 & 160 & 150 & 140 & 130 & 120 & 110 & 100 & 90 & 80 & 70 & 60 & 50 & 40 & 30 & 20 & 10 & 0\end{array}$ 
$\underbrace{\mathrm{OHC}}_{\mathrm{SO}_{2} \mathrm{Ph}_{2} \mathbf{b}}$

${ }^{1} \mathrm{H}$ NMR (400 MHz, $\left.\mathrm{CDCl}_{3}\right)$

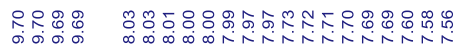

$\sim_{\mathrm{SO}_{2} \mathrm{Ph}}^{\mathrm{EHC}}$

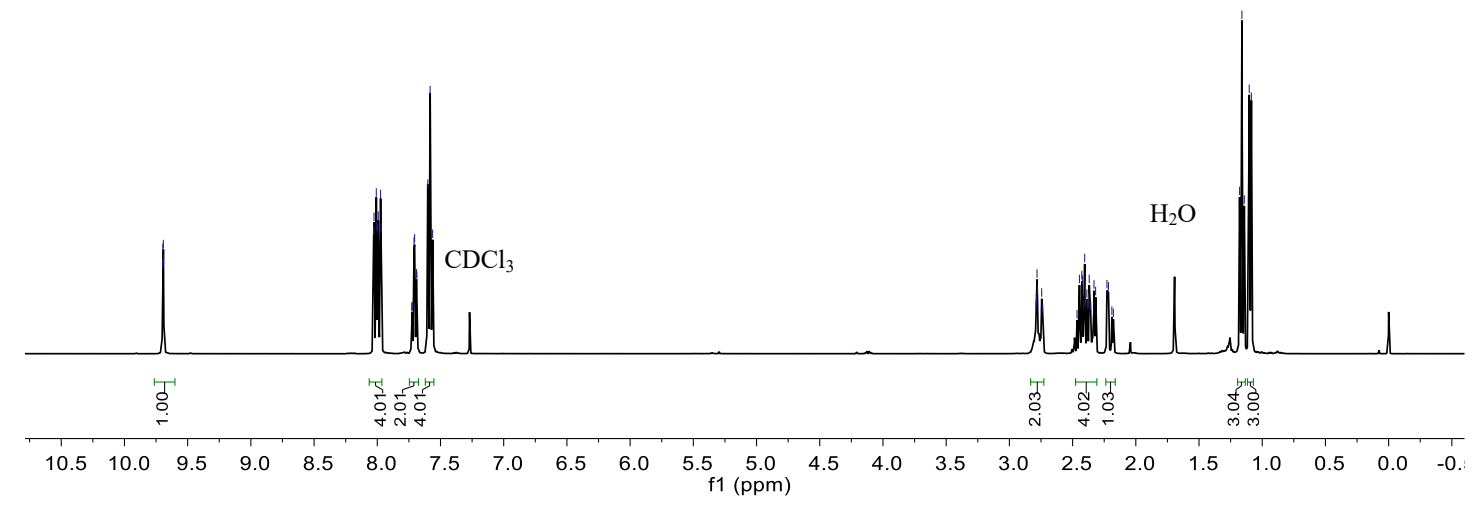

${ }^{13} \mathrm{C}$ NMR (101 MHz, $\left.\mathrm{CDCl}_{3}\right)$

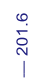

$2 \mathrm{~L}_{\mathrm{SO}_{2} \mathrm{Ph}}^{\mathrm{OHC}}$

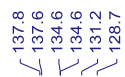

$\mathrm{CDCl}_{3}$

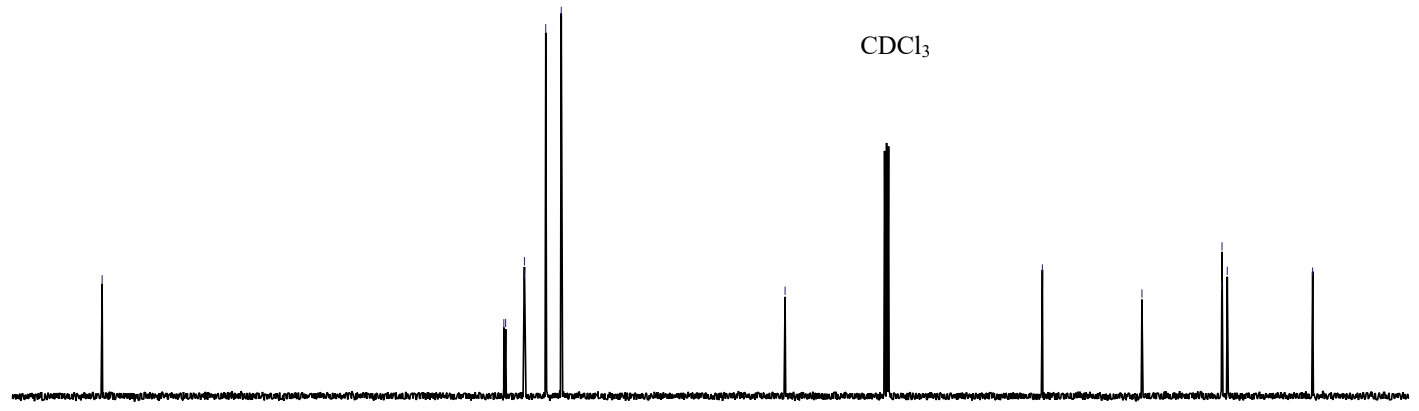

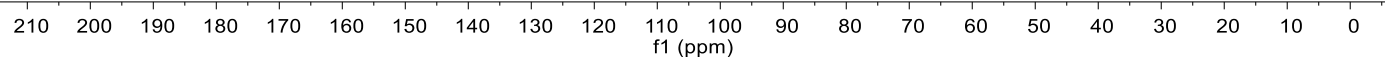




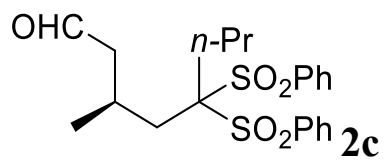

\section{${ }^{1} \mathrm{H}$ NMR (600 MHz, $\left.\mathrm{CDCl}_{3}\right)$}

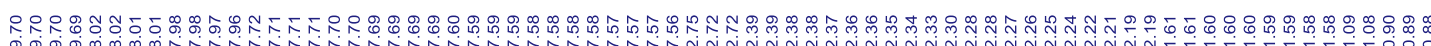<smiles></smiles>

$\mathrm{CDCl}_{3}$

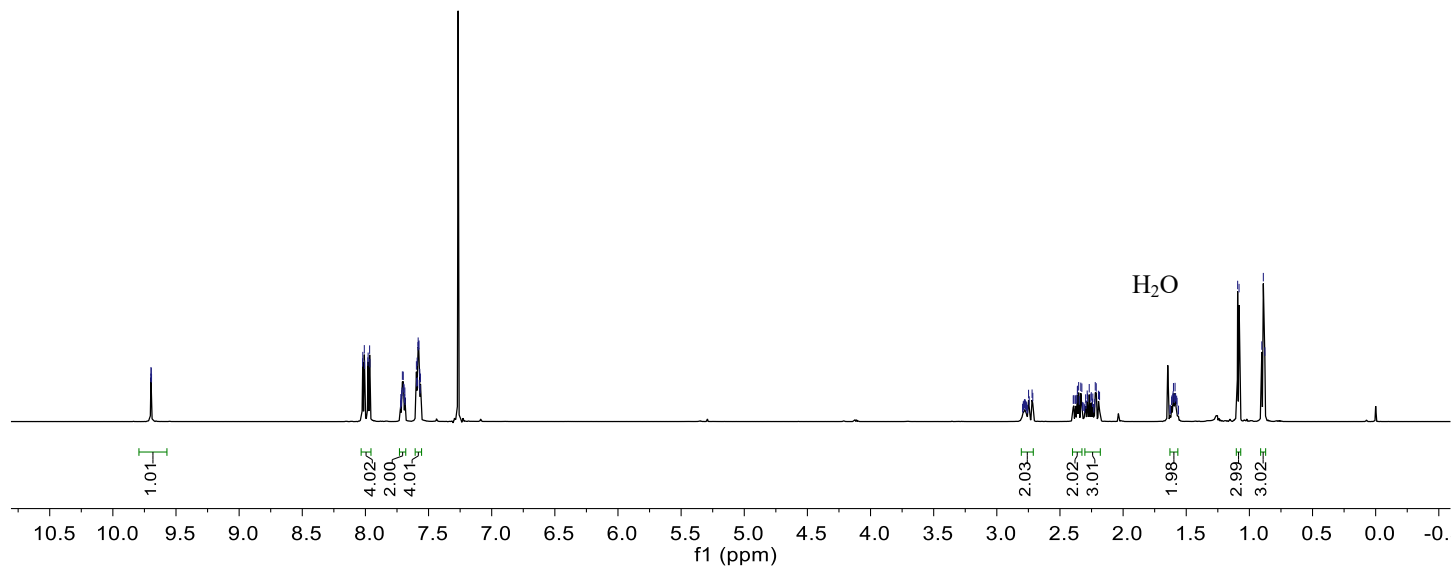

\section{${ }^{13} \mathrm{C}$ NMR (151 MHz, CDCl 3$)$}<smiles>[CH-]C=[AsH2]</smiles>

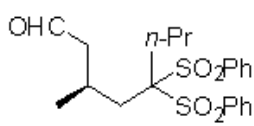

$\mathrm{CDCl}_{3}$

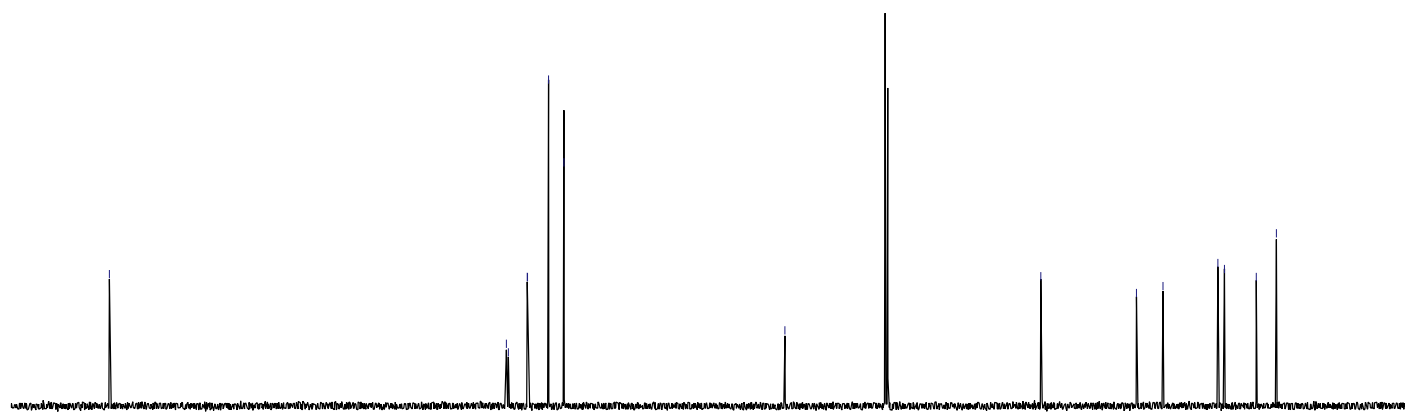

$\begin{array}{lllllllllll}210 & 200 & 190 & 180 & 170 & 160 & 150 & 140 & 130 & 120 & \begin{array}{l}110 \\ \mathrm{f} 1(\mathrm{ppm})\end{array}\end{array}$ 


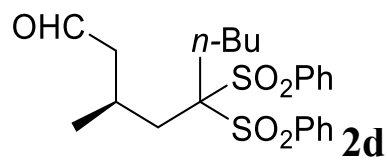

\section{${ }^{1} \mathrm{H}$ NMR (400 MHz, $\left.\mathrm{CDCl}_{3}\right)$}

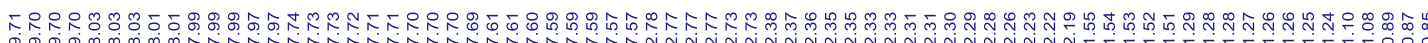

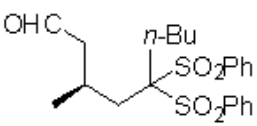

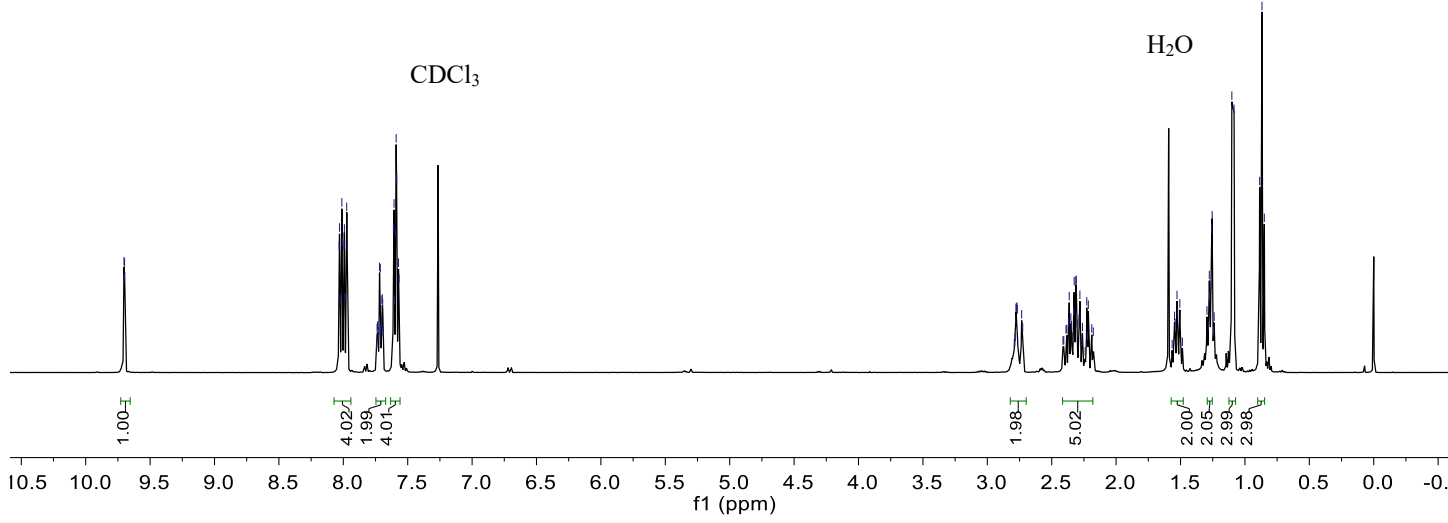

${ }^{13} \mathrm{C}$ NMR (101 MHz, $\left.\mathrm{CDCl}_{3}\right)$<smiles>C=[Sn]</smiles><smiles>CCOC(CC(C)CC=O)(Oc1ccccc1)Oc1ccccc1</smiles>

$\mathrm{CDCl}_{3}$

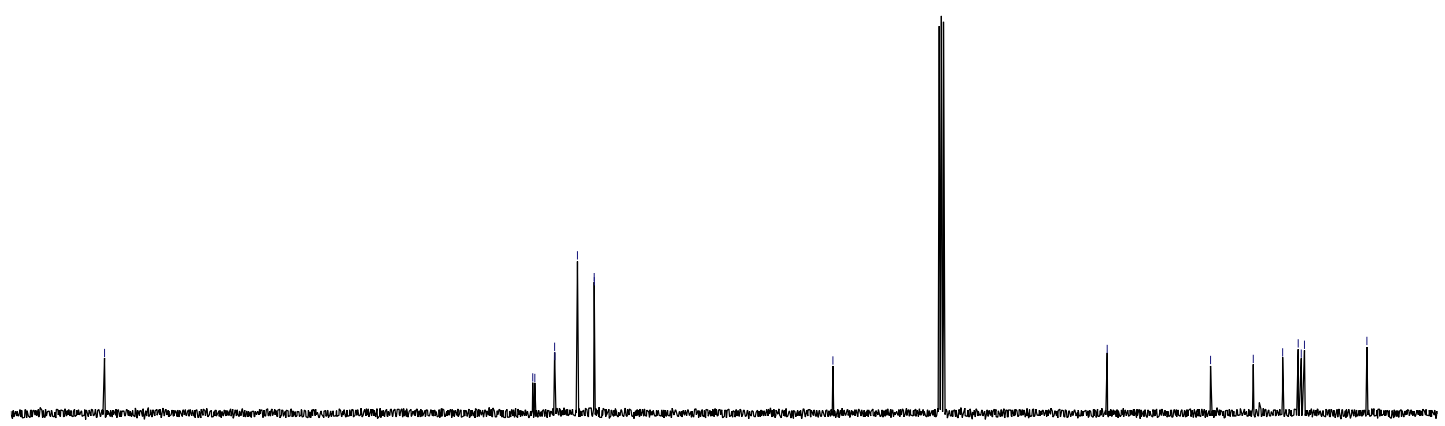

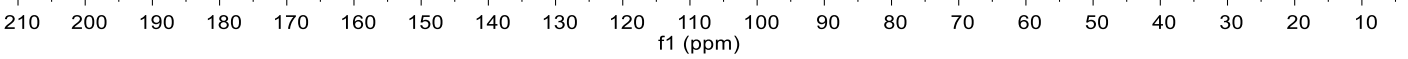


<smiles></smiles>

${ }^{1} \mathrm{H}$ NMR (400 MHz, $\left.\mathrm{CDCl}_{3}\right)$

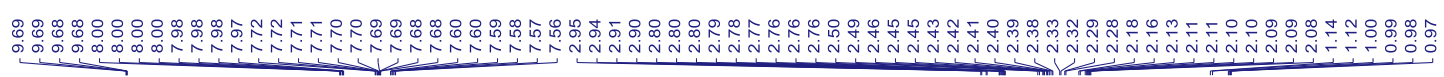
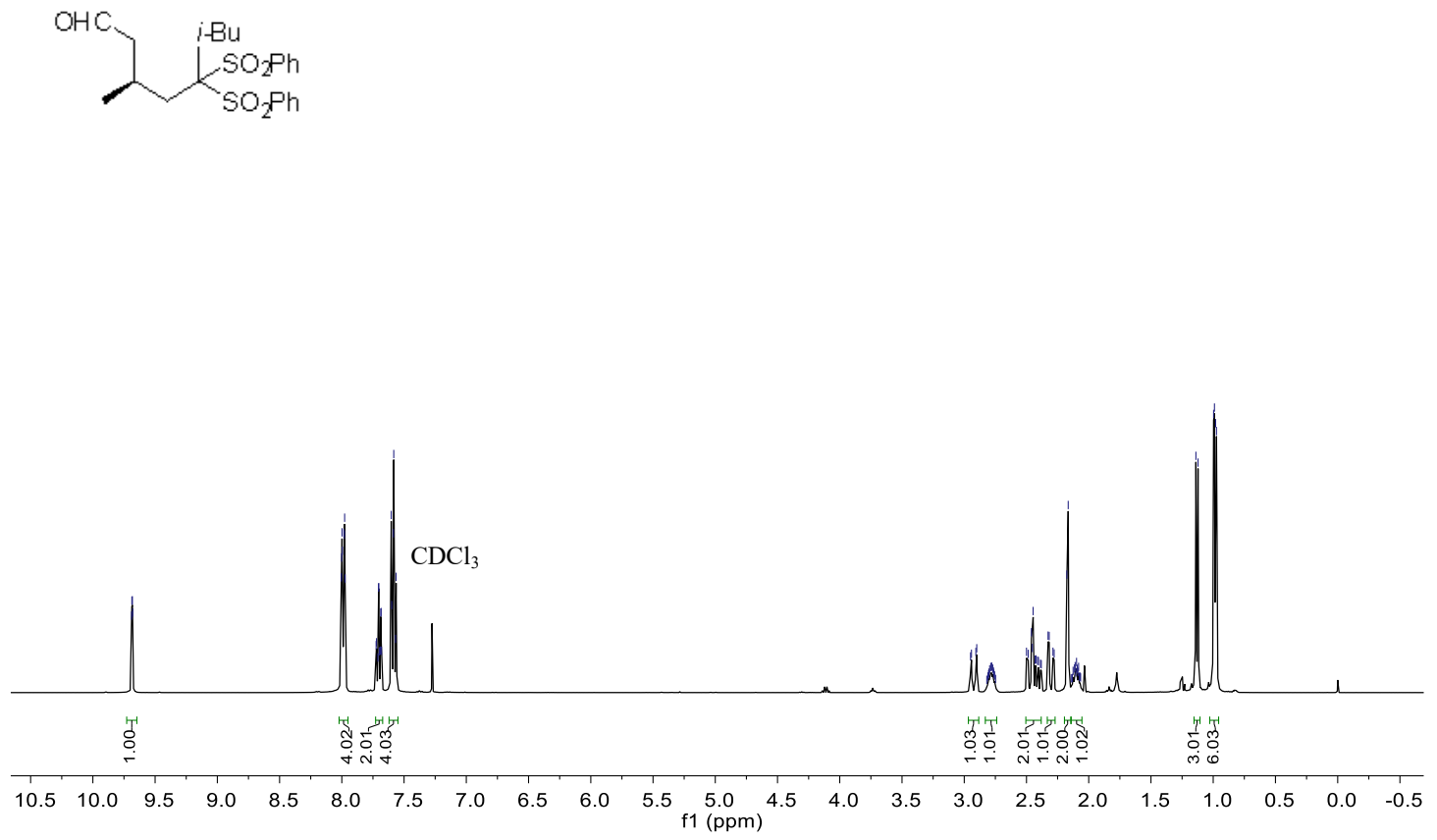

${ }^{13} \mathrm{C}$ NMR (101 MHz, $\left.\mathrm{CDCl}_{3}\right)$

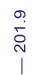

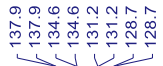

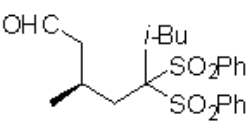

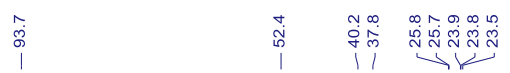

$\mathrm{CDCl}_{3}$
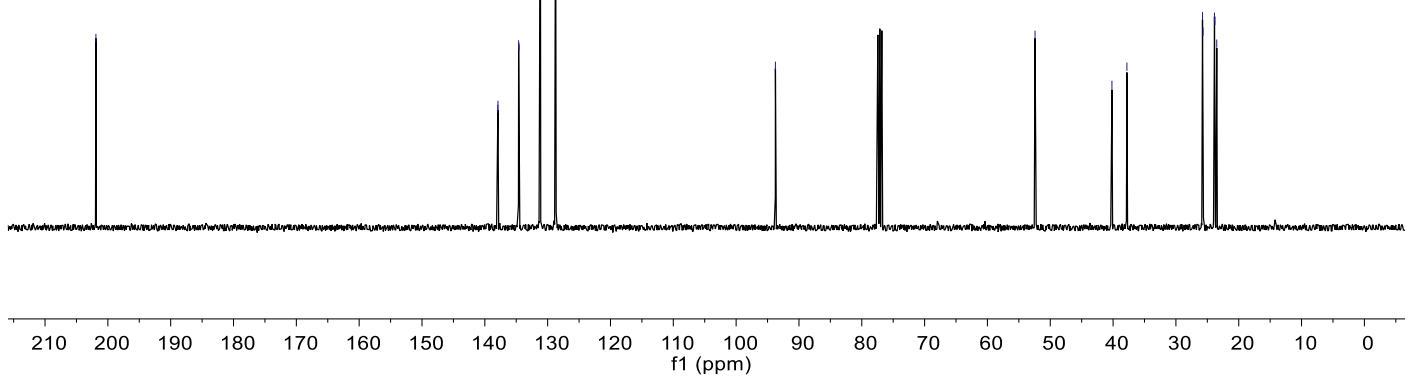

$\mathrm{S} 53$ 


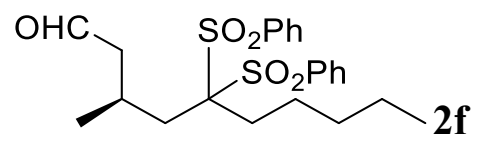

\section{${ }^{1} \mathrm{H}$ NMR (400 MHz, $\left.\mathrm{CDCl}_{3}\right)$}

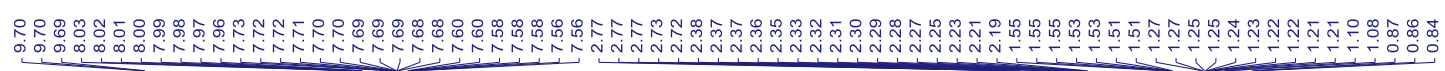

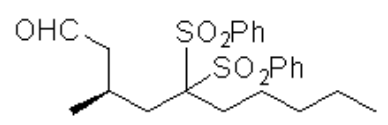

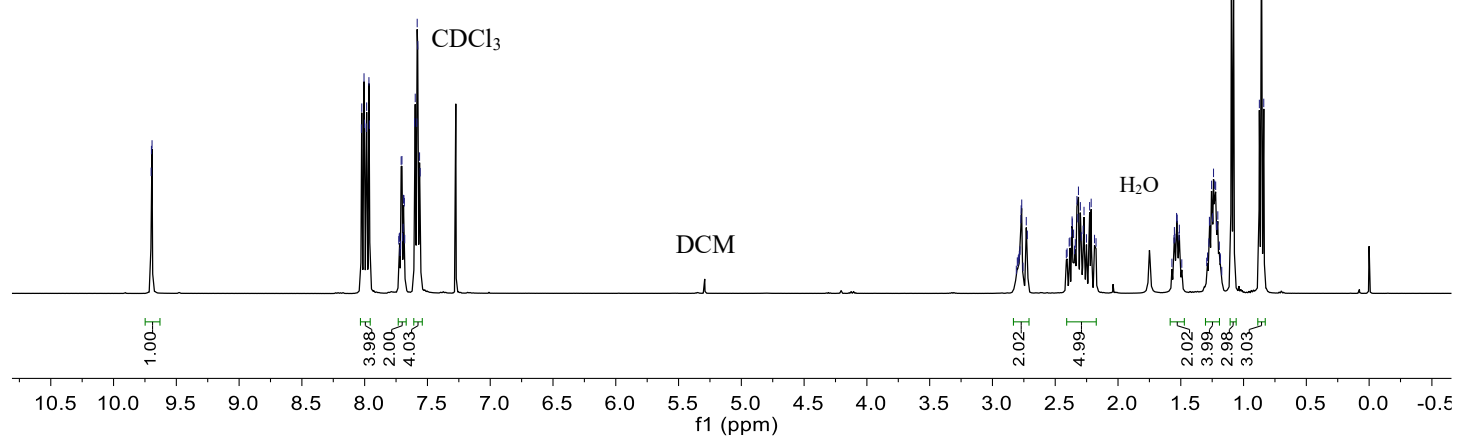

\section{${ }^{13} \mathrm{C}$ NMR (101 MHz, $\left.\mathrm{CDCl}_{3}\right)$}
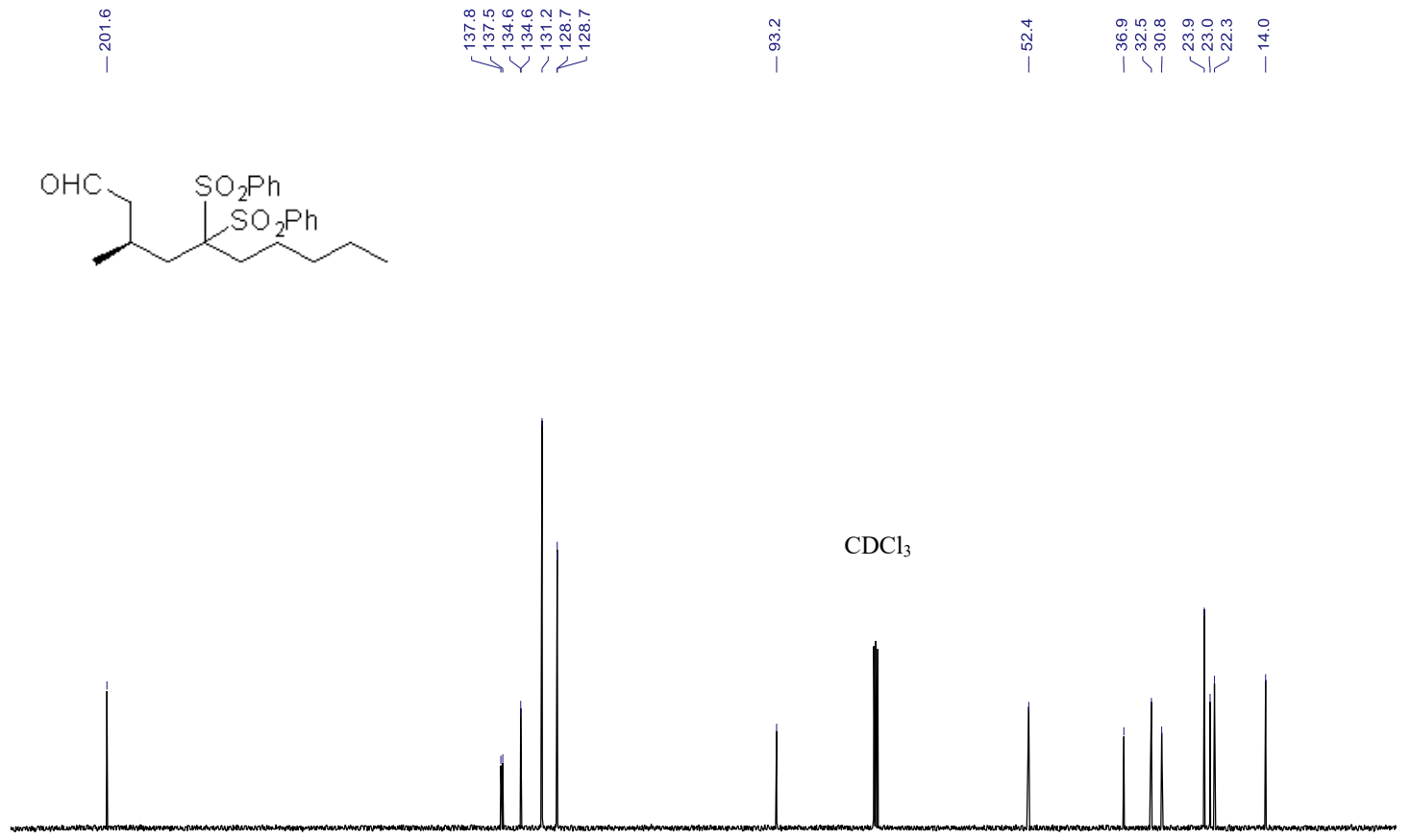

$\begin{array}{lllllllllllllllllllll}210 & 200 & 190 & 180 & 170 & 160 & 150 & 140 & 130 & 120 & \begin{array}{c}110 \\ \mathrm{f} 1(\mathrm{ppm})\end{array} & 100 & 80 & 70 & 60 & 50 & 40 & 30 & 20 & 10 & 0\end{array}$ 


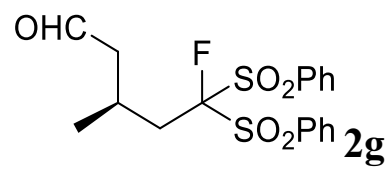

${ }^{1} \mathrm{H}$ NMR (600 MHz, $\left.\mathrm{CDCl}_{3}\right)$

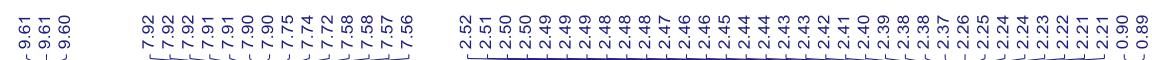

$\overbrace{\mathrm{SO}_{2} \mathrm{Ph}}^{\mathrm{SO}_{2} \mathrm{Ph}}$

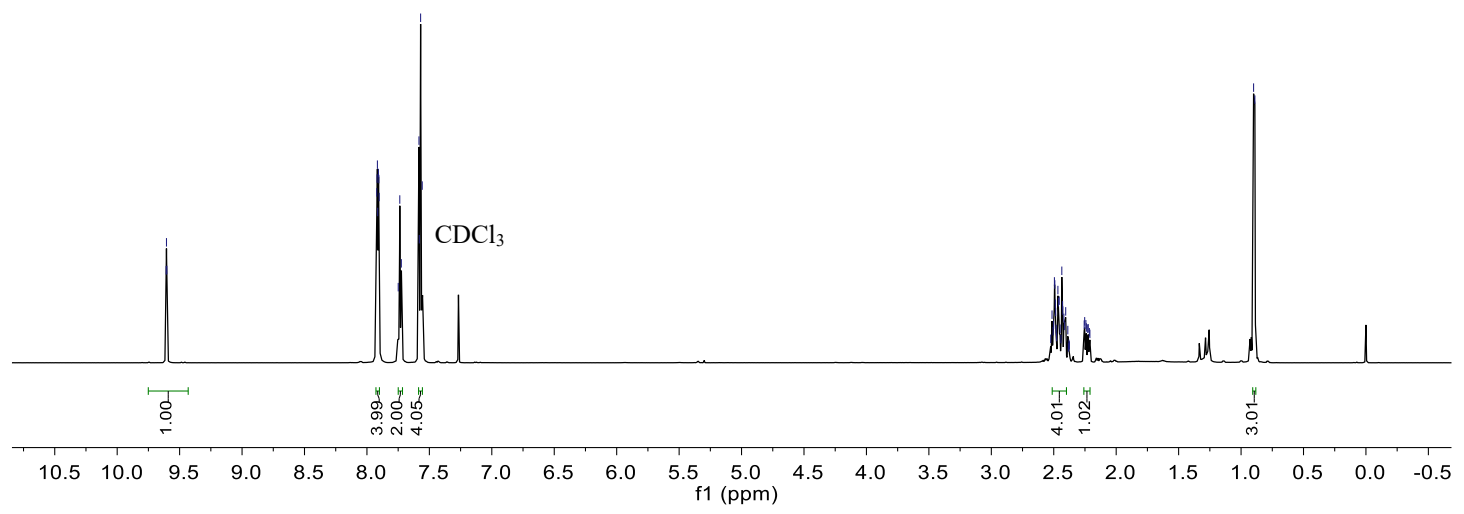

${ }^{13} \mathrm{C}$ NMR (151 MHz, CDCl 3$)$

$\stackrel{\substack{\dot{0} \\ \text { i }}}{i}$

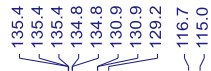

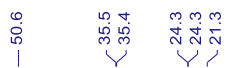

$\underbrace{\mathrm{OHC}}_{\mathrm{SO}_{2} \mathrm{Ph}}$

$\mathrm{CDCl}_{3}$

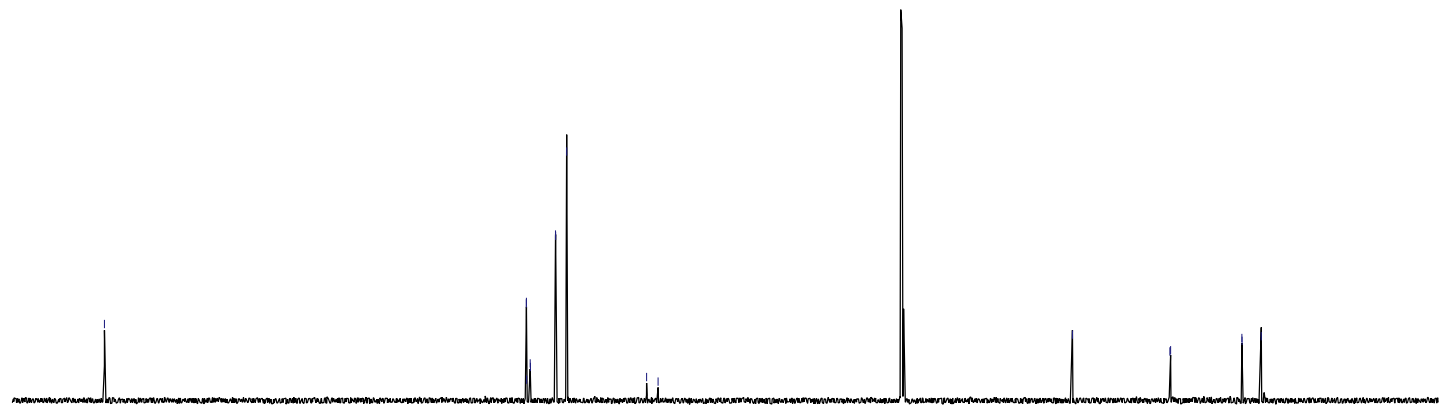

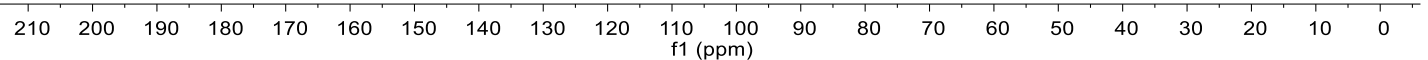


${ }^{19}$ F NMR (565 MHz, $\left.\mathrm{CDCl}_{3}\right)$<smiles>CC(CC=O)CC(F)([Se]Oc1ccccc1)[Se]Oc1ccccc1</smiles>

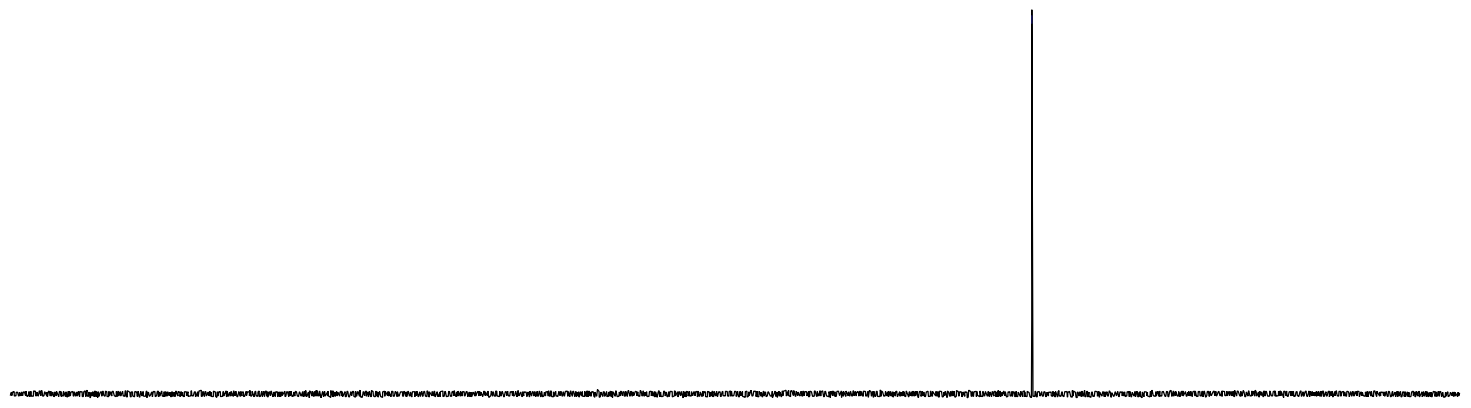

$\begin{array}{lllllllllllllllllllllllllll}10 & 0 & -10 & -20 & -30 & -40 & -50 & -60 & -70 & -80 & -90 & -100 & -110 & -120 & -130 & -140 & -150 & -160 & -170 & -180 & -190 & -200 & -210\end{array}$ 
<smiles>CC(C)=C(CC(CC(C)CC=O)(S(=O)(=O)c1ccccc1)S(=O)(=O)c1ccccc1)C(C)C</smiles>

${ }^{1} \mathrm{H}$ NMR (400 MHz, $\mathrm{CDCl}_{3}$ )

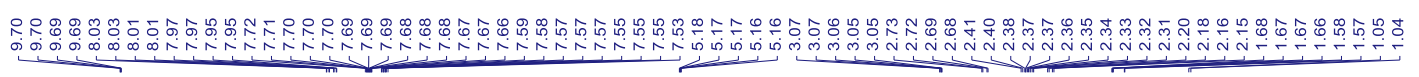
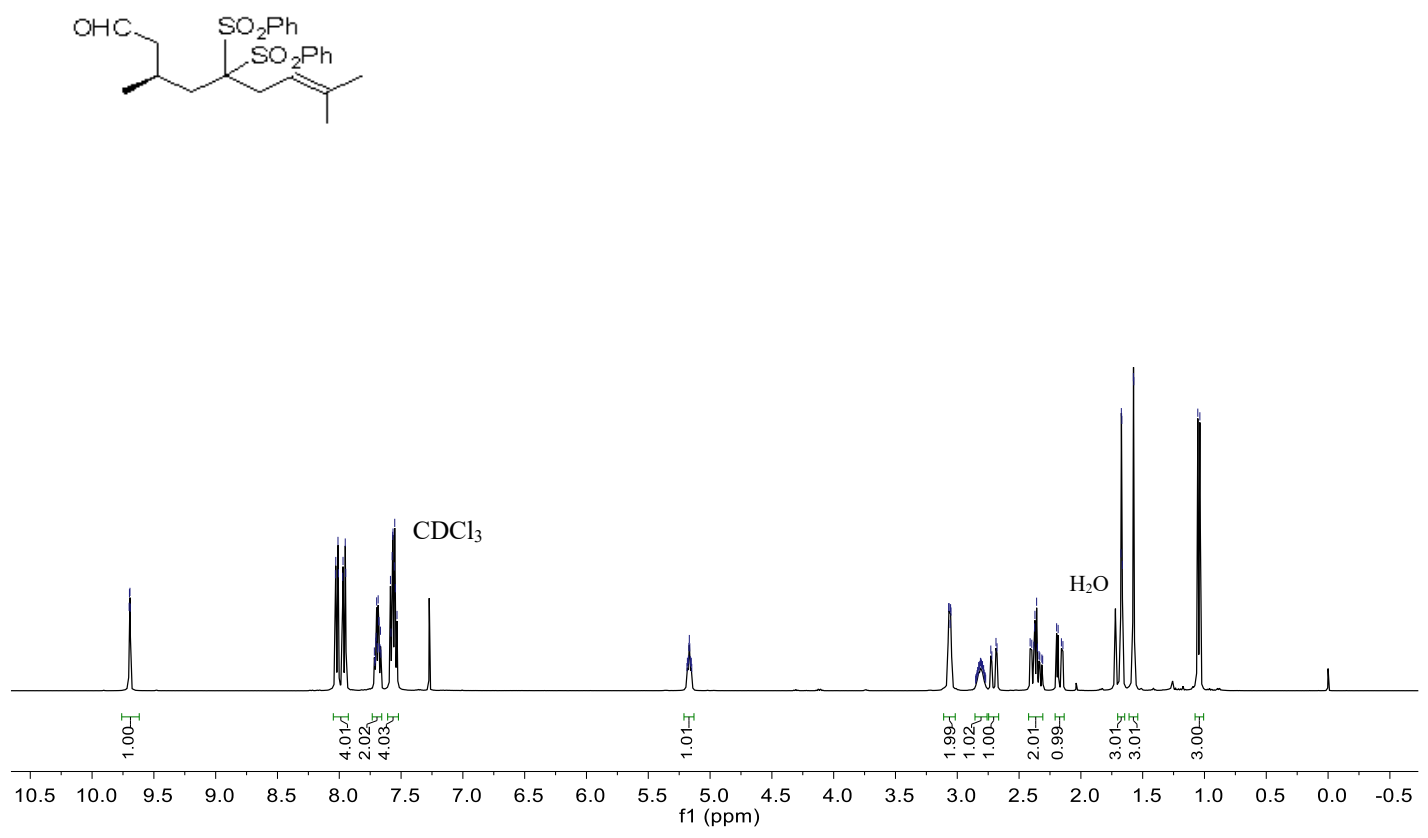

${ }^{13} \mathrm{C}$ NMR (101 MHz, $\left.\mathrm{CDCl}_{3}\right)$

io

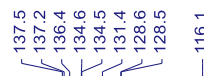

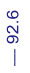

i
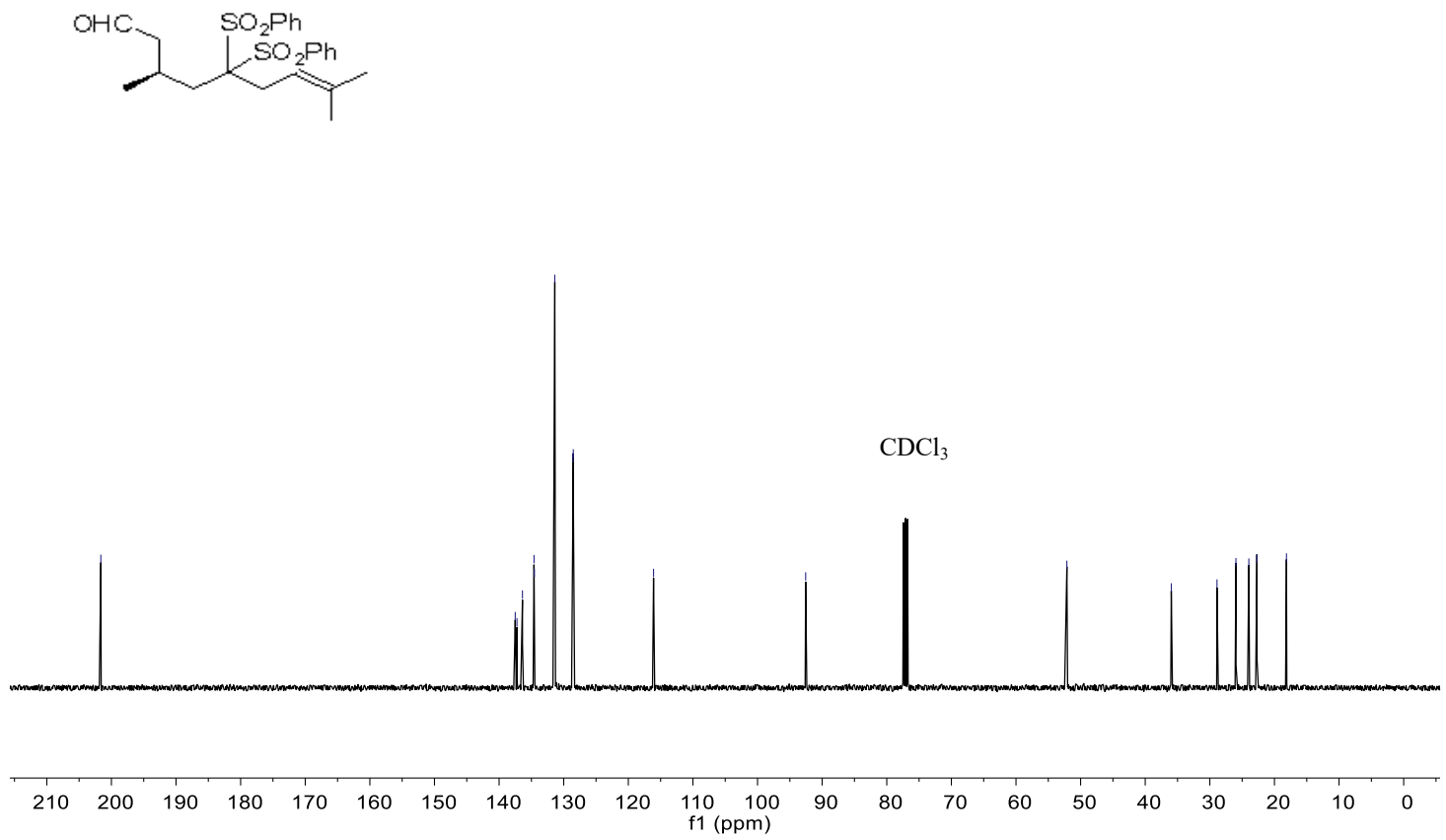

S57 
<smiles>CC(CC=O)CC(C)([Se]Oc1ccccc1)C(=O)Oc1ccccc1</smiles>

${ }^{1} \mathrm{H}$ NMR (400 MHz, $\left.\mathrm{CDCl}_{3}\right)$

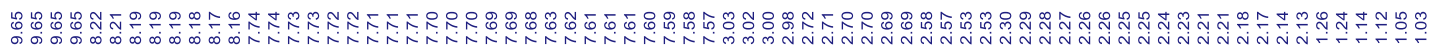
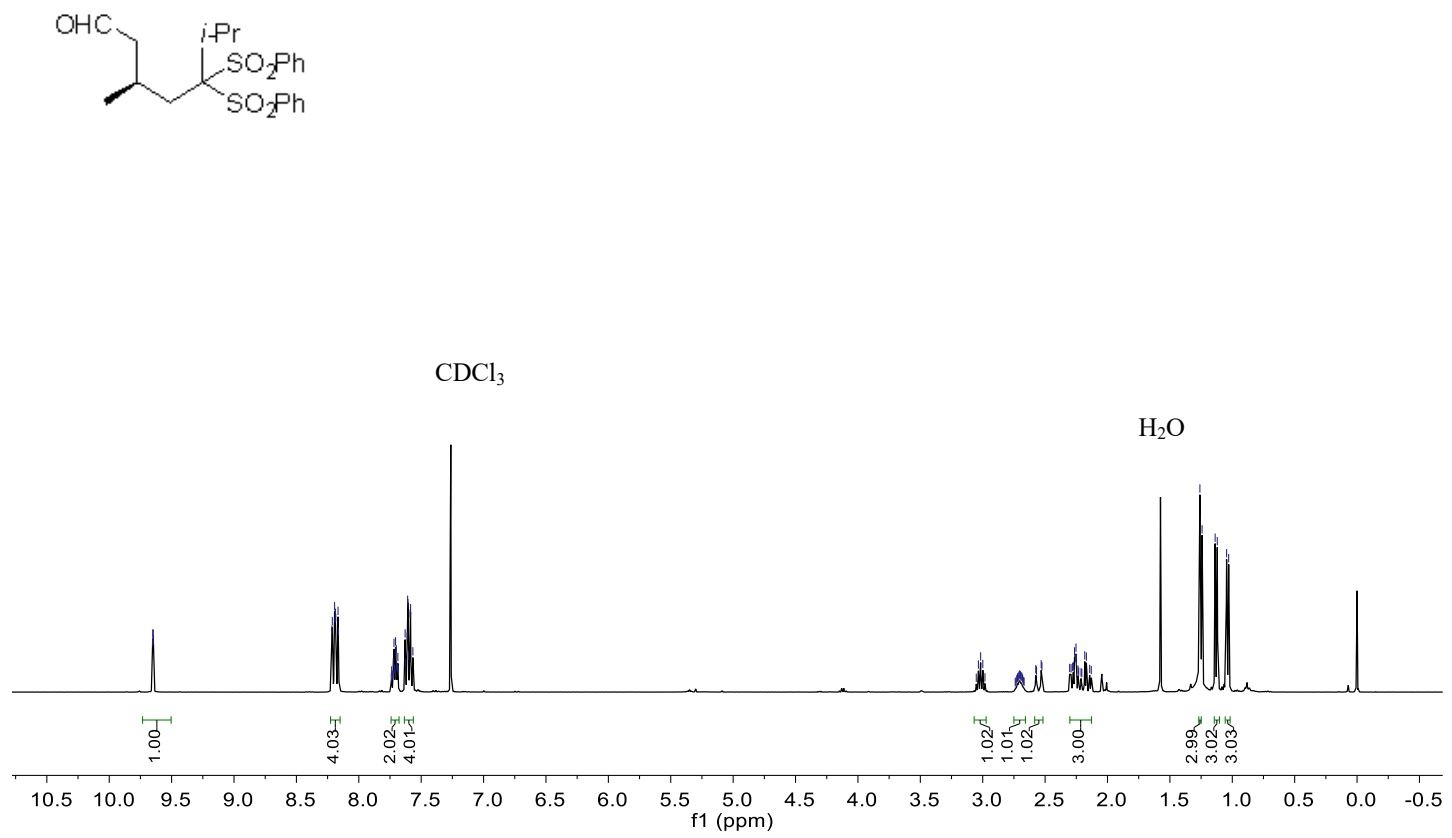

${ }^{13} \mathrm{C}$ NMR (101 MHz, $\left.\mathrm{CDCl}_{3}\right)$

$\stackrel{m}{\stackrel{m}{n}}$

$\underbrace{0}$

$\infty$
$\infty$
$\infty$
$\infty$
1

i nj

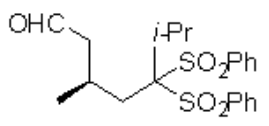

$\mathrm{CDCl}_{3}$
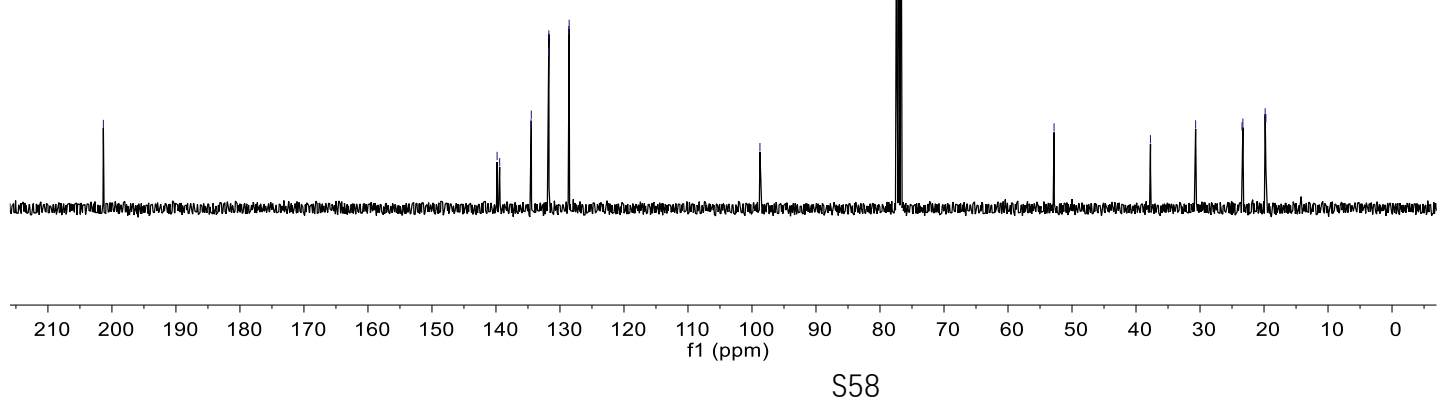
<smiles>CC(CC=O)CC(Br)(O[Pb])[O+]Sc1ccccc1</smiles>

${ }^{1} \mathrm{H}$ NMR (400 MHz, $\left.\mathrm{CDCl}_{3}\right)$

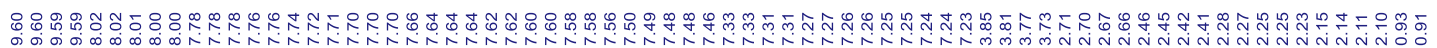
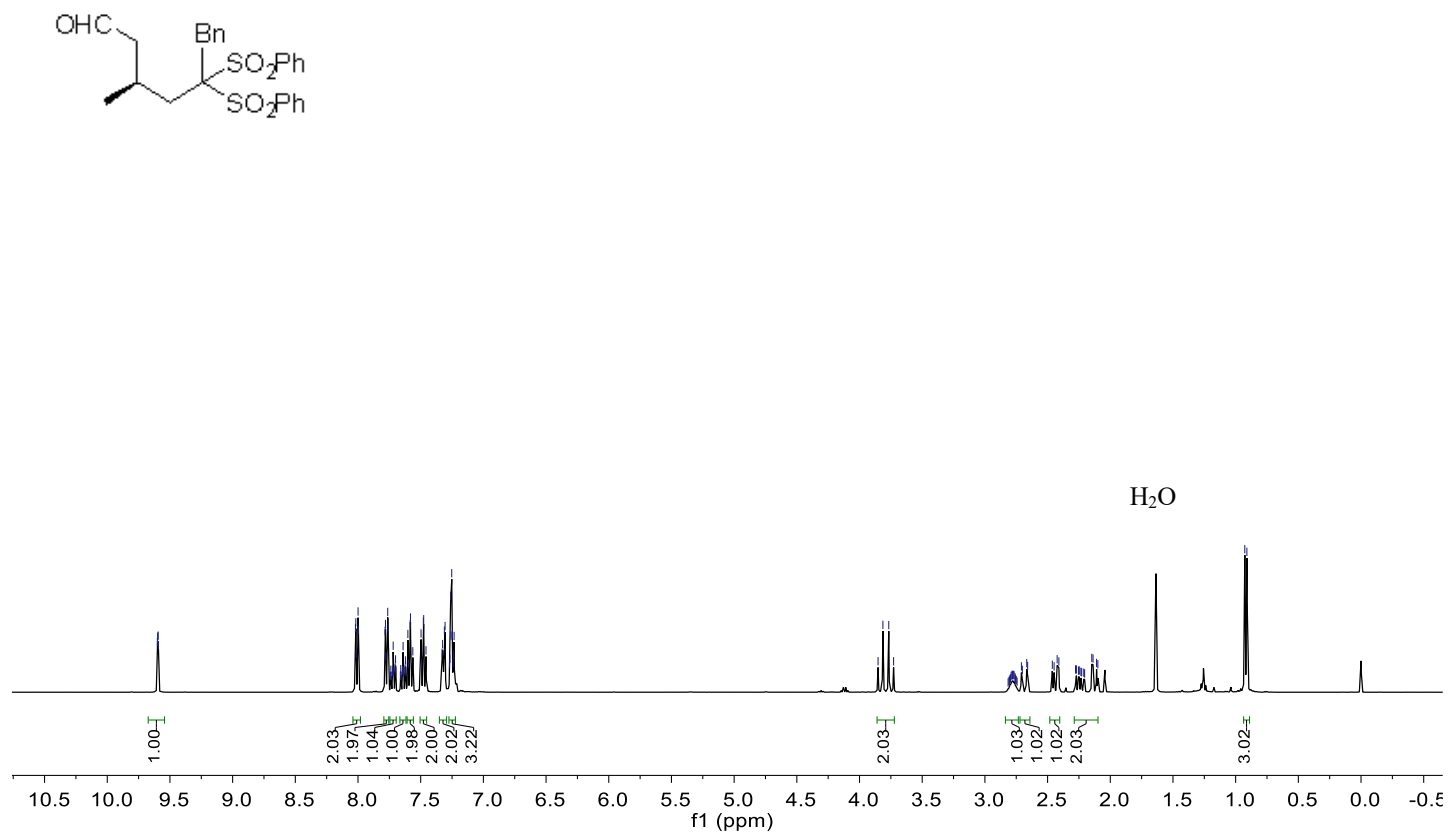

${ }^{13} \mathrm{C}$ NMR (101 MHz, $\left.\mathrm{CDCl}_{3}\right)$

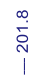

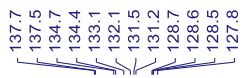

$\underset{\substack{n \\ \infty}}{1}$

in

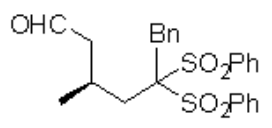

$\mathrm{CDCl}_{3}$
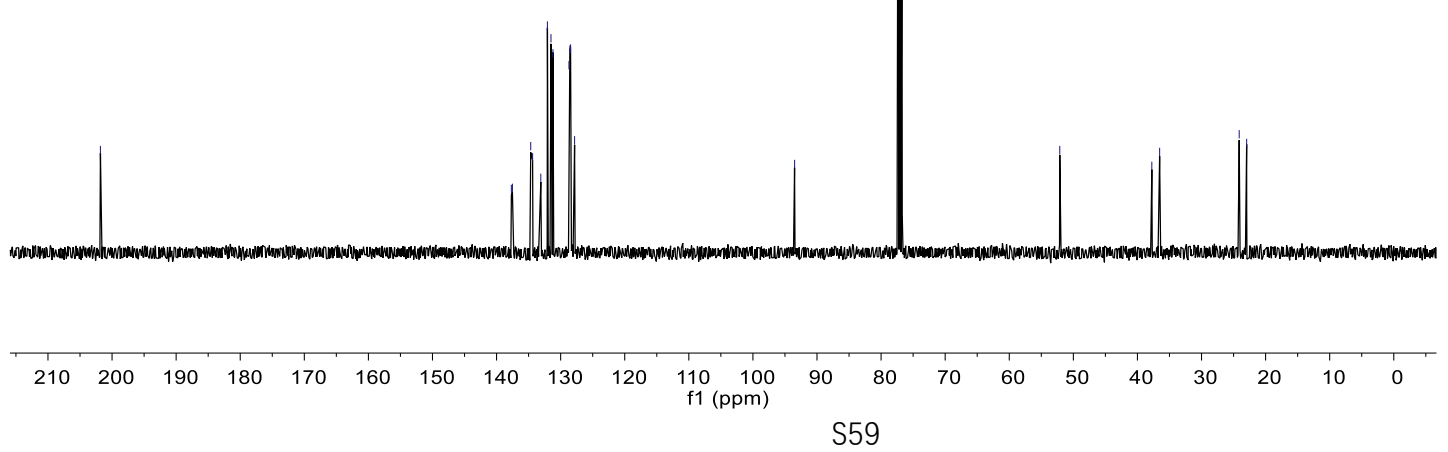


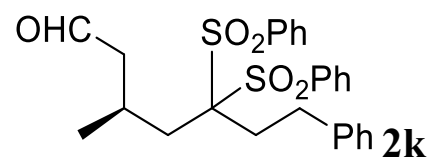

\section{${ }^{1} \mathrm{H}$ NMR (600 MHz, $\left.\mathrm{CDCl}_{3}\right)$}

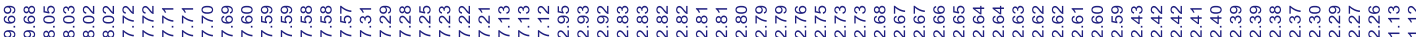

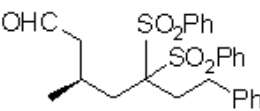

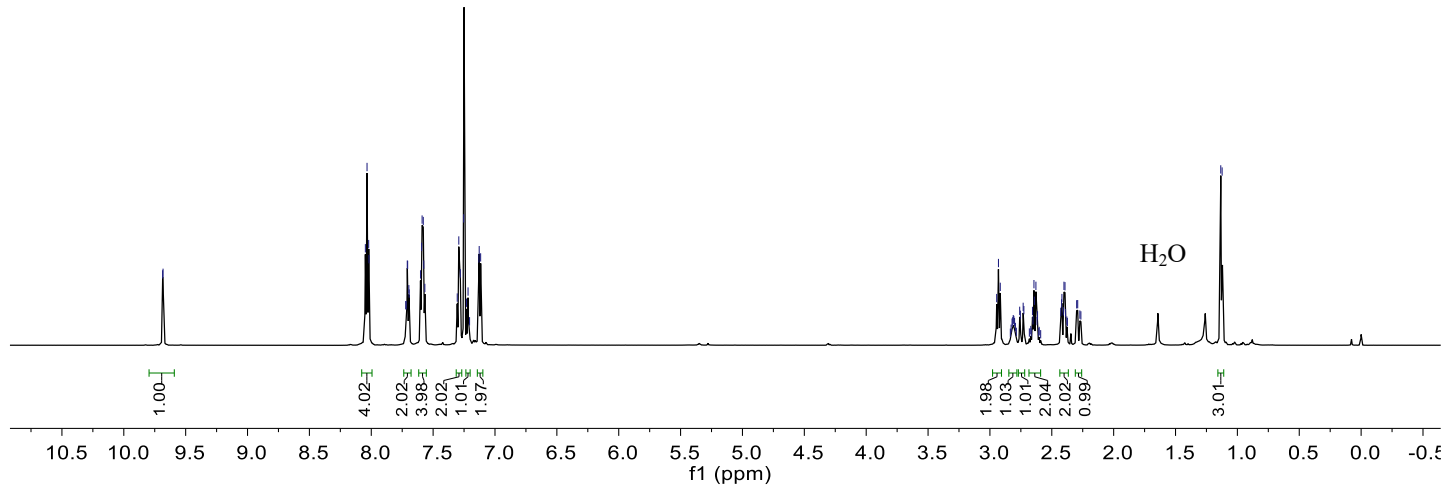

${ }^{13} \mathrm{C}$ NMR (101 MHz, $\left.\mathrm{CDCl}_{3}\right)$
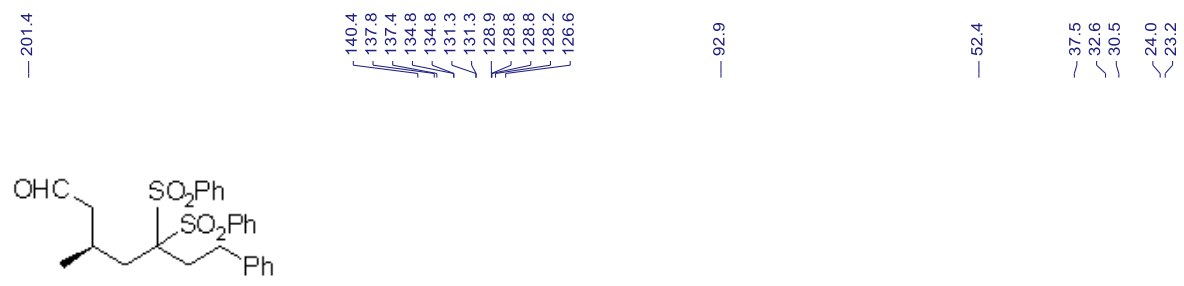

$\mathrm{CDCl}_{3}$

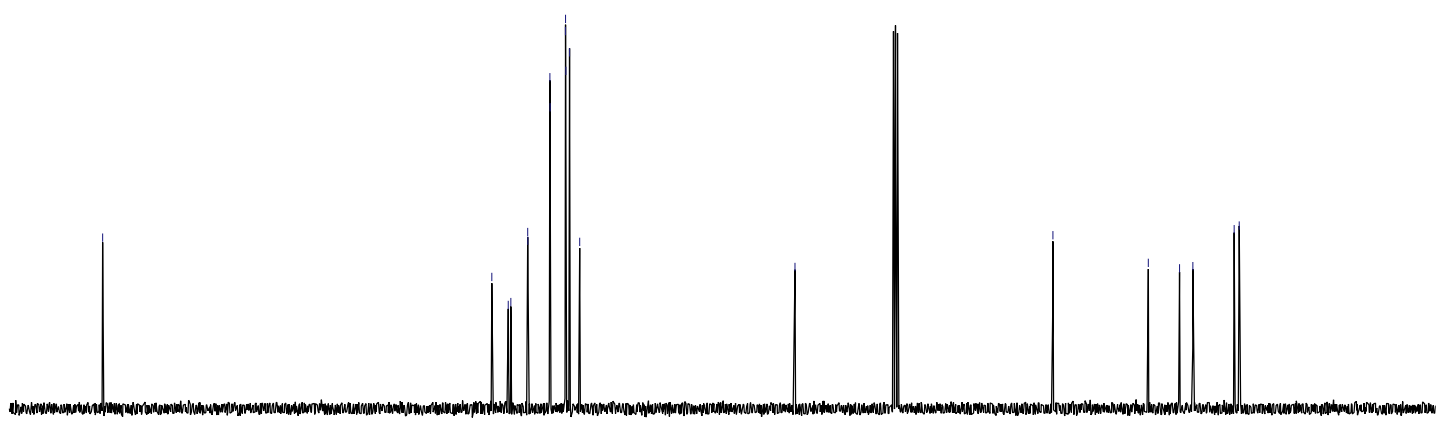

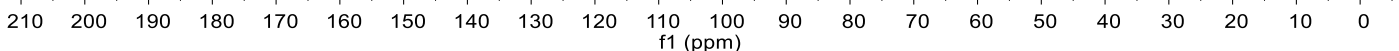




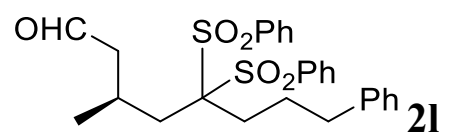

\section{${ }^{1} \mathrm{H}$ NMR (600 MHz, $\left.\mathrm{CDCl}_{3}\right)$}

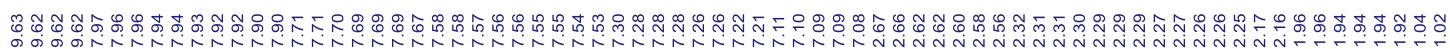

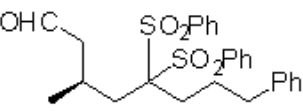

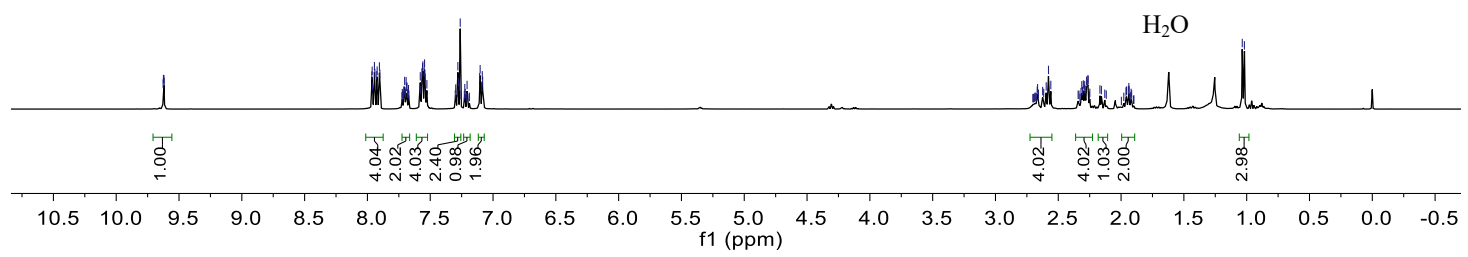

\section{${ }^{13} \mathrm{C}$ NMR (101 MHz, $\left.\mathrm{CDCl}_{3}\right)$}
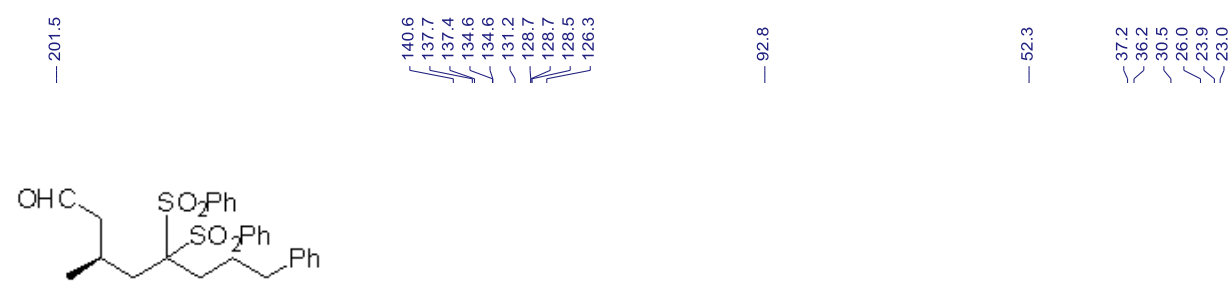

$\mathrm{CDCl}_{3}$

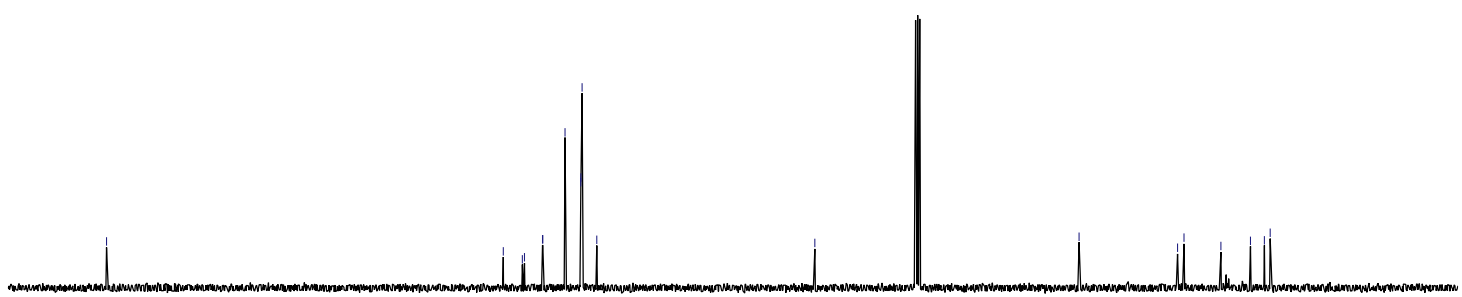

$\begin{array}{lllllllllllllllllllll}210 & 200 & 190 & 180 & 170 & 160 & 150 & 140 & 130 & 120 & \begin{array}{c}110 \\ \mathrm{f} 1(\mathrm{ppm})\end{array} & 90 & 80 & 70 & 60 & 50 & 40 & 30 & 20 & 10 & 0\end{array}$ 


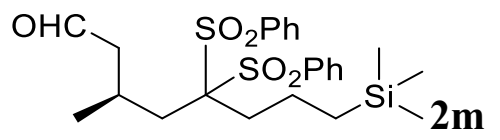

${ }^{1} \mathrm{H}$ NMR (600 MHz, $\left.\mathrm{CDCl}_{3}\right)$

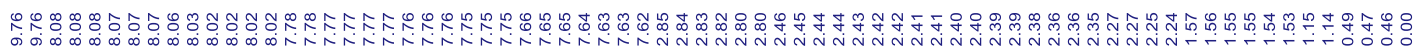

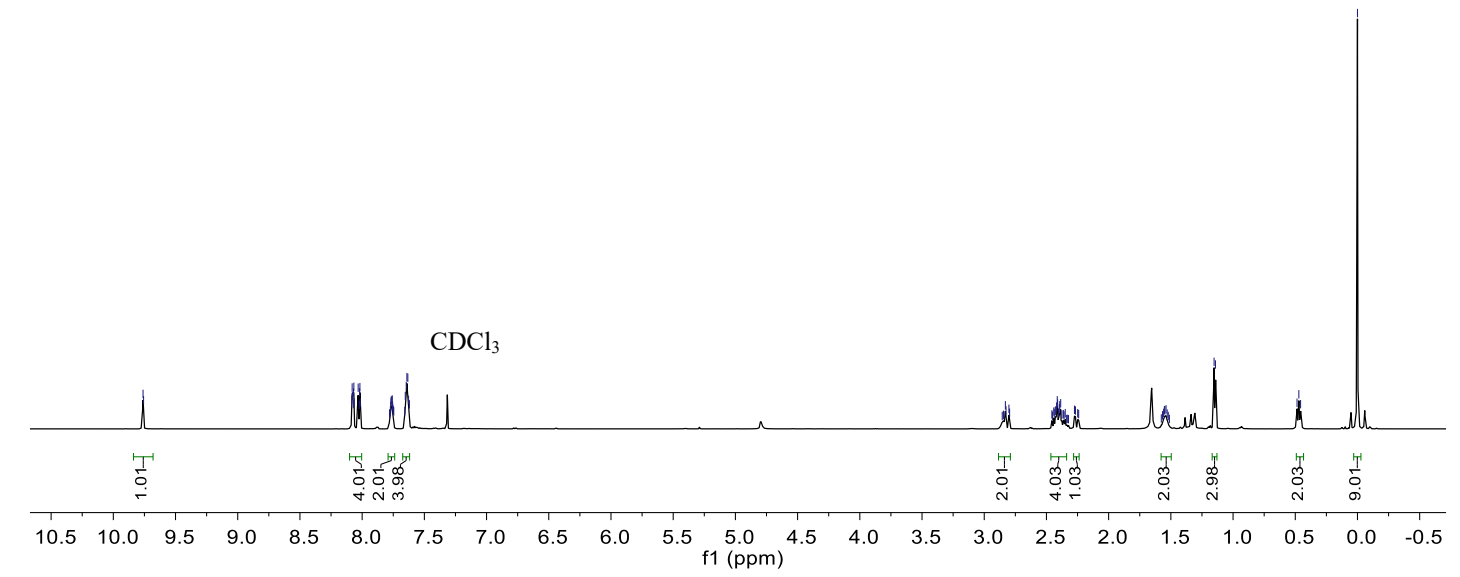

${ }^{13} \mathrm{C}$ NMR (151 MHz, $\left.\mathrm{CDCl}_{3}\right)$

我

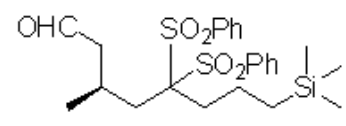

$\mathrm{CDCl}_{3}$

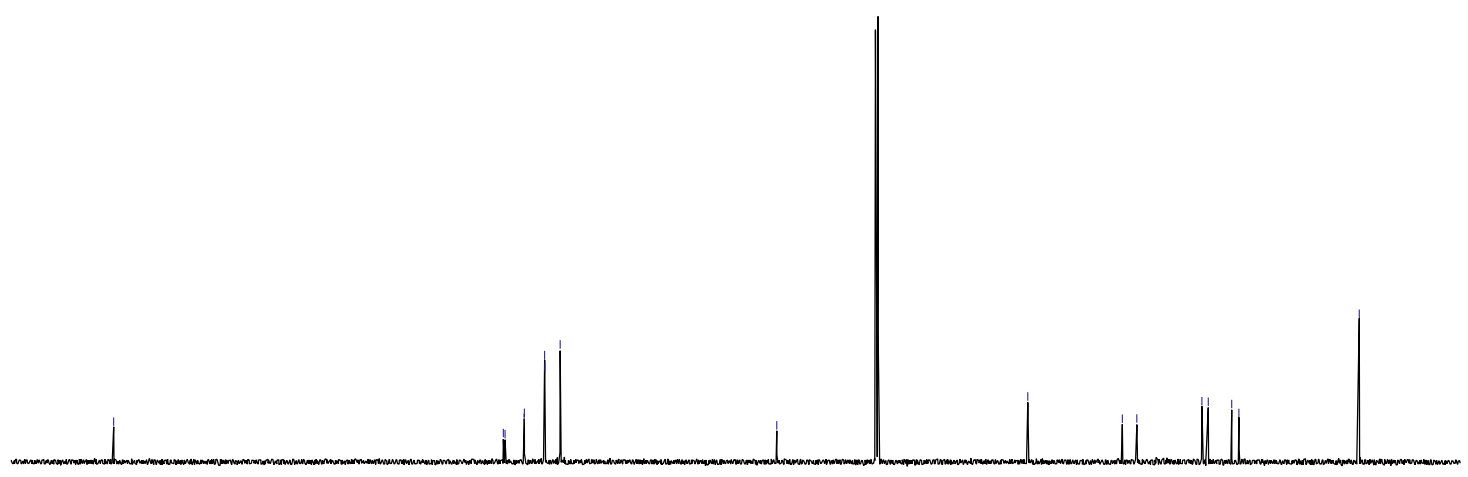

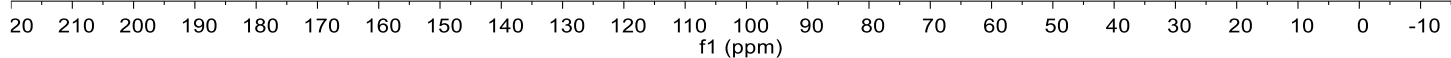




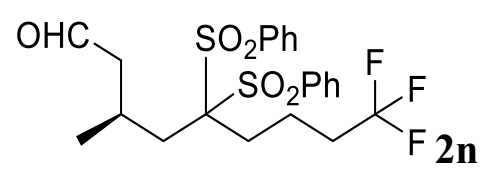

\section{${ }^{1} \mathrm{H}$ NMR (400 MHz, $\left.\mathrm{CDCl}_{3}\right)$}

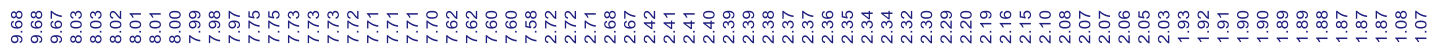

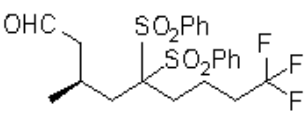

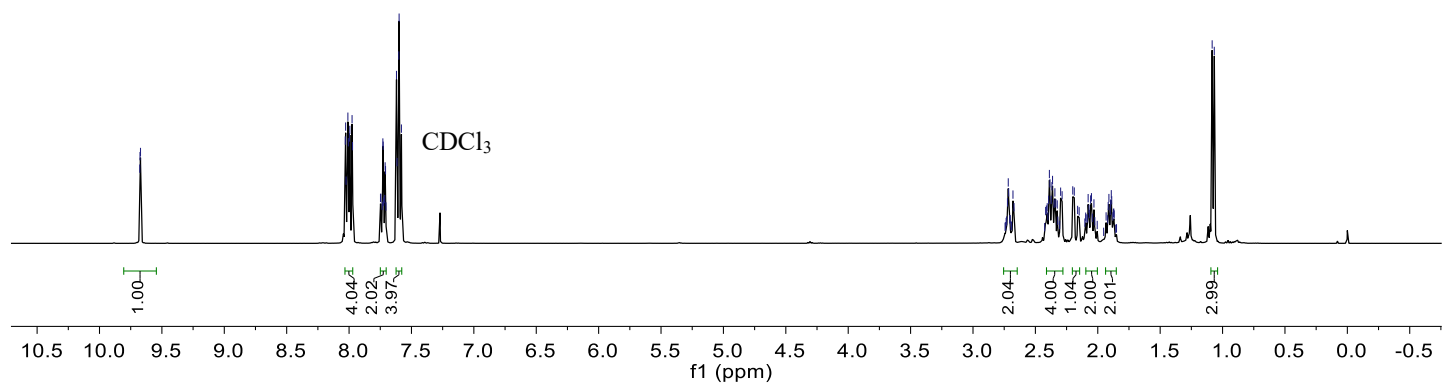

${ }^{13} \mathrm{C}$ NMR (151 MHz, CDCl 3$)$

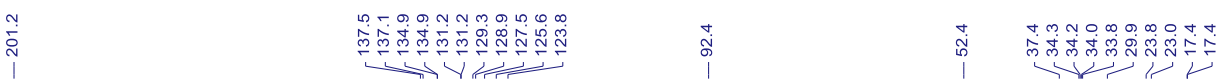

$\underbrace{\mathrm{OHC}} \underbrace{\mathrm{SO}_{2} \mathrm{Ph}}_{\mathrm{F}}$

$\mathrm{CDCl}_{3}$

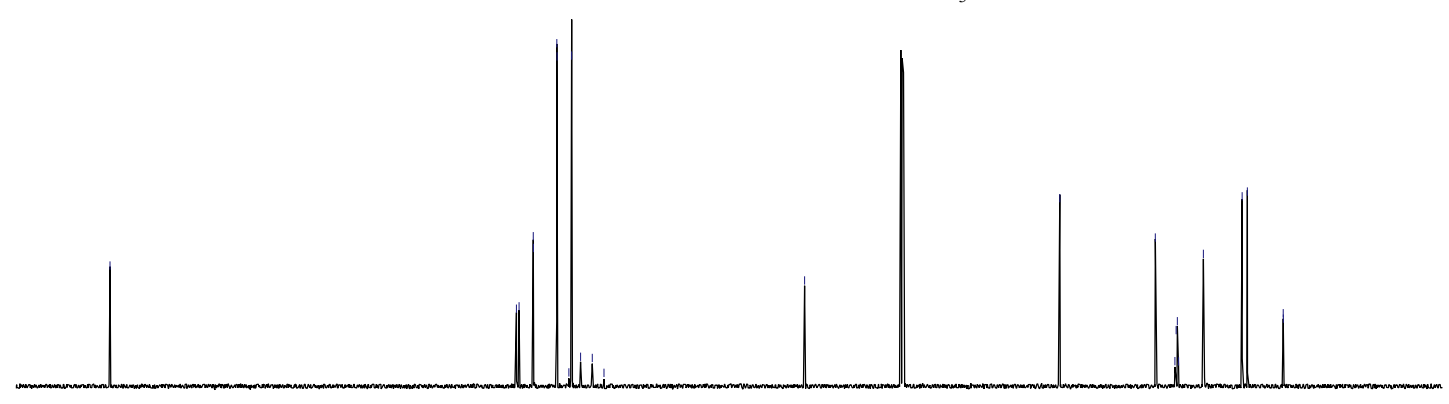

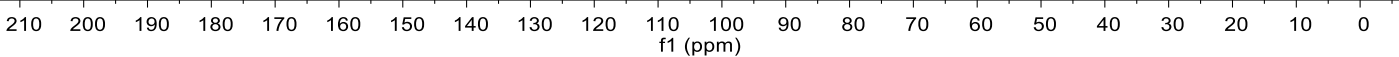


${ }^{19}$ F NMR (565 MHz, $\left.\mathrm{CDCl}_{3}\right)$

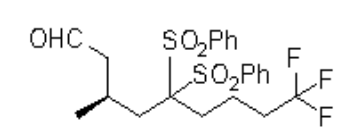

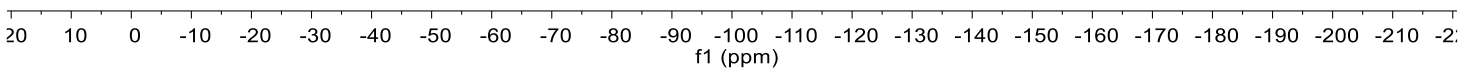

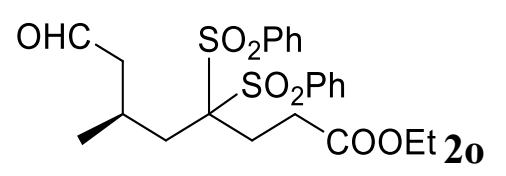

S64 
${ }^{1}$ H NMR (400 MHz, $\left.\mathrm{CDCl}_{3}\right)$

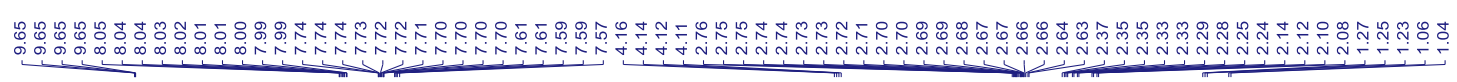
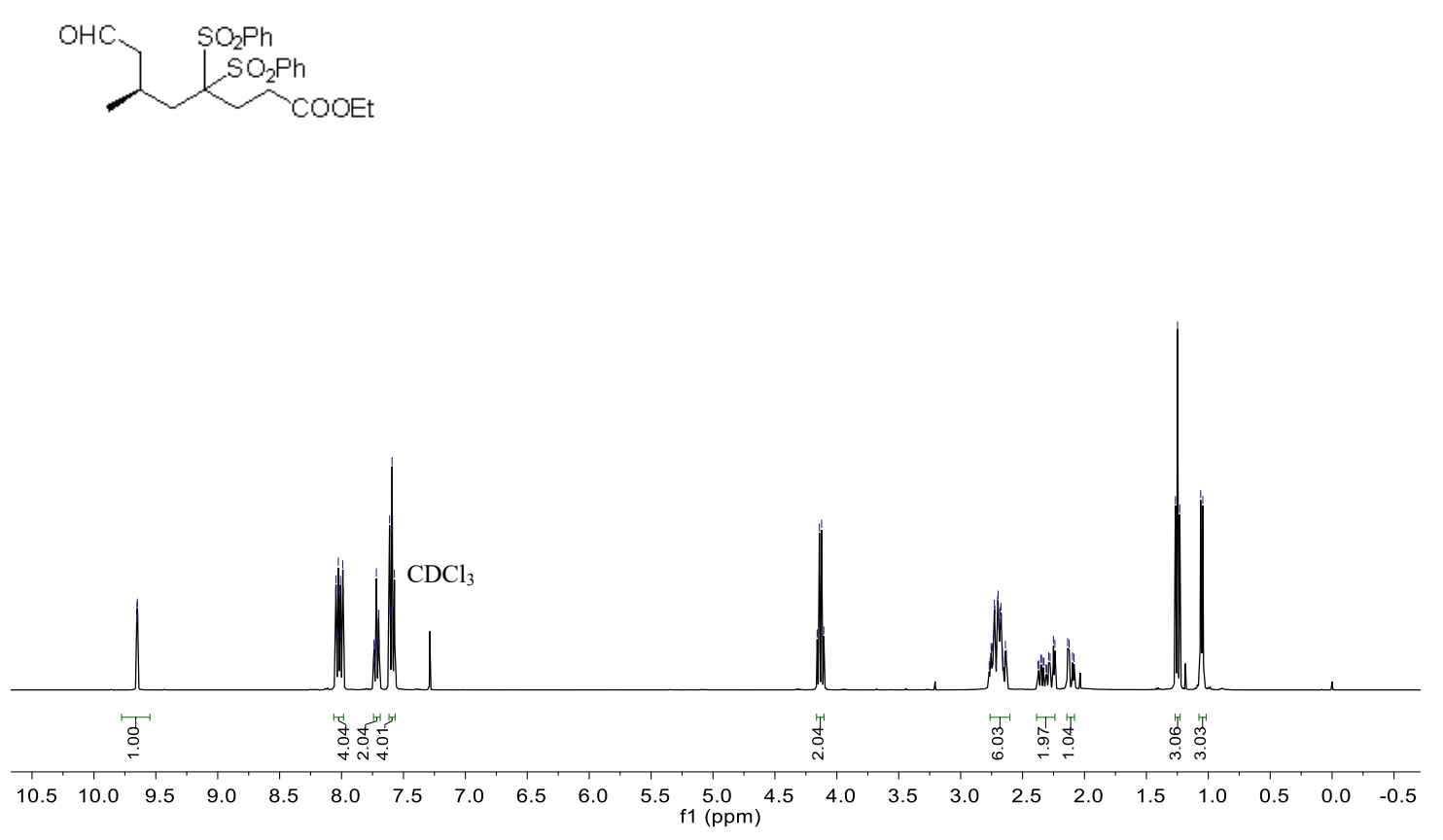

${ }^{13} \mathrm{C}$ NMR (101 MHz, $\left.\mathrm{CDCl}_{3}\right)$
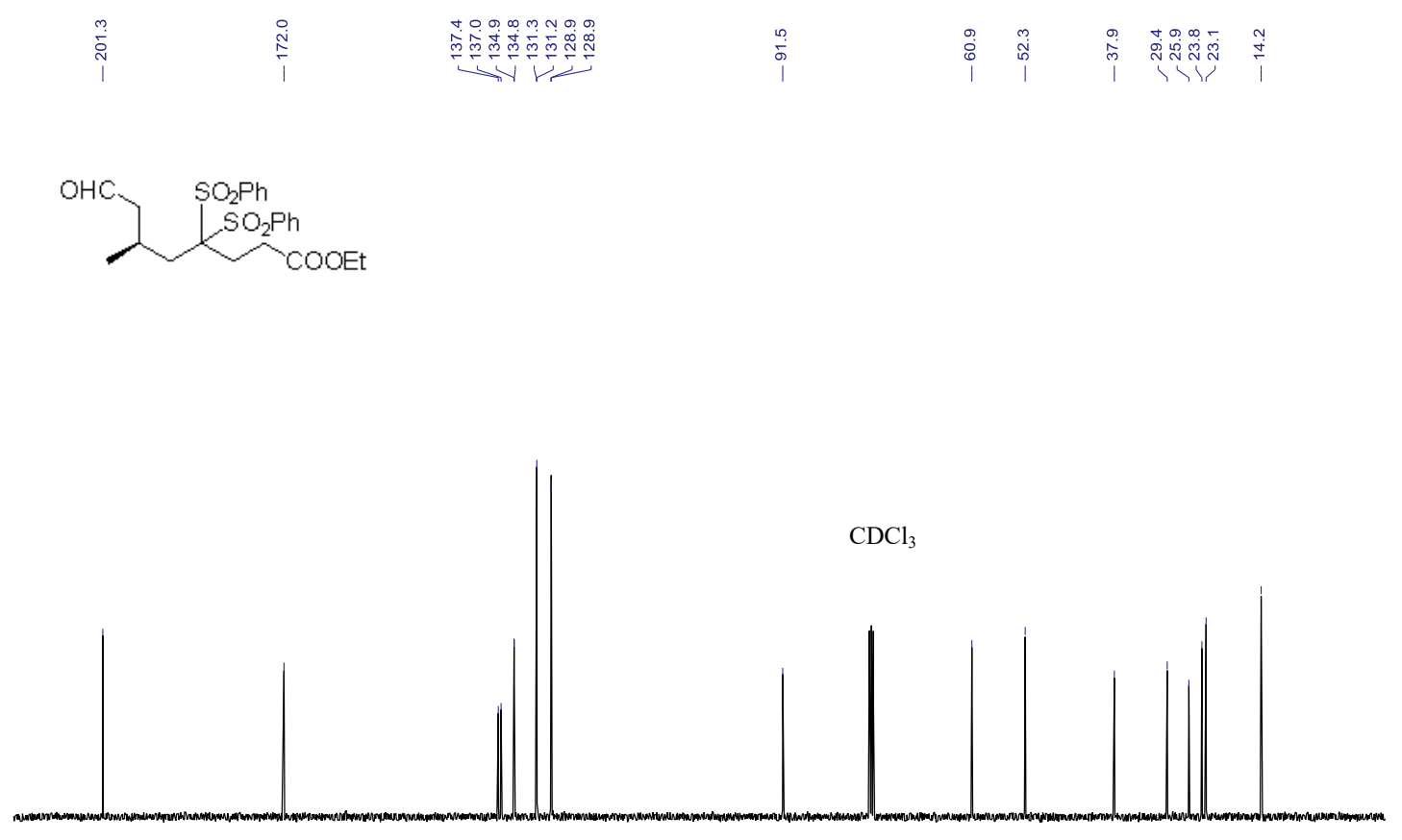

\begin{tabular}{llllllllllllllllllllllllll}
\hline 210 & 200 & 190 & 180 & 170 & 160 & 150 & 140 & 130 & 120 & $\begin{array}{c}110 \\
\mathrm{f} 1(\mathrm{ppm})\end{array}$ & 100 & 80 & 70 & 60 & 50 & 40 & 30 & 20 & 10 & 0 \\
\hline
\end{tabular}

S65 


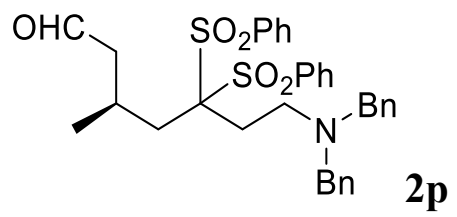

${ }^{1} \mathrm{H}$ NMR (400 MHz, $\left.\mathrm{CDCl}_{3}\right)$

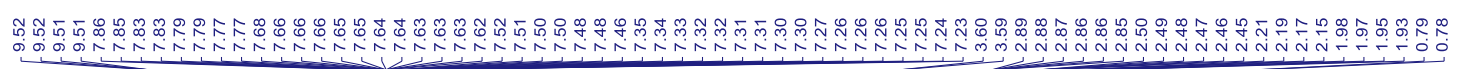
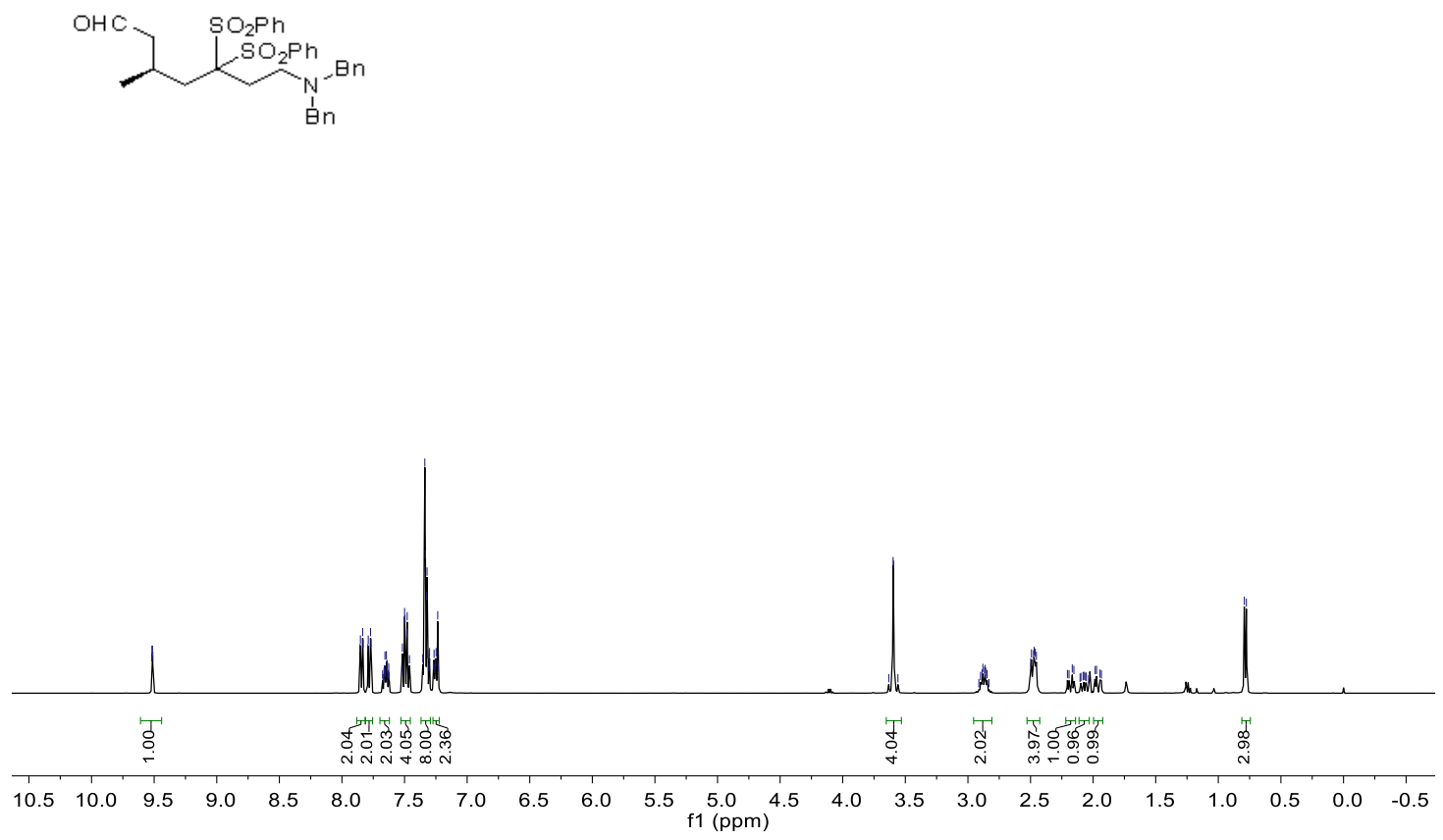

${ }^{13} \mathrm{C}$ NMR (101 MHz, $\left.\mathrm{CDCl}_{3}\right)$

in

$\underbrace{n}$

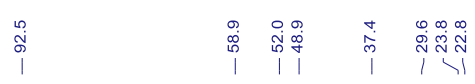
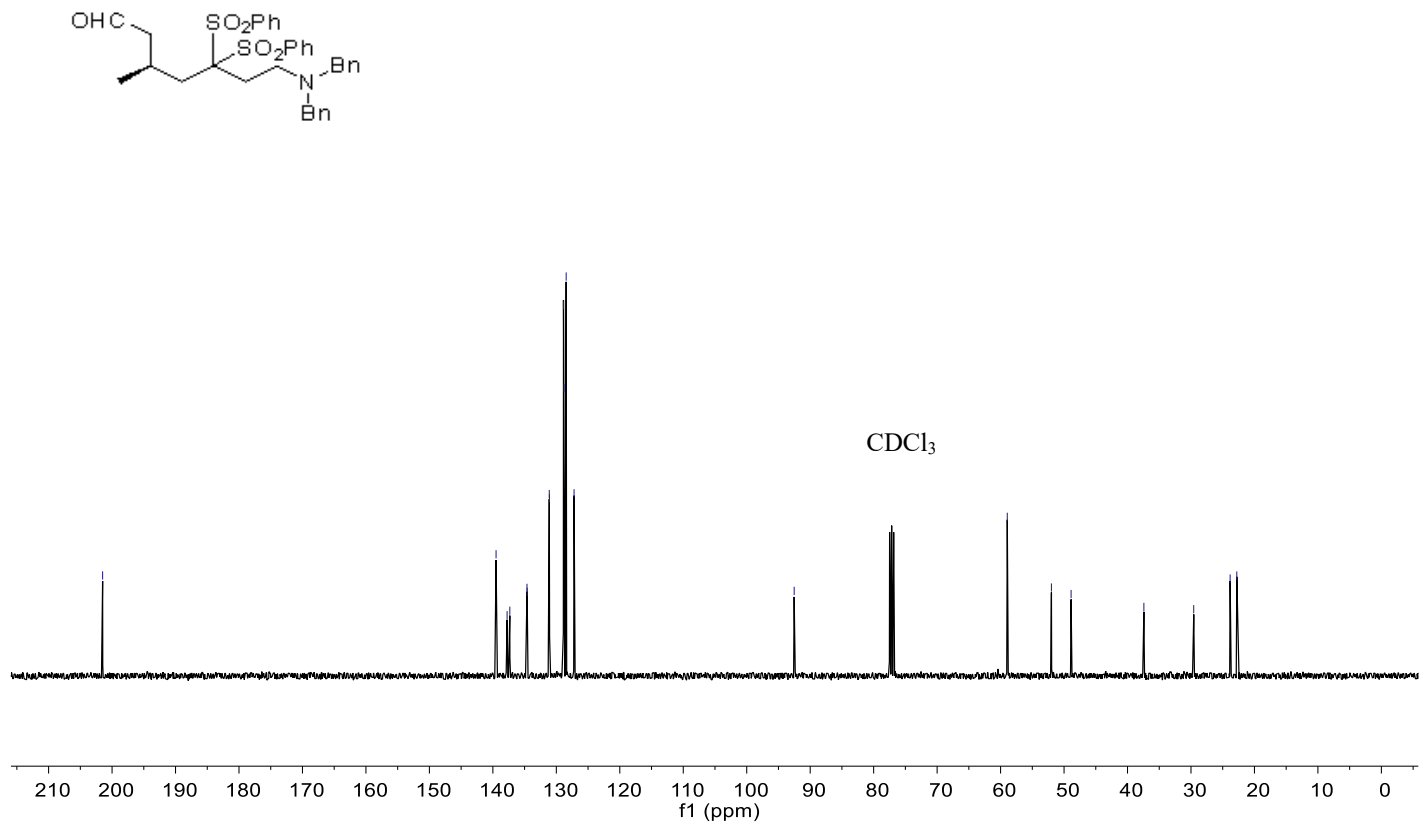

S66 


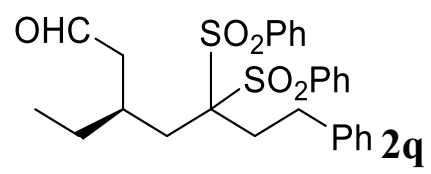

\section{${ }^{1} \mathrm{H}$ NMR (600 MHz, $\left.\mathrm{CDCl}_{3}\right)$}

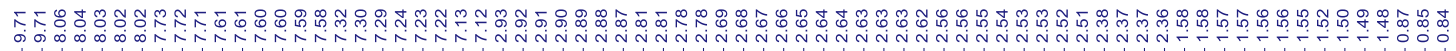<smiles>CC[C@H](CC=O)CC(CC([O+])(c1ccccc1)c1ccccc1)(SO)c1ccccc1</smiles>

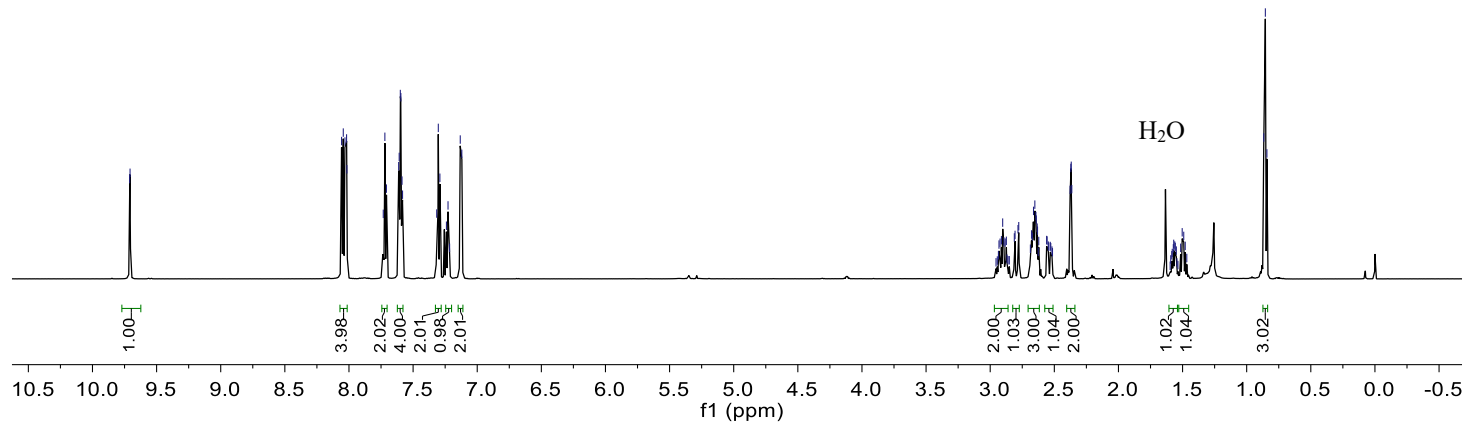

${ }^{13} \mathrm{C}$ NMR (101 MHz, CDCl 3$)$<smiles>[C+]=[C-]C</smiles>

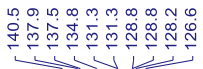<smiles>CCC(CC=O)CC(C)([Se])CC(c1ccccc1)(c1ccccc1)c1ccccc1</smiles>

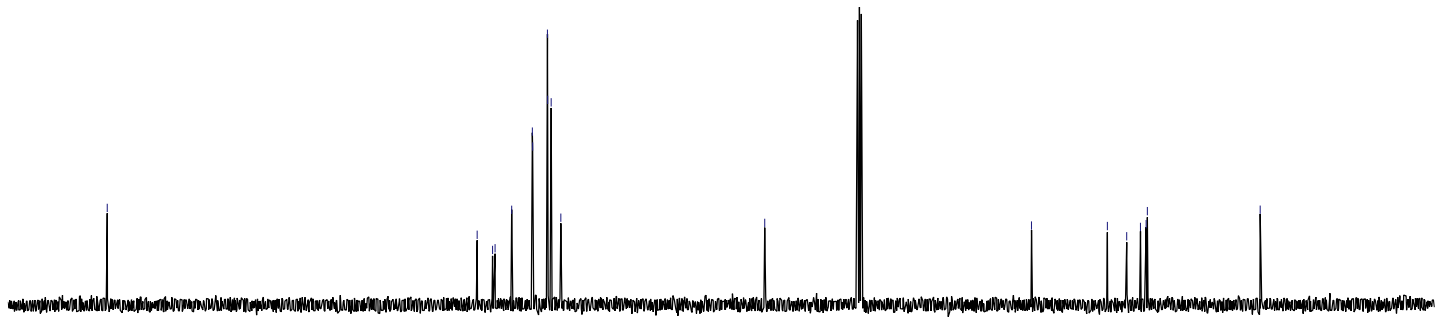




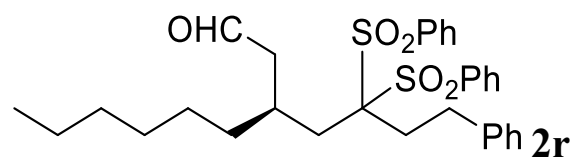

\section{${ }^{1} \mathrm{H}$ NMR (400 MHz, $\left.\mathrm{CDCl}_{3}\right)$}

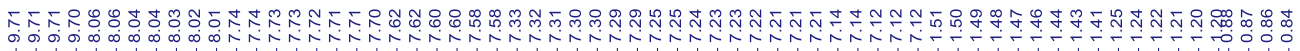
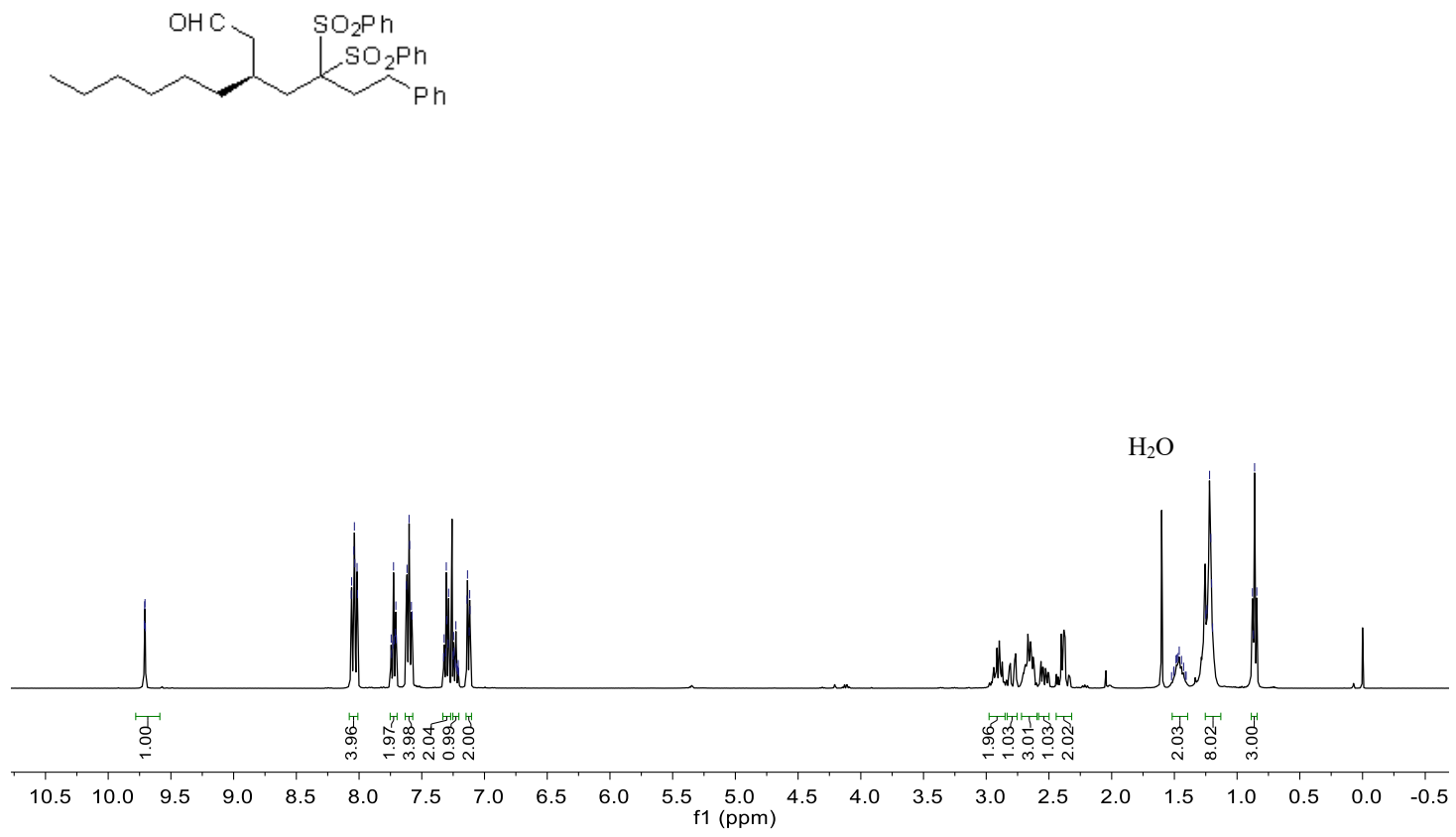

\section{${ }^{13} \mathrm{C}$ NMR (101 MHz, CDCl 3$)$}
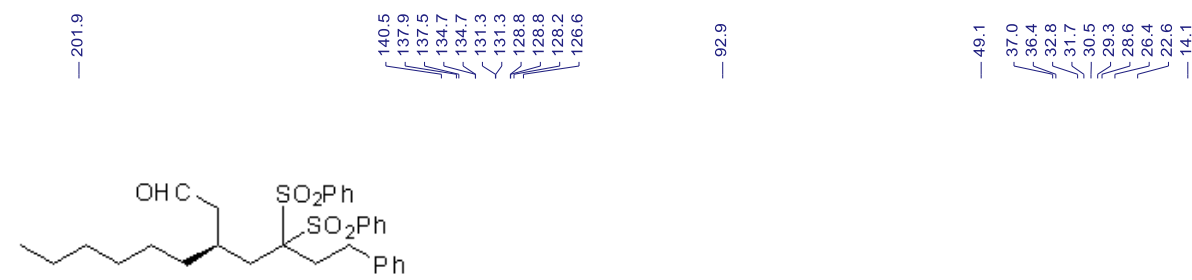

$\mathrm{CDCl}_{3}$ 
$\mathrm{OHC} \quad \mathrm{SO}_{2} \mathrm{Ph}$

$\overbrace{\mathrm{O}^{-} \mathrm{Ph}_{2 \mathrm{~S}}}^{\mathrm{SO}_{2} \mathrm{Ph}}$

${ }^{1} \mathrm{H}$ NMR (400 MHz, $\left.\mathrm{CDCl}_{3}\right)$

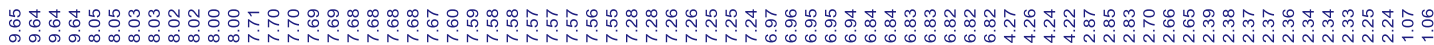

$\mathrm{OHC}, \mathrm{SO}_{2} \mathrm{Ph}$

$\lambda \underbrace{\mathrm{SO}_{2} \mathrm{Ph}}_{0^{-} \mathrm{Ph}}$

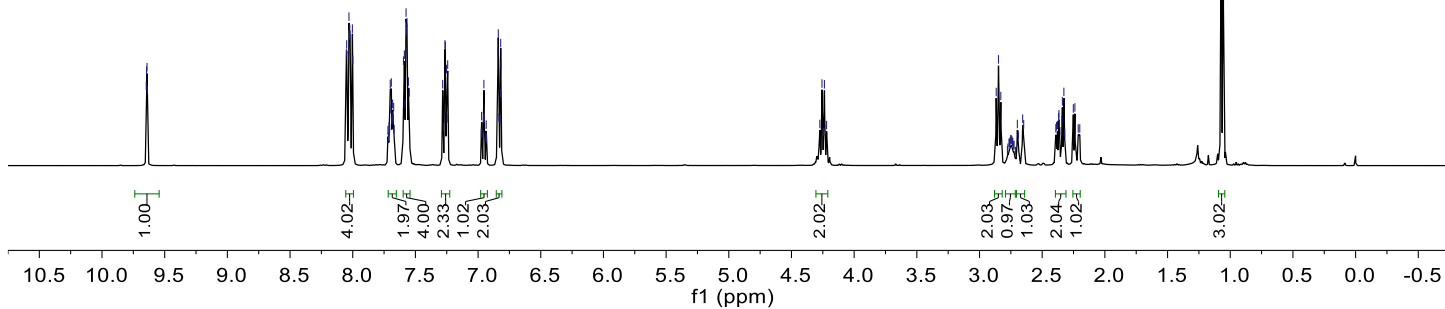

${ }^{13} \mathrm{C}$ NMR (101 MHz, $\left.\mathrm{CDCl}_{3}\right)$

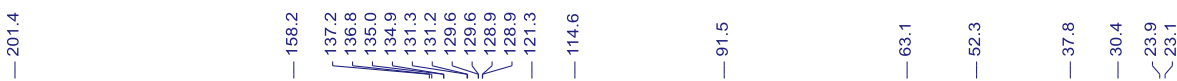

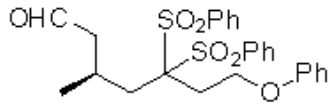

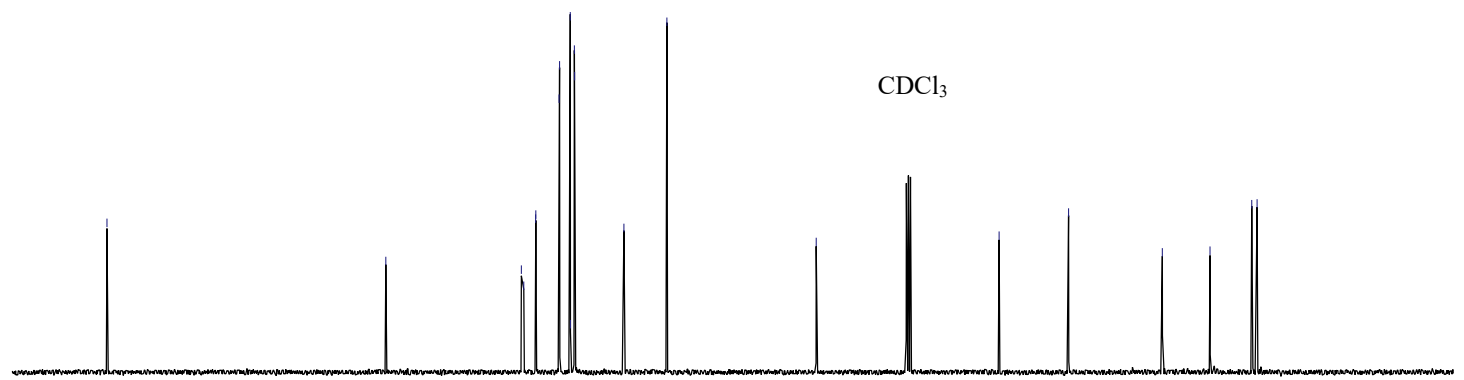

$\begin{array}{llllllllllllllllllllll}210 & 200 & 190 & 180 & 170 & 160 & 150 & 140 & 130 & 120 & \begin{array}{c}110 \\ \mathrm{f} 1(\mathrm{ppm})\end{array} & \begin{array}{l}100 \\ 90\end{array} & 80 & 70 & 60 & 50 & 40 & 30 & 20 & 10 & 0\end{array}$ 
$\mathrm{OHC} \quad \mathrm{SO}_{2} \mathrm{Ph}$

$\overbrace{\mathrm{S}^{-} \mathrm{Ph}}^{\mathrm{SO}_{2} \mathrm{Ph}}$

${ }^{1} \mathrm{H}$ NMR (400 MHz, $\left.\mathrm{CDCl}_{3}\right)$

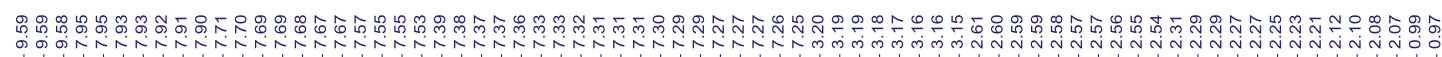

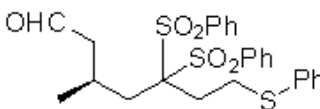

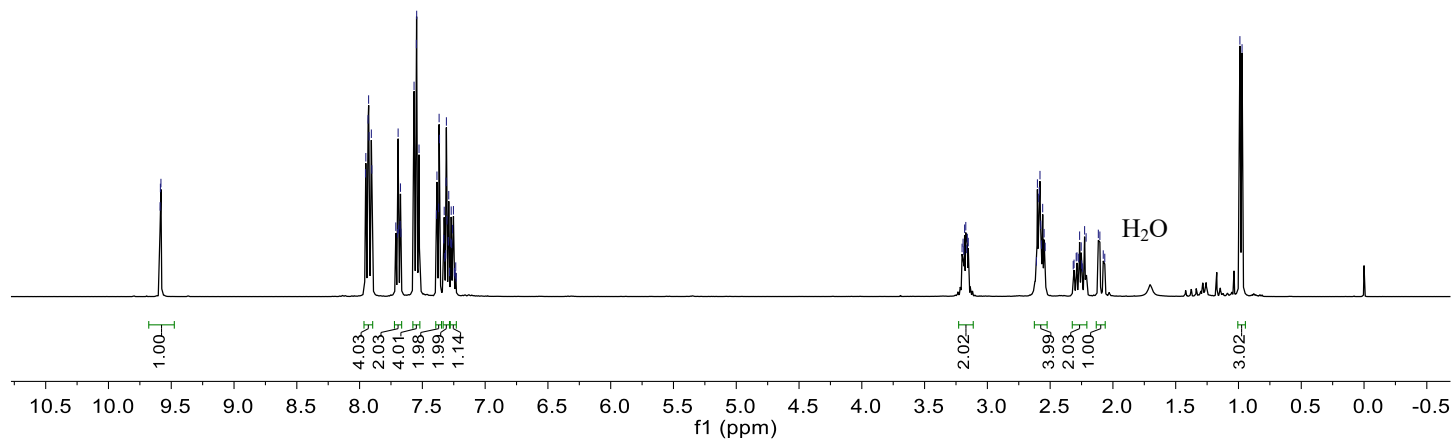

${ }^{13} \mathrm{C}$ NMR (101 MHz, $\left.\mathrm{CDCl}_{3}\right)$

离

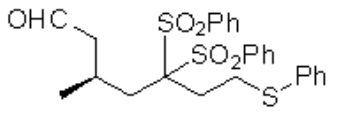

$\mathrm{CDCl}_{3}$

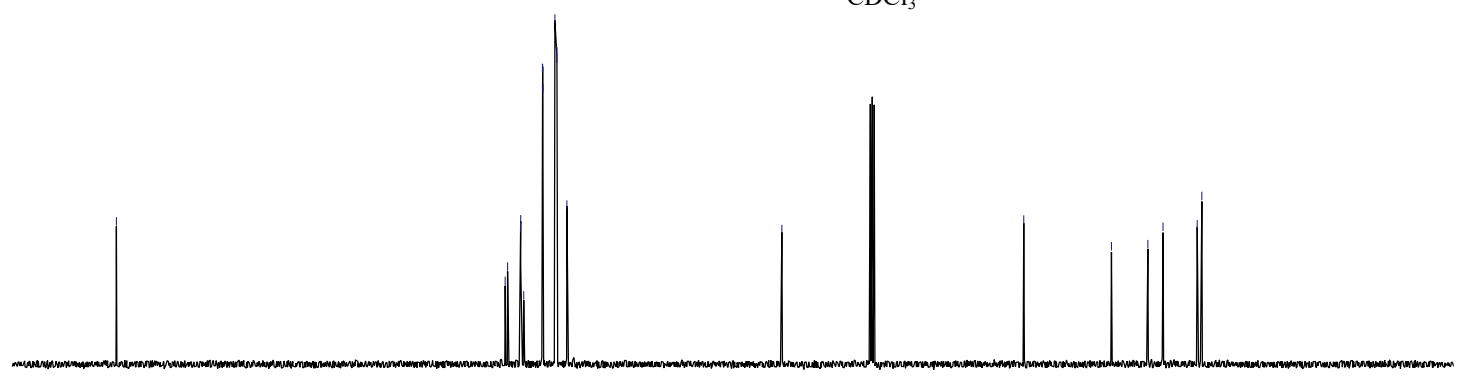

$\begin{array}{lllllllllllllllllllllll}210 & 200 & 190 & 180 & 170 & 160 & 150 & 140 & 130 & 120 & 110 & 100 & 90 & 80 & 70 & 60 & 50 & 40 & 30 & 20 & 10 & 0 & -10\end{array}$ 
$\mathrm{OHC}$<smiles>CC(C)CCCC[PbH2]</smiles>

${ }^{1} \mathrm{H}$ NMR (400 MHz, $\left.\mathrm{CDCl}_{3}\right)$

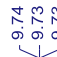

จกำ

inisin

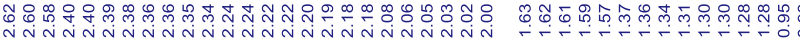

$\mathrm{OHC}$<smiles>CC(C)CCCCc1ccccc1</smiles>

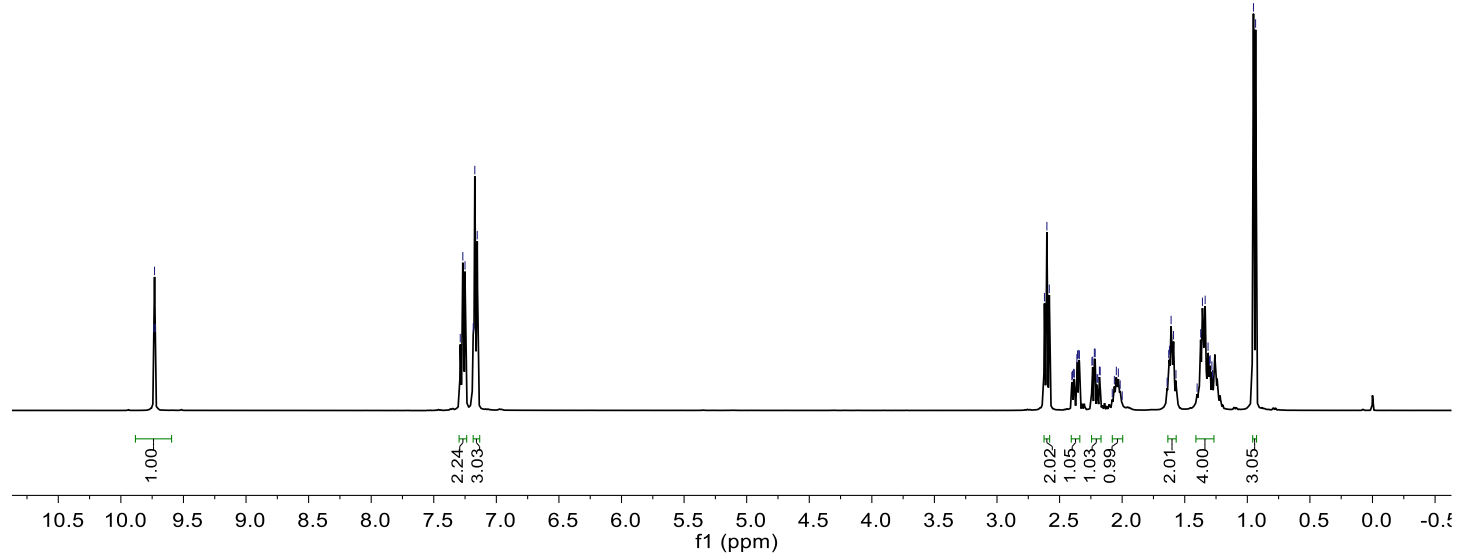

${ }^{13} \mathrm{C}$ NMR (101 MHz, $\left.\mathrm{CDCl}_{3}\right)$
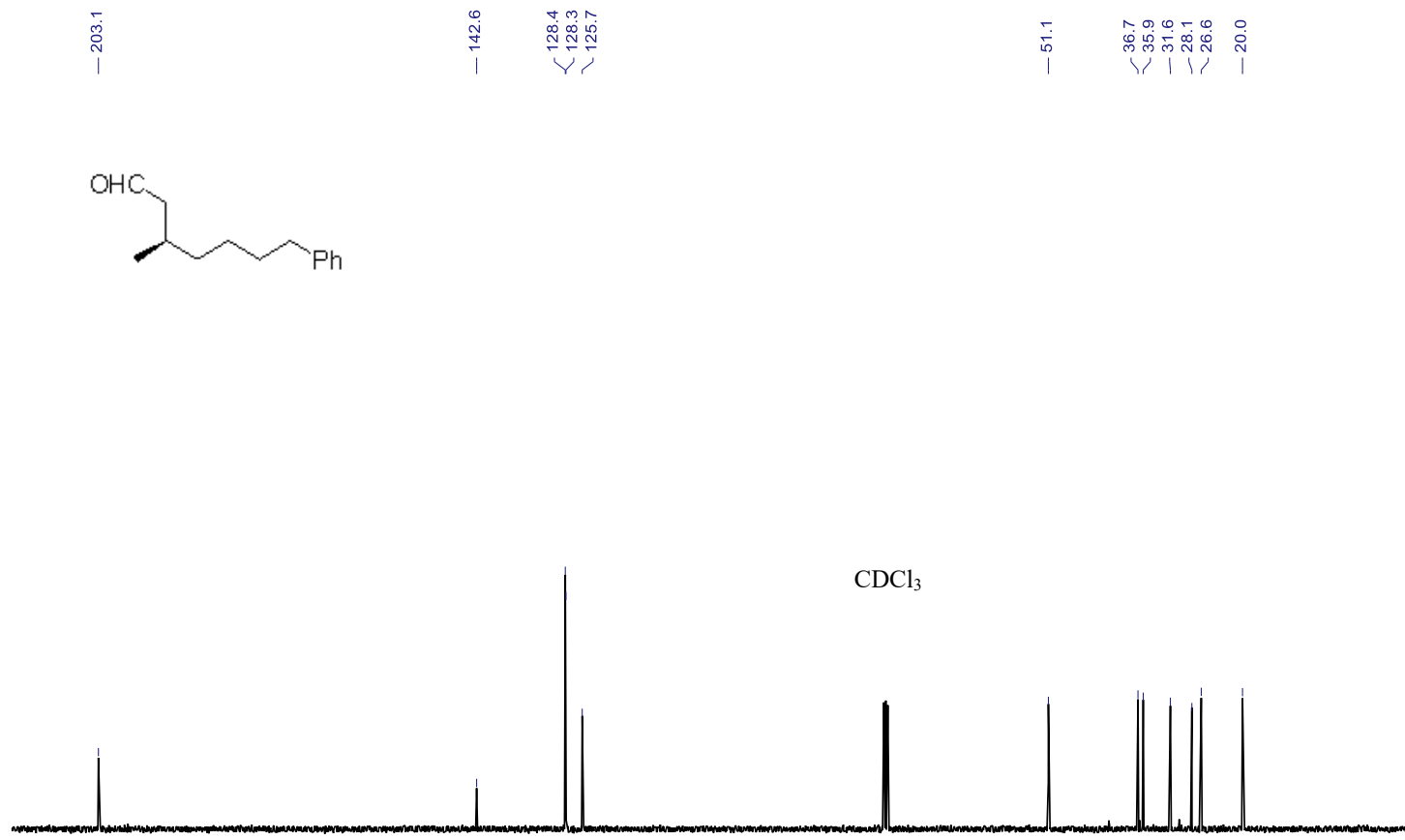

$\begin{array}{lllllllllllllllllllll}210 & 200 & 190 & 180 & 170 & 160 & 150 & 140 & 130 & 120 & \begin{array}{l}110 \\ \mathrm{f} 1(\mathrm{ppm})\end{array} & 100 & 80 & 70 & 60 & 50 & 40 & 30 & 20 & 10 & 0\end{array}$ 


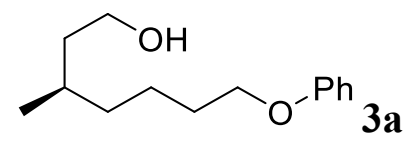

\section{${ }^{1} \mathrm{H}$ NMR (600 MHz, $\left.\mathrm{CDCl}_{3}\right)$}

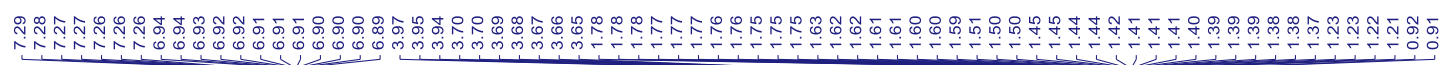<smiles>CC(CCO)CCCCOc1ccccc1</smiles>

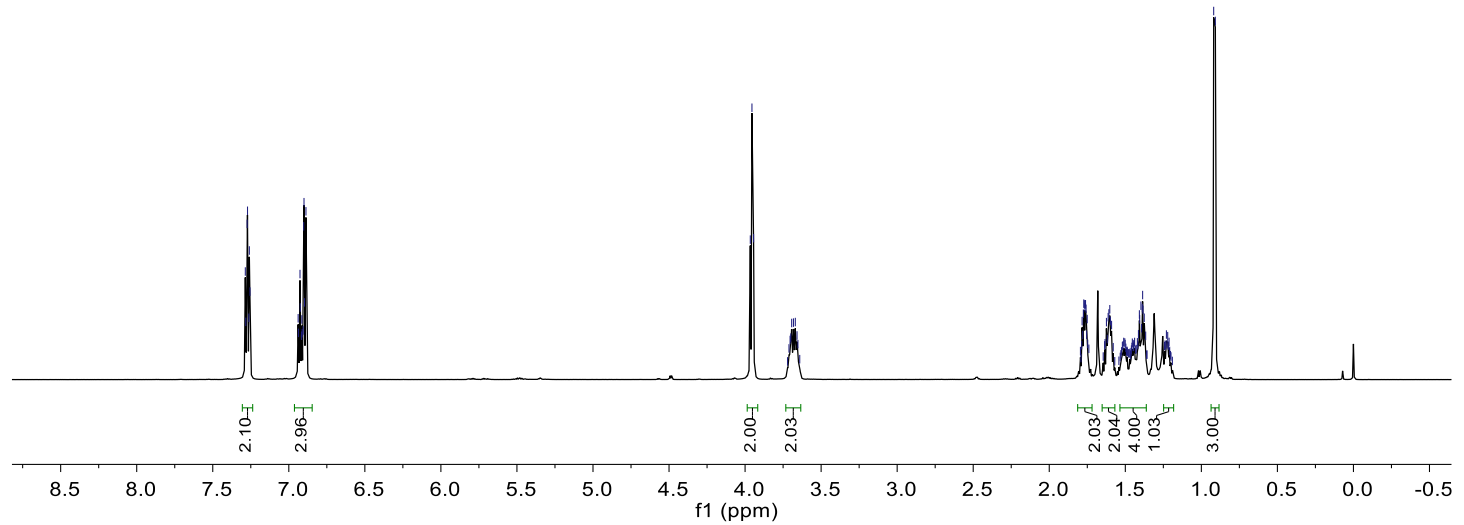

${ }^{13} \mathrm{C}$ NMR (151 MHz, CDCl 3$)$

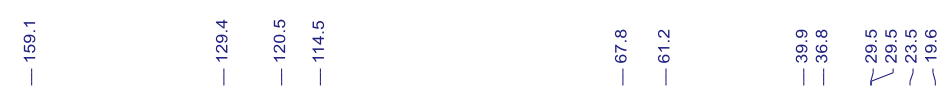<smiles>CC(CCO)CCCCOc1ccccc1</smiles>

$\mathrm{CDCl}_{3}$

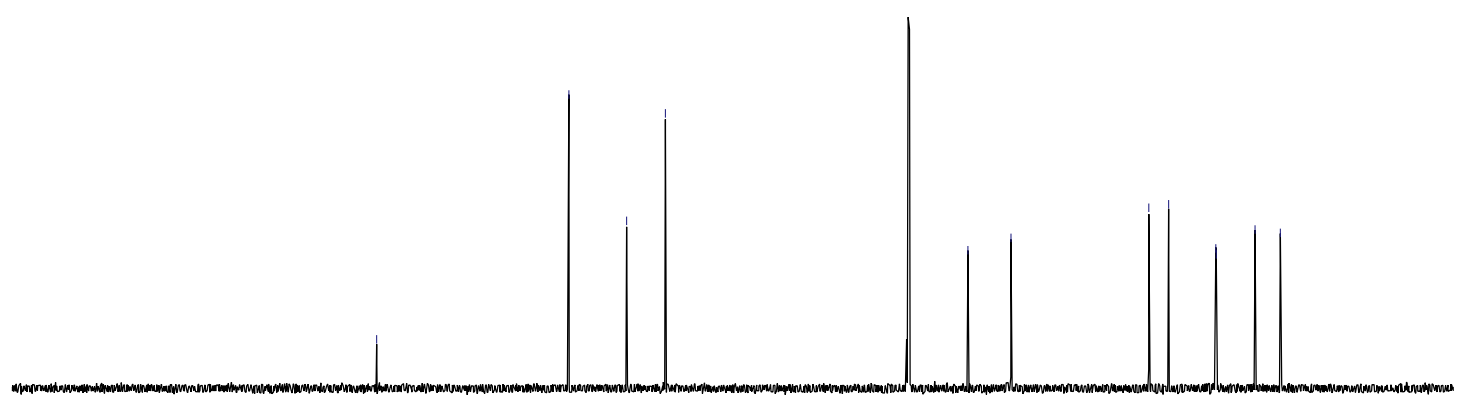

$\begin{array}{rlllllllllllllllllllll}210 & 200 & 190 & 180 & 170 & 160 & 150 & 140 & 130 & 120 & 110 & 100 & 90 & 80 & 70 & 60 & 50 & 40 & 30 & 20 & 10 & 0\end{array}$ 
$\overbrace{\mathrm{O}^{-\mathrm{Ph}}{ }_{3 \mathbf{b}} \mathrm{COOH}}$

${ }^{1} \mathrm{H}$ NMR (400 MHz, $\left.\mathrm{CDCl}_{3}\right)$

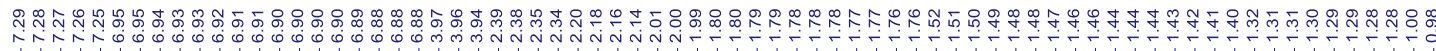<smiles>CC(CCCCOc1ccccc1)CC(=O)O</smiles>

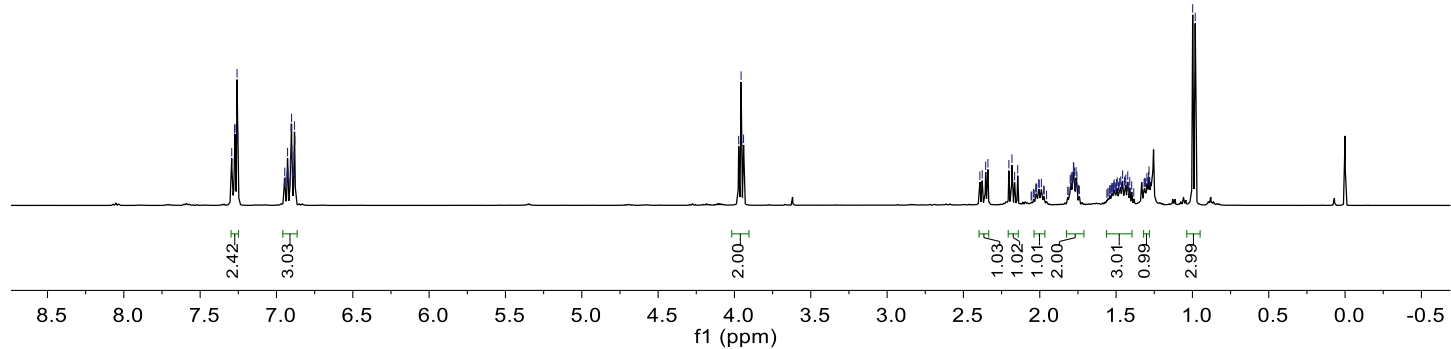

${ }^{13} \mathrm{C}$ NMR (101 MHz, $\left.\mathrm{CDCl}_{3}\right)$

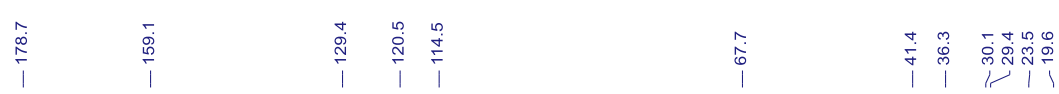<smiles>C[C@H](CCCCOc1ccccc1)CC(=O)O</smiles>

$\mathrm{CDCl}_{3}$

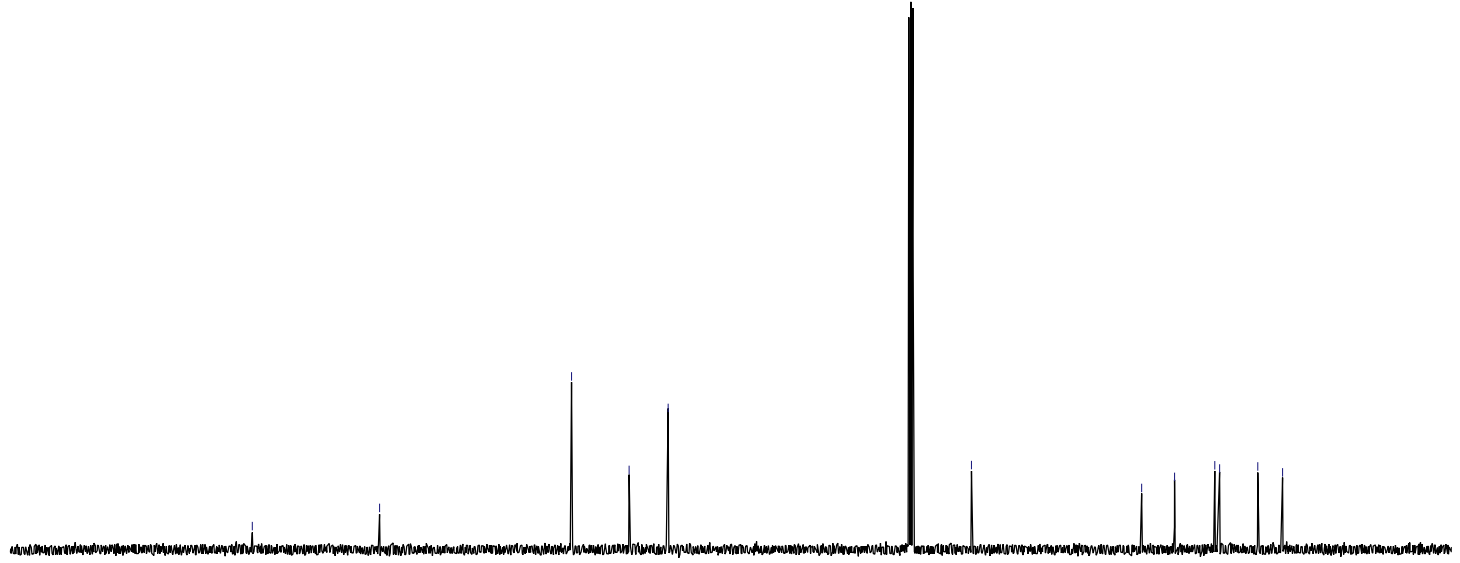

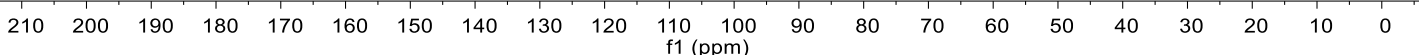




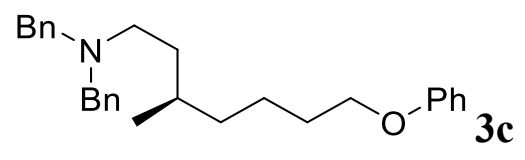

${ }^{1} \mathrm{H}$ NMR (600 MHz, $\left.\mathrm{CDCl}_{3}\right)$

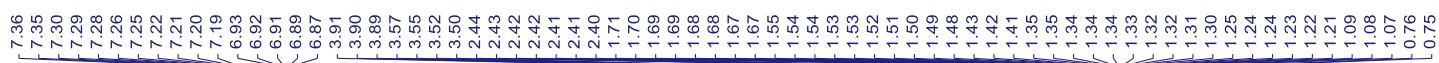<smiles>CC(CCCCOc1ccccc1)CCN(Br)Cc1ccccc1</smiles>

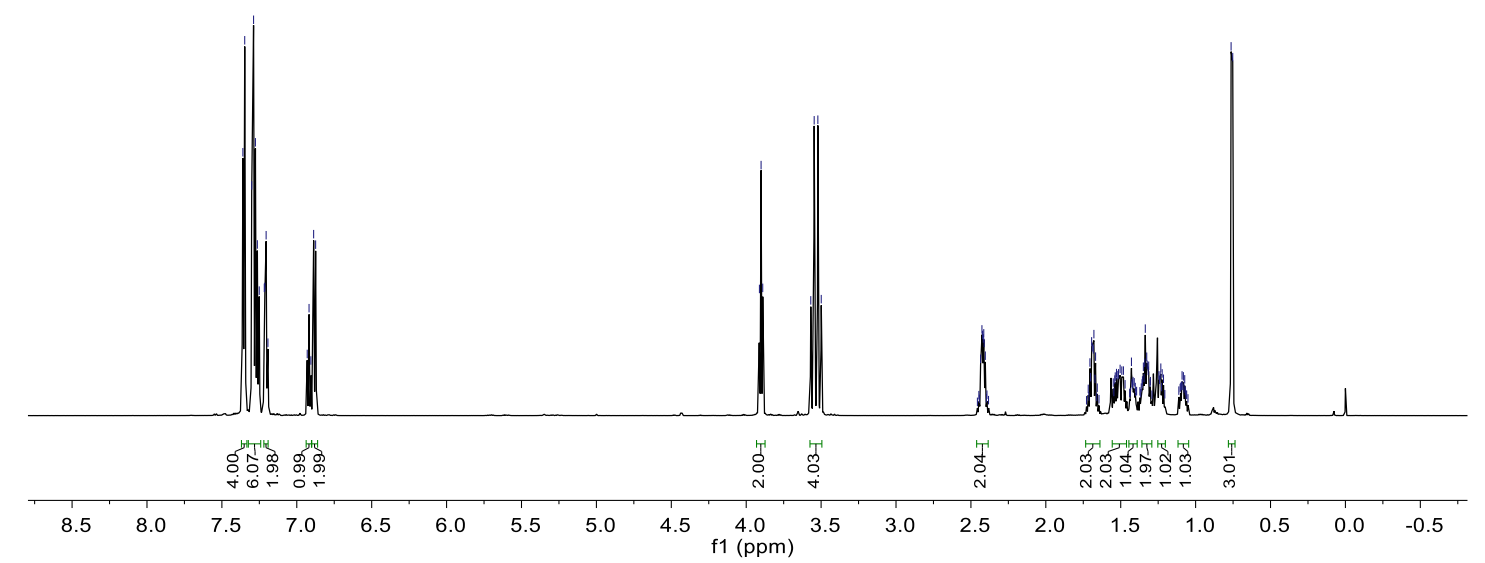

${ }^{13} \mathrm{C}$ NMR (151 MHz, $\left.\mathrm{CDCl}_{3}\right)$<smiles>[B]N(CCC(C)CCCCCOc1ccccc1)Cc1ccccc1</smiles>

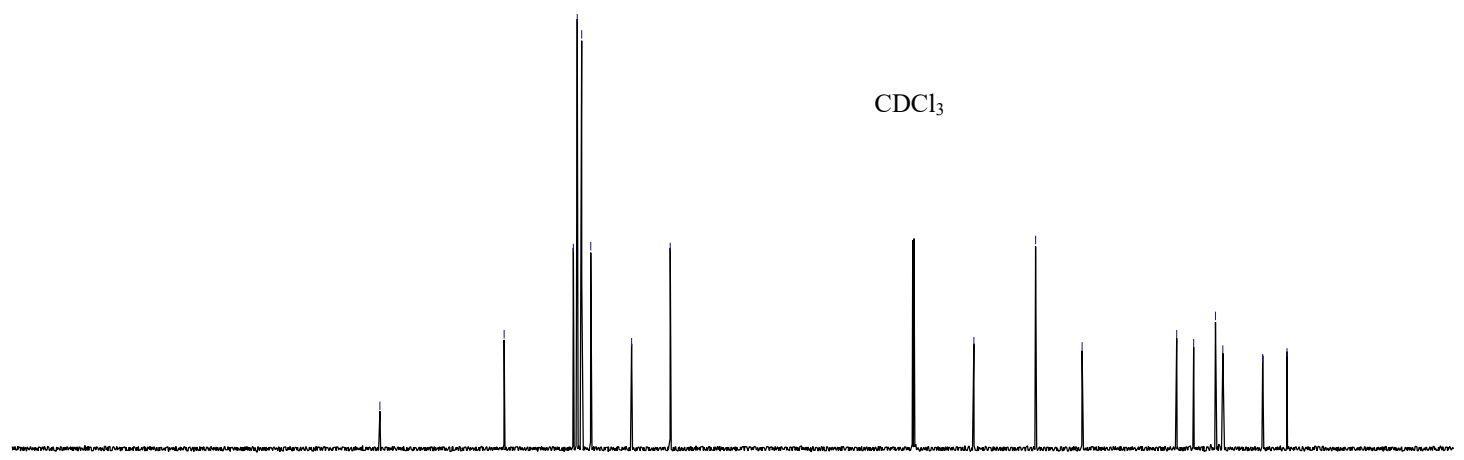

$\begin{array}{lllllllllllllllllllll}210 & 200 & 190 & 180 & 170 & 160 & 150 & 140 & 130 & 120 & \begin{array}{c}110 \\ \mathrm{f} 1(\mathrm{ppm})\end{array} & 100 & 80 & 70 & 60 & 50 & 40 & 30 & 20 & 10 & 0\end{array}$ 


\section{HPLC chromatograms}<smiles>[Y6]C(CC(C)CC=O)(SOP(=O)(O)c1ccccc1)S(=O)(=O)O</smiles>

Data File D: \ChemSta...2-20191114-AS-3-85-15-1ml-80MIN 2019-11-14 20-56-06\023-P2-B6-RAC.D Sample Name: RAC

\begin{tabular}{|c|c|c|c|}
\hline $\begin{array}{l}\text { Acq. Operator } \\
\text { Sample Operator }\end{array}$ & $\begin{array}{l}\text { : SYSTEM } \\
: \text { SYSTEM }\end{array}$ & Seq. Line & 23 \\
\hline Acq. Instrument & $:$ LC & Location & P2-B-06 \\
\hline Injection Date & : 11/15/2019 3:49:19 PM & $\begin{array}{r}\text { Inj } \\
\text { Inj Volume }\end{array}$ & $\begin{array}{c}1 \\
2.000 \mu l\end{array}$ \\
\hline
\end{tabular}

Different Inj Volume from Sample Entry! Actual Inj Volume : $3.000 \mu 1$

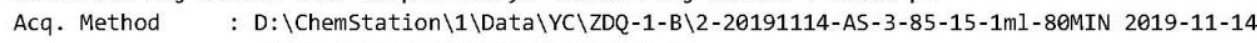
20-56-06\4-AS3-85-15-1ml-80min.M

Last changed : 11/14/2019 9:43:16 AM by SYSTEM

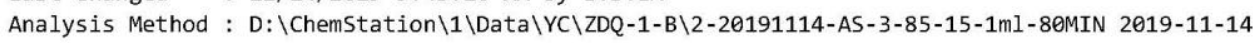
20-56-06\4-AS3-85-15-1ml-80min.M (Sequence Method)

Last changed : 11/16/2019 11:05:27 AM by SYSTEM (modified after loading)

Additional Info: Peak(s) manually integrated

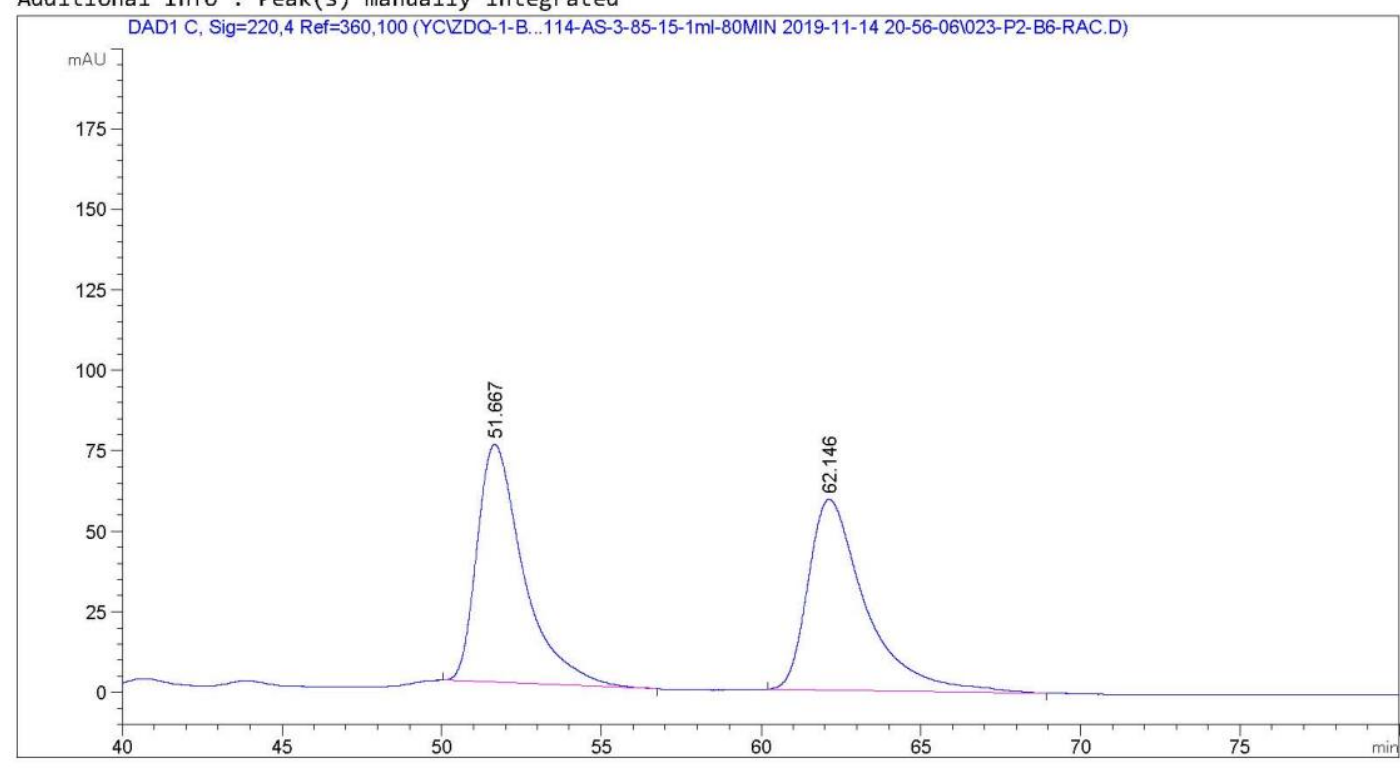

Area Percent Report

$\begin{array}{lll}\text { Sorted By } & : & \text { Signal } \\ \text { Multiplier } & : & 1.0000 \\ \text { Dilution } & : & 1.0000\end{array}$

Use Multiplier \& Dilution Factor with ISTDS

Signal 1: DAD1 C, Sig=220,4 Ref $=360,100$

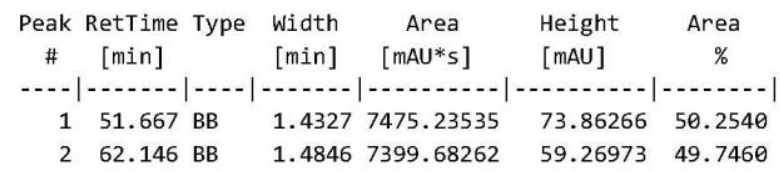


Data File D: \CHEMSTA...20191114-AS-3-85-15-1ML-80MIN 2019-11-14 20-56-06\024-P2-B7-Me-90.D Sample Name: Me-90
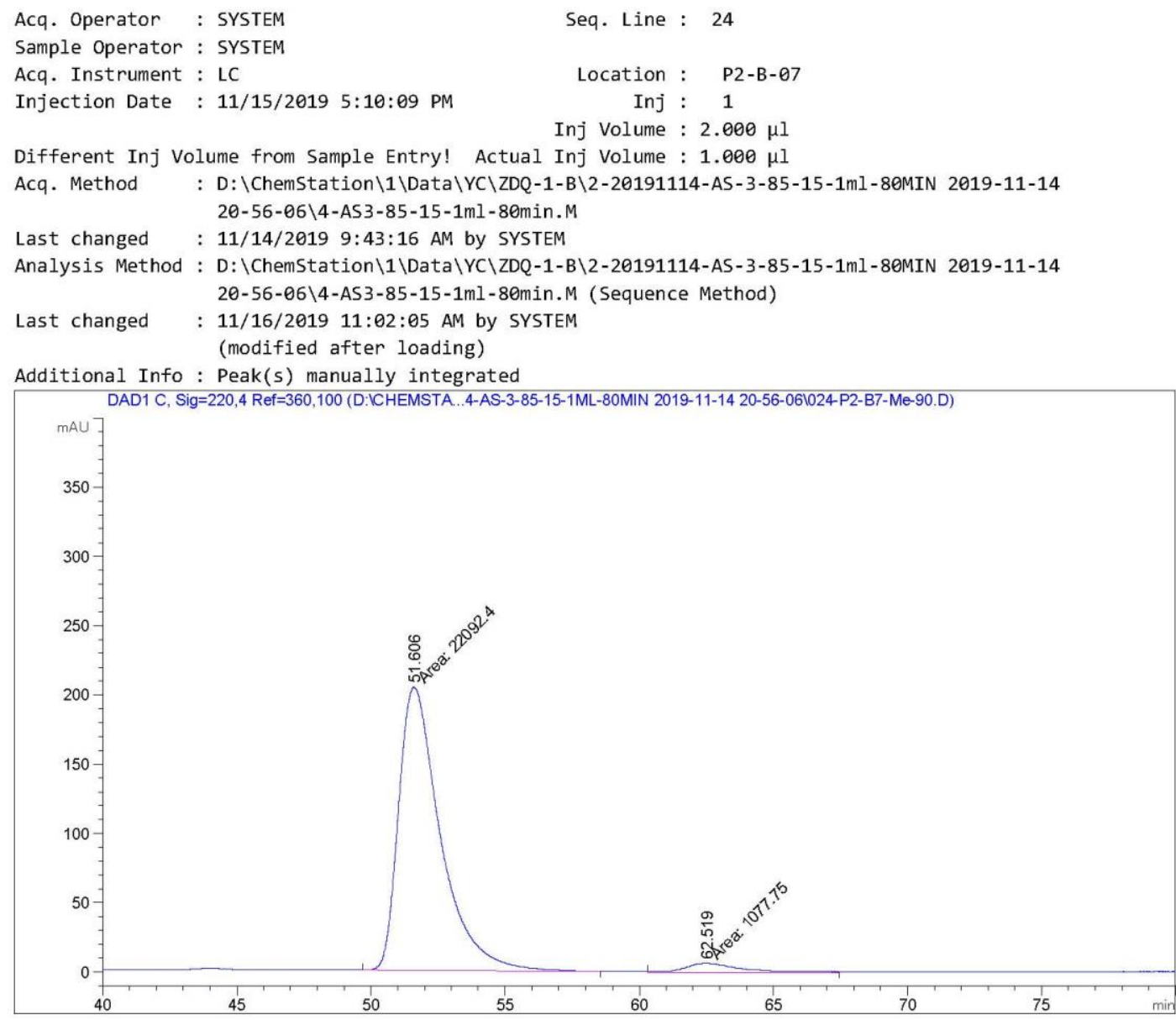

Area Percent Report

\begin{tabular}{|c|c|c|c|c|}
\hline Sorted By & : & Signal & & \\
\hline Multiplier & : & 1.0000 & & \\
\hline Dilution & : & 1.0000 & & \\
\hline \multicolumn{5}{|c|}{ Use Multiplier \& Dilution Factor with ISTDs } \\
\hline \multicolumn{5}{|c|}{ Signal 1: DAD1 C, Sig $=220,4 \operatorname{Re} f=360,100$} \\
\hline $\begin{array}{l}\text { Peak RetTime Type } \\
\# \quad \text { [min] }\end{array}$ & $\begin{array}{l}\text { Width } \\
\text { [min] }\end{array}$ & $\begin{array}{c}\text { Area } \\
{[\mathrm{mAU} * \mathrm{~s}]}\end{array}$ & $\begin{array}{l}\text { Height } \\
{[\mathrm{mAU}]}\end{array}$ & $\begin{array}{c}\text { Area } \\
\%\end{array}$ \\
\hline$---|-----|--\mid$ & $-\cdots--1$ & $|---\cdots|$ & $-\cdots-1$ & $-\ldots$ \\
\hline $151.606 \mathrm{MM}$ & 1.8014 & $2.20924 \mathrm{e} 4$ & 204.40227 & 95.3486 \\
\hline 262.519 MM & 2.6057 & 1077.74524 & 6.89339 & 4.6514 \\
\hline
\end{tabular}


$\mathrm{OHC}$<smiles>CCC(C)CC(CC)(SO)S(=O)(=O)Oc1ccccc1</smiles>

Data File D: \ChemSta...YC\zdq-1-124-2\ZDQ-1-124-2.1 2019-06-20 22-04-07\relacatione0003.D Sample Name: ZDQ-1-124-2
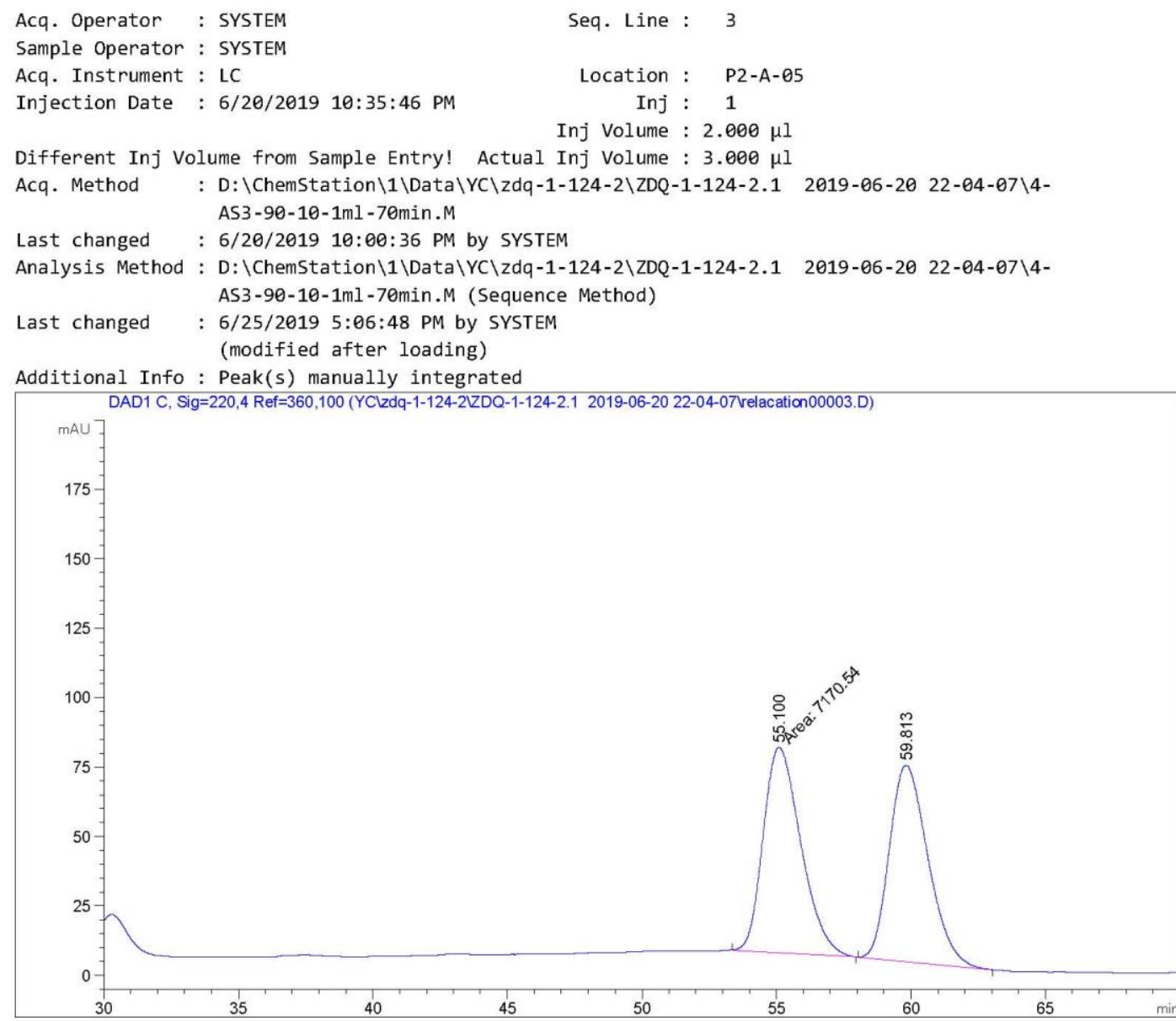

Area Percent Report

\begin{tabular}{|c|c|c|c|c|}
\hline Sorted By & : & Signal & & \\
\hline Multiplier & : & 1.0000 & & \\
\hline Dilution & : & 1.0000 & & \\
\hline \multicolumn{5}{|c|}{ Do not use Multiplier \& Dilution Factor with ISTDs } \\
\hline \multicolumn{5}{|c|}{ Signal 1: DAD1 C, Sig $=220,4$ Ref $=360,100$} \\
\hline $\begin{array}{l}\text { Peak RetTime Type } \\
\# \quad[\mathrm{~min}]\end{array}$ & $\begin{array}{l}\text { Width } \\
{[\text { min] }}\end{array}$ & $\begin{array}{c}\text { Area } \\
{[\mathrm{mAU} * \mathrm{~s}]}\end{array}$ & $\begin{array}{l}\text { Height } \\
{[\mathrm{mAU}]}\end{array}$ & $\begin{array}{c}\text { Area } \\
\%\end{array}$ \\
\hline 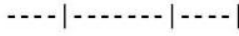 & $-\cdots-\cdots$ & $|-\cdots-\cdots|$ & $-\ldots-\ldots$ & 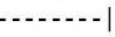 \\
\hline $155.100 \mathrm{MM}$ & 1.6177 & 7170.54053 & 73.87362 & 50.4227 \\
\hline $259.813 \mathrm{BB}$ & 1.5206 & 7050.33057 & 70.63072 & 49.5773 \\
\hline
\end{tabular}




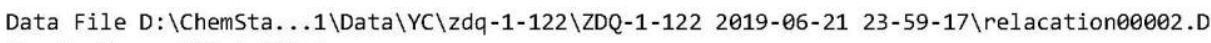
Sample Name: ZDQ-1-122-2
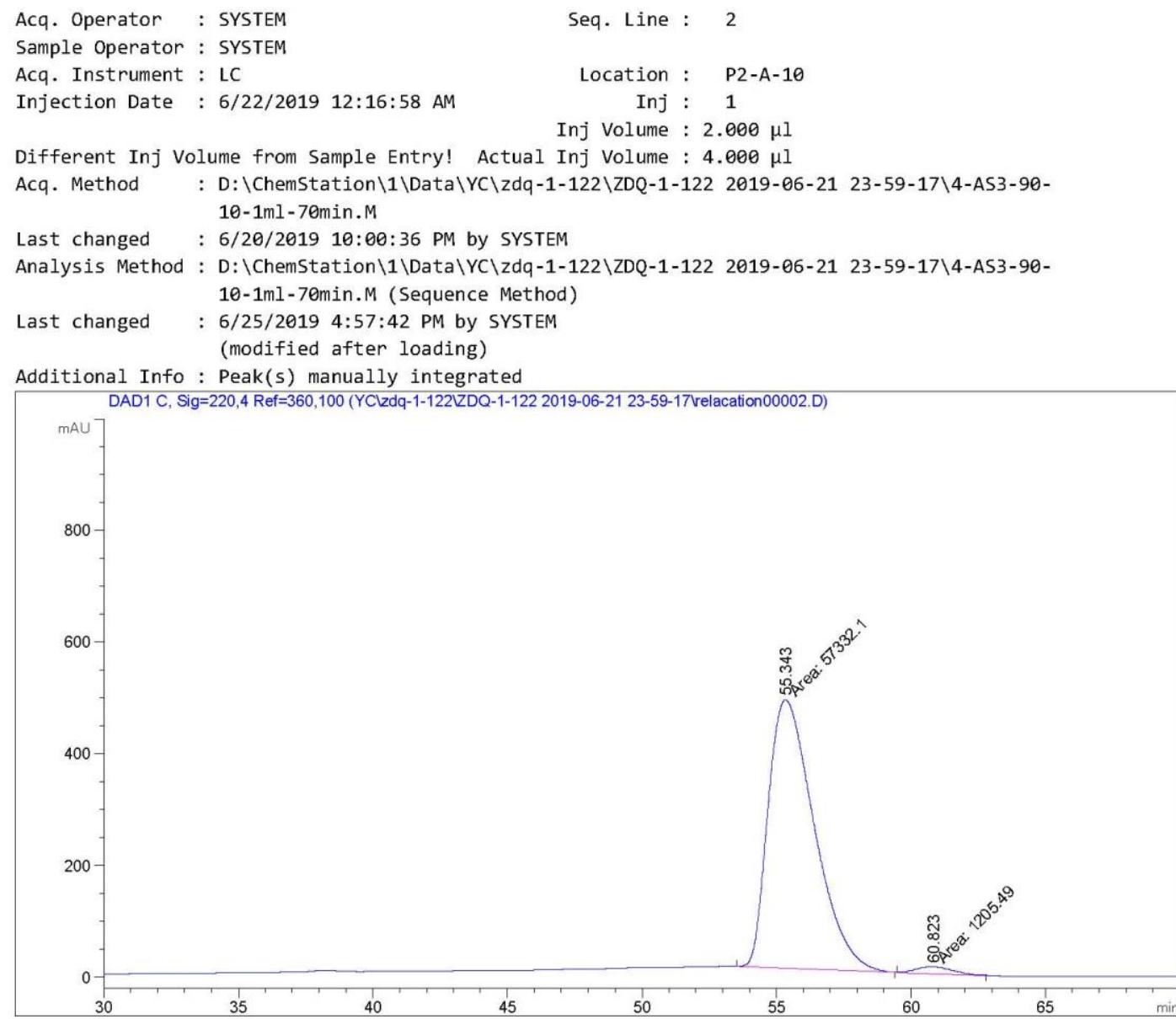

Area Percent Report

\begin{tabular}{|c|c|c|c|c|}
\hline Sorted By & : & Signal & & \\
\hline Multiplier & : & 1.0000 & & \\
\hline Dilution & : & 1.0000 & & \\
\hline \multicolumn{5}{|c|}{ Do not use Multiplier \& Dilution Factor with ISTDs } \\
\hline \multicolumn{5}{|c|}{ Signal 1: DAD1 C, Sig=220,4 $\operatorname{Re} f=360,100$} \\
\hline $\begin{array}{l}\text { Peak RetTime Type } \\
\# \quad \text { [min] }\end{array}$ & $\begin{array}{l}\text { Width } \\
\text { [min] }\end{array}$ & $\begin{array}{c}\text { Area } \\
{[\mathrm{mAU} * \mathrm{~s}]}\end{array}$ & $\begin{array}{l}\text { Height } \\
{[\mathrm{mAU}]}\end{array}$ & $\begin{array}{c}\text { Area } \\
\%\end{array}$ \\
\hline 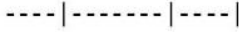 & & 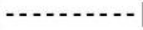 & | & - \\
\hline $155.343 \mathrm{MM}$ & 1.9866 & $5.73321 \mathrm{e} 4$ & 480.99637 & 97.9407 \\
\hline $260.823 \mathrm{MM}$ & 1.5683 & 1205.49365 & 12.81126 & 2.0593 \\
\hline
\end{tabular}


$\underbrace{\mathrm{OHC}}_{\mathrm{SO}_{2} \mathrm{Ph}}$

Data File D: \ChemSta...YC\zdq-1-124-2\ZDQ-1-124-2.1 2019-06-20 22-04-07\relacatione0004.D Sample Name: ZDQ-1-124-3
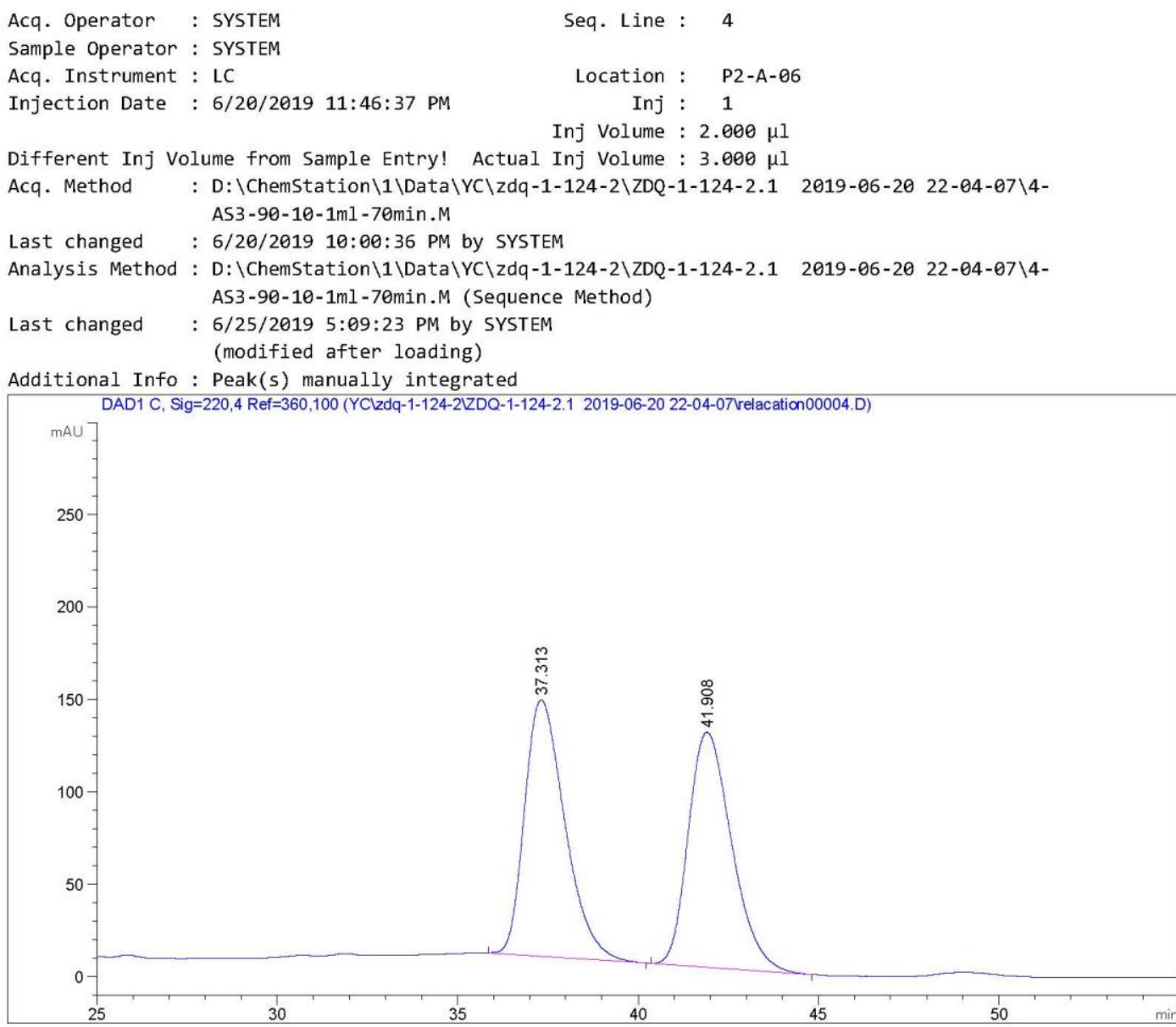

Area Percent Report

$\begin{array}{lll}\text { Sorted By } & : & \text { Signal } \\ \text { Multiplier } & : & 1.0000 \\ \text { Dilution } & : & 1.0000\end{array}$

Do not use Multiplier \& Dilution Factor with ISTDs

Signal 1: DAD1 C, Sig $=220,4$ Ref $=360,100$

\begin{tabular}{cccccc}
$\begin{array}{c}\text { Peak RetTime Type } \\
\text { W } \\
{[\text { min] }}\end{array}$ & $\begin{array}{c}\text { Width } \\
{[\mathrm{min}]}\end{array}$ & $\begin{array}{c}\text { Area } \\
{[\mathrm{mAU} \text { s] }}\end{array}$ & $\begin{array}{c}\text { Height } \\
{[\mathrm{mAU}]}\end{array}$ & $\begin{array}{c}\text { Area } \\
\%\end{array}$ \\
\hline 1 & 37.313 BB & 1.1797 & $1.07477 \mathrm{e} 4$ & 138.75279 & 50.3108 \\
2 & 41.908 BB & 1.2880 & $1.06149 \mathrm{e} 4$ & 127.14460 & 49.6892
\end{tabular}




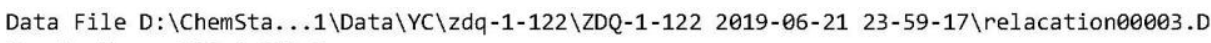
Sample Name: ZDQ-1-122-3
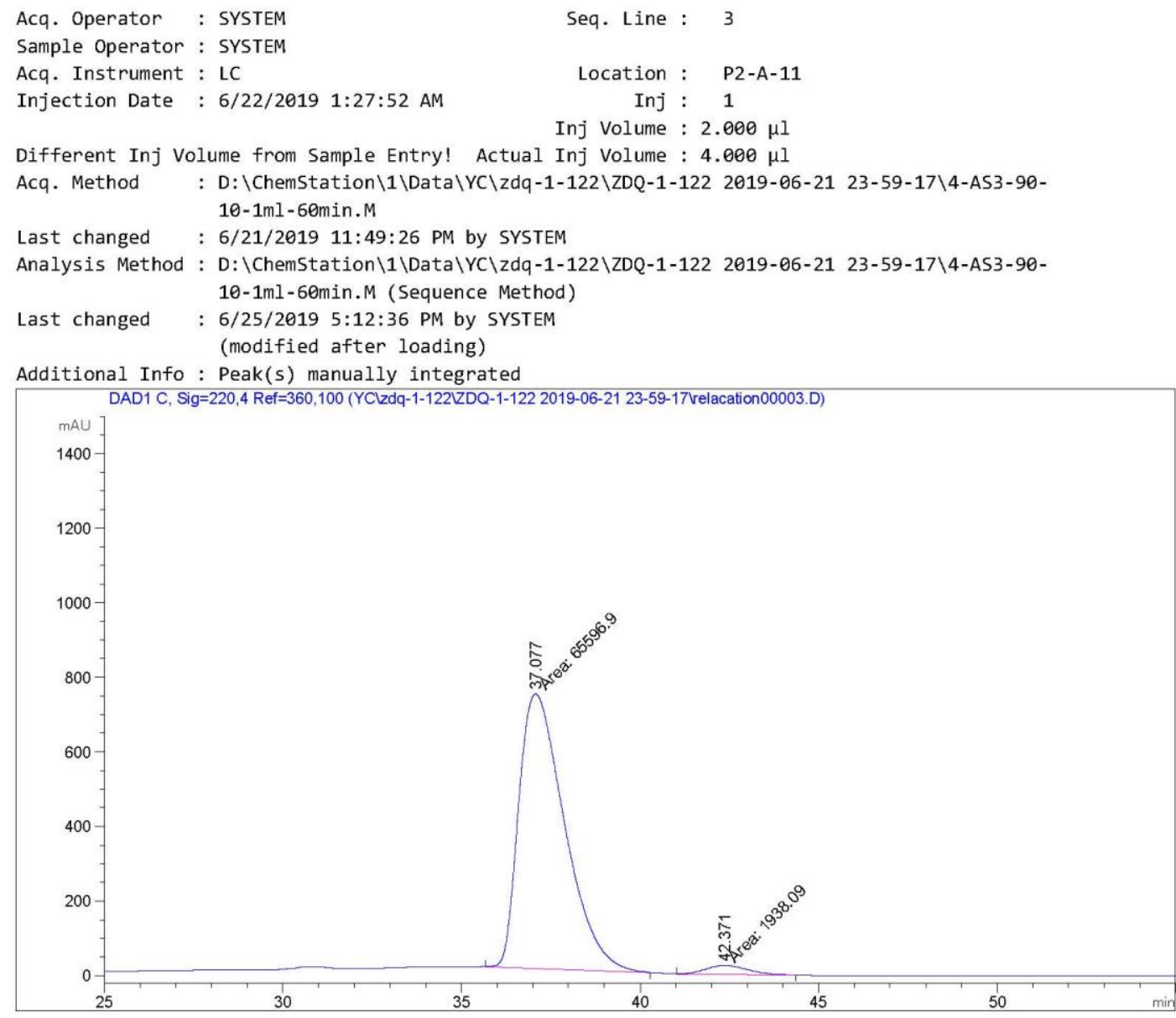

Area Percent Report

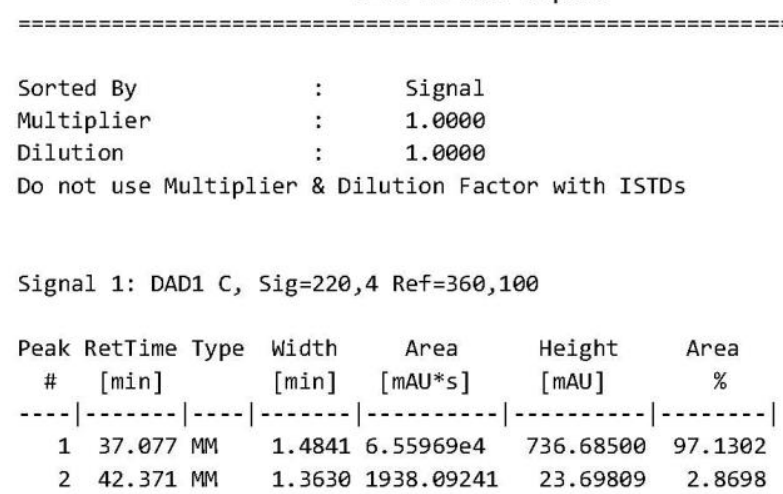




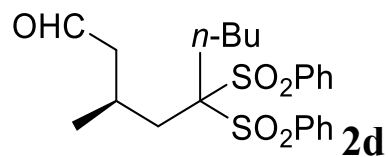

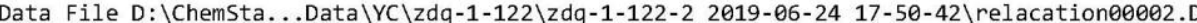
Sample Name: zdq-1-124-4

\begin{tabular}{|c|c|c|c|}
\hline $\begin{array}{l}\text { Acq. Operator } \\
\text { Sample Operator }\end{array}$ & $\begin{array}{l}: \text { SYSTEM } \\
: \text { SYSTEM }\end{array}$ & Seq. Line & 2 \\
\hline Acq. Instrument & : LC & Location & P2-A-05 \\
\hline Injection Date & : 6/24/2019 6:08:22 PM & $\begin{array}{r}\text { Inj } \\
\text { Inj Volume }\end{array}$ & $\begin{array}{lc}: & 1 \\
: & 2.000 \mu 1\end{array}$ \\
\hline
\end{tabular}

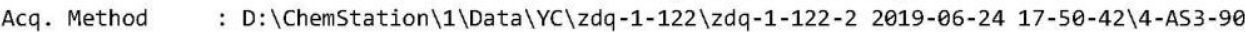
$-10-0.7 \mathrm{ml}-60 \mathrm{~min} . \mathrm{M}$

Last changed : 6/24/2019 5:48:57 PM by SYSTEM

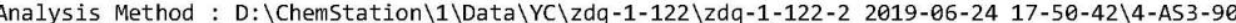

$-10-0.7 \mathrm{ml}-60 \mathrm{~min} . M$ (Sequence Method)

Last changed : 6/25/2019 4:54:30 PM by SYSTEM

(modified after loading)

Additional Info : Peak(s) manually integrated

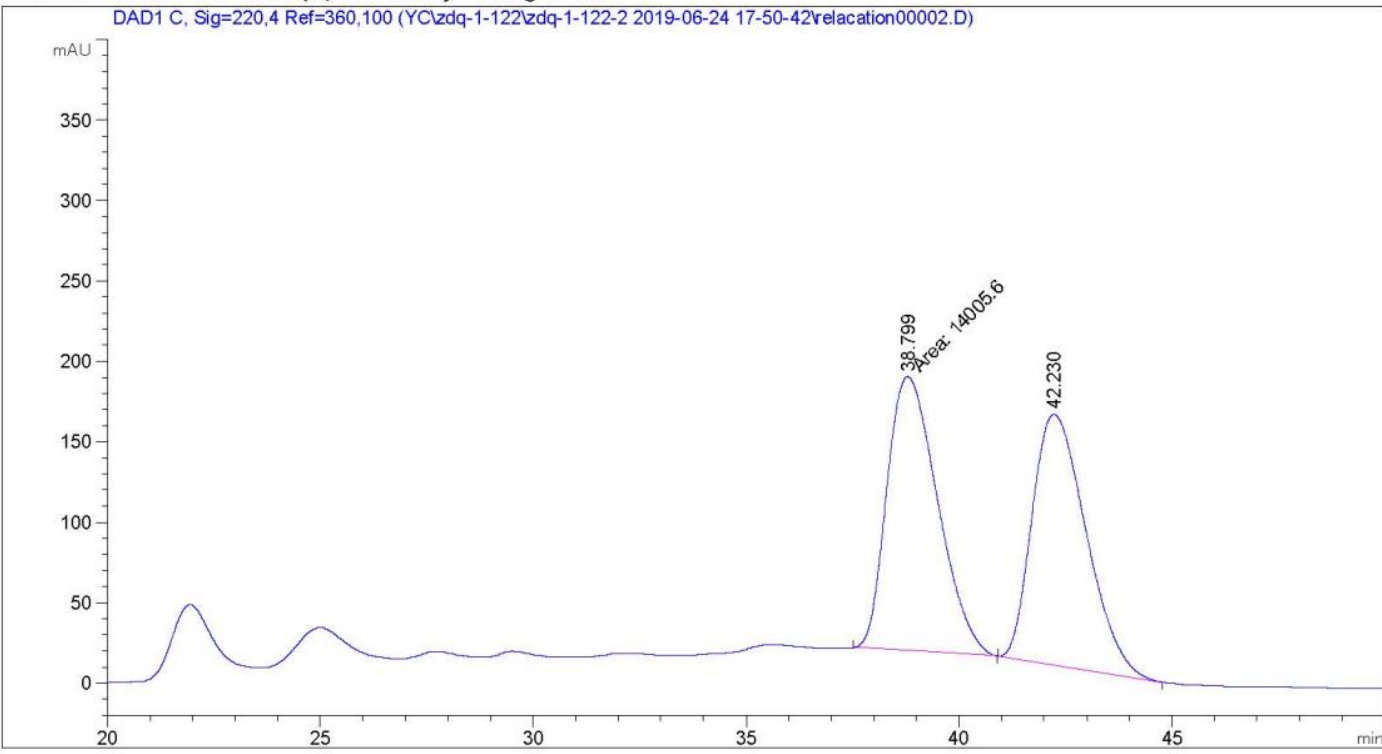

Area Percent Report

\begin{tabular}{|c|c|c|c|c|}
\hline Sorted By & : & \multicolumn{3}{|l|}{ Signal } \\
\hline Multiplier & : & \multicolumn{3}{|l|}{1.0000} \\
\hline Dilution & $:$ & \multicolumn{3}{|l|}{1.0000} \\
\hline \multicolumn{5}{|c|}{ Do not use Multiplier \& Dilution Factor with ISTDs } \\
\hline \multicolumn{5}{|c|}{ Signal 1: DAD1 C, Sig $=220,4$ Ref $=360,100$} \\
\hline $\begin{array}{l}\text { Peak RetTime Type } \\
\# \quad[\text { min] }\end{array}$ & $\begin{array}{l}\text { Width } \\
{[\mathrm{min}]}\end{array}$ & $\begin{array}{c}\text { Area } \\
{[\mathrm{mAU} * \mathrm{~s}]}\end{array}$ & $\begin{array}{l}\text { Height } \\
{[\mathrm{mAU}]}\end{array}$ & $\begin{array}{c}\text { Area } \\
\%\end{array}$ \\
\hline$---|----|-|-|$ & 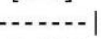 & 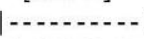 & - & | \\
\hline 138.799 MM & 1.3721 & $1.40056 \mathrm{e} 4$ & 170.12311 & 50.5729 \\
\hline $242.230 \mathrm{BB}$ & 1.3785 & $1.36883 \mathrm{e} 4$ & 155.87410 & 49.4271 \\
\hline Totals : & & $2.76940 \mathrm{e} 4$ & 325.99721 & \\
\hline
\end{tabular}

LC 6/25/2019 4:54:40 PM SYSTEM 
Data File D: \ChemSta...Data\YC\zdq-1-122\zdq-1-122-2 2019-06-24 17-50-42\relacatione0003.D Sample Name: zdq-1-122-4

\begin{tabular}{|c|c|c|c|}
\hline $\begin{array}{l}\text { Acq. Operator } \\
\text { Sample Operator }\end{array}$ & $\begin{array}{l}: \text { SYSTEM } \\
: \text { SYSTEM }\end{array}$ & Seq. Line & 3 \\
\hline Acq. Instrument & : LC & Location & $P 2-A-06$ \\
\hline Injection Date & : 6/24/2019 7:09:12 PM & $\begin{array}{r}\text { Inj } \\
\text { Inj Volume }\end{array}$ & $\begin{array}{cc}: & 1 \\
: & 2.000 \mu l\end{array}$ \\
\hline
\end{tabular}

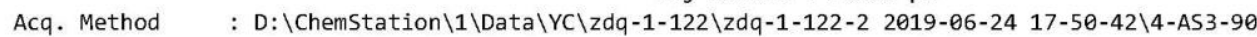
$-10-0.7 \mathrm{ml}-60 \mathrm{~min} . \mathrm{M}$

Last changed : 6/24/2019 5:48:57 PM by SYSTEM

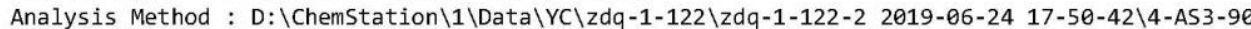

$-10-0.7 \mathrm{ml}-60 \mathrm{~min} . \mathrm{M}$ (Sequence Method)

Last changed : 6/25/2019 4:52:47 PM by SYSTEM

(modified after loading)

Additional Info : Peak(s) manually integrated

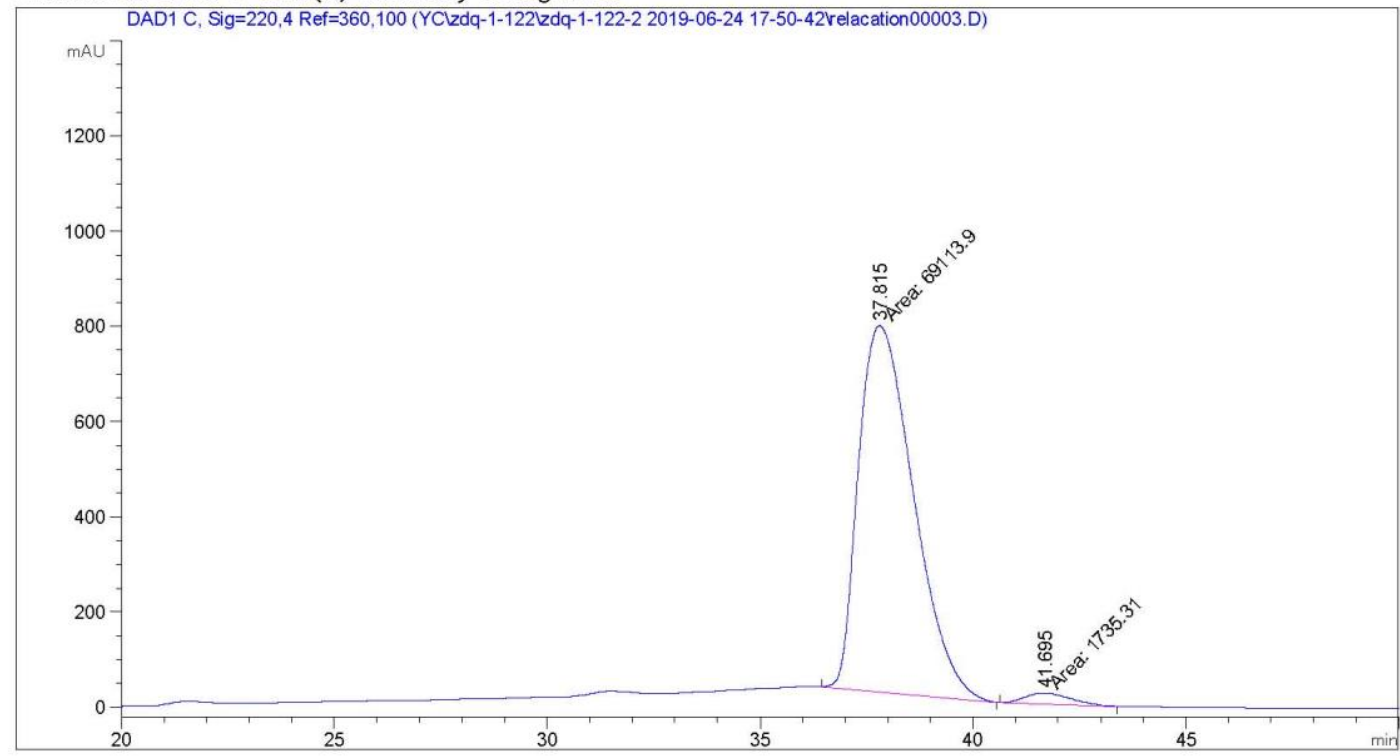

Area Percent Report

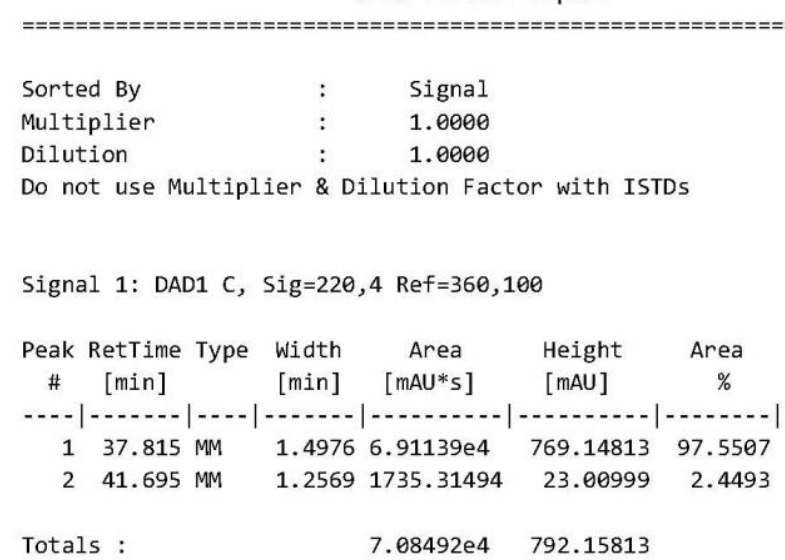

LC 6/25/2019 4:52:56 PM SYSTEM 


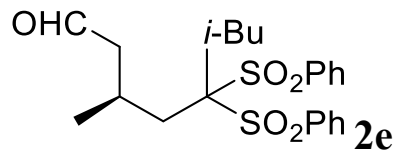

Data File D: \CHEMSTA...-151-RAC\OJ-3-85-15-1ML-70MIN 2019-07-24 10-49-48\relacatione0005.D Sample Name: i-but-rac

\begin{tabular}{|c|c|c|c|}
\hline $\begin{array}{l}\text { Acq. Operator } \\
\text { Sample Operator }\end{array}$ & $\begin{array}{l}\text { : SYSTEM } \\
: \text { SYSTEM }\end{array}$ & Seq. Line & 5 \\
\hline Acq. Instrument & : LC & Location & $P 2-A-08$ \\
\hline Injection Date & : 7/24/2019 2:39:53 PM & $\begin{array}{r}\text { Inj } \\
\text { Inj Volume }\end{array}$ & $\begin{array}{lc}: & 1 \\
: & 2.000 \mu l\end{array}$ \\
\hline
\end{tabular}

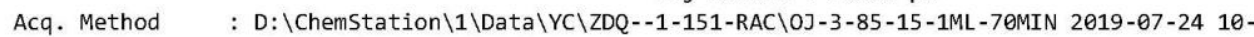
49-48\3-0]3-85-15-1ml-70min.M

Last changed : 4/16/2019 2:44:47 PM by SYSTEM

Analysis Method : D: \ChemStation $\backslash 1 \backslash$ Data \YC\ZDQ--1-151-RAC\0]-3-85-15-1ML-70MIN 2019-07-24 10-

49-48\3-0]3-85-15-1ml-70min.M (Sequence Method)

Last changed : 11/13/2019 $8: 10: 59$ PM by SYSTEM

(modified after loading)

Additional Info : Peak(s) manually integrated

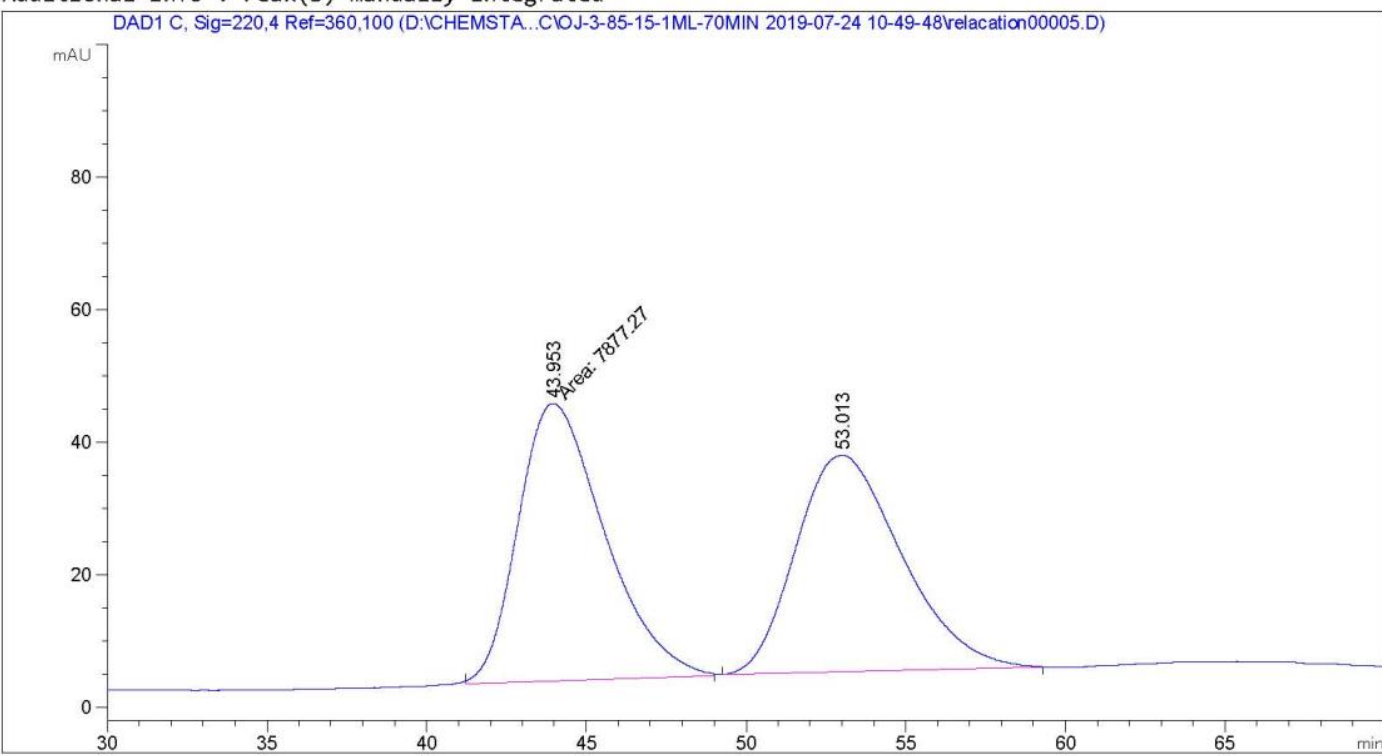

Area Percent Report

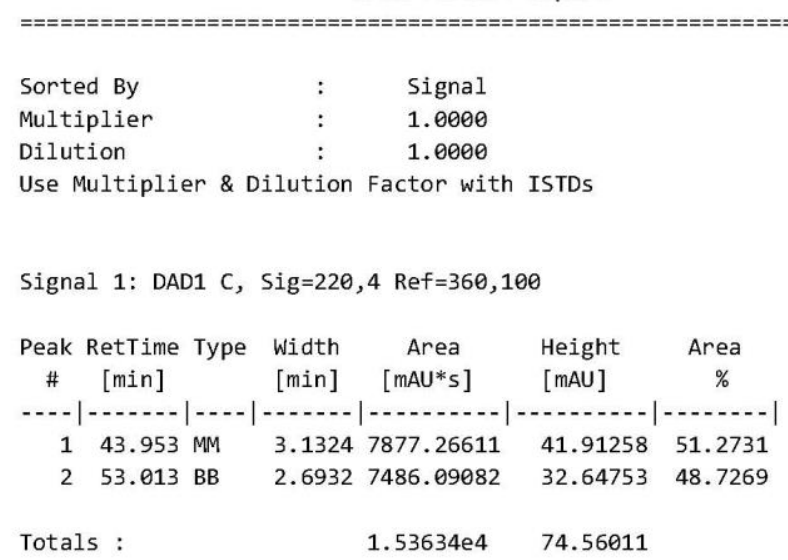




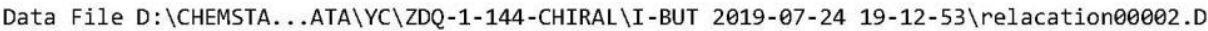
Sample Name: I-BUT

\begin{tabular}{|c|c|c|c|}
\hline $\begin{array}{l}\text { Acq. Operator } \\
\text { Sample Operator }\end{array}$ & $\begin{array}{l}\text { : SYSTEM } \\
: \text { SYSTEM }\end{array}$ & Seq. Line & 2 \\
\hline Acq. Instrument & $:$ LC & Location & $P 2-A-09$ \\
\hline Injection Date & : 7/24/2019 7:29:21 PM & $\begin{array}{r}\text { Inj } \\
\text { Inj Volume }\end{array}$ & $\begin{array}{l}: \quad 1 \\
: 2.000 \mu 1\end{array}$ \\
\hline
\end{tabular}

Different Inj Volume from Sample Entry! Actual Inj Volume : $3.000 \mu 1$

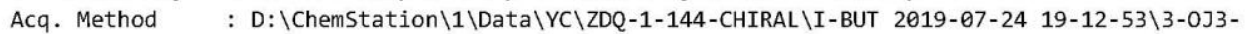
85-15-1ml-70min.M

Last changed : 4/16/2019 2:44:47 PM by SYSTEM

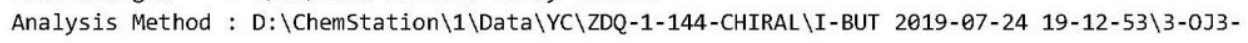
85-15-1ml-70min.M (Sequence Method)

Last changed : 11/13/2019 8:12:31 PM by SYSTEM (modified after loading)

Additional Info : Peak(s) manually integrated

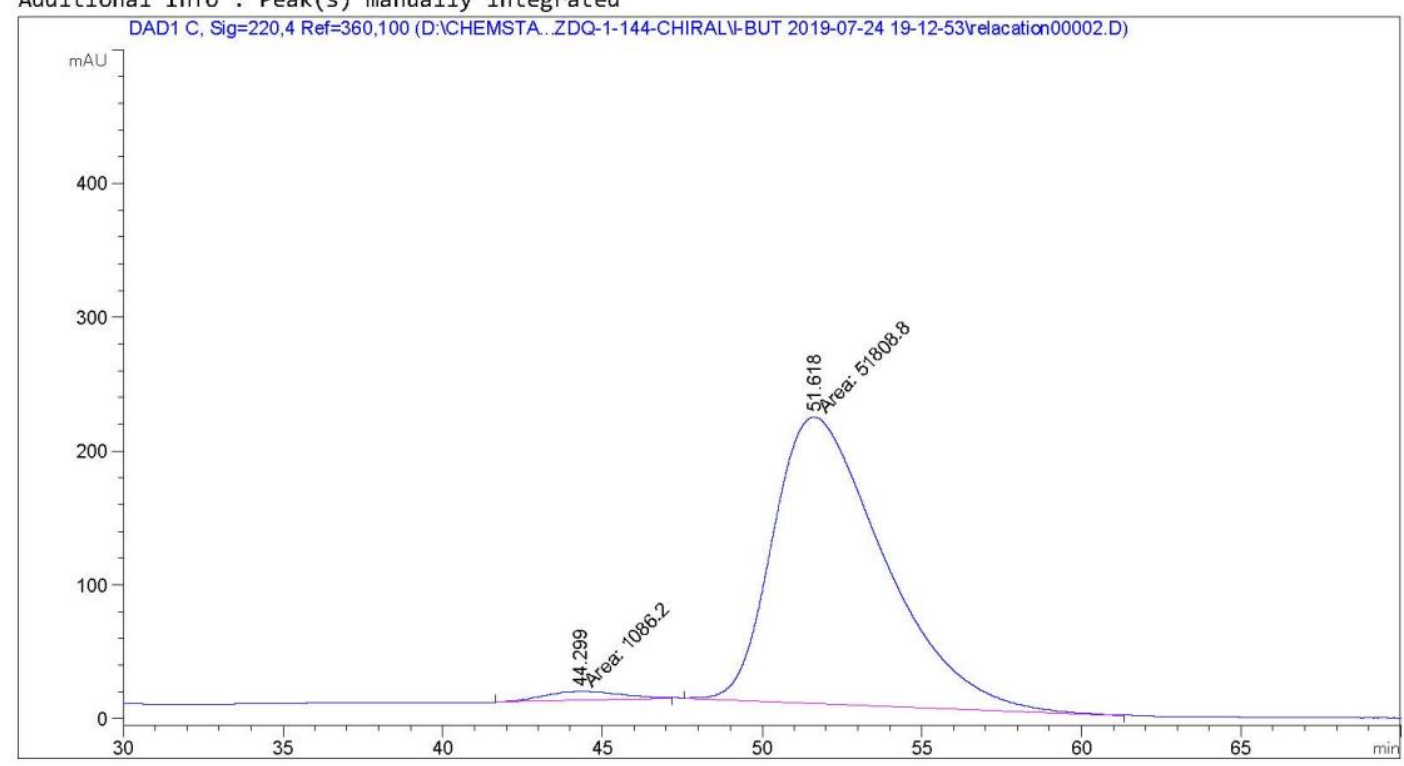

Area Percent Report

\begin{tabular}{|c|c|c|c|c|}
\hline Sorted By & : & Signal & & \\
\hline Multiplier & : & 1.0000 & & \\
\hline Dilution & : & 1.0000 & & \\
\hline \multicolumn{5}{|c|}{ Use Multiplier \& Dilution Factor with ISTDs } \\
\hline \multicolumn{5}{|c|}{ Signal 1: DAD1 C, Sig $=220,4$ Ref $=360,100$} \\
\hline $\begin{array}{l}\text { Peak RetTime Type } \\
\# \quad[\min ]\end{array}$ & $\begin{array}{l}\text { Width } \\
\text { [min] }\end{array}$ & $\begin{array}{c}\text { Area } \\
{[\mathrm{mAU} * \mathrm{~s}]}\end{array}$ & $\begin{array}{l}\text { Height } \\
\text { [mAU] }\end{array}$ & $\begin{array}{c}\text { Area } \\
\%\end{array}$ \\
\hline 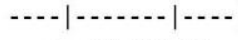 & $-\cdots$ & & & $-\cdots-1$ \\
\hline $144.299 \mathrm{MM}$ & 1.9504 & 1086.20068 & 6.53654 & 2.0535 \\
\hline $251.618 \mathrm{MM}$ & 4.0351 & $5.18088 \mathrm{e} 4$ & 213.99040 & 97.9465 \\
\hline
\end{tabular}




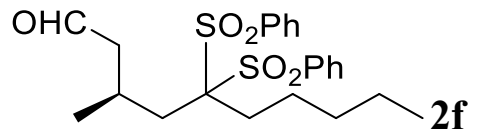

Data File D: \ChemSta...ta\YC\zdq-1-122\zdq-1-122-2 2019-06-24 17-50-42\OnlineEdited--004.D Sample Name: zdq-1-124-5

$\begin{array}{lrl}\text { Acq. Operator } \quad: \text { SYSTEM } & \text { Seq. Line : } & 4 \\ \text { Sample Operator }: \text { SYSTEM } & & \\ \text { Acq. Instrument : LC } & \text { Location : } & \text { P2-A-07 } \\ \text { Injection Date }: 6 / 24 / 20198: 10: 02 \text { PM } & \text { Inj : } & 1 \\ & & \text { Inj Volume : } 2.000 \mu 1\end{array}$

Different Inj Volume from Sample Entry! Actual Inj Volume : $3.000 \mu 1$

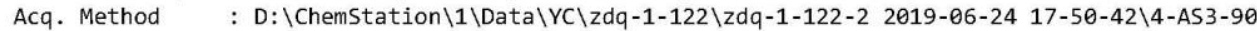
$-10-0.7 \mathrm{ml}-60 \mathrm{~min} . \mathrm{M}$

Last changed : 6/24/2019 8:51:24 PM by SYSTEM (modified after loading)

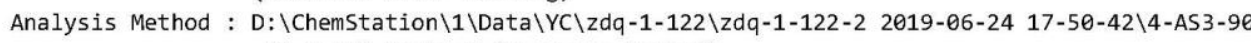
$-10-0.7 \mathrm{ml}-60 \mathrm{~min} . \mathrm{M}$ (Sequence Method)

Last changed : 6/25/2019 4:49:00 PM by SYSTEM (modified after loading)

Additional Info : Peak(s) manually integrated

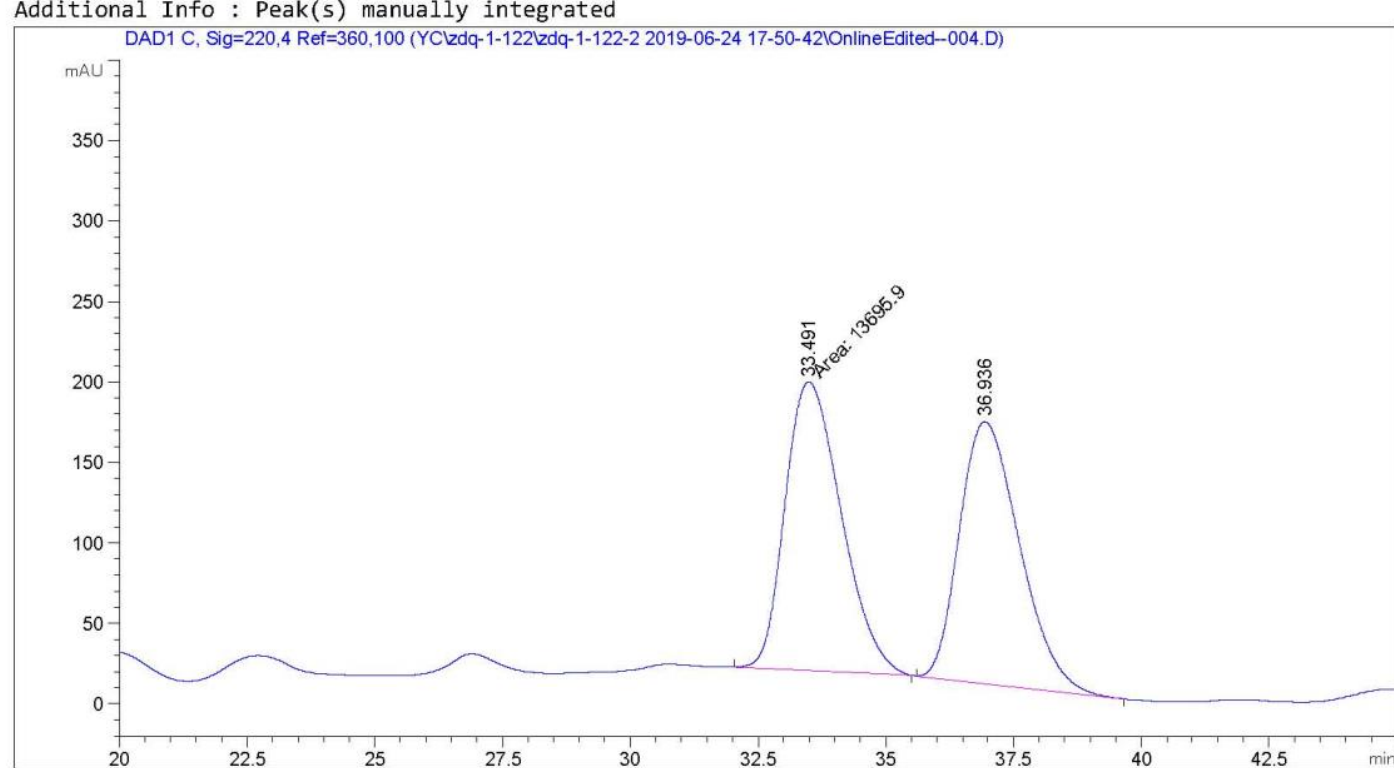

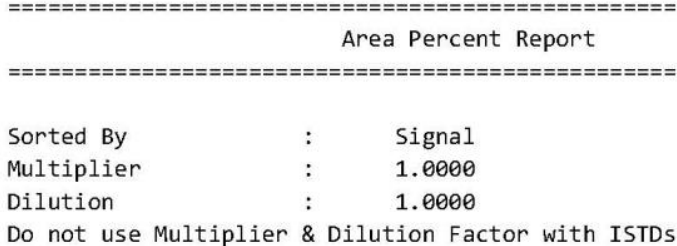

Signal 1: DAD1 C, Sig $=220,4$ Ref $=360,100$

\begin{tabular}{|c|c|c|c|c|c|c|}
\hline $\begin{array}{c}\text { Peak } \\
\#\end{array}$ & $\begin{array}{c}\text { RetTime } \\
\text { [min] }\end{array}$ & Type & $\begin{array}{l}\text { Width } \\
\text { [min] }\end{array}$ & $\begin{array}{c}\text { Area } \\
{[\mathrm{mAU} * \mathrm{~s}]}\end{array}$ & $\begin{array}{l}\text { Height } \\
\text { [mAU] }\end{array}$ & $\begin{array}{c}\text { Area } \\
\%\end{array}$ \\
\hline & & & & $\cdots$ & & $\cdots-$ \\
\hline 1 & 33.491 & MM & 1.2733 & 1. $36959 \mathrm{e} 4$ & 179.26355 & 50.6727 \\
\hline 2 & 36.936 & BB & 1.2722 & $1.33322 \mathrm{e} 4$ & 162.67157 & 49.3273 \\
\hline
\end{tabular}


Data File D: \ChemSta...ta \YC\zdq-1-122\zdq-1-122-2 2019-06-24 17-50-42\OnlineEdited--005.D Sample Name: zdq-1-122-5
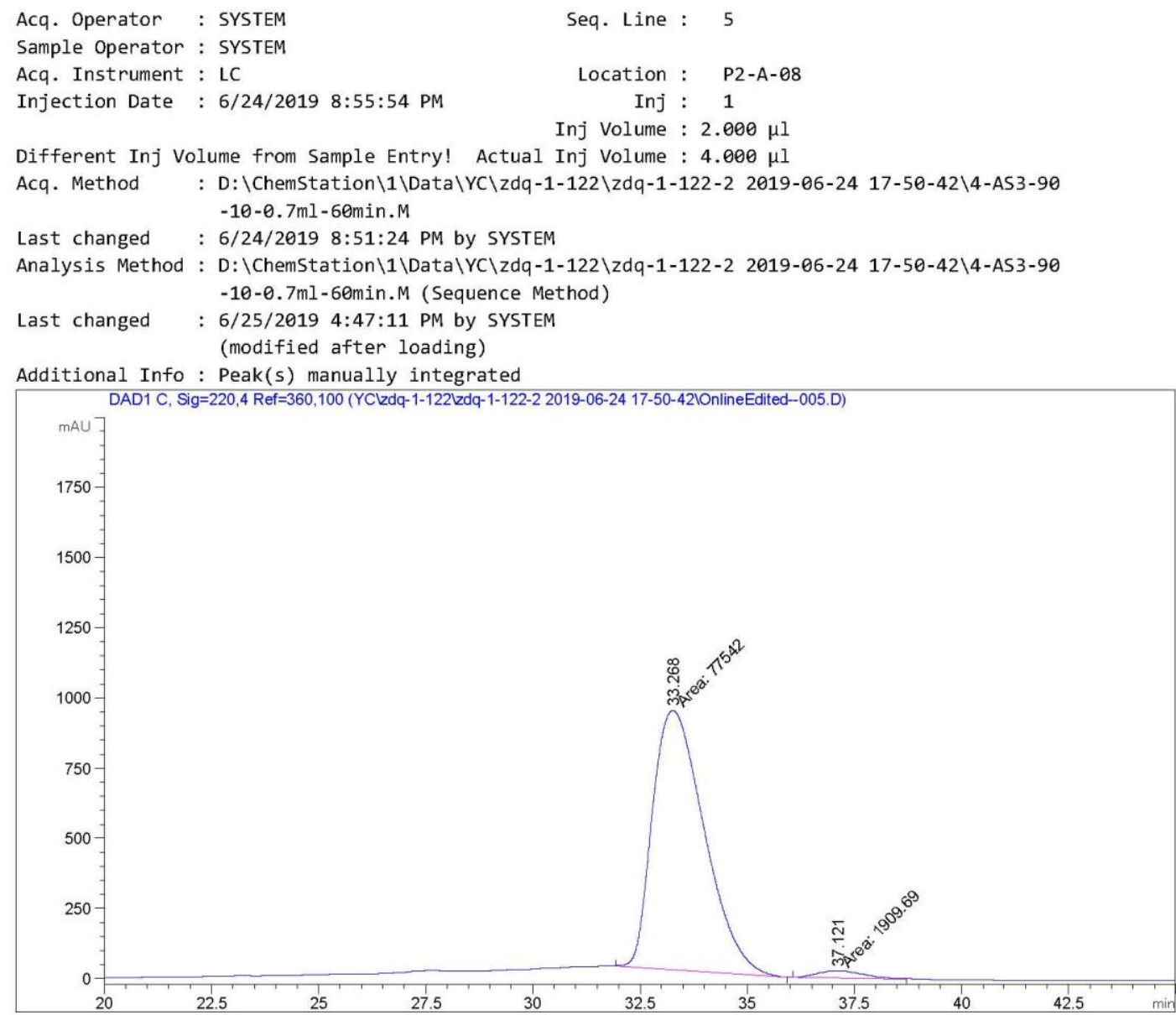

Area Percent Report

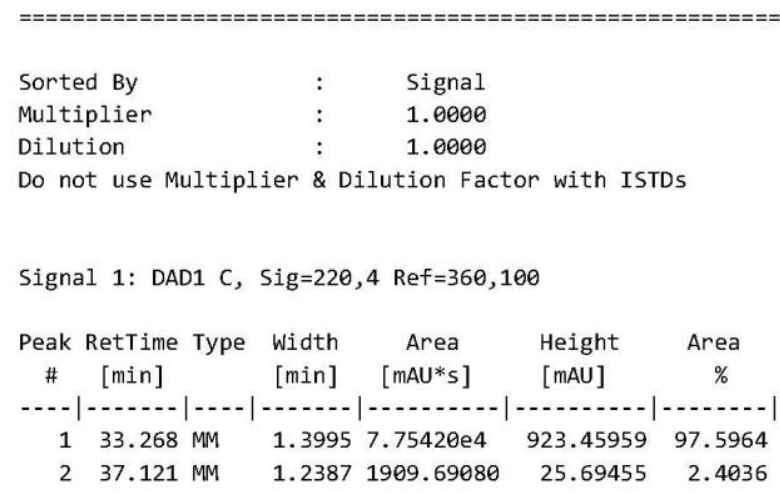


$\mathrm{OHC}$<smiles>CCC(C)CC(F)(F)S(=O)(=O)Oc1ccccc1</smiles>

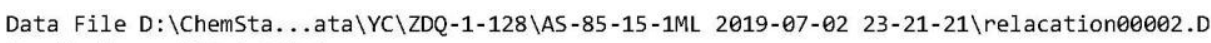
Sample Name: ZDQ-1-129-1

\begin{tabular}{|c|c|c|c|}
\hline $\begin{array}{l}\text { Acq. Operator } \\
\text { Sample Operator }\end{array}$ & $\begin{array}{l}: \text { SYSTEM } \\
: \text { SYSTEM }\end{array}$ & Seq. Line & 2 \\
\hline Acq. Instrument & : LC & Location & $P 2-A-05$ \\
\hline Injection Date & : 7/2/2019 11:39:04 PM & Inj & 1 \\
\hline
\end{tabular}

Acq. Method : D: \ChemStation $\backslash 1 \backslash$ Data $\backslash Y C \backslash Z D Q-1-128 \backslash A S-85-15-1 M L$ 2019-07-02 23-21-21 4 4-AS385-15-1ml-60min.M

Last changed : 4/9/2019 6:45:08 PM by SYSTEM

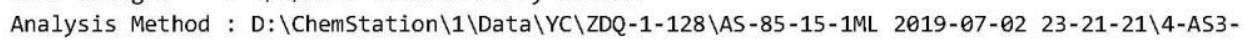
85-15-1ml-60min.M (Sequence Method)

Last changed : 11/13/2019 7:28:20 PM by SYSTEM (modified after loading)

Additional Info : Peak(s) manually integrated

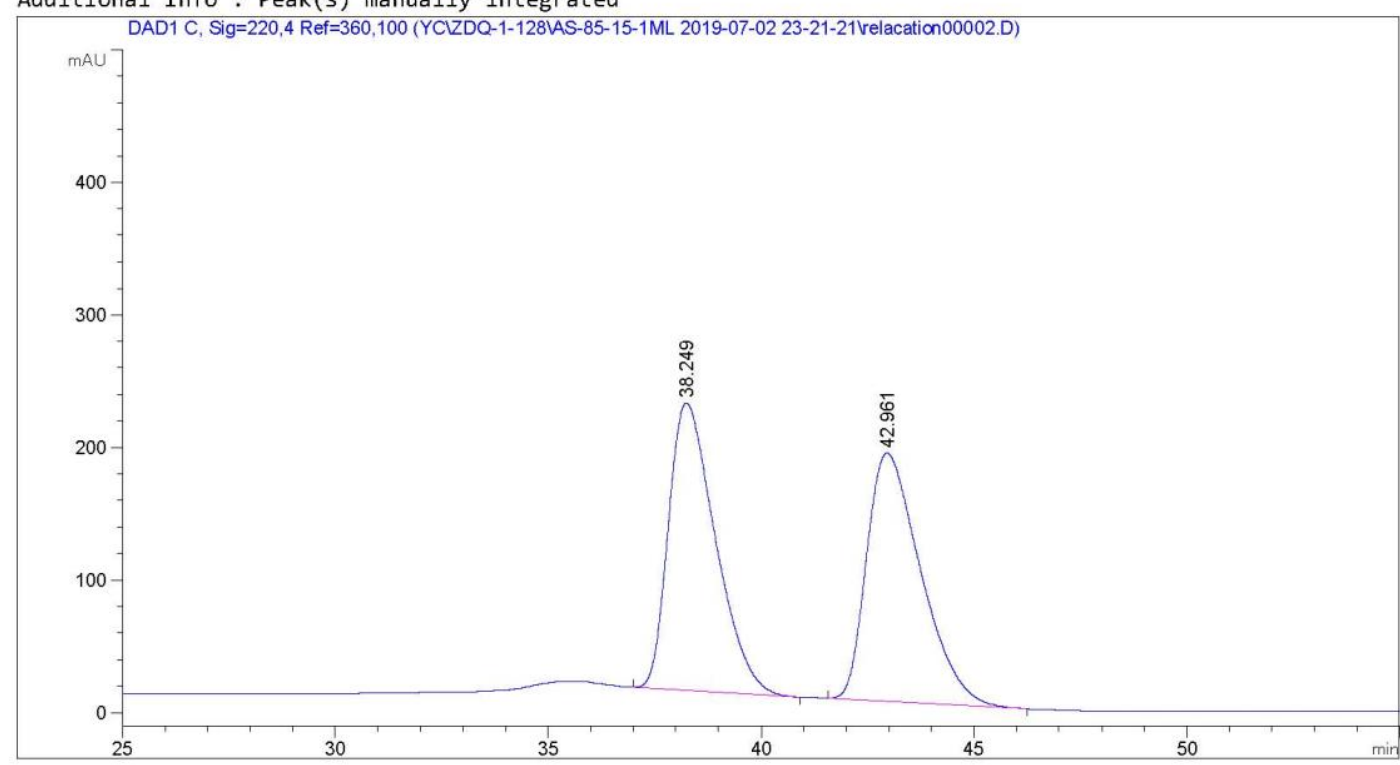

Area Percent Report

\begin{tabular}{|c|c|c|c|c|}
\hline Sorted By & : & Signal & & \\
\hline Multiplier & : & 1.0000 & & \\
\hline Dilution & : & 1.0000 & & \\
\hline \multicolumn{5}{|c|}{ Use Multiplier \& Dilution Factor with ISTDs } \\
\hline \multicolumn{5}{|c|}{ Signal 1: DAD1 C, Sig $=220,4 \operatorname{Re} f=360,100$} \\
\hline $\begin{array}{l}\text { Peak RetTime Type } \\
\#[\text { min] }\end{array}$ & $\begin{array}{l}\text { Width } \\
\text { [min] }\end{array}$ & $\begin{array}{c}\text { Area } \\
{[\mathrm{mAU} * \mathrm{~s}]}\end{array}$ & $\begin{array}{l}\text { Height } \\
{[\mathrm{mAU}]}\end{array}$ & $\begin{array}{c}\text { Area } \\
\%\end{array}$ \\
\hline$---|-n-1-n|$ & $-\ldots-1$ & |-....... & $--2-2-1$ & $-\ldots-n$ \\
\hline 138.249 вB & 1.1555 & $1.65241 \mathrm{e} 4$ & 216.72047 & 50.0147 \\
\hline $242.961 \mathrm{BB}$ & 1.3287 & $1.65144 \mathrm{e} 4$ & 187.29794 & 49.9853 \\
\hline Totals : & & $3.30385 \mathrm{e} 4$ & 404.01842 & \\
\hline
\end{tabular}


Data File D: \ChemSta... ata \YC\ZDQ-1-128\AS-85-15-1ML 2019-07-02 23-21-21\relacatione0003.D Sample Name: ZDQ-1-128-1
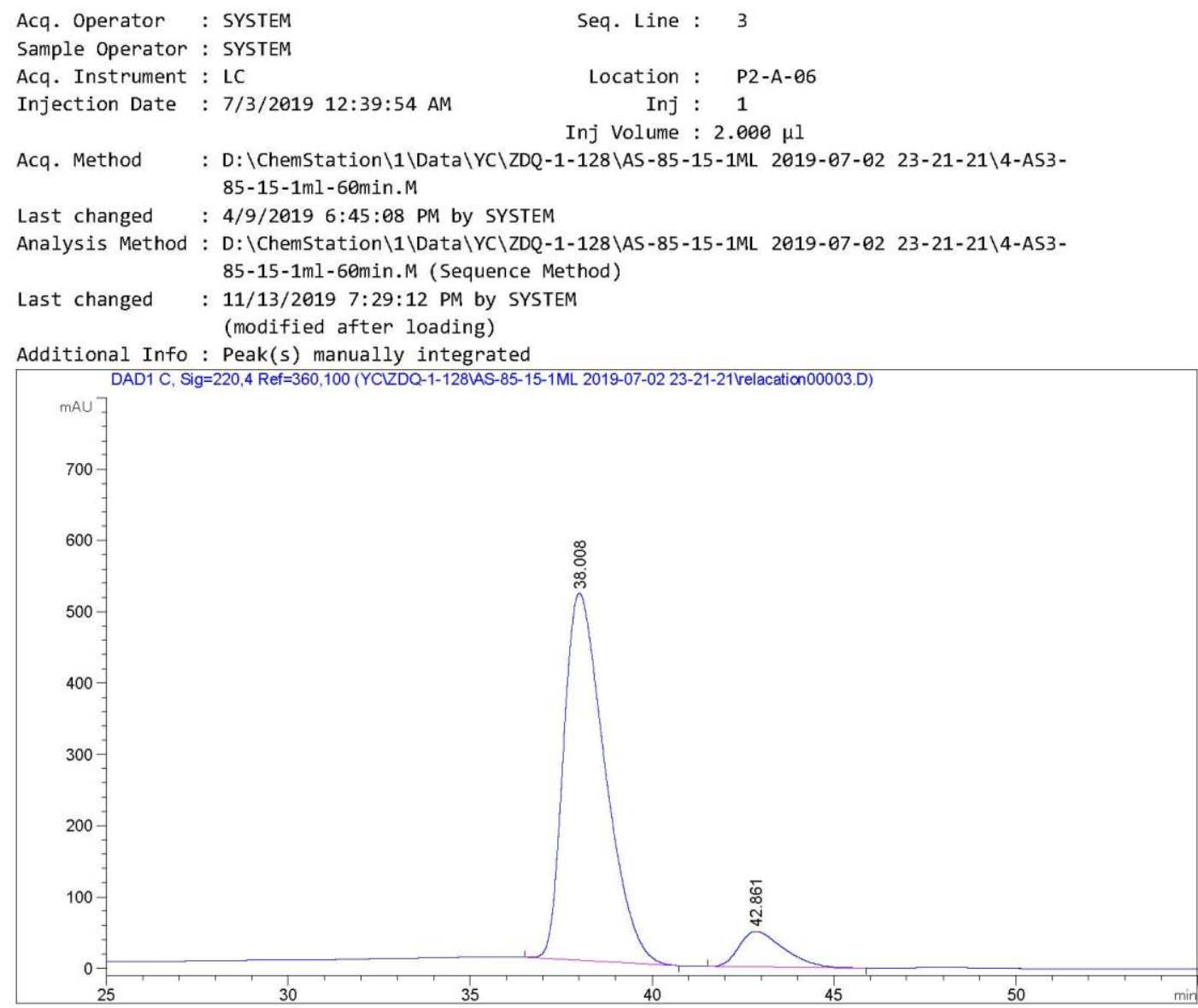

Area Percent Report

\begin{tabular}{|c|c|c|c|c|}
\hline Sorted By & : & Signal & & \\
\hline Multiplier & : & 1.0000 & & \\
\hline Dilution & : & 1.0000 & & \\
\hline \multicolumn{5}{|c|}{ Use Multiplier \& Dilution Factor with ISTDs } \\
\hline \multicolumn{5}{|c|}{ Signal 1: DAD1 C, Sig $=220,4 \operatorname{Re} f=360,100$} \\
\hline $\begin{array}{l}\text { Peak RetTime Type } \\
\# \text { [min] }\end{array}$ & $\begin{array}{l}\text { Width } \\
\text { [min] }\end{array}$ & $\begin{array}{c}\text { Area } \\
{[\mathrm{mAU} * \mathrm{~s}]}\end{array}$ & $\begin{array}{l}\text { Height } \\
\text { [mAU] }\end{array}$ & $\begin{array}{c}\text { Area } \\
\%\end{array}$ \\
\hline$-\cdots|-----|--\mid$ & $-\ldots-n$ & $|-\ldots+n|$ & $-2-2-1$ & $-\ldots-1$ \\
\hline $138.008 \mathrm{BB}$ & 1.1944 & $4.03391 \mathrm{e} 4$ & 514.67230 & 90.4980 \\
\hline $242.861 \mathrm{BB}$ & 1.2449 & 4235.50244 & 50.06968 & 9.5020 \\
\hline Totals : & & $4.45746 \mathrm{e} 4$ & 564.74198 & \\
\hline
\end{tabular}

LC 11/13/2019 7:29:17 PM SYSTEM 


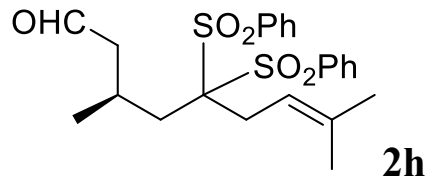

Data File D: \CHEM32\...OD-H-90-10-1ML-45MIN 2019-07-24 18-55-35\002-P2-A6-TRI-OLIFIN-RAC.D Sample Name: TRI-OLIFIN-RAC

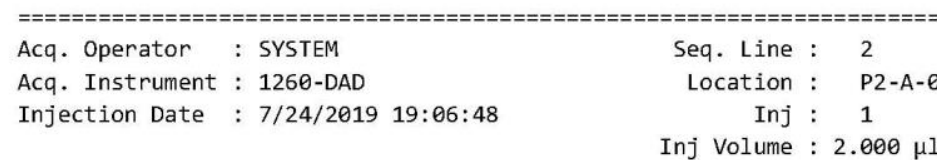

Different Inj Volume from Sample Entry! Actual Inj Volume : $3.000 \mu 1$

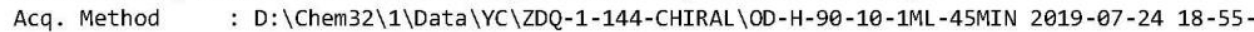
$35 \backslash Z D Q-O D-H-90-10-1 M L-45 M I N . M$

Last changed : 7/24/2019 18:52:26 by SYSTEM

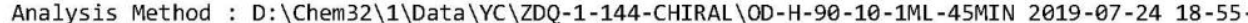
$35 \backslash Z D Q-O D-H-90-10-1 M L-45 M I N . M$ (Sequence Method)

Last changed : 11/12/2019 20:55:53 by SYSTEM

(modified after loading)

Additional Info : Peak(s) manually integrated

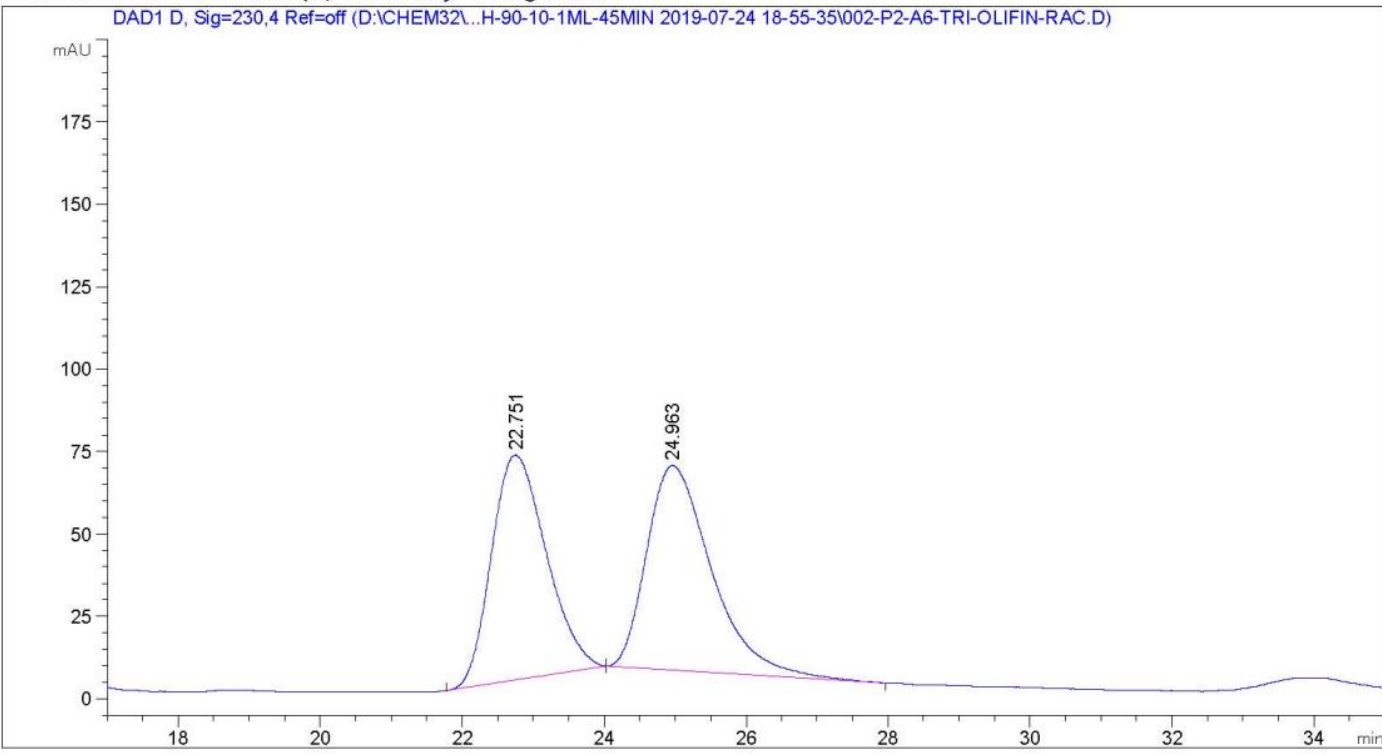

Area Percent Report

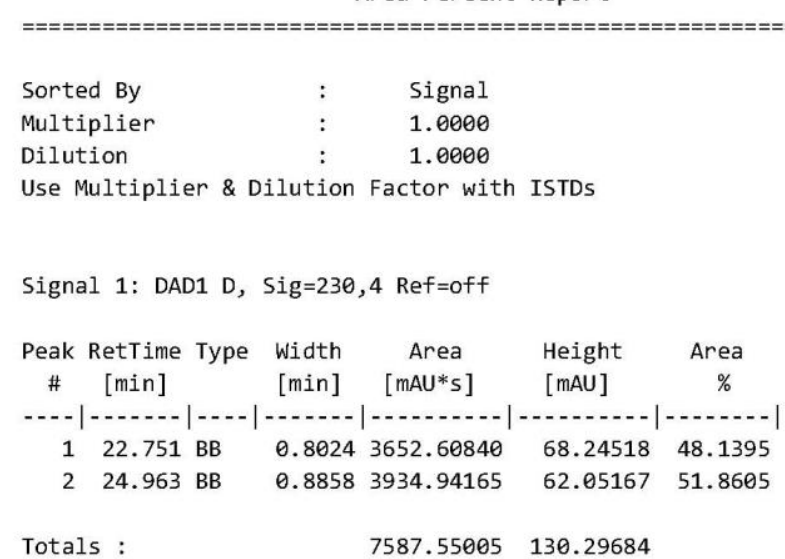

1260-DAD 11/12/2019 20:56:03 SYSTEM 
Data File D: \Chem32\...RAL \OD-H-90-10-1ML-45MIN 2019-07-24 18-55-35\003-P2-A7-TRI-OLIFIN.D Sample Name: TRI-OLIFIN

\begin{tabular}{|c|c|c|c|}
\hline Acq. Operator & : SYSTEM & Seq. Line : & 3 \\
\hline Acq. Instrument & : 1260-DAD & Location : & $P 2-A-\theta$ \\
\hline Injection Date & : 7/24/2019 19:52:39 & $\begin{array}{r}\text { Inj : } \\
\text { Inj Volume : }\end{array}$ & $\begin{array}{l}1 \\
.000\end{array}$ \\
\hline
\end{tabular}

Different Inj Volume from Sample Entry! Actual Inj Volume : $3.000 \mu 1$

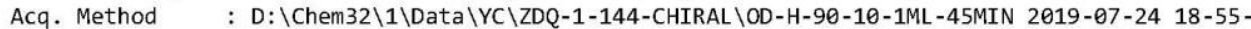
$35 \backslash Z D Q-O D-H-90-10-1 M L-45 M I N . M$

Last changed : 7/24/2019 18:52:26 by SYSTEM

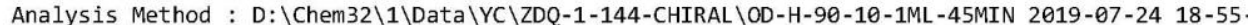
$35 \backslash Z D Q-O D-H-90-10-1 M L-45 M I N . M$ (Sequence Method)

Last changed : 11/12/2019 20:57:37 by SYSTEM

(modified after loading)

Additional Info : Peak(s) manually integrated

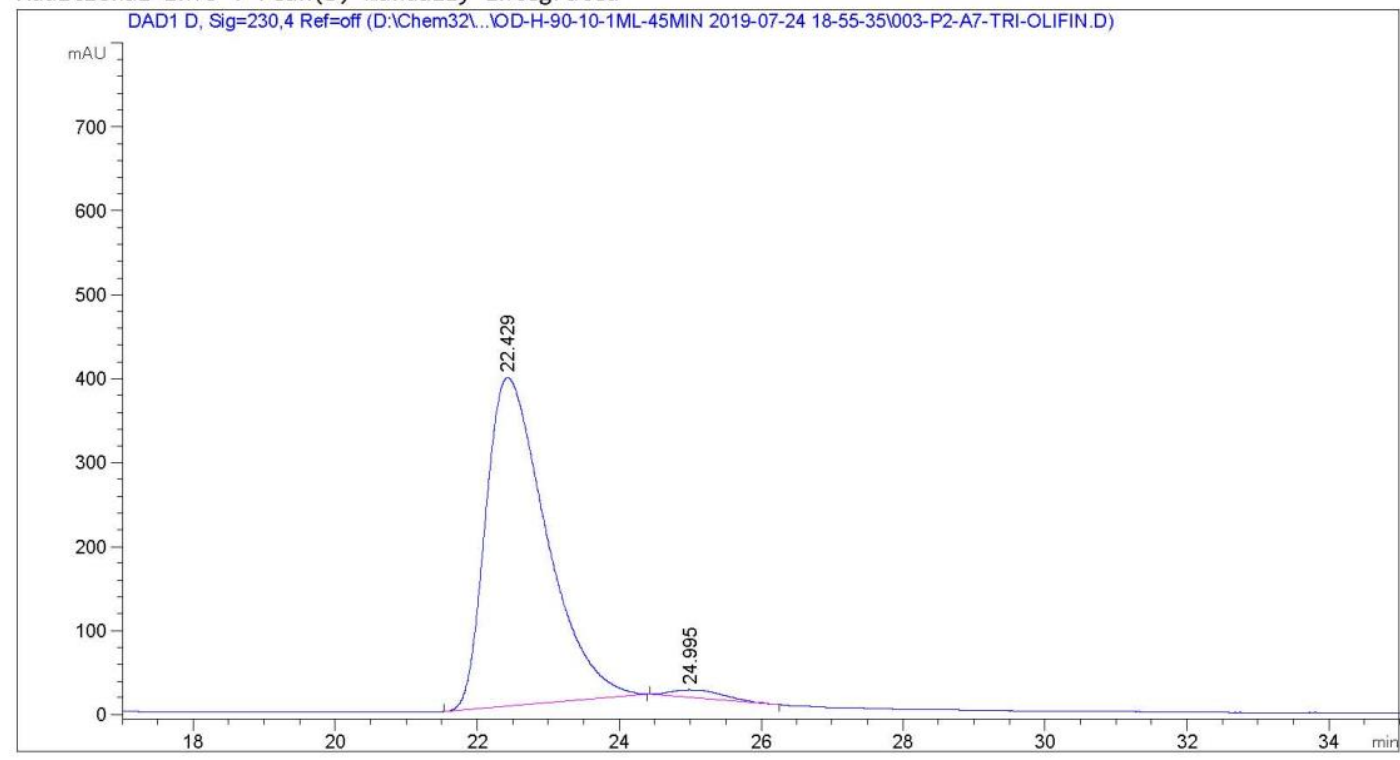

Area Percent Report

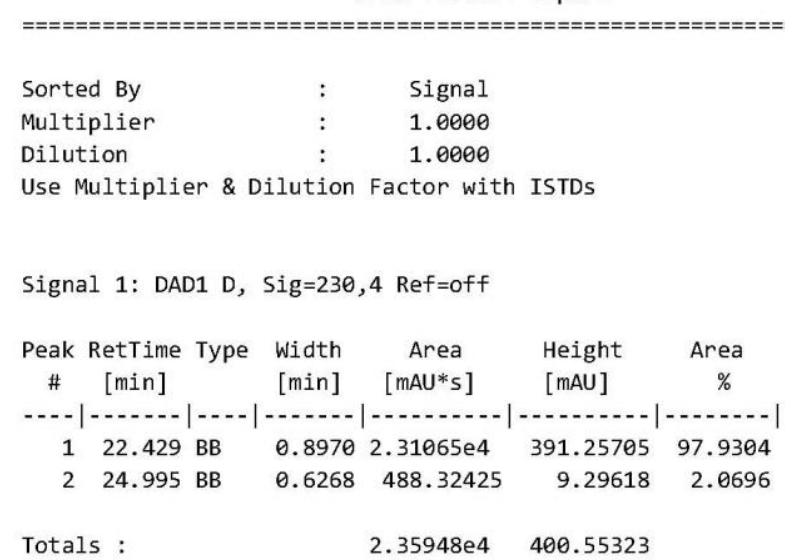

1260-DAD 11/12/2019 20:57:43 SYSTEM 
$\underbrace{\mathrm{OHC}}_{\mathrm{SO}_{2} \mathrm{Ph}} \mathrm{SO}_{2} \mathrm{Ph}$

Data File D: \ChemSta...-CHIRAL \AS-3-89-9-0.9ML-60min 2019-09-03 09-28-32\relacatione0002.D Sample Name: I-PROP-RAC

$\begin{array}{lrl}\text { Acq. Operator } \quad: \text { SYSTEM } & \text { Seq. Line : } & \\ \text { Sample Operator }: \text { SYSTEM } & \text { Location : } & \\ \text { Acq. Instrument }: \text { LC } & \text { Inj : } 01 & 1 \\ \text { Injection Date }: 9 / 3 / 20199: 41: 17 \mathrm{AM} & \text { Inj Volume : } 2.000 \mu \mathrm{l}\end{array}$

Different Inj Volume from Sample Entry! Actual Inj Volume : $15.000 \mu 1$

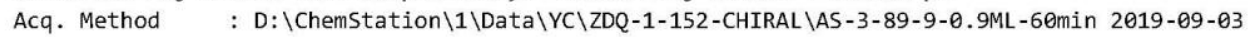
09-28-32\4-AS3-89-9-1ml-60min.M

Last changed : 9/3/2019 9:25:44 AM by SYSTEM

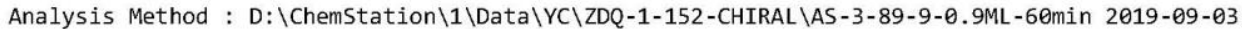
09-28-32 \4-AS3-89-9-1ml-60min.M (Sequence Method)

Last changed : 11/13/2019 8:22:03 PM by SYSTEM

(modified after loading)

Additional Info : Peak(s) manually integrated

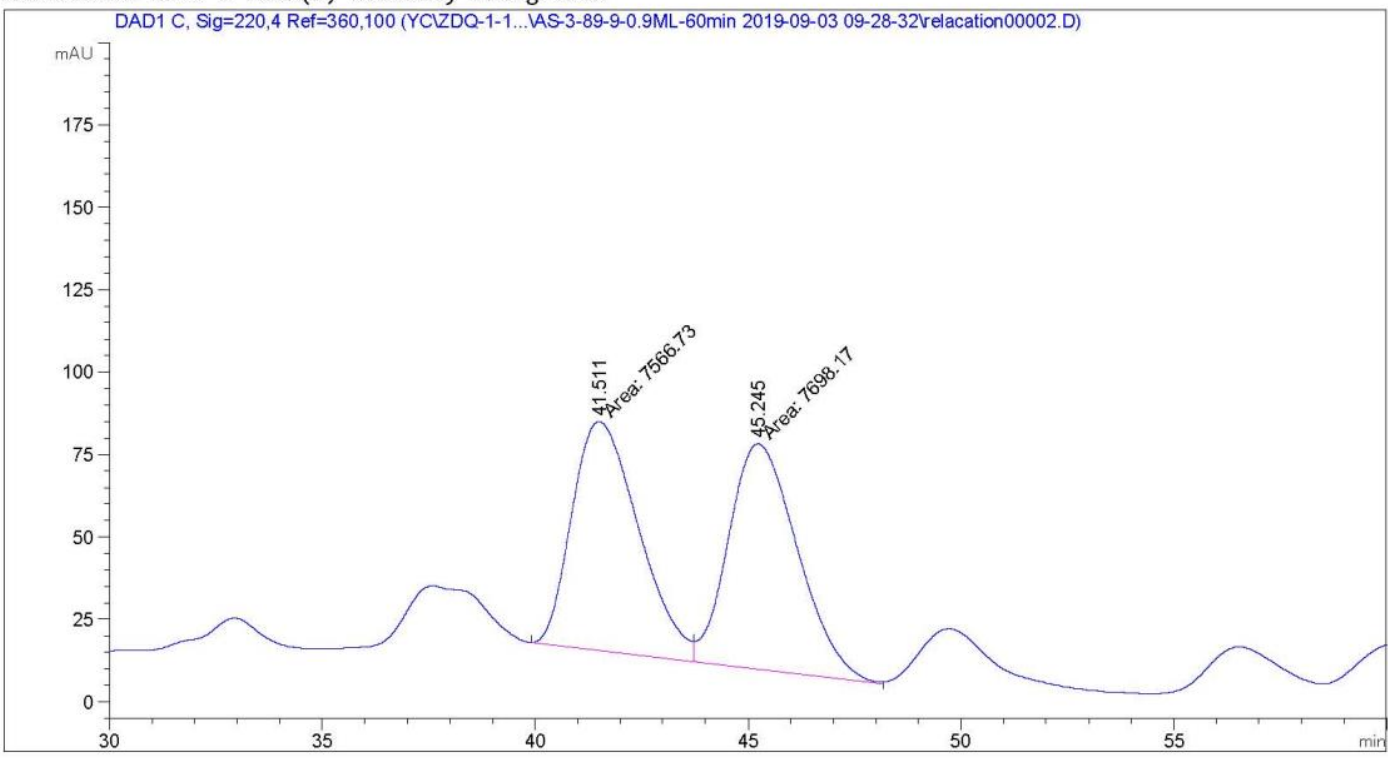

Area Percent Report

\begin{tabular}{|c|c|c|c|c|}
\hline Sorted By & : & Signal & & \\
\hline Multiplier & : & 1.0000 & & \\
\hline Dilution & : & 1.0000 & & \\
\hline \multicolumn{5}{|c|}{ Use Multiplier \& Dilution Factor with ISTDs } \\
\hline \multicolumn{5}{|c|}{ Signal 1: DAD1 C, Sig=220,4 $\operatorname{Ref}=360,100$} \\
\hline $\begin{array}{l}\text { Peak RetTime Type } \\
\# \quad \text { [min] }\end{array}$ & $\begin{array}{l}\text { Width } \\
\text { [min] }\end{array}$ & $\begin{array}{c}\text { Area } \\
{[\mathrm{mAU} * \mathrm{~s}]}\end{array}$ & $\begin{array}{l}\text { Height } \\
\text { [mAU] }\end{array}$ & $\begin{array}{c}\text { Area } \\
\%\end{array}$ \\
\hline $141.511 \mathrm{MF}$ & 1.8177 & 7566.73096 & 69.37975 & 49.5695 \\
\hline $245.245 \mathrm{FM}$ & 1.8769 & 7698.17188 & 68.35975 & 50.4305 \\
\hline
\end{tabular}


Data File D: \ChemSta...-CHIRAL \AS-3-89-9-0.9ML-60min 2019-09-03 09-28-32\relacation00003.D Sample Name: I-PROP

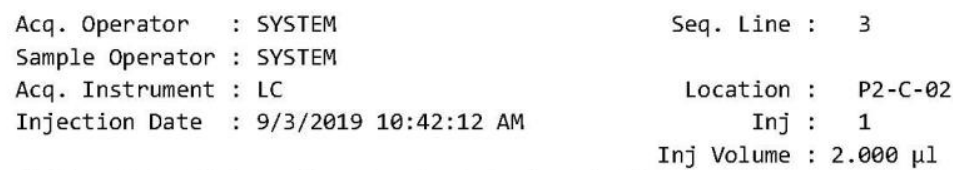

Different Inj Volume from Sample Entry! Actual Inj Volume : $4.000 \mu 1$

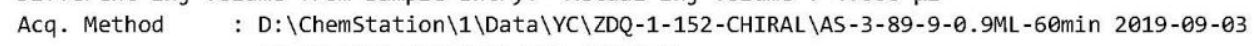
09-28-32\4-AS3-89-9-1ml-60min.M

Last changed : 9/3/2019 9:25:44 AM by SYSTEM

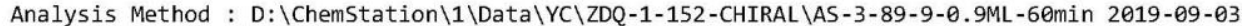
09-28-32\4-AS3-89-9-1ml-60min.M (Sequence Method)

Last changed : 11/13/2019 $8: 24: 43$ PM by SYSTEM

(modified after loading)

Additional Info : Peak(s) manually integrated

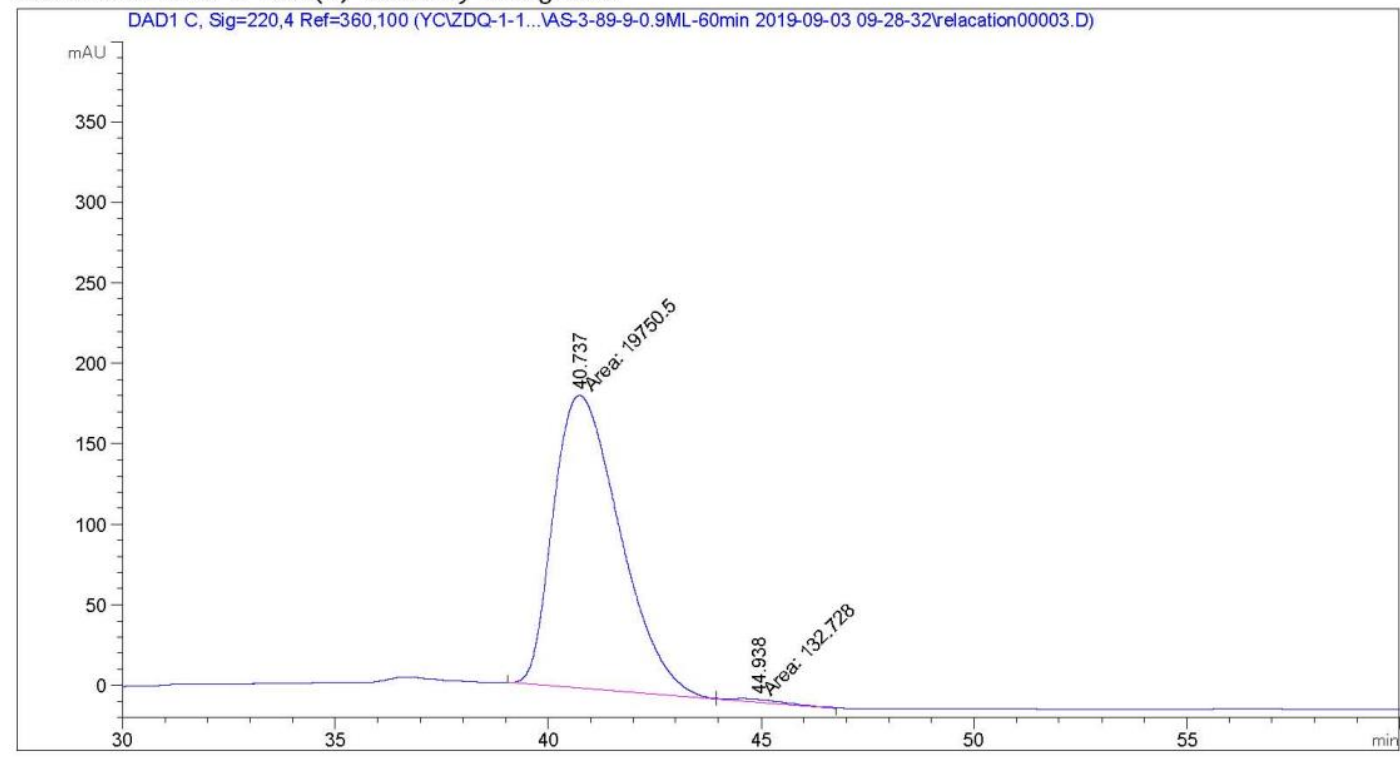

Area Percent Report

\begin{tabular}{|c|c|c|c|c|}
\hline Sorted By & : & Signal & & \\
\hline Multiplier & : & 1.0000 & & \\
\hline Dilution & : & 1.0000 & & \\
\hline \multicolumn{5}{|c|}{ Use Multiplier \& Dilution Factor with ISTDs } \\
\hline \multicolumn{5}{|c|}{ Signal 1: DAD1 C, Sig $=220,4 \operatorname{Ref}=360,100$} \\
\hline $\begin{array}{l}\text { Peak RetTime Type } \\
\# \quad \text { [min] }\end{array}$ & $\begin{array}{l}\text { Width } \\
\text { [min] }\end{array}$ & $\begin{array}{c}\text { Area } \\
{\left[\mathrm{mAU}^{*} \mathrm{~s}\right]}\end{array}$ & $\begin{array}{l}\text { Height } \\
{[\mathrm{mAU}]}\end{array}$ & $\begin{array}{c}\text { Area } \\
\%\end{array}$ \\
\hline$-\cdots|-\cdots----|$ & $-\cdots-\cdots$ & $|-\ldots-n|$ & | & $-\ldots$ \\
\hline $140.737 \mathrm{MF}$ & 1.8114 & $1.97505 \mathrm{e} 4$ & 181.72626 & 99.3325 \\
\hline $244.938 \mathrm{FM}$ & 1.3275 & 132.72816 & 1.66638 & 0.6675 \\
\hline
\end{tabular}


$\mathrm{OHC}$<smiles>CCC(C)CC(Br)(OSc1ccccc1)S(=O)(=O)O</smiles>

Data File D: \Chem32\...RAC \Bn-AS-H-85-15-1ML-80-MIN 2019-07-25 16-05-54\002-P2-A5-Bn-Rac.D Sample Name: Bn-Rac

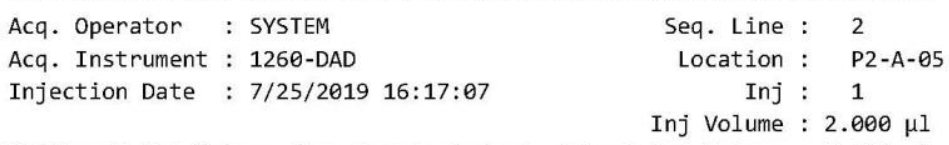

Different Inj Volume from Sample Entry! Actual Inj Volume : $3.000 \mu$

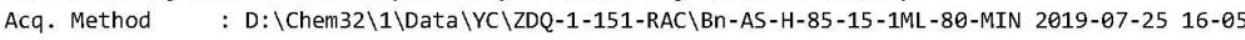
-54\ZDQ-AS-H-85-15-1ML-80MIN.M

Last changed : 7/25/2019 17:23:30 by SYSTEM (modified after loading)

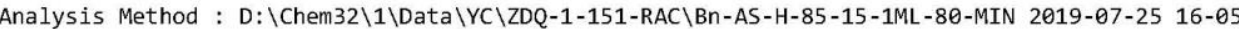
-54\ZDQ-AS-H-85-15-1ML-80MIN.M (Sequence Method)

Last changed : 11/12/2019 21:03:03 by SYSTEM

(modified after loading)

Additional Info : Peak(s) manually integrated

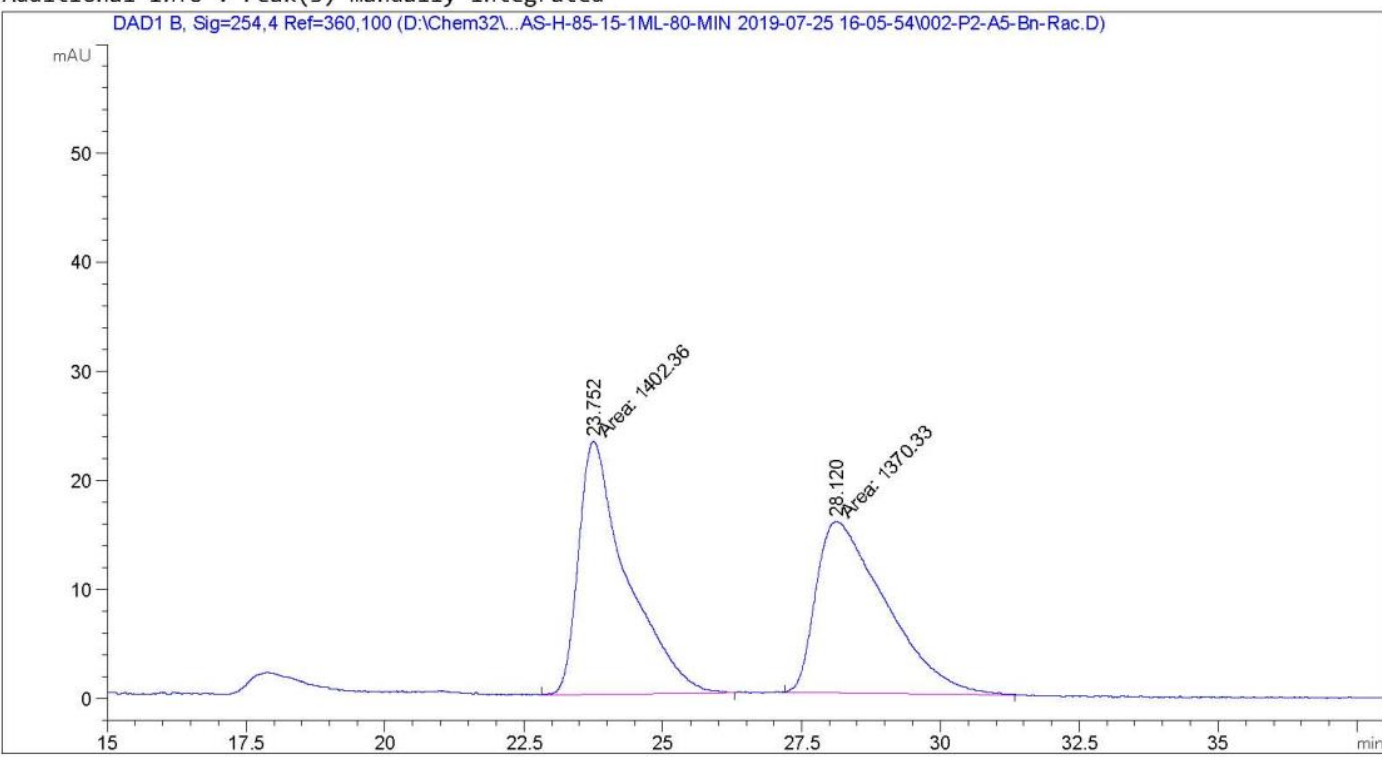

Area Percent Report

\begin{tabular}{|c|c|c|c|c|}
\hline Sorted By & : & Signal & & \\
\hline Multiplier & : & 1.0000 & & \\
\hline Dilution & : & 1.0000 & & \\
\hline \multicolumn{5}{|c|}{ Use Multiplier \& Dilution Factor with ISTDs } \\
\hline \multicolumn{5}{|c|}{ Signal 1: DAD1 B, Sig $=254,4$ Ref $=360,100$} \\
\hline $\begin{array}{l}\text { Peak RetTime Type } \\
\# \quad[\mathrm{~min}]\end{array}$ & $\begin{array}{l}\text { Width } \\
{[\text { min] }}\end{array}$ & $\begin{array}{c}\text { Area } \\
{\left[\mathrm{mAU}^{*} \mathrm{~s}\right]}\end{array}$ & $\begin{array}{l}\text { Height } \\
{[\mathrm{mAU}]}\end{array}$ & $\begin{array}{c}\text { Area } \\
\%\end{array}$ \\
\hline 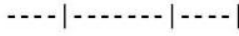 & $-\cdots-\cdots$ & $|---1-1|$ & | & $-\ldots-n$ \\
\hline $123.752 \mathrm{MM}$ & 1.0075 & 1402.36279 & 23.19860 & 50.5776 \\
\hline 228.120 MM & 1.4535 & 1370.33459 & 15.71268 & 49.4224 \\
\hline
\end{tabular}


Data File D: \CHEM32\...151-RAC \BN-AS-H-85-15-1ML-80-MIN 2019-07-25 16-05-54\003-P2-A6-Bn.D Sample Name: Bn

\begin{tabular}{|c|c|c|c|}
\hline Acq. Operator & : SYSTEM & Seq. Line & 3 \\
\hline Acq. Instrument & $: 1260-D A D$ & Location & $P 2-A-06$ \\
\hline Injection Date & : 7/25/2019 17:24:20 & $\begin{array}{r}\text { Inj } \\
\text { Inj Volume }\end{array}$ & $\begin{array}{cc}: & 1 \\
: & 2.000 \mu 1\end{array}$ \\
\hline
\end{tabular}

Different Inj Volume from Sample Entry! Actual Inj Volume : $3.000 \mu$

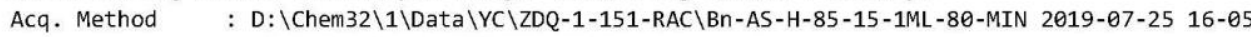
-54\ZDQ-AS-H-85-15-1ML-80MIN.M

Last changed : 7/25/2019 $17: 24: 58$ by SYSTEM

(modified after loading)

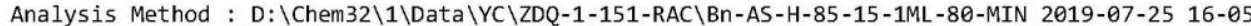

-54\ZDQ-AS-H-85-15-1ML-80MIN.M (Sequence Method)

Last changed : 11/12/2019 21:05:41 by SYSTEM

(modified after loading)

Additional Info : Peak(s) manually integrated

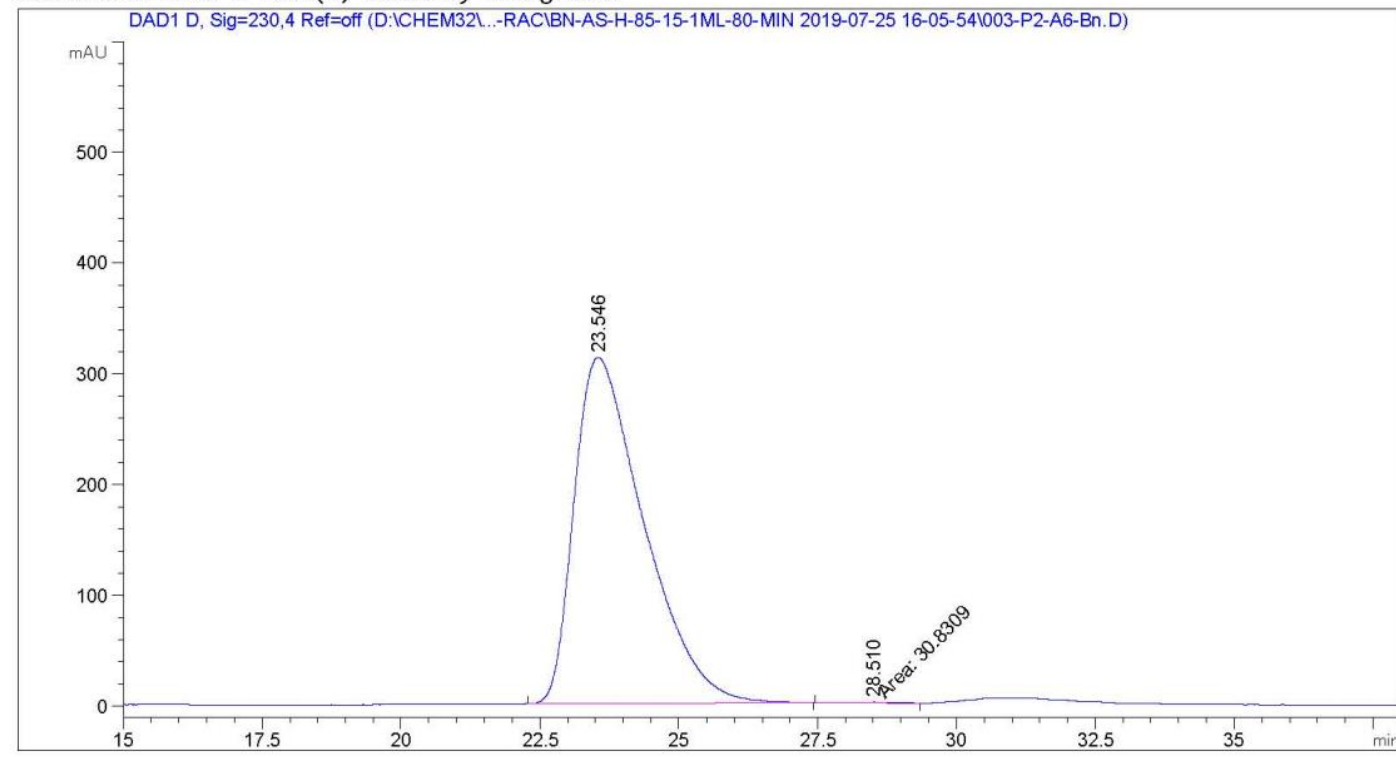

Area Percent Report

\begin{tabular}{|c|c|c|c|c|}
\hline Sorted By & : & Signal & & \\
\hline Multiplier & : & 1.0000 & & \\
\hline Dilution & : & 1.0000 & & \\
\hline \multicolumn{5}{|c|}{ Use Multiplier \& Dilution Factor with ISTDs } \\
\hline \multicolumn{5}{|c|}{ Signal 1: DAD1 D, Sig=230,4 Ref=off } \\
\hline $\begin{array}{l}\text { Peak RetTime Type } \\
\#[\text { min] }\end{array}$ & $\begin{array}{l}\text { Width } \\
\text { [min] }\end{array}$ & $\begin{array}{c}\text { Area } \\
{\left[\mathrm{mAU}^{*} \mathrm{~s}\right]}\end{array}$ & $\begin{array}{l}\text { Height } \\
{[\mathrm{mAU}]}\end{array}$ & $\begin{array}{c}\text { Area } \\
\%\end{array}$ \\
\hline$-\cdots|-\ldots-n|-\infty$ & | & - & & - \\
\hline $123.546 \mathrm{BB}$ & 1.2385 & $2.72668 \mathrm{e} 4$ & 312.34875 & 99.8871 \\
\hline 228.510 MM & 0.9786 & 30.83085 & $5.25100 \mathrm{e}-1$ & 0.1129 \\
\hline
\end{tabular}


$\mathrm{OHC} \quad \mathrm{SO}_{2} \mathrm{Ph}$

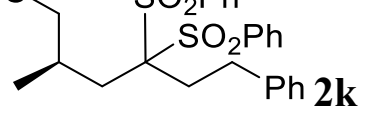

Data File D: \Chem32\...-6\AS-3-90-10-1ML-80MIN 2019-07-12 15-52-13\002-P1-C1-ZDQ-1-124-6.D Sample Name: ZDQ-1-124-6

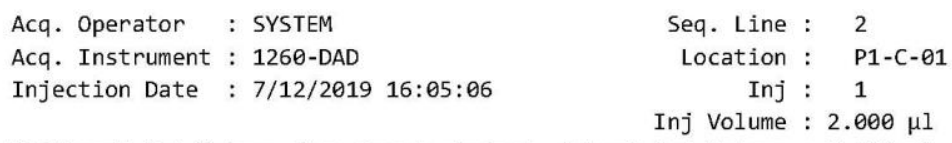

Different Inj Volume from Sample Entry! Actual Inj Volume : $3.000 \mu 1$

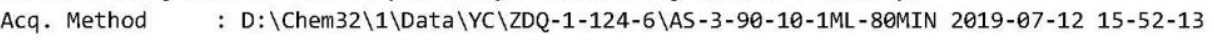
IZDQ-AS3-90-10-1ML-80MIN.M

Last changed : 7/12/2019 17:19:25 by SYSTEM (modified after loading)

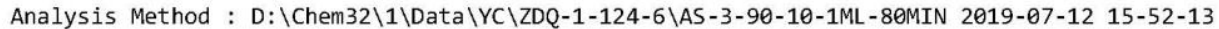
IZDQ-AS3-90-10-1ML-80MIN.M (Sequence Method)

Last changed : 11/12/2019 20:41:36 by SYSTEM

(modified after loading)

Additional Info : Peak(s) manually integrated

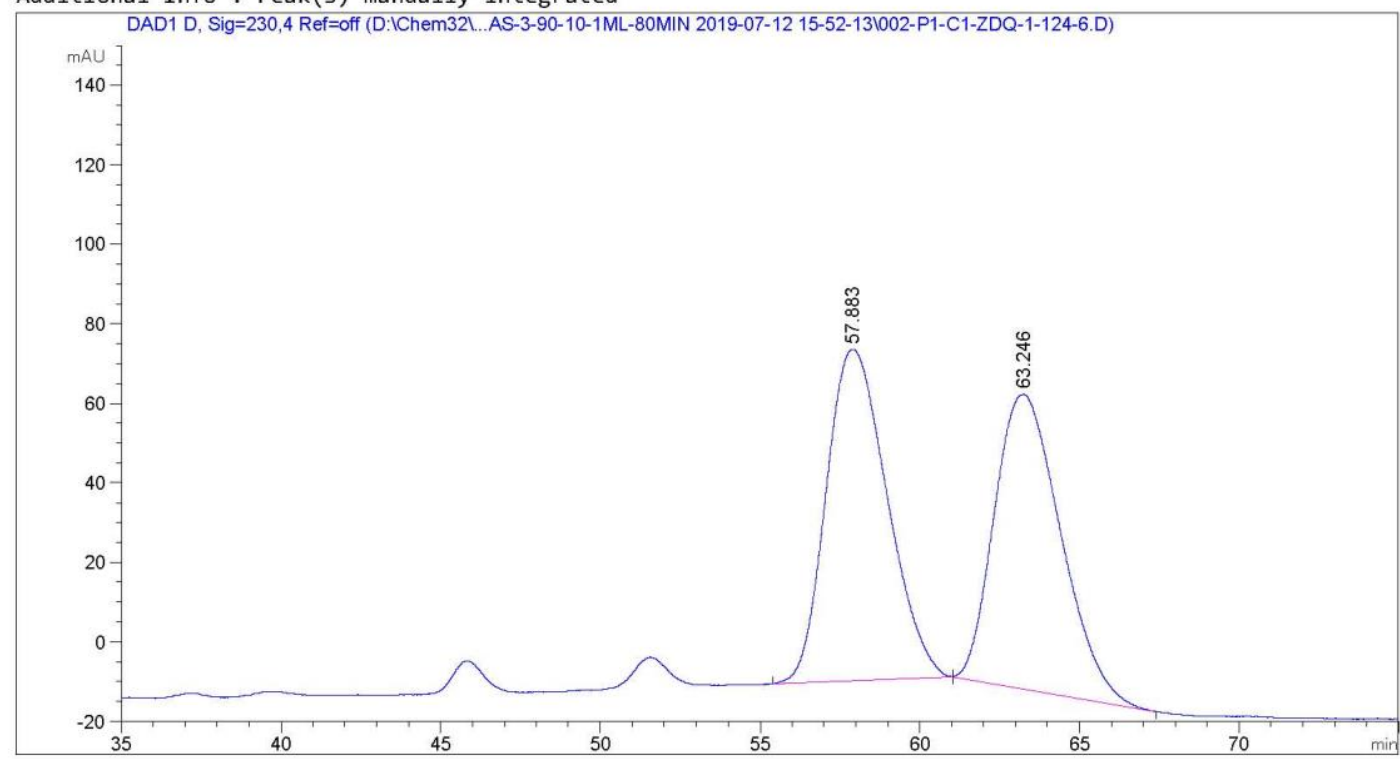

Area Percent Report

\begin{tabular}{|c|c|c|c|c|}
\hline Sorted By & : & Signal & & \\
\hline Multiplier & : & 1.0000 & & \\
\hline Dilution & : & 1.0000 & & \\
\hline \multicolumn{5}{|c|}{ Use Multiplier \& Dilution Factor with ISTDs } \\
\hline \multicolumn{5}{|c|}{ Signal 1: DAD1 D, Sig $=230,4$ Ref $=0 f f$} \\
\hline $\begin{array}{l}\text { Peak RetTime Type } \\
\# \quad[\mathrm{~min}]\end{array}$ & $\begin{array}{l}\text { Width } \\
{[\text { min] }}\end{array}$ & $\begin{array}{c}\text { Area } \\
{[\mathrm{mAU} * \mathrm{~s}]}\end{array}$ & $\begin{array}{l}\text { Height } \\
{[\mathrm{mAU}]}\end{array}$ & $\begin{array}{c}\text { Area } \\
\%\end{array}$ \\
\hline$---|-n|--n|-n|$ & $-\cdots-\cdots$ & 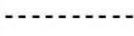 & $\mid$ & $-\cdots-1$ \\
\hline $157.883 \mathrm{BB}$ & 1.5709 & $1.10243 \mathrm{e} 4$ & 83.35960 & 50.7642 \\
\hline $263.246 \mathrm{BB}$ & 1.6980 & $1.06924 \mathrm{e} 4$ & 74.07722 & 49.2358 \\
\hline
\end{tabular}


Data File D: \Chem32\...-6\AS-3-90-10-1ML-80MIN 2019-07-12 15-52-13\003-P1-C2-ZDQ-1-122-6.D Sample Name: ZDQ-1-122-6

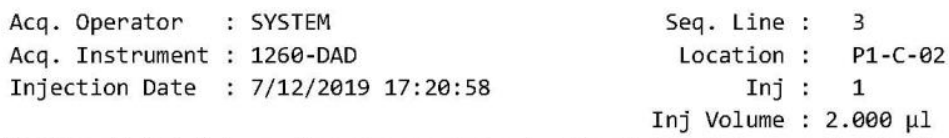

Different Inj Volume from Sample Entry! Actual Inj Volume : $3.000 \mu \mathrm{l}$

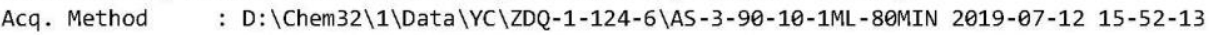
IZDQ-AS3-90-10-1ML-80MIN.M

Last changed : 7/12/2019 18:36:24 by SYSTEM (modified after loading)

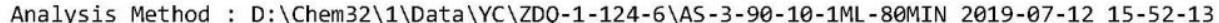
IZDQ-AS3-90-10-1ML-80MIN.M (Sequence Method)

Last changed : 11/12/2019 20:43:16 by SYSTEM

(modified after loading)

Additional Info : Peak(s) manually integrated

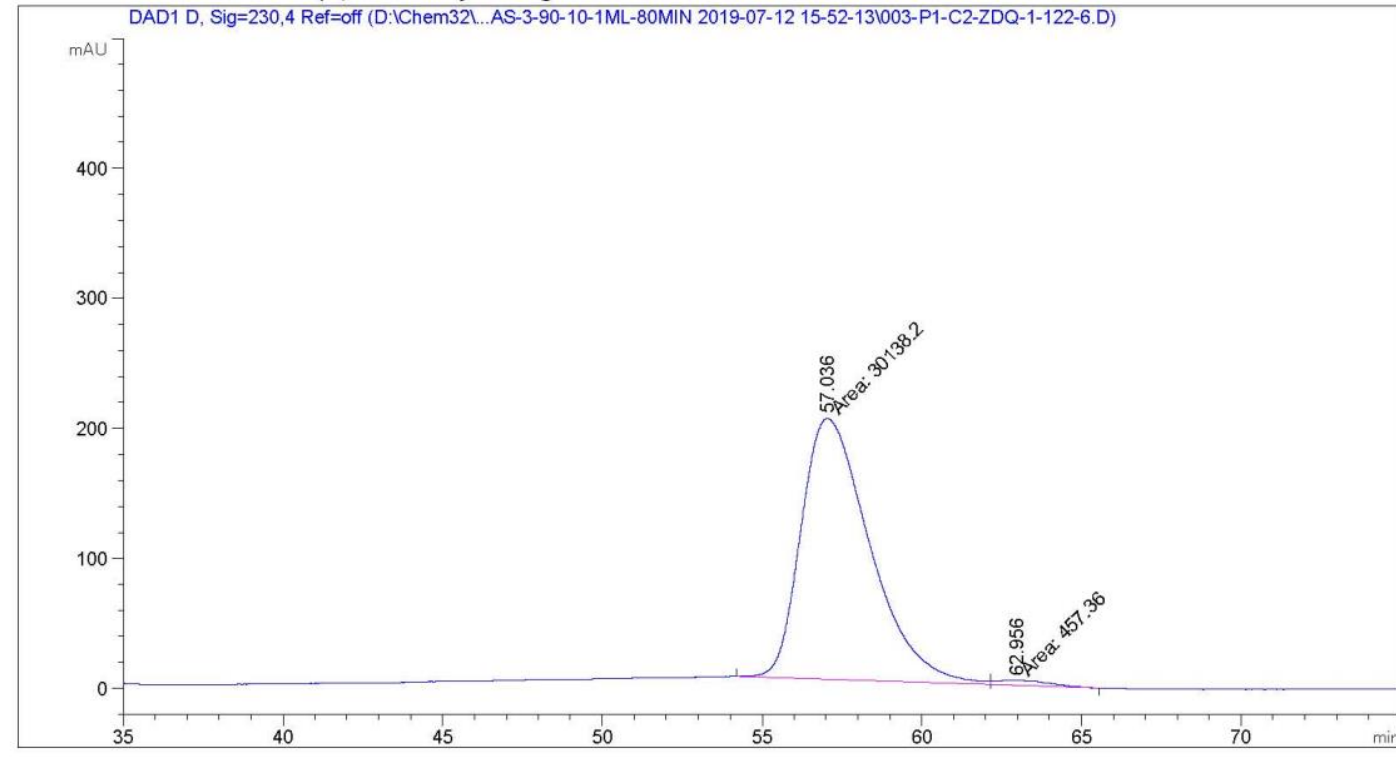

Area Percent Report

\begin{tabular}{|c|c|c|c|c|}
\hline Sorted By & : & Signal & & \\
\hline Multiplier & : & 1.0000 & & \\
\hline Dilution & : & 1.0000 & & \\
\hline \multicolumn{5}{|c|}{ Use Multiplier \& Dilution Factor with ISTDs } \\
\hline \multicolumn{5}{|c|}{ Signal 1: DAD1 D, Sig=230,4 Ref=off } \\
\hline $\begin{array}{l}\text { Peak RetTime Type } \\
\# \quad \text { [min] }\end{array}$ & $\begin{array}{l}\text { Width } \\
\text { [min] }\end{array}$ & $\begin{array}{c}\text { Area } \\
{[\mathrm{mAU} * \mathrm{~s}]}\end{array}$ & $\begin{array}{l}\text { Height } \\
{[\mathrm{mAU}]}\end{array}$ & $\begin{array}{c}\text { Area } \\
\%\end{array}$ \\
\hline$---|-------|$ & -----1 & $|-------|$ & $\mid-2-1$ & 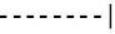 \\
\hline $157.036 \mathrm{MF}$ & 2.5007 & $3.01382 \mathrm{e} 4$ & 200.86488 & 98.5051 \\
\hline $262.956 \mathrm{FM}$ & 1.9437 & 457.36020 & 3.92176 & 1.4949 \\
\hline
\end{tabular}




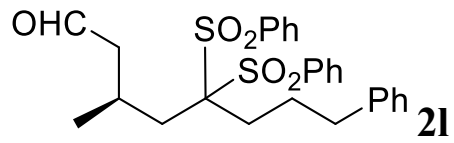

Data File D: \ChemSta...ata\YC\ZDQ-1-128\AS-85-15-1ML 2019-07-02 23-21-21 \relacatione0008.D Sample Name: ZDQ-1-129-4

\begin{tabular}{|c|c|c|c|}
\hline $\begin{array}{l}\text { Acq. Operator } \\
\text { Sample Operator }\end{array}$ & $\begin{array}{l}: \text { SYSTEM } \\
: \text { SYSTEM }\end{array}$ & Seq. Line & 8 \\
\hline Acq. Instrument & : LC & Location & $P 2-A-11$ \\
\hline Injection Date & : 7/3/2019 5:44:01 AM & $\begin{array}{r}\text { Inj } \\
\text { Inj Volume }\end{array}$ & $\begin{array}{lc}: & 1 \\
: & 2.000 \mu l\end{array}$ \\
\hline
\end{tabular}

Acq. Method : D: \ChemStation $\backslash 1 \backslash$ Data $\backslash Y C \backslash Z D Q-1-128 \backslash A S-85-15-1 M L$ 2019-07-02 23-21-21 \4-AS385-15-1ml-60min.M

Last changed : 4/9/2019 6:45:08 PM by SYSTEM

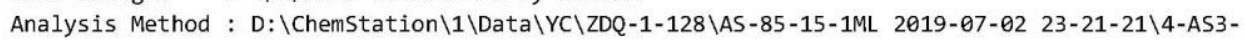
85-15-1ml-60min.M (Sequence Method)

Last changed : 11/13/2019 7:45:15 PM by SYSTEM (modified after loading)

Additional Info : Peak(s) manually integrated

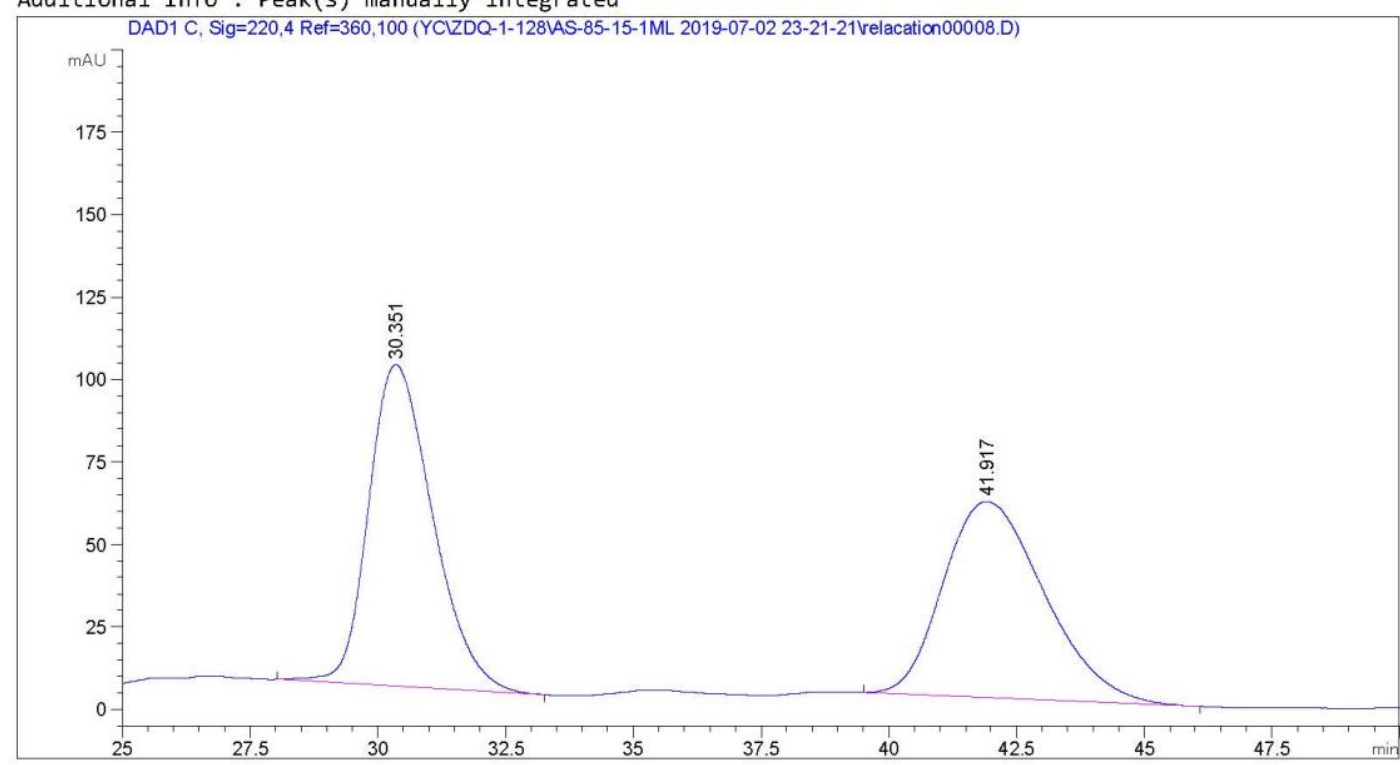

Area Percent Report

\begin{tabular}{|c|c|c|c|c|}
\hline Sorted By & : & Signal & & \\
\hline Multiplier & : & 1.0000 & & \\
\hline Dilution & : & 1.0000 & & \\
\hline \multicolumn{5}{|c|}{ Use Multiplier \& Dilution Factor with ISTDs } \\
\hline \multicolumn{5}{|c|}{ Signal 1: DAD1 C, Sig $=220,4 \operatorname{Re} f=360,100$} \\
\hline $\begin{array}{l}\text { Peak RetTime Type } \\
\#[\text { min] }\end{array}$ & $\begin{array}{l}\text { Width } \\
\text { [min] }\end{array}$ & $\begin{array}{c}\text { Area } \\
{[\mathrm{mAU} * \mathrm{~s}]}\end{array}$ & $\begin{array}{l}\text { Height } \\
{[\mathrm{mAU}]}\end{array}$ & $\begin{array}{c}\text { Area } \\
\%\end{array}$ \\
\hline$---|-n-1-n|$ & $-\ldots-1$ & $|-\ldots-2 .-1|$ & | & $-\ldots-n$ \\
\hline $130.351 \mathrm{BB}$ & 1.3409 & 8493.41309 & 97.43829 & 50.9875 \\
\hline $241.917 \mathrm{BB}$ & 1.9844 & 8164.41602 & 59.36922 & 49.0125 \\
\hline Totals : & & $1.66578 \mathrm{e} 4$ & 156.80752 & \\
\hline
\end{tabular}


Data File D: \ChemSta... ata \YC\ZDQ-1-128\AS-85-15-1ML 2019-07-02 23-21-21\relacatione0009.D Sample Name: ZDQ-1-128-4

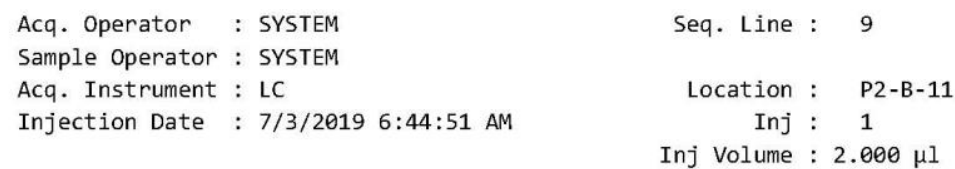

Acq. Method : D: \ChemStation $\backslash 1 \backslash$ Data $\backslash Y C \backslash Z D Q-1-128 \backslash A S-85-15-1 M L \quad 2019-07-02 \quad 23-21-21 \backslash 4-A S 3-$ 85-15-1ml-60min.M

Last changed : 4/9/2019 6:45:08 PM by SYSTEM

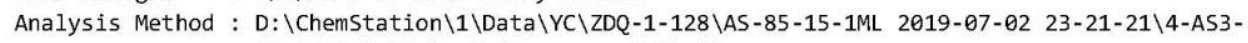
85-15-1ml-60min.M (Sequence Method)

Last changed : 11/13/2019 7:45:46 PM by SYSTEM (modified after loading)

Additional Info : Peak(s) manually integrated

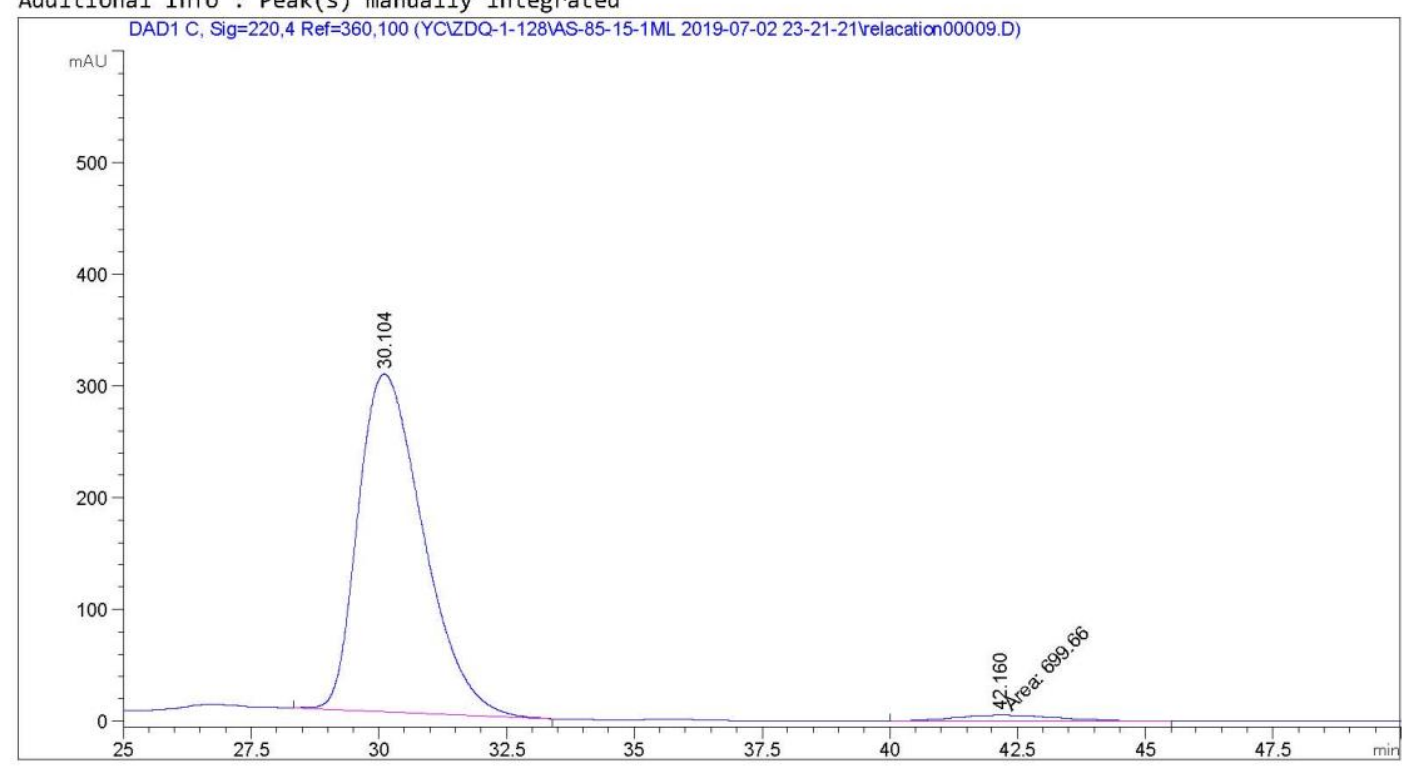

Area Percent Report

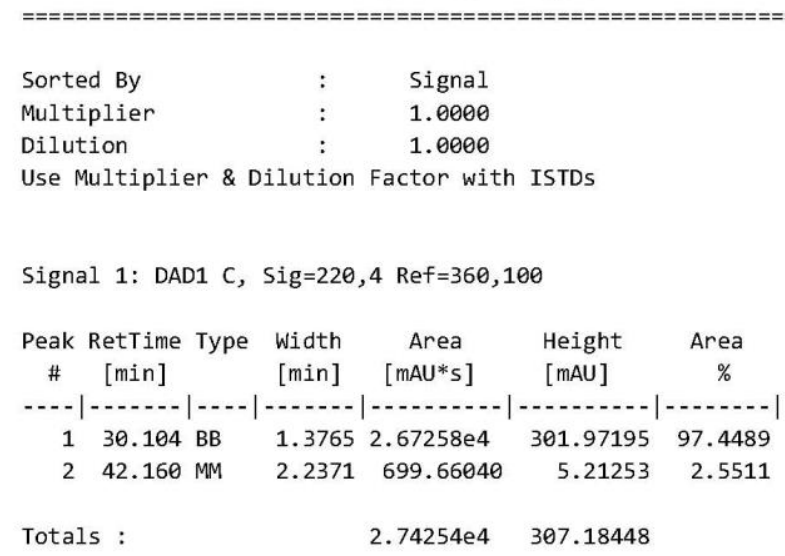

LC $11 / 13 / 2019$ 7:45:51 PM SYSTEM 


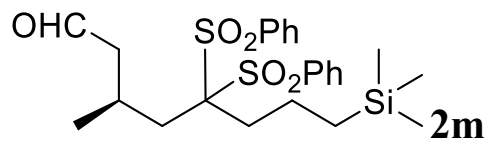

Data File D: \ChemSta...145-RAC \AS-3-91-9-0.6ML-45MIN 2019-07-22 21-29-58\relacatione0002.D Sample Name: Si-RAC

\begin{tabular}{|c|c|c|c|}
\hline $\begin{array}{l}\text { Acq. Operator } \\
\text { Sample Operator }\end{array}$ & $\begin{array}{l}\text { : SYSTEM } \\
: \text { SYSTEM }\end{array}$ & Seq. Line & 2 \\
\hline Acq. Instrument & : LC & Location & P2-A- \\
\hline Injection Date & : 7/22/2019 9:47:34 PM & $\begin{array}{r}\text { Inj } \\
\text { Inj Volume }\end{array}$ & $\begin{array}{l}1 \\
.000\end{array}$ \\
\hline
\end{tabular}

Different Inj Volume from Sample Entry! Actual Inj Volume : $3.000 \mu 1$

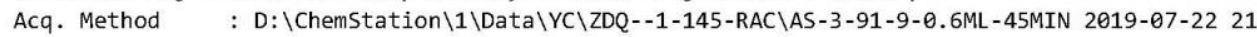
$-29-58 \backslash 4-A S 3-91-9-0.6 \mathrm{ml}-45 \mathrm{~min} . \mathrm{M}$

Last changed : 7/22/2019 9:26:36 PM by SYSTEM

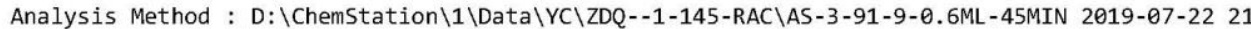

-29-58\4-AS3-91-9-0.6ml-45min.M (Sequence Method)

Last changed : 11/13/2019 7:58:33 PM by SYSTEM

(modified after loading)

Additional Info : Peak(s) manually integrated

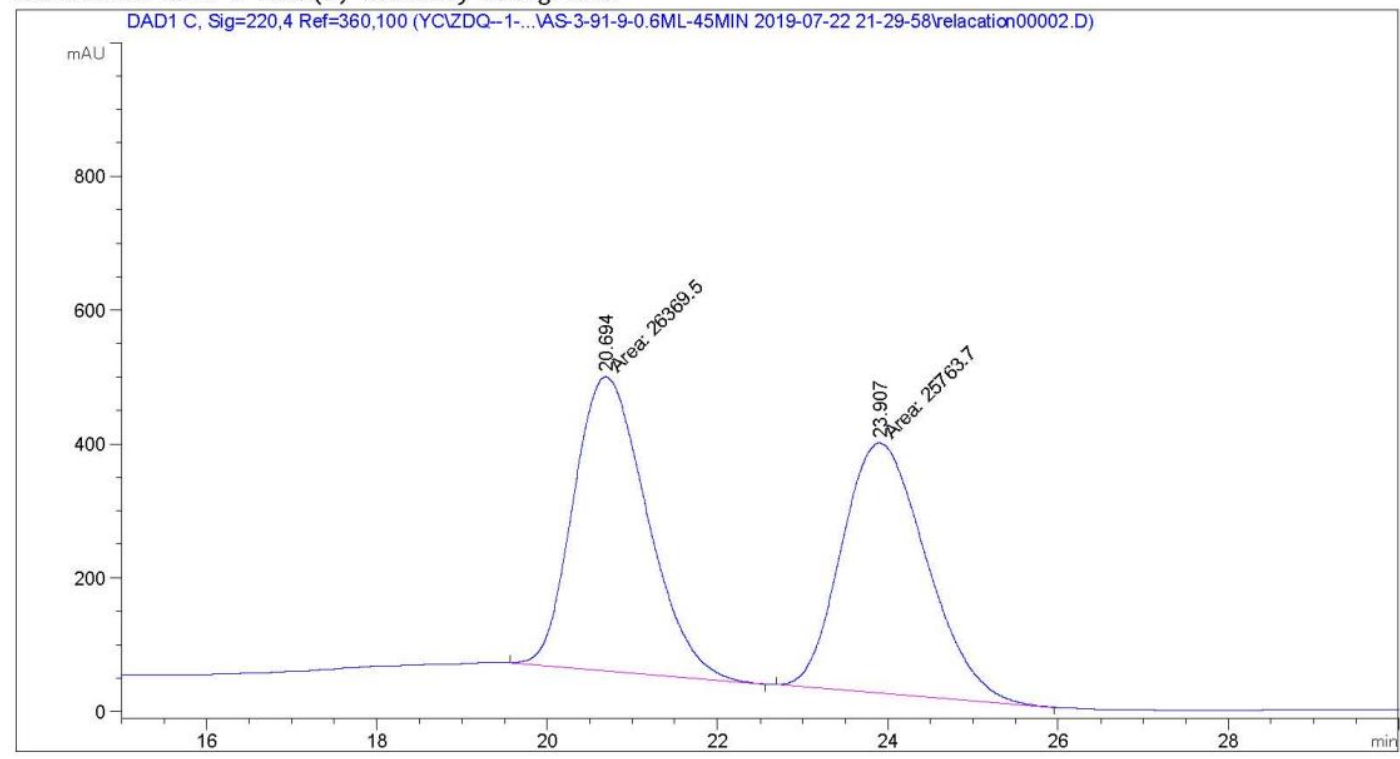

Area Percent Report

\begin{tabular}{|c|c|c|c|c|}
\hline Sorted By & $:$ & Signal & & \\
\hline Multiplier & : & 1.0000 & & \\
\hline Dilution & : & 1.0000 & & \\
\hline \multicolumn{5}{|c|}{ Use Multiplier \& Dilution Factor with ISTDs } \\
\hline \multicolumn{5}{|c|}{ Signal 1: DAD1 C, Sig=220,4 Ref $=360,100$} \\
\hline $\begin{array}{l}\text { Peak RetTime Type } \\
\# \quad[\min ]\end{array}$ & $\begin{array}{l}\text { Width } \\
\text { [min] }\end{array}$ & $\begin{array}{c}\text { Area } \\
{[\mathrm{mAU} * \mathrm{~s}]}\end{array}$ & $\begin{array}{l}\text { Height } \\
{[\mathrm{mAU}]}\end{array}$ & $\begin{array}{c}\text { Area } \\
\%\end{array}$ \\
\hline 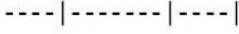 & $\mid-\cdots+$ & | & $\mid$ & $-\cdots$ \\
\hline $120.694 \mathrm{MM}$ & 1.0004 & $2.63695 \mathrm{e} 4$ & 439.31805 & 50.5810 \\
\hline 223.907 MM & 1.1497 & $2.57637 \mathrm{e} 4$ & 373.47253 & 49.4190 \\
\hline
\end{tabular}


Data File D: \ChemSta...145-RAC \AS-3-91-9-0.6ML-45MIN 2019-07-22 21-29-58\relacation00003.D Sample Name: Si
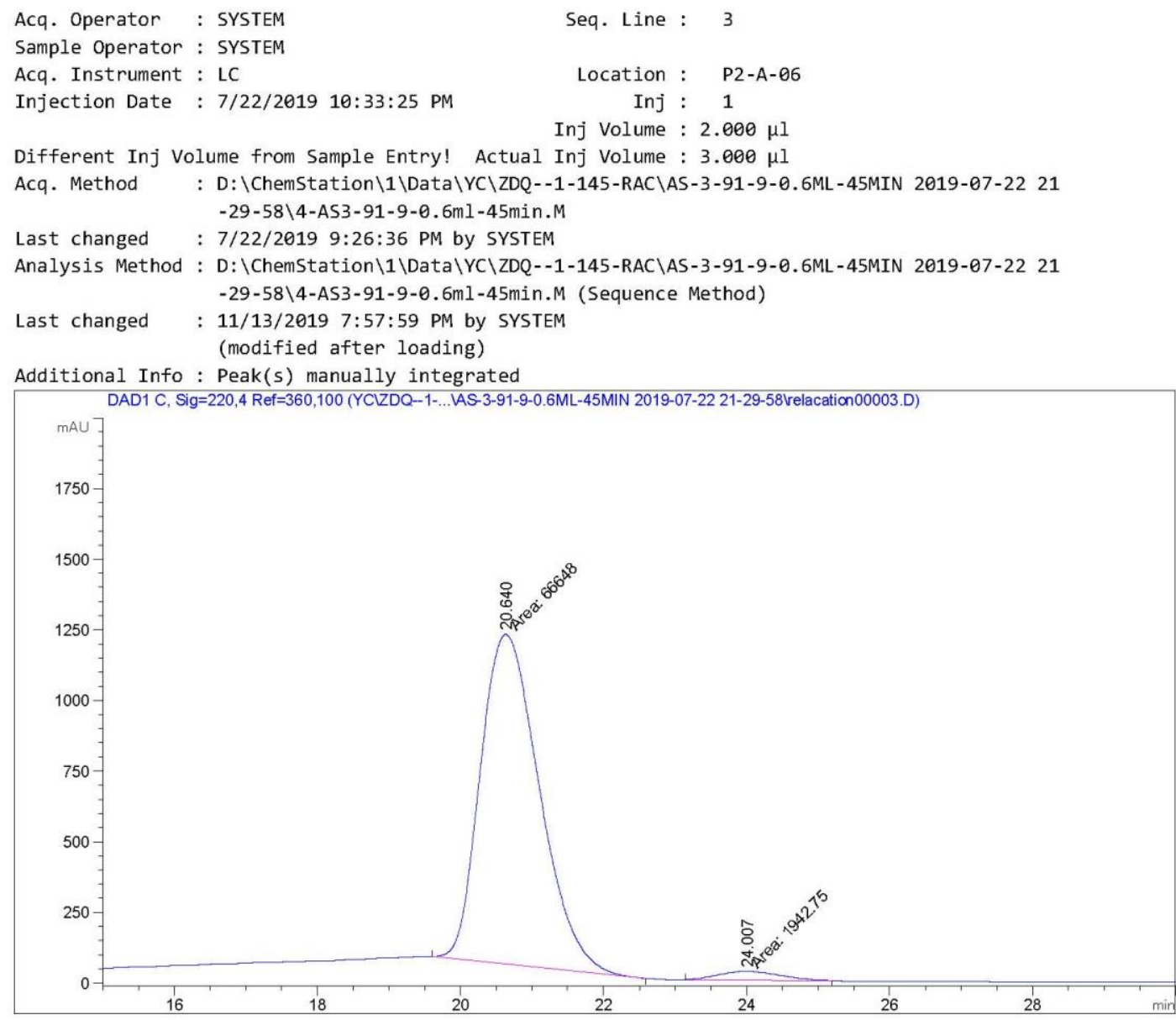

Area Percent Report

\begin{tabular}{|c|c|c|c|c|}
\hline Sorted By & : & Signal & & \\
\hline Multiplier & : & 1.0000 & & \\
\hline Dilution & : & 1.0000 & & \\
\hline \multicolumn{5}{|c|}{ Use Multiplier \& Dilution Factor with ISTDs } \\
\hline \multicolumn{5}{|c|}{ Signal 1: DAD1 C, Sig $=220,4 \operatorname{Re} f=360,100$} \\
\hline $\begin{array}{l}\text { Peak RetTime Type } \\
\#[\text { min] }\end{array}$ & $\begin{array}{l}\text { Width } \\
\text { [min] }\end{array}$ & $\begin{array}{c}\text { Area } \\
{\left[\mathrm{mAU}^{*} \mathrm{~s}\right]}\end{array}$ & $\begin{array}{l}\text { Height } \\
{[\mathrm{mAU}]}\end{array}$ & $\begin{array}{c}\text { Area } \\
\%\end{array}$ \\
\hline$-\cdots|-\ldots-n| \cdots$ & | & 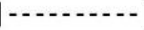 & $|-\cdots-\cdots|$ & - \\
\hline $120.640 \mathrm{MM}$ & 0.9522 & $6.66480 \mathrm{e} 4$ & 1166.58154 & 97.1676 \\
\hline 224.007 MM & 1.0284 & 1942.75269 & 31.48399 & 2.8324 \\
\hline
\end{tabular}


$\mathrm{OHC} \quad \mathrm{SO}_{2} \mathrm{Ph}$

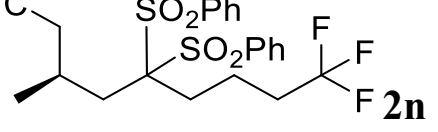

Data File D: \ChemSta...ata \YC\ZDQ-1-128\AS-85-15-1ML 2019-07-02 23-21-21 \relacatione0006.D Sample Name: ZDQ-1-129-3

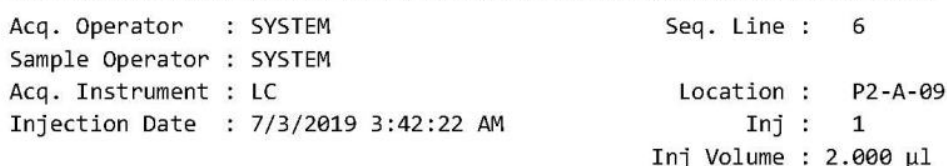

Acq. Method : D: \ChemStation $\backslash 1 \backslash D a t a \backslash Y C \backslash Z D Q-1-128 \backslash A S-85-15-1 M L \quad 2019-07-02 \quad 23-21-21 \backslash 4-A S 3-$ 85-15-1ml-60min.M

Last changed : 4/9/2019 6:45:08 PM by SYSTEM

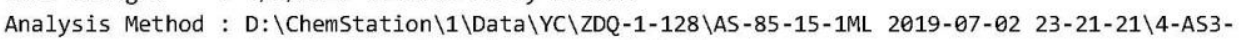
85-15-1ml-60min.M (Sequence Method)

Last changed : 11/13/2019 7:40:07 PM by SYSTEM (modified after loading)

Additional Info : Peak(s) manually integrated

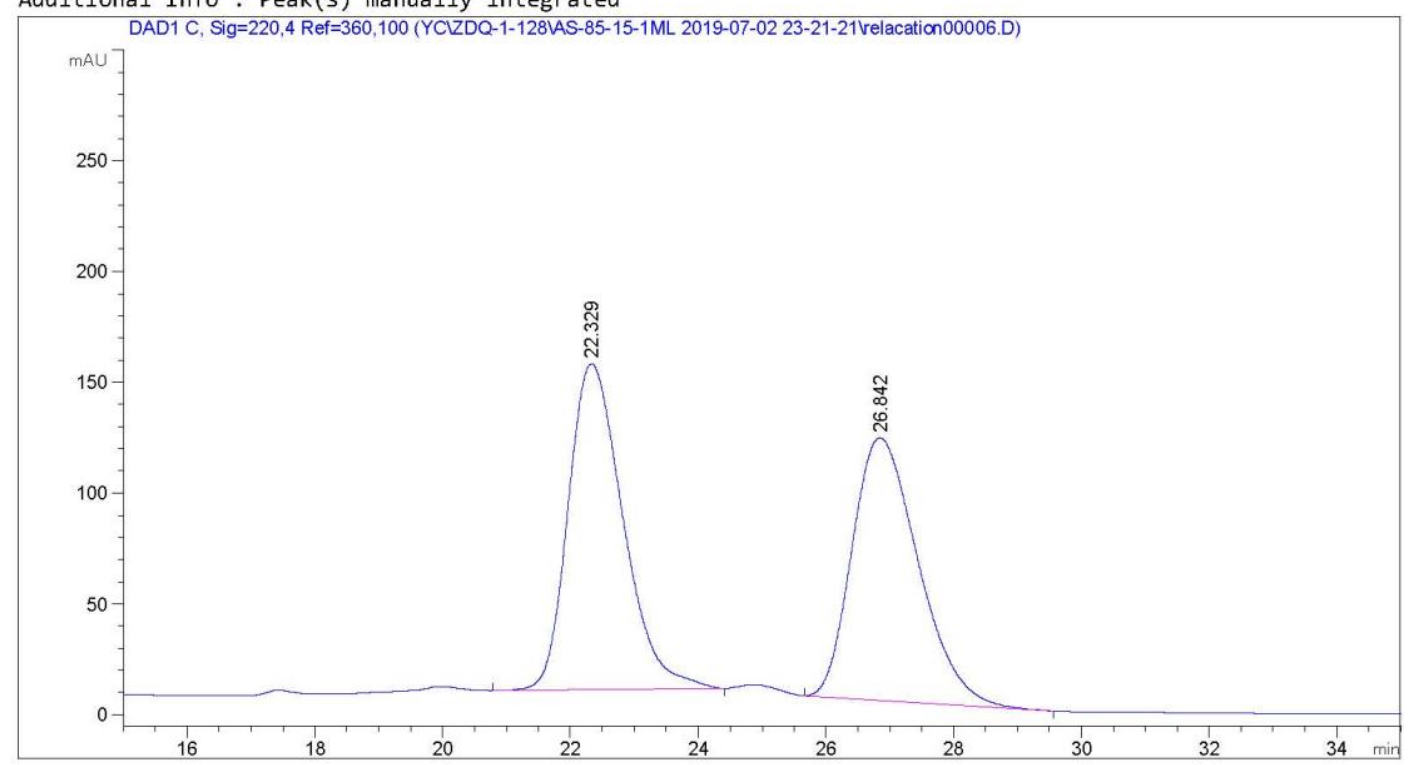

Area Percent Report

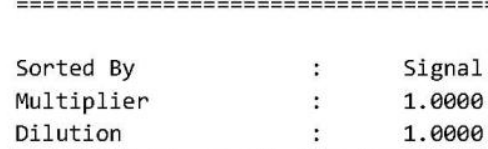

Use Multiplier \& Dilution Factor with ISTDs

Signal 1: DAD1 C, Sig $=220,4 \operatorname{Re} f=360,100$

\begin{tabular}{|c|c|c|c|c|c|c|}
\hline $\begin{array}{c}\text { Peak } \\
\#\end{array}$ & $\begin{array}{c}\text { RetTime } \\
\text { [min] }\end{array}$ & Type & $\begin{array}{l}\text { Width } \\
\text { [min] }\end{array}$ & $\begin{array}{c}\text { Area } \\
{\left[\mathrm{mAU}^{*} \mathrm{~s}\right]}\end{array}$ & $\begin{array}{l}\text { Height } \\
\text { [mAU] }\end{array}$ & $\begin{array}{c}\text { Area } \\
\%\end{array}$ \\
\hline$\ldots$ & & & & - & & - ne. \\
\hline 1 & 22.329 & BB & 0.9223 & 8749.41992 & 147.02393 & 50.7043 \\
\hline 2 & 26.842 & BB & 1.1118 & 8506.34473 & 118.44688 & 49.2957 \\
\hline
\end{tabular}

Totals : $\quad 1.72558 \mathrm{e} 4 \quad 265.47080$ 
Data File D: \ChemSta... ata \YC\ZDQ-1-128\AS-85-15-1ML 2019-07-02 23-21-21 \relacatione0007.D Sample Name: ZDQ-1-128-3
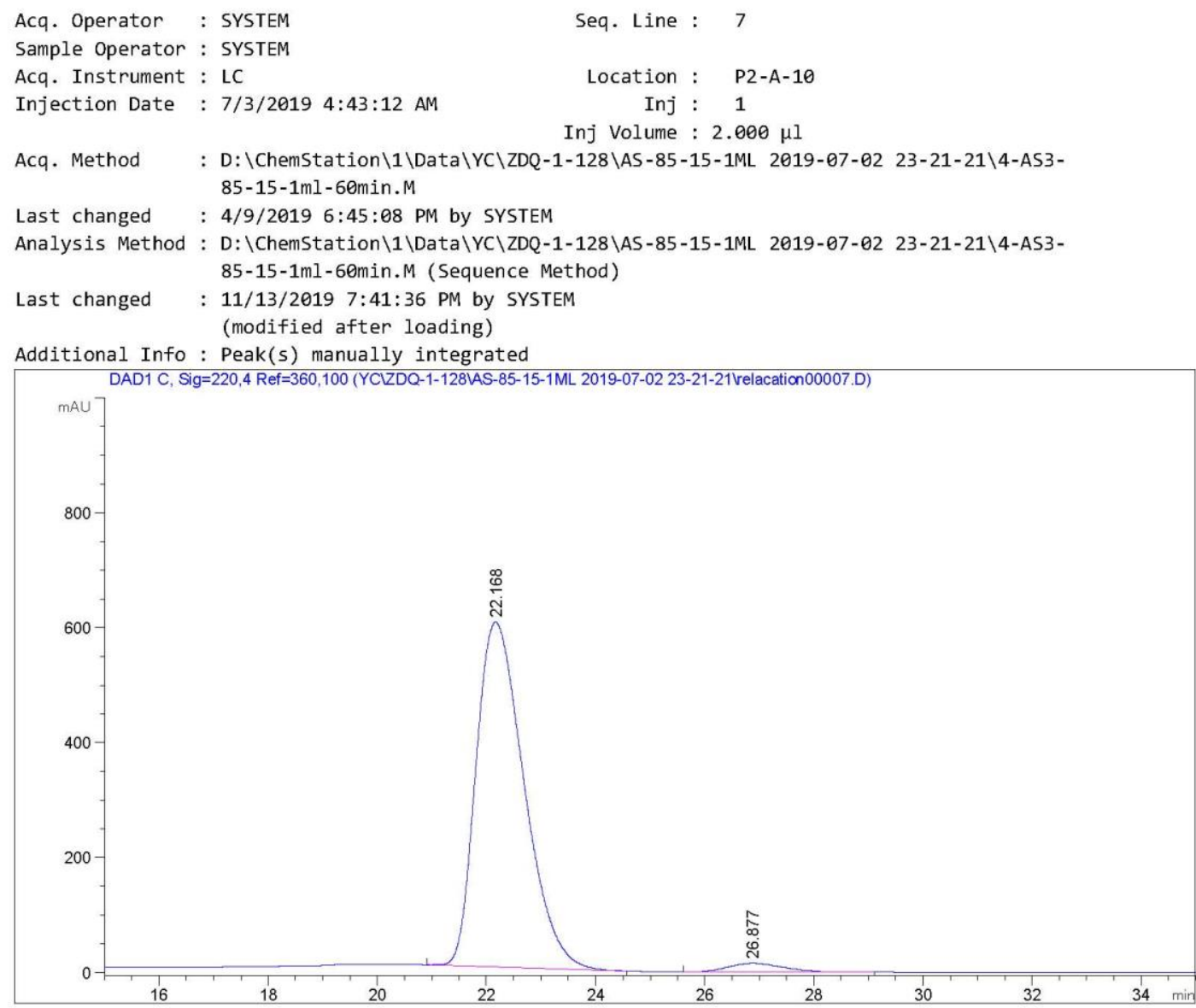

Area Percent Report

\begin{tabular}{|c|c|c|c|c|}
\hline Sorted By & : & Signal & & \\
\hline Multiplier & : & 1.0000 & & \\
\hline Dilution & : & 1.0000 & & \\
\hline \multicolumn{5}{|c|}{ Use Multiplier \& Dilution Factor with ISTDs } \\
\hline \multicolumn{5}{|c|}{ Signal 1: DAD1 C, Sig=220,4 Ref $=360,100$} \\
\hline $\begin{array}{l}\text { Peak RetTime Type } \\
\# \quad[\text { min }]\end{array}$ & $\begin{array}{l}\text { Width } \\
\text { [min] }\end{array}$ & $\begin{array}{c}\text { Area } \\
{[\mathrm{mAU} * \mathrm{~s}]}\end{array}$ & $\begin{array}{l}\text { Height } \\
\text { [mAU] }\end{array}$ & $\begin{array}{c}\text { Area } \\
\%\end{array}$ \\
\hline 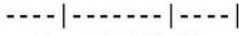 & $-\cdots-1$ & $|--\cdots+--n|$ & 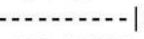 & 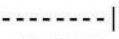 \\
\hline 122.168 BB & 0.9531 & $3.65989 \mathrm{e} 4$ & 600.44415 & 97.1663 \\
\hline $226.877 \mathrm{BB}$ & 1.0209 & 1067.33594 & 15.05914 & 2.8337 \\
\hline Totals : & & $3.76662 \mathrm{e} 4$ & 615.50330 & \\
\hline
\end{tabular}

LC 11/13/2019 7:41:41 PM SYSTEM 
$\mathrm{OHC} \quad \mathrm{SO}_{2} \mathrm{Ph}$

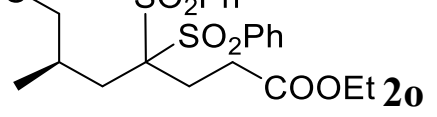

Data File D: \ChemSta...20190927-AS-3-75-25-1ml-50MIN 2019-09-27 13-35-19\relacatione0002.D Sample Name: RAC-COOET

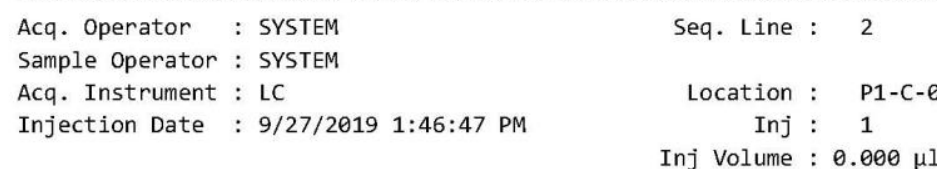

Different Inj Volume from Sample Entry! Actual Inj Volume : $3.000 \mu 1$

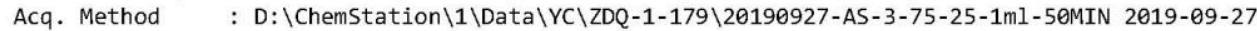
13-35-19\4-AS3-75-25-1ml-50min.M

Last changed : 9/27/2019 1:34:00 PM by SYSTEM

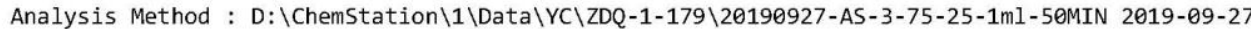

13-35-19 4 -AS3-75-25-1ml-50min.M (Sequence Method)

Last changed : 11/13/2019 8:28:14 PM by SYSTEM

(modified after loading)

Additional Info : Peak(s) manually integrated

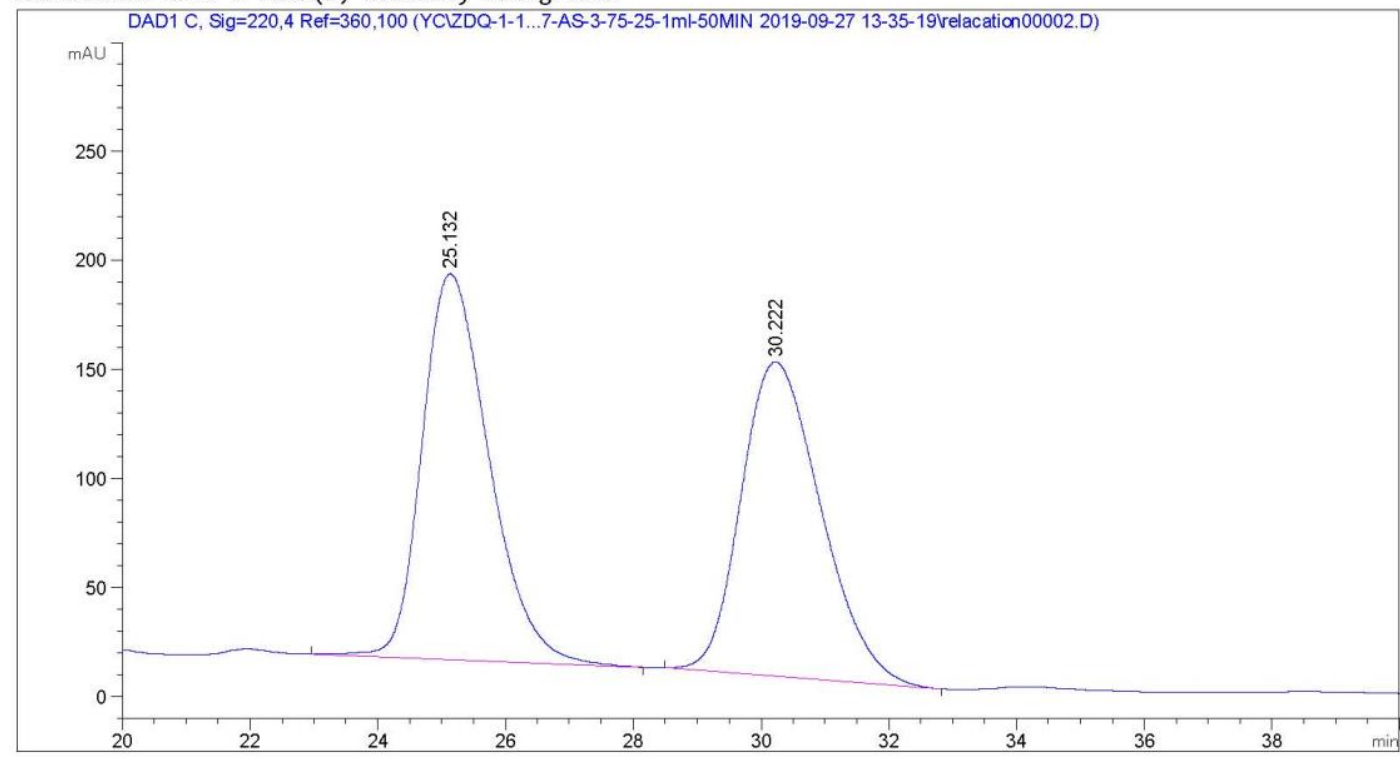

Area Percent Report

\begin{tabular}{|c|c|c|c|c|}
\hline Sorted By & : & Signal & & \\
\hline Multiplier & : & 1.0000 & & \\
\hline Dilution & : & 1.0000 & & \\
\hline \multicolumn{5}{|c|}{ Use Multiplier \& Dilution Factor with ISTDs } \\
\hline \multicolumn{5}{|c|}{ Signal 1: DAD1 C, Sig $=220,4$ Ref $=360,100$} \\
\hline $\begin{array}{l}\text { Peak RetTime Type } \\
\# \quad[\text { min] }\end{array}$ & $\begin{array}{l}\text { Width } \\
\text { [min] }\end{array}$ & $\begin{array}{c}\text { Area } \\
{\left[\mathrm{mAU}^{*} \mathrm{~s}\right]}\end{array}$ & $\begin{array}{l}\text { Height } \\
{[\mathrm{mAU}]}\end{array}$ & $\begin{array}{c}\text { Area } \\
\%\end{array}$ \\
\hline$-\cdots|-\cdots----| \mid$ & $-\cdots-1$ & | - n-nen & | & $-\cdots-1$ \\
\hline 125.132 BB & 1.1162 & $1.28589 \mathrm{e} 4$ & 176.86415 & 50.7007 \\
\hline $230.222 \mathrm{BB}$ & 1.3464 & $1.25035 \mathrm{e} 4$ & 144.09021 & 49.2993 \\
\hline
\end{tabular}


Data File D: \ChemSta...20190927-AS-3-75-25-1ml-50MIN 2019-09-27 13-35-19\relacation00003.D Sample Name: COOET
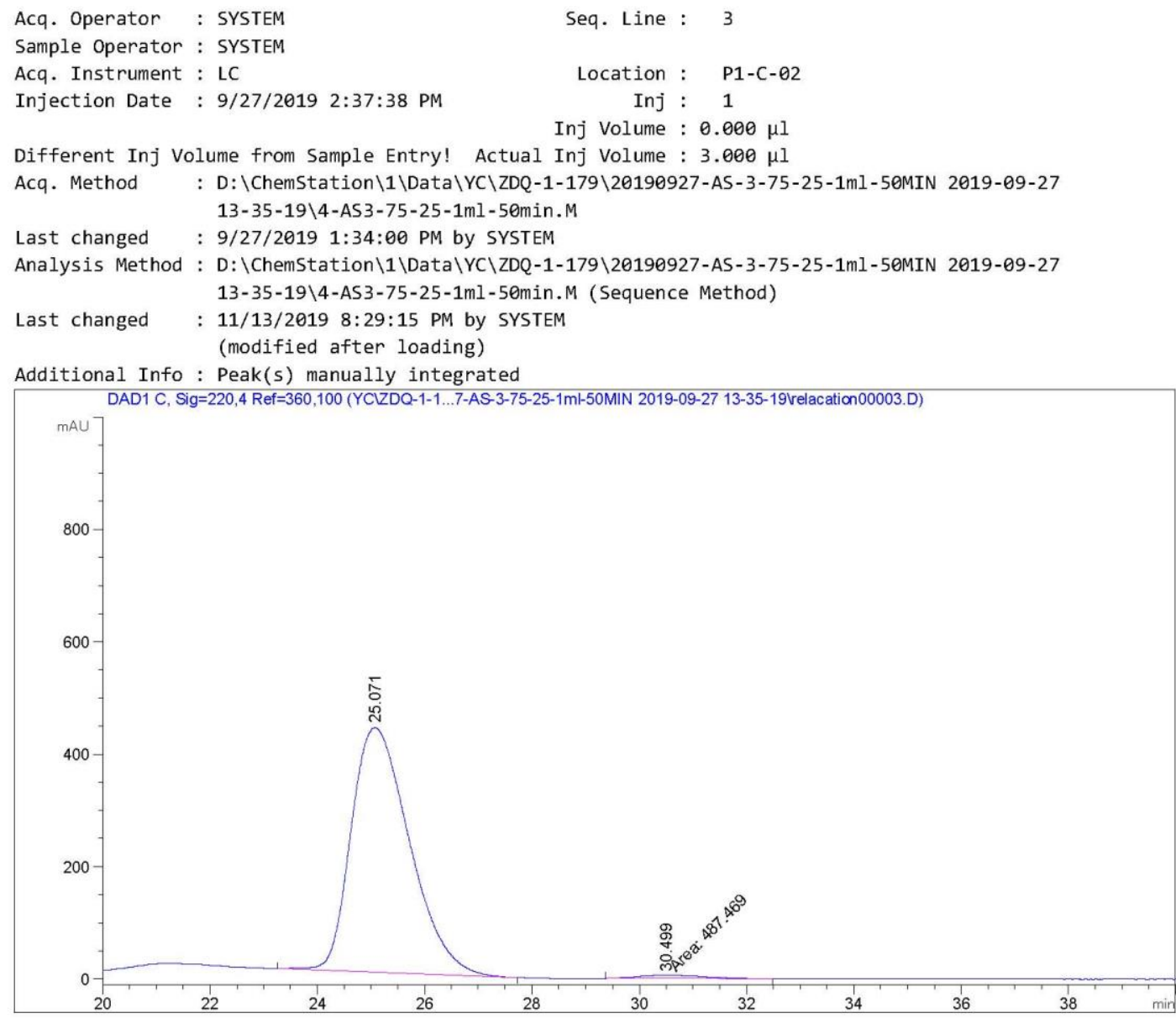

Area Percent Report

\begin{tabular}{|c|c|c|c|c|}
\hline Sorted By & : & Signal & & \\
\hline Multiplier & : & 1.0000 & & \\
\hline Dilution & : & 1.0000 & & \\
\hline \multicolumn{5}{|c|}{ Use Multiplier \& Dilution Factor with ISTDs } \\
\hline \multicolumn{5}{|c|}{ Signal 1: DAD1 C, Sig $=220,4 \operatorname{Re} f=360,100$} \\
\hline $\begin{array}{l}\text { Peak RetTime Type } \\
\# \quad \text { [min] }\end{array}$ & $\begin{array}{l}\text { Width } \\
\text { [min] }\end{array}$ & $\begin{array}{c}\text { Area } \\
{[\mathrm{mAU} * \mathrm{~s}]}\end{array}$ & $\begin{array}{l}\text { Height } \\
{[\mathrm{mAU}]}\end{array}$ & $\begin{array}{c}\text { Area } \\
\%\end{array}$ \\
\hline$---|-----|--\mid$ & $-\cdots--1$ & $|---\cdots|$ & $-\cdots-1$ & $-\ldots$ \\
\hline $125.071 \mathrm{BB}$ & 1.1813 & $3.27589 \mathrm{e} 4$ & 434.64319 & 98.5338 \\
\hline 230.499 MM & 1.3368 & 487.46890 & 6.07760 & 1.4662 \\
\hline
\end{tabular}




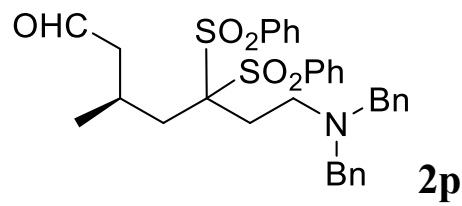

Data File D: \Chem32\...20190928-AS-H-90-10-1ML-60MIN 2019-09-28 14-47-37\002-P2-D2-RAC-N.D Sample Name: RAC-N

\begin{tabular}{|c|c|c|c|}
\hline Acq. Operator & : SYSTEM & Seq. Line : & 2 \\
\hline Acq. Instrument & : 1260-DAD & Location : & $P 2-D-\theta$ \\
\hline Injection Date & : 9/28/2019 14:56:52 & Inj & 1 \\
\hline
\end{tabular}

Different Inj Volume from Sample Entry! Actual Inj Volume : $3.000 \mu 1$

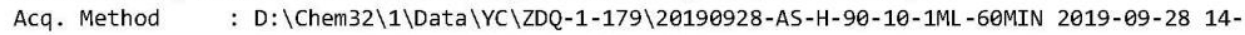
47-37\ZDQ-AS-H-90-10-1ML - 60MIN. M

Last changed : 9/28/2019 14:58:00 by SYSTEM (modified after loading)

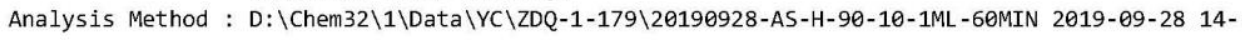
47-37\ZDQ-AS-H-90-10-1ML-60MIN.M (Sequence Method)

Last changed $\quad: 11 / 12 / 201921: 18: 59$ by SYSTEM (modified after loading)

Additional Info : Peak(s) manually integrated

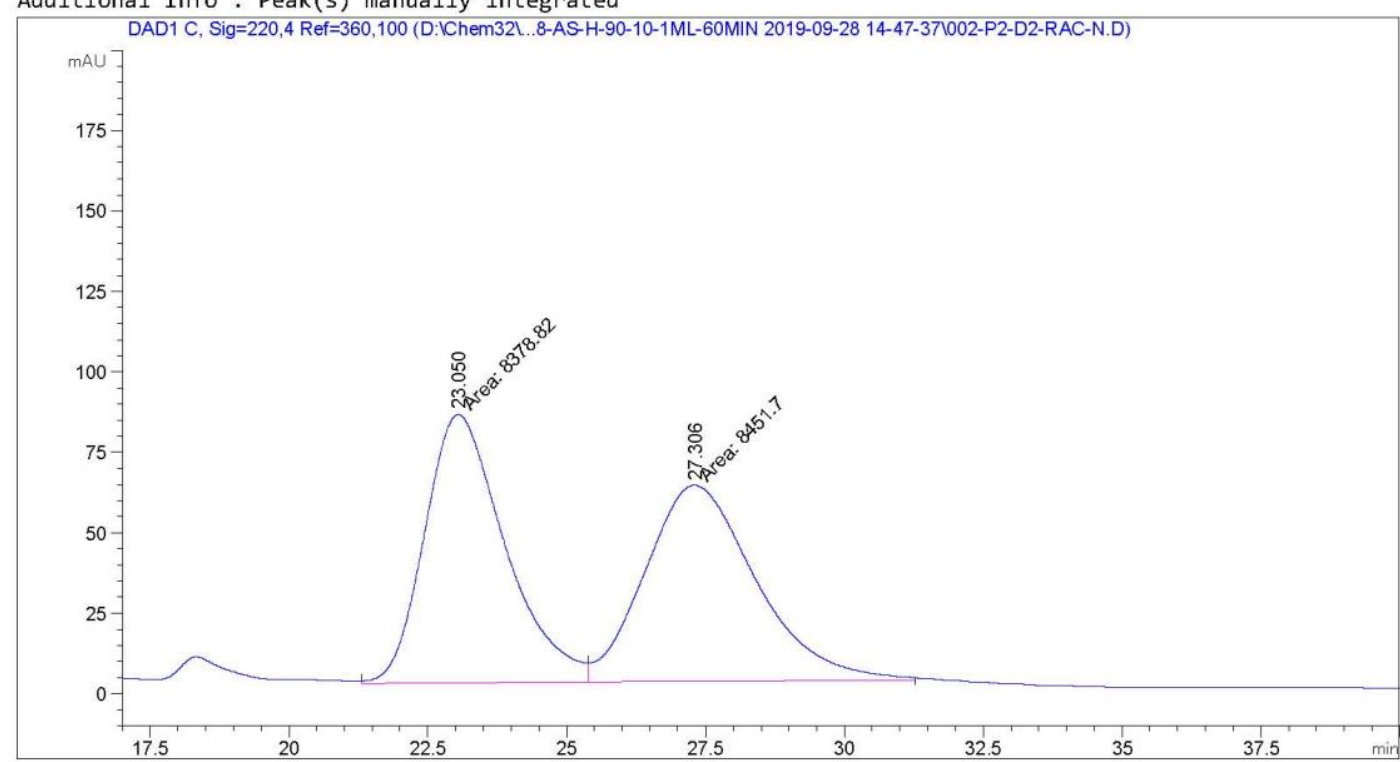

Area Percent Report

\begin{tabular}{|c|c|c|c|c|}
\hline Sorted By & : & Signal & & \\
\hline Multiplier & : & 1.0000 & & \\
\hline Dilution & : & 1.0000 & & \\
\hline \multicolumn{5}{|c|}{ Use Multiplier \& Dilution Factor with ISTDs } \\
\hline \multicolumn{5}{|c|}{ Signal 1: DAD1 C, Sig=220,4 $\operatorname{Re} f=360,100$} \\
\hline $\begin{array}{l}\text { Peak RetTime Type } \\
\# \quad[\text { min] }\end{array}$ & $\begin{array}{l}\text { Width } \\
\text { [min] }\end{array}$ & $\begin{array}{c}\text { Area } \\
{\left[\mathrm{mAU}^{*} \mathrm{~s}\right]}\end{array}$ & $\begin{array}{l}\text { Height } \\
{[\mathrm{mAU}]}\end{array}$ & $\begin{array}{c}\text { Area } \\
\%\end{array}$ \\
\hline$---|-----|--\mid$ & $-\cdots--1$ & $|---n|$ & -1 & $-\cdots-1$ \\
\hline $123.050 \mathrm{MF}$ & 1.6736 & 8378.82422 & 83.44305 & 49.7835 \\
\hline $227.306 \mathrm{FM}$ & 2.3098 & 8451.69629 & 60.98455 & 50.2165 \\
\hline
\end{tabular}


Data File D: \Chem32\...179\20190928-AS-H-90-10-1ML-60MIN 2019-09-28 14-47-37\003-P2-D3-N.D Sample Name: $\mathrm{N}$

\begin{tabular}{|c|c|c|c|}
\hline Acq. Operator & : SYSTEM & Seq. Line : & 3 \\
\hline Acq. Instrument & : 1260-DAD & Location : & P2-D-03 \\
\hline Injection Date & : 9/28/2019 15:42:43 & $\begin{array}{r}\text { Inj : } \\
\text { Inj Volume : }\end{array}$ & $\begin{array}{lc}: & 1 \\
: & 2.000 \mu 1\end{array}$ \\
\hline
\end{tabular}

Different Inj Volume from Sample Entry! Actual Inj Volume : $3.000 \mu$

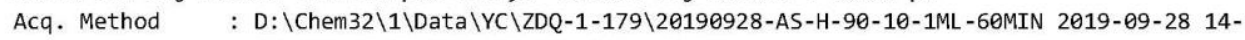

47-37\ZDQ-AS-H-90-10-1ML-60MIN.M

Last changed : 9/28/2019 $14: 58: 00$ by SYSTEM

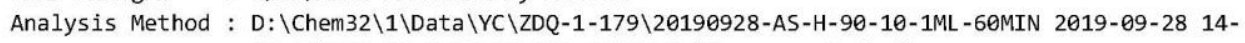
47-37\ZDQ-AS-H-90-10-1ML-60MIN.M (Sequence Method)

Last changed : 11/12/2019 21:19:55 by SYSTEM (modified after loading)

Additional Info : Peak(s) manually integrated

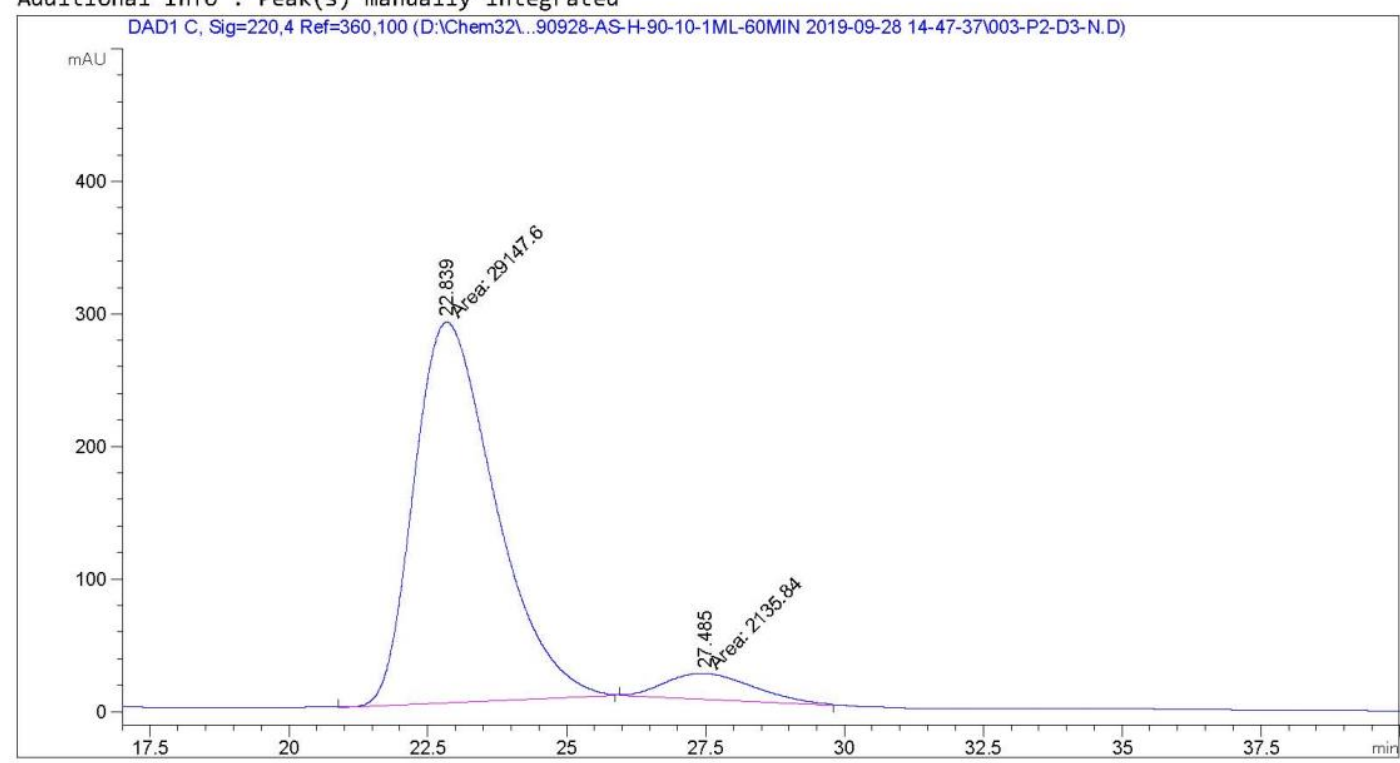

Area Percent Report

\begin{tabular}{|c|c|c|c|c|}
\hline Sorted By & : & Signal & & \\
\hline Multiplier & : & 1.0000 & & \\
\hline Dilution & : & 1.0000 & & \\
\hline \multicolumn{5}{|c|}{ Use Multiplier \& Dilution Factor with ISTDs } \\
\hline \multicolumn{5}{|c|}{ Signal 1: DAD1 C, Sig $=220,4 \operatorname{Re} f=360,100$} \\
\hline $\begin{array}{l}\text { Peak RetTime Type } \\
\# \quad[\mathrm{~min}]\end{array}$ & $\begin{array}{l}\text { Width } \\
\text { [min] }\end{array}$ & $\begin{array}{c}\text { Area } \\
{[\mathrm{mAU} * \mathrm{~s}]}\end{array}$ & $\begin{array}{l}\text { Height } \\
\text { [mAU] }\end{array}$ & $\begin{array}{c}\text { Area } \\
\%\end{array}$ \\
\hline$-\cdots|-\ldots-n|-\cdots \mid$ & $-\ldots-n$ & $|-\ldots+n|$ & $-\ldots-n \mid$ & $-\ldots-1$ \\
\hline $122.839 \mathrm{MM}$ & 1.6915 & $2.91476 \mathrm{e} 4$ & 287.19257 & 93.1726 \\
\hline 227.485 MM & 1.8349 & 2135.83789 & 19.40017 & 6.8274 \\
\hline Totals : & & $3.12834 \mathrm{e} 4$ & 306.59274 & \\
\hline
\end{tabular}




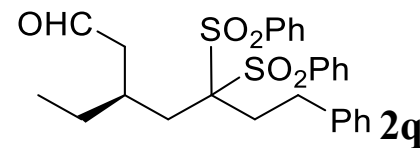

Data File D: \CHEM32\...05-AS-3-95-5-1ML-110MIN 2019-11-05 21-33-46\002-P2-D1-RAC-L-ETHYL.D Sample Name: RAC-L-ETHYL

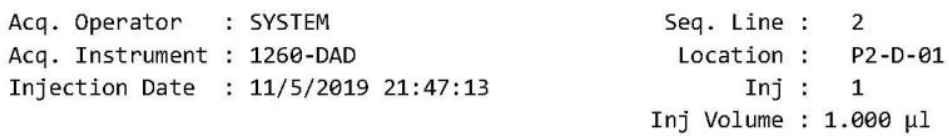

Different Inj Volume from Sample Entry! Actual Inj Volume : $3.000 \mu 1$

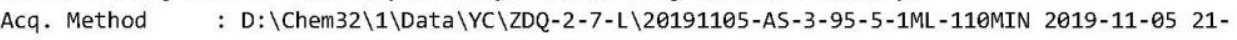
33-46\ZDQ-AS3-95-5-1ML-110MIN.M

Last changed : 11/5/2019 21:31:39 by SYSTEM

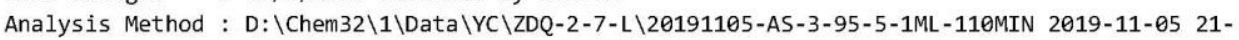
33-46\ZDQ-AS3-95-5-1ML-110MIN.M (Sequence Method)

Last changed : 11/13/2019 $19: 21: 16$ by SYSTEM (modified after loading)

Additional Info : Peak(s) manually integrated

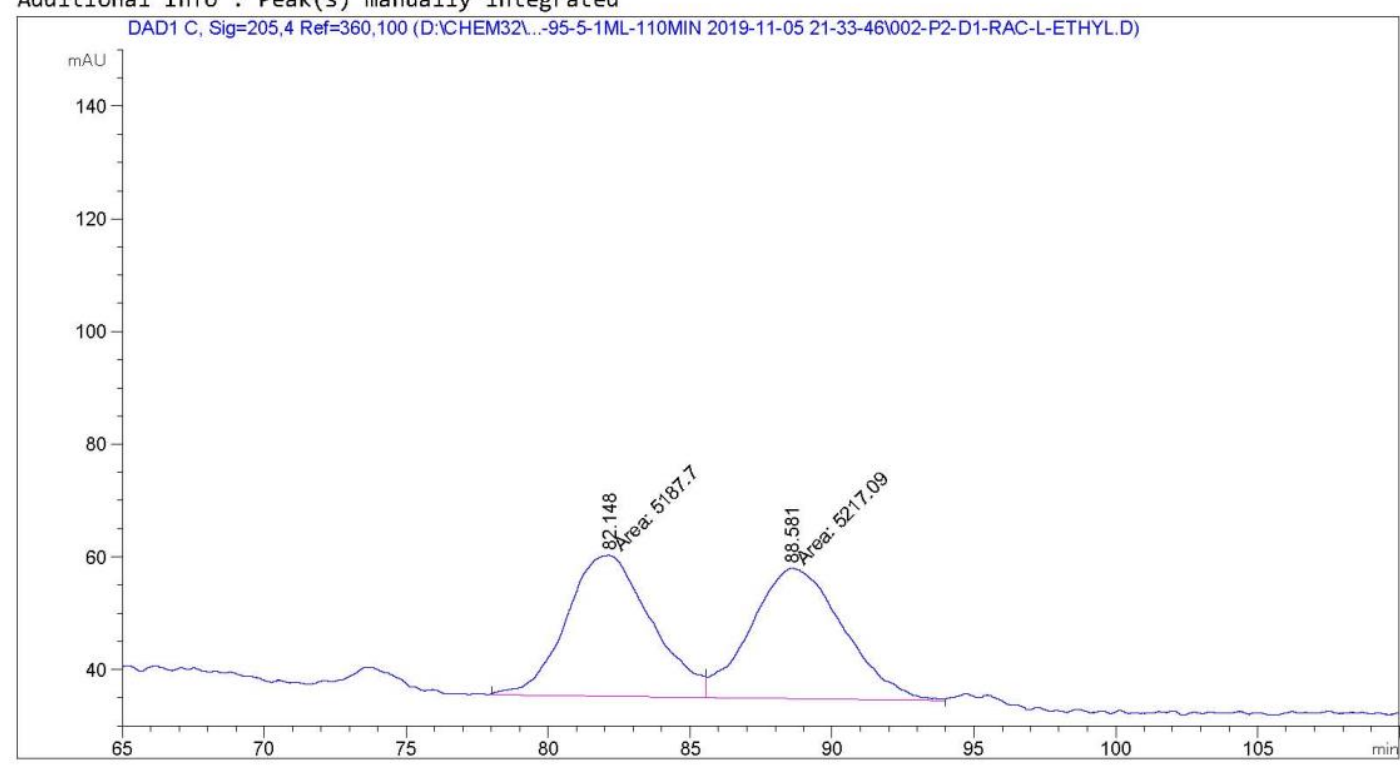

Area Percent Report

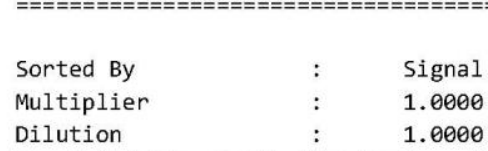

Use Multiplier \& Dilution Factor with ISTDs

Signal 1: DAD1 C, Sig=205,4 Ref $=360,100$

\begin{tabular}{|c|c|c|c|c|c|}
\hline $\begin{array}{c}\text { Peak } \\
\#\end{array}$ & $\begin{array}{l}\text { RetTime Type } \\
\text { [min] }\end{array}$ & $\begin{array}{l}\text { Width } \\
\text { [min] }\end{array}$ & $\begin{array}{c}\text { Area } \\
{[\mathrm{mAU} * \mathrm{~s}]}\end{array}$ & $\begin{array}{l}\text { Height } \\
\text { [mAU] }\end{array}$ & $\begin{array}{c}\text { Area } \\
\%\end{array}$ \\
\hline - & |- - & & מ & - & | \\
\hline 1 & 82.148 MF & 3.4503 & 5187.69824 & 25.05916 & 49.8588 \\
\hline 2 & $88.581 \mathrm{FM}$ & 3.7645 & 5217.08691 & 23.09765 & 50.1412 \\
\hline Tota & 1s: & & $1.04048 \mathrm{e} 4$ & 48.15681 & \\
\hline
\end{tabular}


Data File D: \Chem32\...191105-AS-3-95-5-1ML-110MIN 2019-11-05 21-33-46\003-P2-D2-L-ETHYL.D Sample Name: L-ETHYL

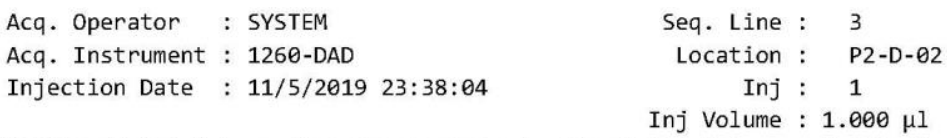

Different Inj Volume from Sample Entry! Actual Inj Volume : $3.000 \mu 1$

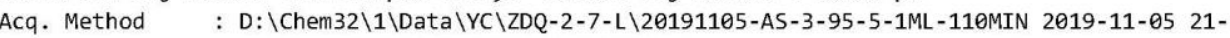
33-46\ZDQ-AS3-95-5-1ML-110MIN.M

Last changed : 11/5/2019 21:31:39 by SYSTEM

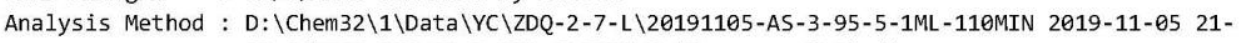
33-46\ZDQ-AS3-95-5-1ML-110MIN.M (Sequence Method)

Last changed : 11/13/2019 19:22:28 by SYSTEM (modified after loading)

Additional Info : Peak(s) manually integrated

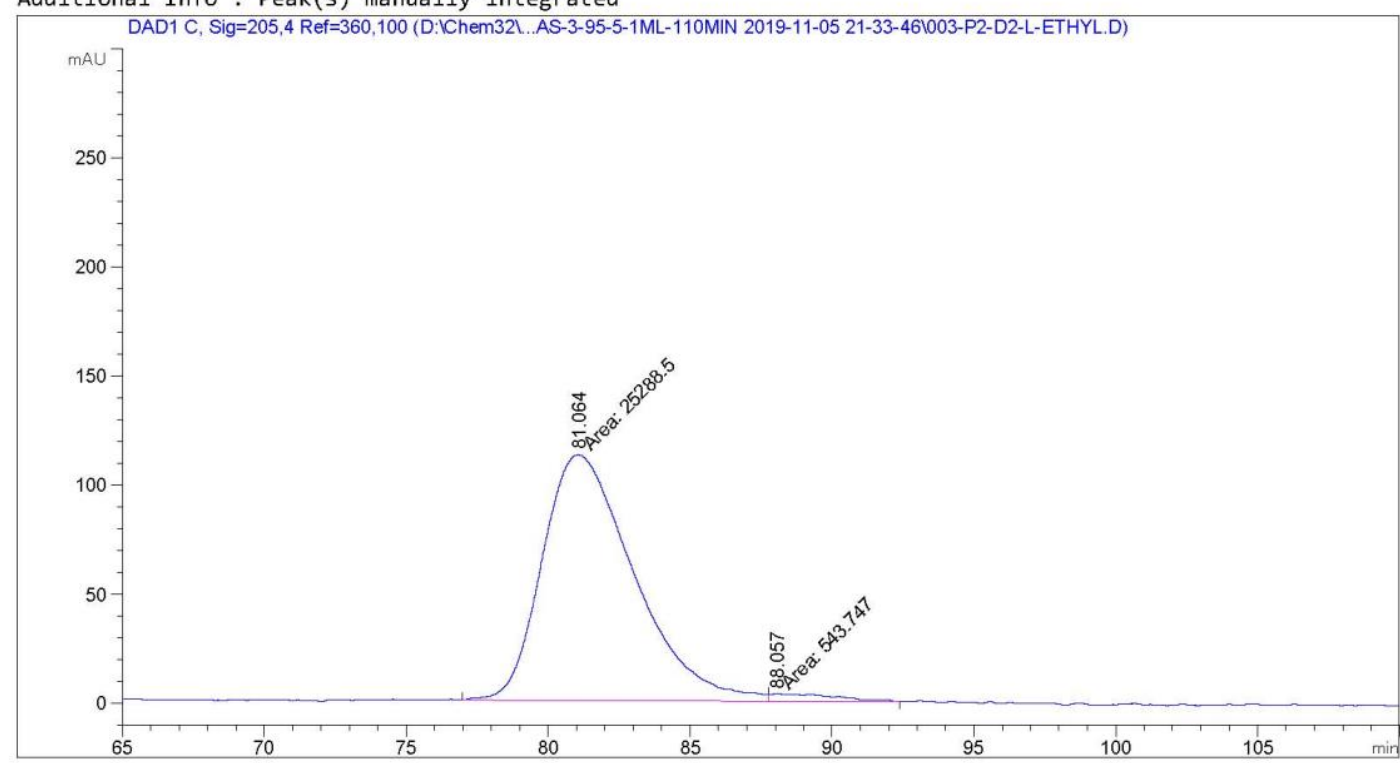

Area Percent Report

\begin{tabular}{|c|c|c|c|c|}
\hline Sorted By & : & Signal & & \\
\hline Multiplier & : & 1.0000 & & \\
\hline Dilution & : & 1.0000 & & \\
\hline \multicolumn{5}{|c|}{ Use Multiplier \& Dilution Factor with ISTDs } \\
\hline \multicolumn{5}{|c|}{ Signal 1: DAD1 C, Sig=205,4 Ref $=360,100$} \\
\hline $\begin{array}{l}\text { Peak RetTime Type } \\
\# \quad[\mathrm{~min}]\end{array}$ & $\begin{array}{l}\text { Width } \\
\text { [min] }\end{array}$ & $\begin{array}{c}\text { Area } \\
{[\mathrm{mAU} * \mathrm{~s}]}\end{array}$ & $\begin{array}{l}\text { Height } \\
\text { [mAU] }\end{array}$ & $\begin{array}{c}\text { Area } \\
\%\end{array}$ \\
\hline$-\cdots|-\ldots-n|-\cdots \mid$ & $-\ldots-n$ & $|-\ldots+n|$ & $-\ldots-n \mid$ & $-\ldots-n \mid$ \\
\hline $181.064 \mathrm{MF}$ & 3.7411 & $2.52885 \mathrm{e} 4$ & 112.66174 & 97.8951 \\
\hline $288.057 \mathrm{FM}$ & 2.6184 & 543.74677 & 3.46111 & 2.1049 \\
\hline Totals : & & $2.58323 e 4$ & 116.12286 & \\
\hline
\end{tabular}




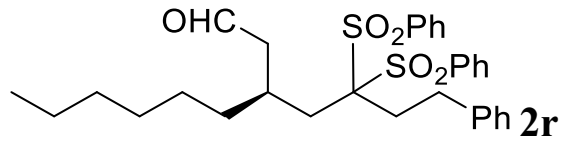

Data File D: \Chem32\...31-OD-H-90-10-1ML-35MIN 2019-10-31 22-13-29\002-P2-E8-RAC-L-ethyl.D Sample Name: RAC-L-ethyl

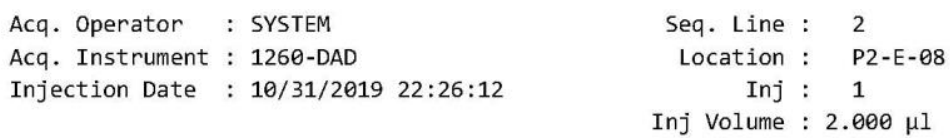

Different Inj Volume from Sample Entry! Actual Inj Volume : $3.000 \mu 1$

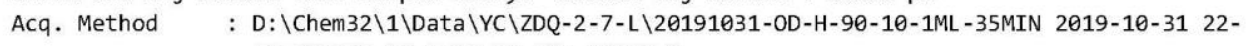

13-29\ZDQ-OD-H-90-10-1ML-35MIN.M

Last changed : 10/31/2019 23:00:38 by SYSTEM

(modified after loading)

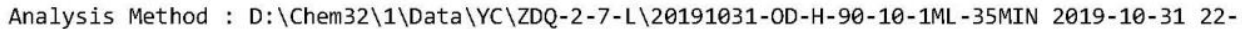
13-29\ZDQ-OD-H-90-10-1ML-35MIN.M (Sequence Method)

Last changed : 11/13/2019 20:41:32 by SYSTEM

(modified after loading)

Additional Info: Peak(s) manually integrated

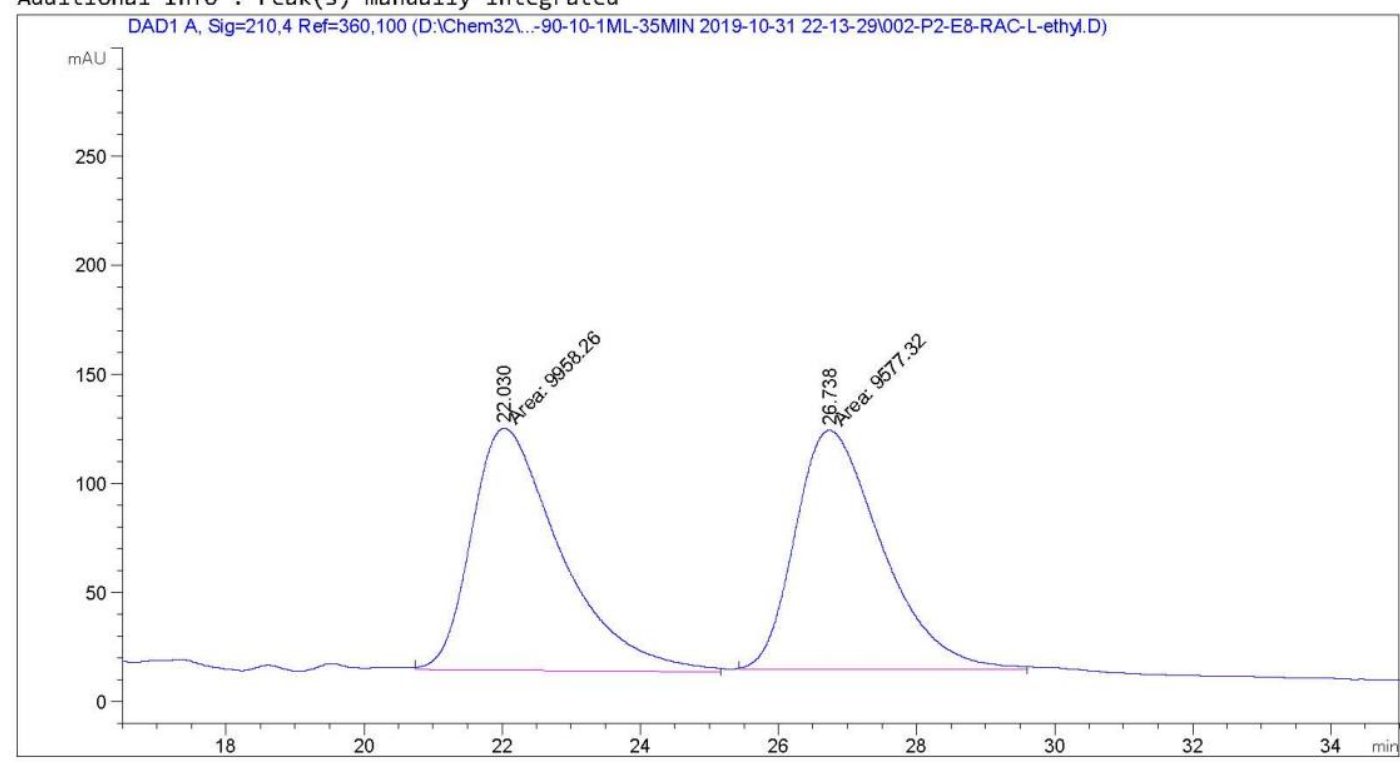

Area Percent Report

\begin{tabular}{|c|c|c|c|c|}
\hline Sorted By & $:$ & Signal & & \\
\hline Multiplier & : & 1.0000 & & \\
\hline Dilution & : & 1.0000 & & \\
\hline \multicolumn{5}{|c|}{ Use Multiplier \& Dilution Factor with ISTDs } \\
\hline \multicolumn{5}{|c|}{ Signal 1: DAD1 A, Sig=210,4 $\operatorname{Re} f=360,100$} \\
\hline $\begin{array}{l}\text { Peak RetTime Type } \\
\# \quad \text { [min] }\end{array}$ & $\begin{array}{l}\text { Width } \\
{[\mathrm{min}]}\end{array}$ & $\begin{array}{c}\text { Area } \\
{[\mathrm{mAU} * \mathrm{~s}]}\end{array}$ & $\begin{array}{l}\text { Height } \\
{[\mathrm{mAU}]}\end{array}$ & $\begin{array}{c}\text { Area } \\
\%\end{array}$ \\
\hline 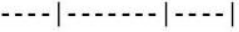 & - & |--ne-n & | & | \\
\hline $122.030 \mathrm{MM}$ & 1.4964 & 9958.25879 & 110.91486 & 50.9750 \\
\hline 226.738 MM & 1.4550 & 9577.32422 & 109.70274 & 49.0250 \\
\hline
\end{tabular}


Data File D: \Chem32\... 20191031-OD-H-90-10-1ML-35MIN 2019-10-31 22-13-29\003-P2-E9-Long.D Sample Name: Long

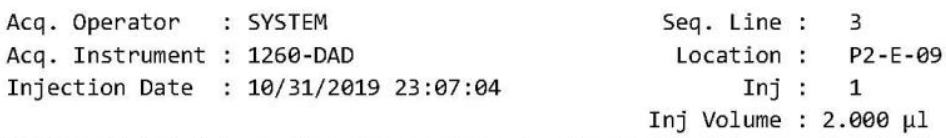

Different Inj Volume from Sample Entry! Actual Inj Volume : $3.000 \mu$

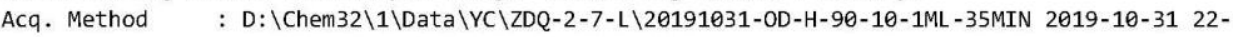
13-29\ZDQ-OD-H-90-10-1ML-35MIN.M

Last changed : 10/31/2019 23:00:38 by SYSTEM

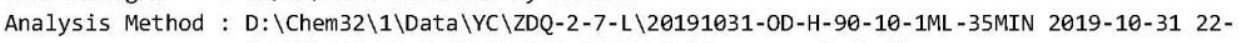
13-29\ZDQ-OD-H-90-10-1ML-35MIN.M (Sequence Method)

Last changed : 11/13/2019 20:42:34 by SYSTEM (modified after loading)

Additional Info : Peak(s) manually integrated

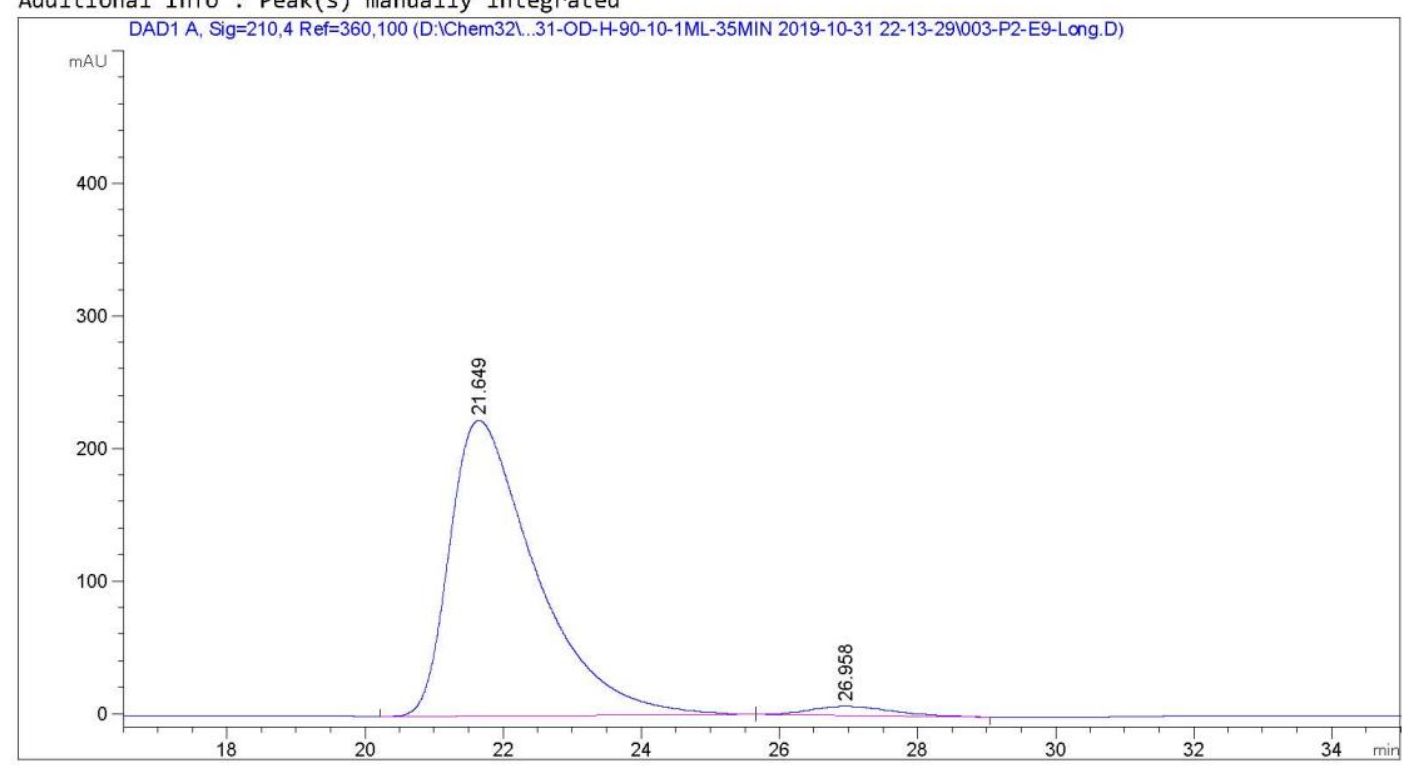

\section{Area Percent Report}

\begin{tabular}{|c|c|c|c|c|}
\hline Sorted By & $:$ & Signal & & \\
\hline Multiplier & : & 1.0000 & & \\
\hline Dilution & : & 1.0000 & & \\
\hline \multicolumn{5}{|c|}{ Use Multiplier \& Dilution Factor with ISTDs } \\
\hline \multicolumn{5}{|c|}{ Signal 1: DAD1 A, Sig $=210,4 \operatorname{Re} f=360,100$} \\
\hline Peak RetTime Type & Width & $\begin{array}{c}\text { Area } \\
{[\mathrm{mAU} * \mathrm{~s}]}\end{array}$ & Height & $\begin{array}{c}\text { Area } \\
\%\end{array}$ \\
\hline - & - & |-1-2]- & | & | \\
\hline $121.649 \mathrm{BB}$ & 1.2678 & $1.93017 \mathrm{e} 4$ & 223.00998 & 97.1589 \\
\hline $226.958 \mathrm{BB}$ & 0.9739 & 564.42297 & 6.87410 & 2.8411 \\
\hline Totals : & & $1.98661 \mathrm{e} 4$ & 229.88408 & \\
\hline
\end{tabular}


$\mathrm{OHC} \quad \mathrm{SO}_{2} \mathrm{Ph}$

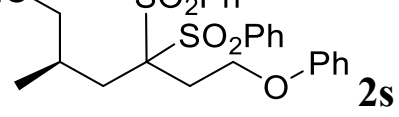

Data File D: \ChemSta...ata \YC\ZDQ-1-128\AS-85-15-1ML 2019-07-02 23-21-21 \relacatione0004.D Sample Name: ZDQ-1-129-2

\begin{tabular}{|c|c|c|c|}
\hline $\begin{array}{l}\text { Acq. Operator } \\
\text { Sample Operator }\end{array}$ & $\begin{array}{l}\text { : SYSTEM } \\
: \text { SYSTEM }\end{array}$ & Seq. Line & 4 \\
\hline Acq. Instrument & $: \mathrm{LC}$ & Location & P2-A-07 \\
\hline Injection Date & : 7/3/2019 1:40:43 AM & Inj & 1 \\
\hline
\end{tabular}

Acq. Method : D: \ChemStation $\backslash 1 \backslash$ Data $\backslash Y C \backslash Z D Q-1-128 \backslash A S-85-15-1 M L$ 2019-07-02 23-21-21 4 4-AS385-15-1ml-60min.M

Last changed : 4/9/2019 6:45:08 PM by SYSTEM

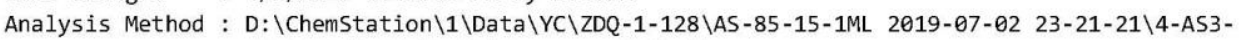
85-15-1ml-60min.M (Sequence Method)

Last changed : 11/13/2019 7:31:32 PM by SYSTEM (modified after loading)

Additional Info: Peak(s) manually integrated

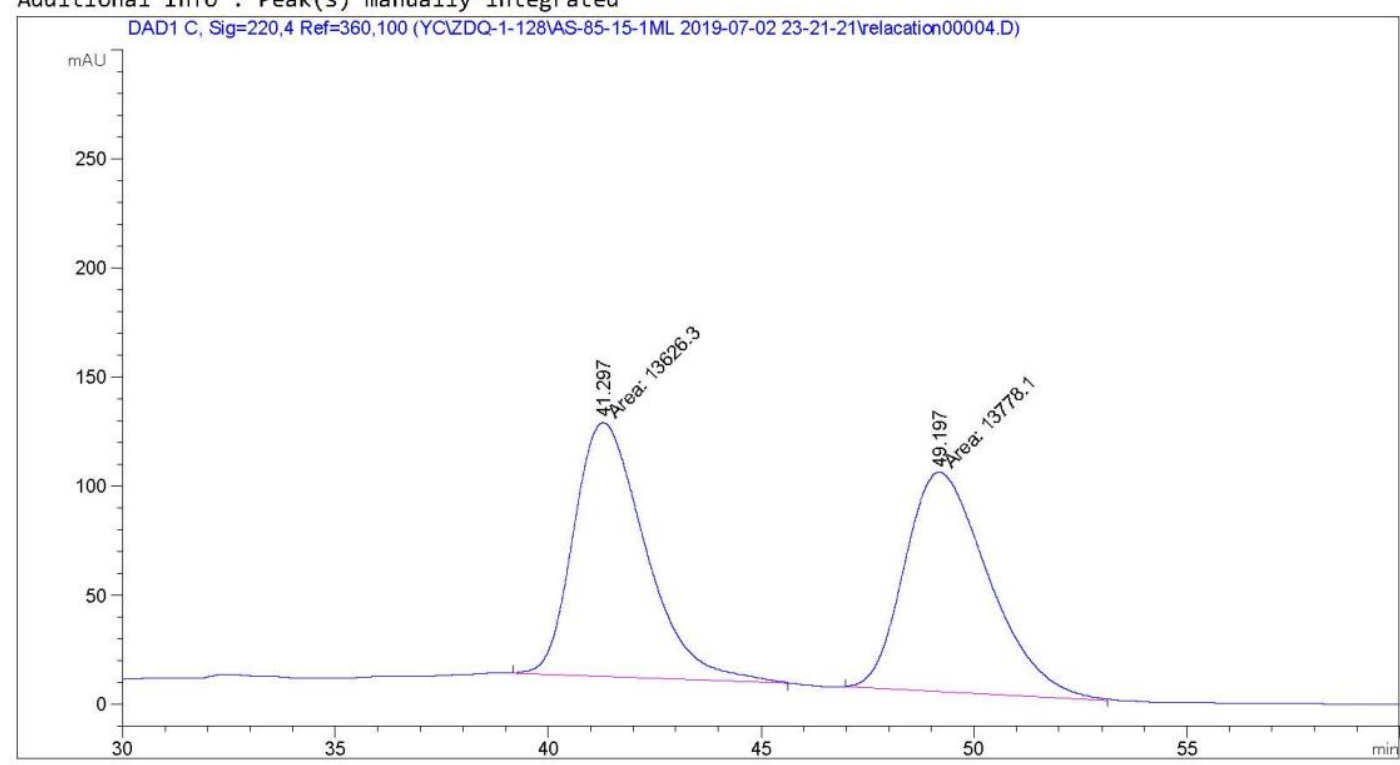

Area Percent Report

\begin{tabular}{|c|c|c|c|c|}
\hline Sorted By & : & Signal & & \\
\hline Multiplier & : & 1.0000 & & \\
\hline Dilution & : & 1.0000 & & \\
\hline \multicolumn{5}{|c|}{ Use Multiplier \& Dilution Factor with ISTDs } \\
\hline \multicolumn{5}{|c|}{ Signal 1: DAD1 C, Sig $=220,4 \operatorname{Re} f=360,100$} \\
\hline $\begin{array}{l}\text { Peak RetTime Type } \\
\# \quad \text { [min] }\end{array}$ & $\begin{array}{l}\text { Width } \\
\text { [min] }\end{array}$ & $\begin{array}{c}\text { Area } \\
{[\mathrm{mAU} * \mathrm{~s}]}\end{array}$ & $\begin{array}{l}\text { Height } \\
{[\mathrm{mAU}]}\end{array}$ & $\begin{array}{c}\text { Area } \\
\%\end{array}$ \\
\hline$-\ldots|--n|---\mid$ & $-\ldots-1$ & |-....... & $-2-2-1$ & $-\ldots-n$ \\
\hline $141.297 \mathrm{MM}$ & 1.9493 & $1.36263 \mathrm{e} 4$ & 116.50709 & 49.7231 \\
\hline 249.197 MM & 2.2828 & $1.37781 \mathrm{e} 4$ & 100.59157 & 50.2769 \\
\hline Totals : & & $2.74044 \mathrm{e} 4$ & 217.09866 & \\
\hline
\end{tabular}


Data File D: \ChemSta... ata \YC\ZDQ-1-128\AS-85-15-1ML 2019-07-02 23-21-21\relacatione0005.D Sample Name: ZDQ-1-128-2
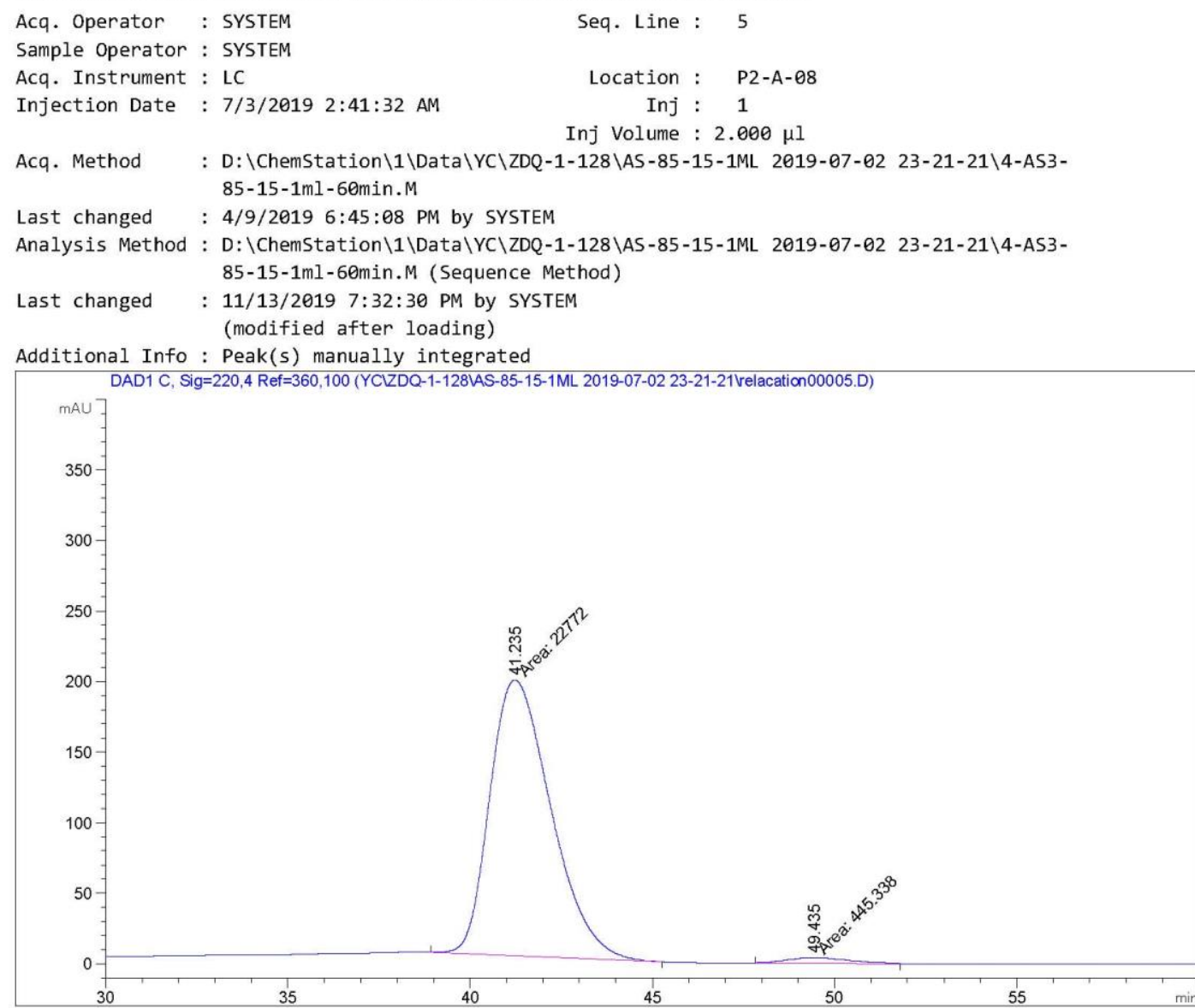

Area Percent Report

\begin{tabular}{|c|c|c|c|c|}
\hline Sorted By & : & Signal & & \\
\hline Multiplier & : & 1.0000 & & \\
\hline Dilution & : & 1.0000 & & \\
\hline \multicolumn{5}{|c|}{ Use Multiplier \& Dilution Factor with ISTDs } \\
\hline \multicolumn{5}{|c|}{ Signal 1: DAD1 C, Sig=220,4 Ref $=360,100$} \\
\hline $\begin{array}{l}\text { Peak RetTime Type } \\
\#[\text { min] }\end{array}$ & $\begin{array}{l}\text { Width } \\
\text { [min] }\end{array}$ & $\begin{array}{c}\text { Area } \\
{[\mathrm{mAU} * \mathrm{~s}]}\end{array}$ & $\begin{array}{l}\text { Height } \\
\text { [mAU] }\end{array}$ & $\begin{array}{c}\text { Area } \\
\%\end{array}$ \\
\hline 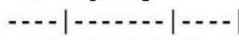 & $-\ldots-1$ & $|-\ldots+n|$ & 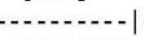 & 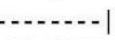 \\
\hline $141.235 \mathrm{MM}$ & 1.9407 & $2.27720 \mathrm{e} 4$ & 195.56349 & 98.0819 \\
\hline 249.435 MM & 1.9563 & 445.33789 & 3.79410 & 1.9181 \\
\hline Totals : & & $2.32173 \mathrm{e} 4$ & 199.35759 & \\
\hline
\end{tabular}

LC $11 / 13 / 2019$ 7:36:10 PM SYSTEM 
$\mathrm{OHC} \quad \mathrm{SO}_{2} \mathrm{Ph}$

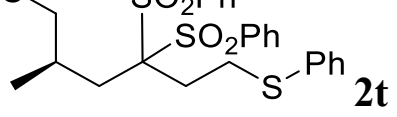

Data File D: \Chem32\...CHIRAL \S-AS-3-85-15-1ML-70MIN 2019-07-25 23-14-19\002-P2-A4-S-RAC.D Sample Name: S-RAC

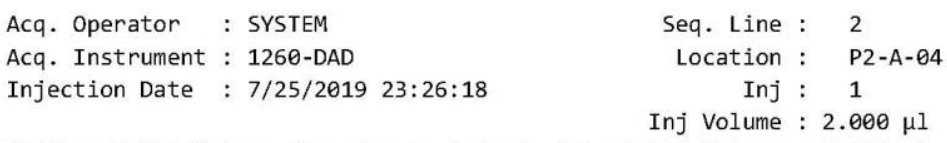

Different Inj Volume from Sample Entry! Actual Inj Volume : $4.000 \mu$

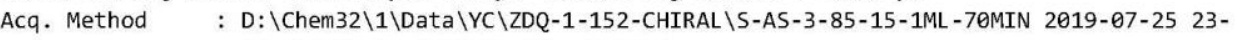
14-19\ZDQ-AS-3-85-15-1ML-7OMIN.M

Last changed : 7/25/2019 23:11:22 by SYSTEM

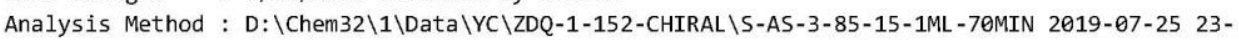
14-19\ZDQ-AS-3-85-15-1ML-70MIN.M (Sequence Method)

Last changed : 11/12/2019 $21: 11: 49$ by SYSTEM (modified after loading)

Additional Info : Peak(s) manually integrated

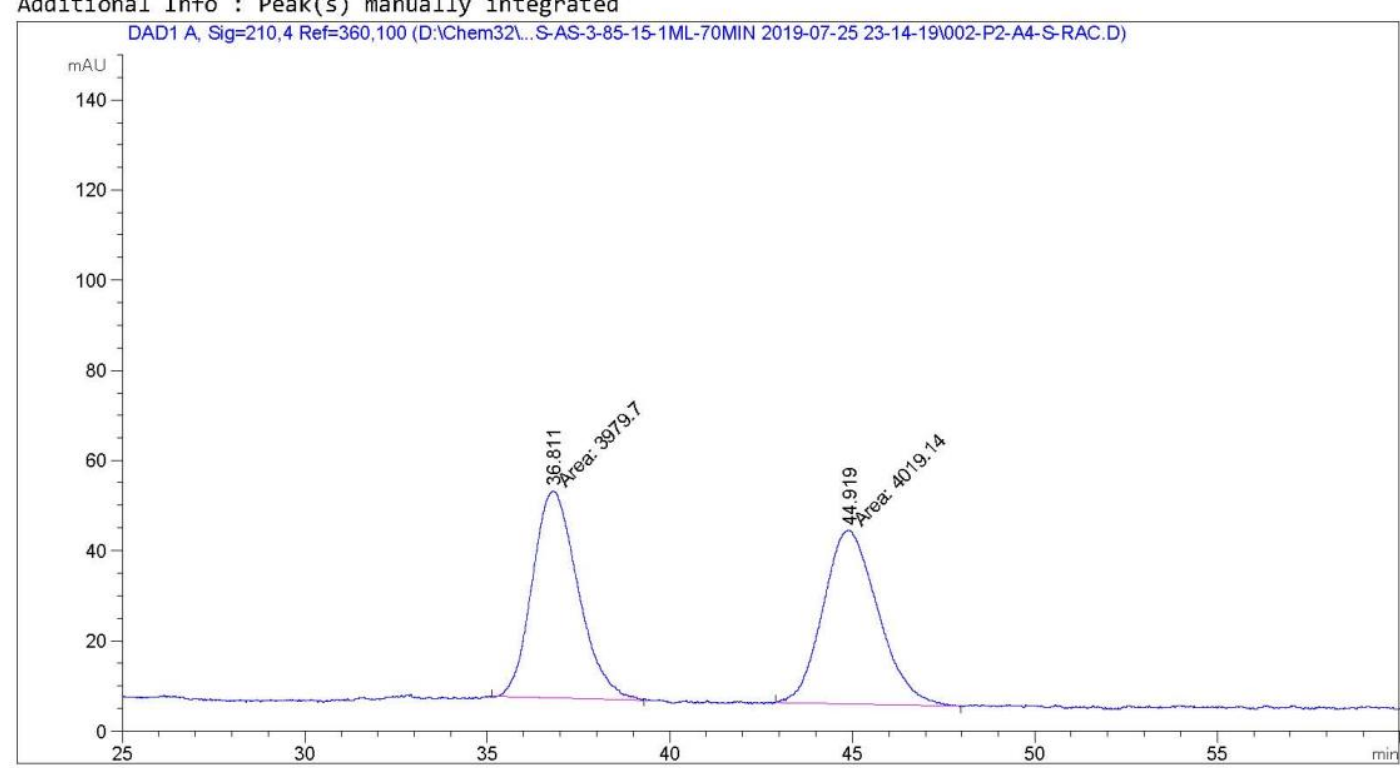

Area Percent Report

\begin{tabular}{|c|c|c|c|c|}
\hline Sorted By & : & Signal & & \\
\hline Multiplier & : & 1.0000 & & \\
\hline Dilution & : & 1.0000 & & \\
\hline \multicolumn{5}{|c|}{ Use Multiplier \& Dilution Factor with ISTDs } \\
\hline \multicolumn{5}{|c|}{ Signal 1: DAD1 A, Sig=210,4 $\operatorname{Re} f=360,100$} \\
\hline $\begin{array}{l}\text { Peak RetTime Type } \\
\# \quad \text { [min] }\end{array}$ & $\begin{array}{l}\text { Width } \\
\text { [min] }\end{array}$ & $\begin{array}{c}\text { Area } \\
{[\mathrm{mAU} * \mathrm{~s}]}\end{array}$ & $\begin{array}{l}\text { Height } \\
{[\mathrm{mAU}]}\end{array}$ & $\begin{array}{c}\text { Area } \\
\%\end{array}$ \\
\hline$-\ldots|--n|---\mid$ & $-2 .-1$ & $|-\ldots . .-2|$ & | & $-\ldots-n$ \\
\hline $136.811 \mathrm{MM}$ & 1.4463 & 3979.69727 & 45.85977 & 49.7534 \\
\hline 244.919 MM & 1.7375 & 4019.14185 & 38.55259 & 50.2466 \\
\hline Totals : & & 7998.83911 & 84.41235 & \\
\hline
\end{tabular}


Data File D: \Chem32\...152-CHIRAL \S-AS-3-85-15-1ML-70MIN 2019-07-25 23-14-19\003-P2-A5-S.D Sample Name: S

\begin{tabular}{|c|c|c|c|}
\hline Acq. Operator & : SYSTEM & Seq. Line : & 3 \\
\hline Acq. Instrument & : 1260-DAD & Location : & $P 2-A-05$ \\
\hline Injection Date & : 7/26/2019 00:37:08 & $\begin{array}{r}\text { Inj : } \\
\text { Inj Volume : }\end{array}$ & $\begin{array}{c}1 \\
: 2.000 \mu \mathrm{I}\end{array}$ \\
\hline
\end{tabular}

Acq. Method : D: \Chem32\1\Data \YC\ZDQ-1-152-CHIRAL \S-AS-3-85-15-1ML-70MIN 2019-07-25 2314-19\ZDQ-AS-3-85-15-1ML-70MIN. M

Last changed : 7/25/2019 23:11:22 by SYSTEM

Analysis Method : D: \Chem32\1\Data \YC\ZDQ-1-152-CHIRAL \S-AS-3-85-15-1ML-70MIN 2019-07-25 23-

14-19\ZDQ-AS-3-85-15-1ML-70MIN.M (Sequence Method)

Last changed : 11/12/2019 21:12:57 by SYSTEM

(modified after loading)

Additional Info: Peak(s) manually integrated

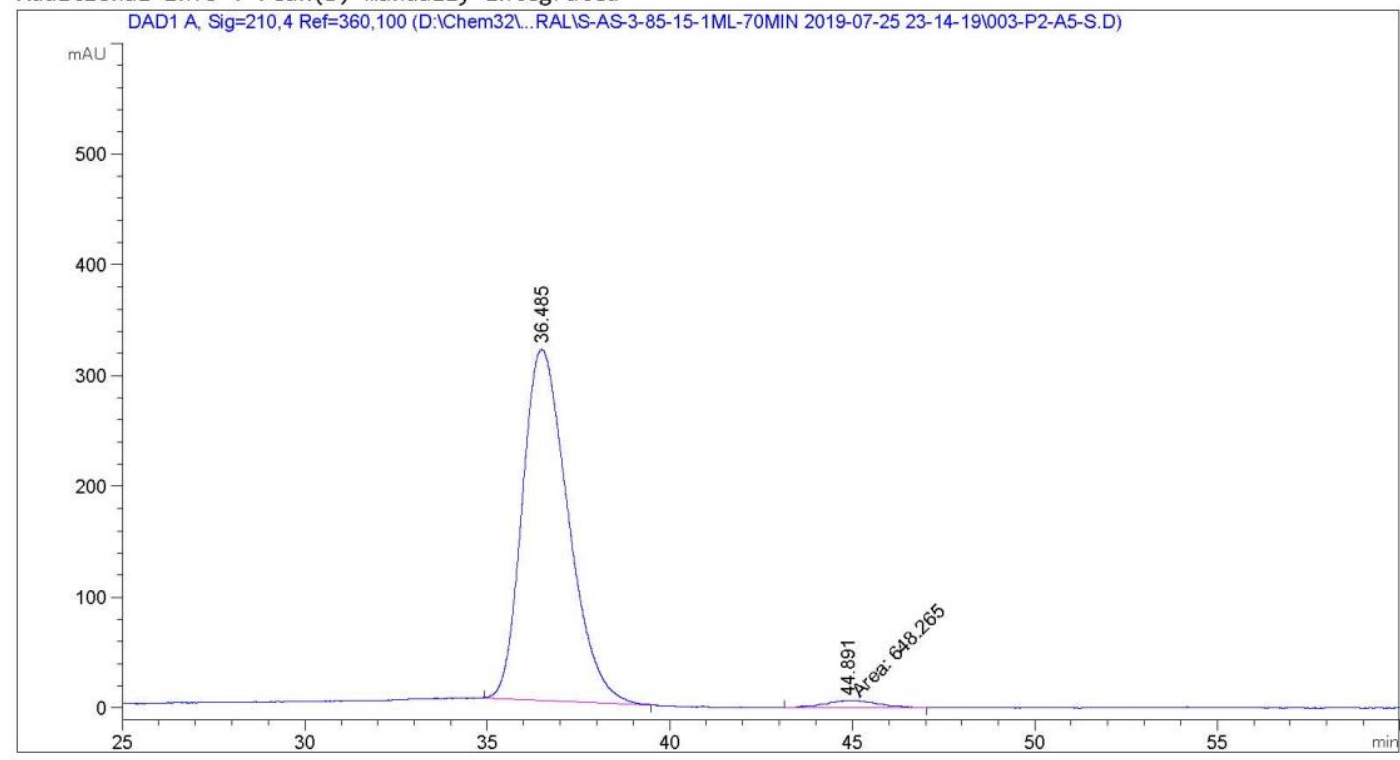

Area Percent Report

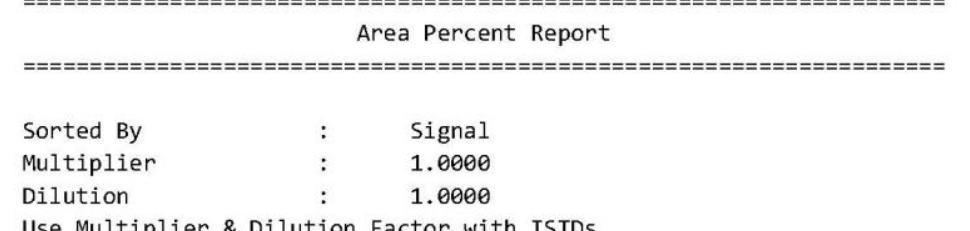

Use Multiplier \& Dilution Factor with ISTDs

Signal 1: DAD1 A, Sig=210,4 Ref $=360,100$

\begin{tabular}{|c|c|c|c|c|c|c|}
\hline $\begin{array}{c}\text { Peak } \\
\#\end{array}$ & $\begin{array}{c}\text { RetTime } \\
\text { [min] }\end{array}$ & Type & $\begin{array}{l}\text { Width } \\
\text { [min] }\end{array}$ & $\begin{array}{c}\text { Area } \\
{\left[\mathrm{mAU}^{*} \mathrm{~s}\right]}\end{array}$ & $\begin{array}{l}\text { Height } \\
\text { [mAU] }\end{array}$ & $\begin{array}{c}\text { Area } \\
\%\end{array}$ \\
\hline & & & & & . & \\
\hline 1 & 36. & & & $2.75102 \mathrm{e} 4$ & 316.70694 & 97.6978 \\
\hline 2 & 44.89 & & 1.7208 & 648.26508 & 6.27868 & 2.3022 \\
\hline
\end{tabular}

Totals : $\quad 2.81584 \mathrm{e} 4 \quad 322.98561$ 

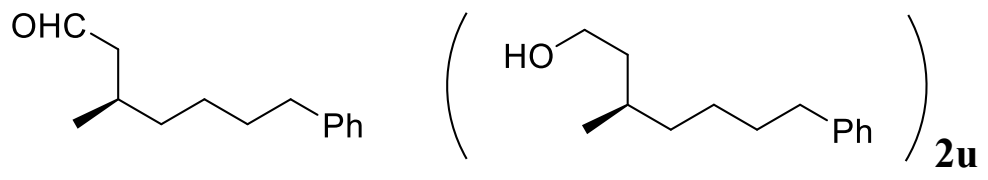

Data File D: \CHEM32\...0191128-AD-3-99.5-0.5-1ML-40MIN 2019-11-28 19-51-06\004-P2-C3-RAC.D Sample Name: RAC

\begin{tabular}{|c|c|c|c|}
\hline Acq. Operator & : SYSTEM & Seq. Line : & 4 \\
\hline Acq. Instrument & : 1260-DAD & Location : & P2-C-03 \\
\hline Injection Date & : 11/28/2019 21:34:51 & Inj : & 1 \\
\hline
\end{tabular}

Different Inj Volume from Sample Entry! Actual Inj Volume : $5.000 \mu \mathrm{l}$

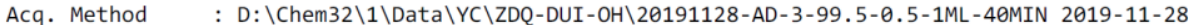

19-51-06\ZDQ-AD-3-99.5-0.5-1ML-4OMIN.M

Last changed : 11/28/2019 20:54:55 by SYSTEM

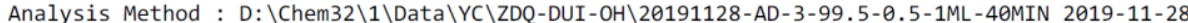
19-51-06\ZDQ-AD-3-99.5-0.5-1ML-40MIN.M (Sequence Method)

Last changed : 3/27/2020 20:27:02 by SYSTEM (modified after loading)

Additional Info : Peak(s) manually integrated

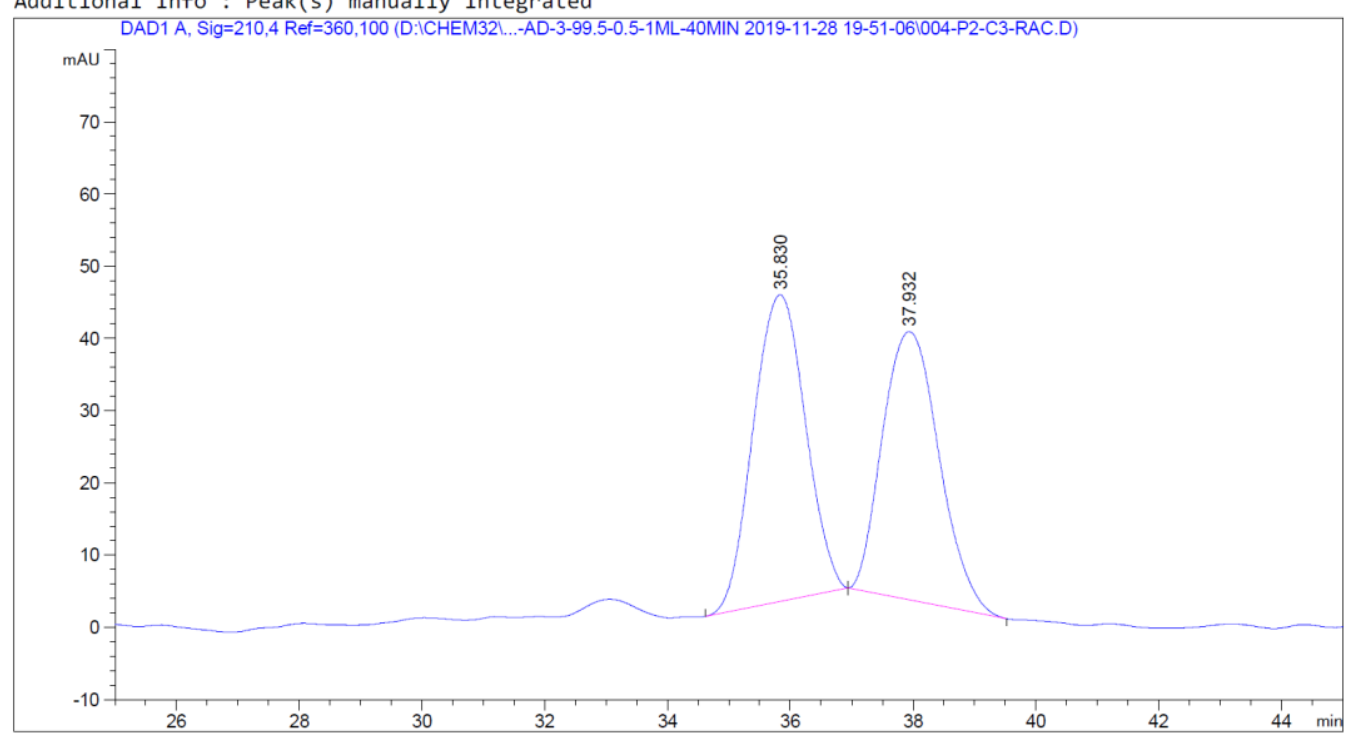

Area Percent Report

$\begin{array}{lll}=============================== \\ \text { Sorted By } & : & \text { Signal } \\ \text { Multiplier } & : & 1.0000 \\ \text { Dilution } & : & 1.0000\end{array}$

Use Multiplier \& Dilution Factor with ISTDs

Signal 1: DAD1 A, Sig=210,4 Ref=360,100

\begin{tabular}{|c|c|c|c|c|c|c|}
\hline $\begin{array}{c}\text { Peak } \\
\text { \# }\end{array}$ & $\begin{array}{c}\text { RetTime } \\
\text { [min] }\end{array}$ & Type & $\begin{array}{l}\text { Width } \\
\text { [min] }\end{array}$ & $\begin{array}{c}\text { Area } \\
{\left[\mathrm{mAU}^{*} \mathrm{~s}\right]}\end{array}$ & $\begin{array}{l}\text { Height } \\
{[\mathrm{mAU}]}\end{array}$ & $\begin{array}{c}\text { Area } \\
\%\end{array}$ \\
\hline--1 & ....... & & & $|-\ldots+n|$ & - & - \\
\hline 1 & 35.830 & BB & 0.9368 & 2506.78027 & 42.45936 & 51.2534 \\
\hline 2 & 37.932 & BB & 1.0069 & 2384.17114 & 37.14256 & 48.7466 \\
\hline & & & & 4890.95142 & 79.60192 & \\
\hline
\end{tabular}


Data File D: \Chem32\..\20191128-AD-3-99.5-0.5-1ML-40MIN 2019-11-28 19-51-06\003-P2-C4-C.D Sample Name: C

\begin{tabular}{|c|c|c|c|}
\hline Acq. Operator & : SYSTEM & Seq. Line : & 3 \\
\hline Acq. Instrument & $: 1260-D A D$ & Location : & $P 2-C-\theta$ \\
\hline Injection Date & : 11/28/2019 20:43:59 & $\begin{array}{r}\text { Inj : } \\
\text { Inj Volume : }\end{array}$ & $\begin{array}{l}1 \\
2.000 \mu l\end{array}$ \\
\hline
\end{tabular}

Different Inj Volume from Sample Entry! Actual Inj Volume : $5.000 \mu \mathrm{l}$

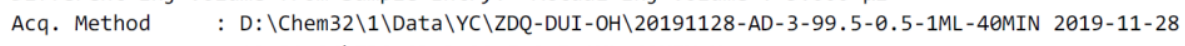
19-51-06\ZDQ-AD-3-99.5-0.5-1ML-40MIN.M

Last changed : 11/28/2019 20:54:55 by SYSTEM

(modified after loading)

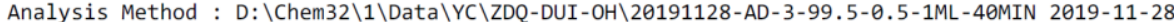
19-51-06\ZDQ-AD-3-99.5-0.5-1ML-40MIN.M (Sequence Method)

Last changed : 3/27/2020 20:27:31 by SYSTEM (modified after loading)

Additional Info: Peak(s) manually integrated

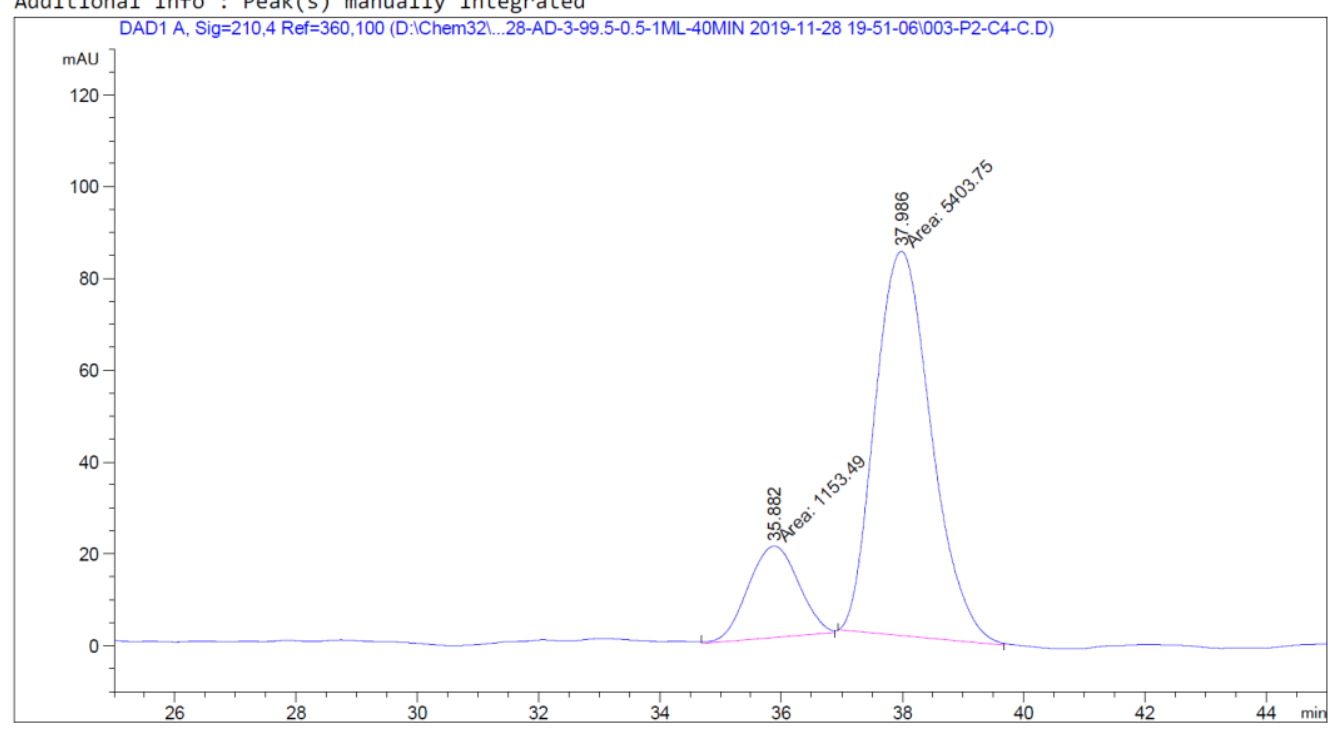

Area Percent Report

\begin{tabular}{|c|c|c|c|c|}
\hline Sorted By & : & Signal & & \\
\hline Multiplier & : & 1.0000 & & \\
\hline Dilution & : & 1.0000 & & \\
\hline \multicolumn{5}{|c|}{ Use Multiplier \& Dilution Factor with ISTDs } \\
\hline \multicolumn{5}{|c|}{ Signal 1: DAD1 A, Sig=210,4 $\operatorname{Ref}=360,100$} \\
\hline $\begin{array}{l}\text { Peak RetTime Type } \\
\# \text { [min] }\end{array}$ & $\begin{array}{l}\text { Width } \\
\text { [min] }\end{array}$ & $\begin{array}{c}\text { Area } \\
{[\mathrm{mAU} * \mathrm{~s}]}\end{array}$ & $\begin{array}{l}\text { Height } \\
{[\mathrm{mAU}]}\end{array}$ & $\begin{array}{c}\text { Area } \\
\%\end{array}$ \\
\hline | & - & $|-\ldots-\ldots|$ & | & $-\ldots$ \\
\hline $135.882 \mathrm{MM}$ & 0.9636 & 1153.48608 & 19.95079 & 17.5911 \\
\hline $237.986 \mathrm{MM}$ & 1.0756 & 5403.74609 & 83.73435 & 82.4089 \\
\hline
\end{tabular}


Data File D: \CHEM32\...0191114-AS-H-99.5-0.5-1ML-80MIN 2019-11-14 18-24-42\ө02-P2-E6-RAC.D Sample Name: RAC
Acq. Operator : SYSTEM
Seq. Line :
Acq. Instrument : 1260-DAD
Location : P2-E-06
Injection Date : 11/14/2019 18:28:44
Inj : 1
Inj Volume : $2.000 \mu \mathrm{l}$

Different Inj Volume from Sample Entry! Actual Inj Volume : $3.000 \mu$

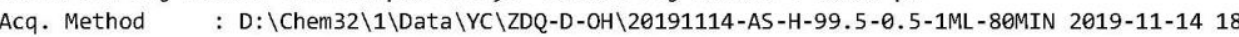

-24-42\ZDQ-AS-H-99.5-0.5-1ML-80MIN.M

Last changed : 11/14/2019 19:23:48 by SYSTEM

(modified after loading)

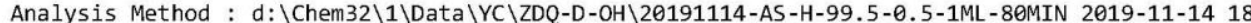

-24-42 \ZDQ-AS-H-99.5-0.5-1ML-80MIN.M (Sequence Method)

Last changed : 11/14/2019 20:37:20 by SYSTEM

(modified after loading)

Additional Info : Peak(s) manually integrated

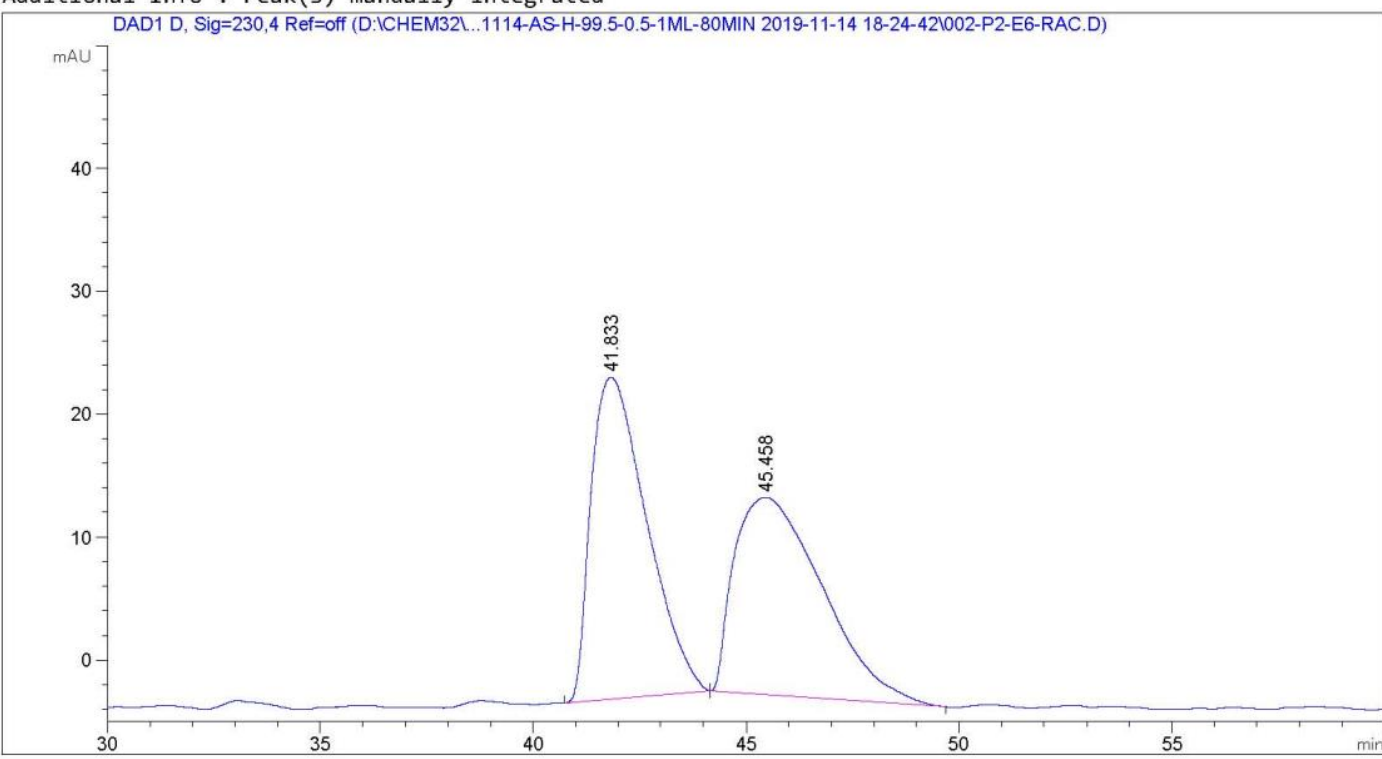

Area Percent Report

\begin{tabular}{|c|c|c|c|c|}
\hline Sorted By & : & Signal & & \\
\hline Multiplier & : & 1.0000 & & \\
\hline Dilution & : & 1.0000 & & \\
\hline \multicolumn{5}{|c|}{ Use Multiplier \& Dilution Factor with ISTDs } \\
\hline \multicolumn{5}{|c|}{ Signal 1: DAD1 D, Sig $=230,4$ Ref $=0 f f$} \\
\hline $\begin{array}{l}\text { Peak RetTime Type } \\
\# \quad[\text { min] }\end{array}$ & $\begin{array}{l}\text { Width } \\
{[\min ]}\end{array}$ & $\begin{array}{c}\text { Area } \\
{\left[\mathrm{mAU}^{*} \mathrm{~s}\right]}\end{array}$ & $\begin{array}{l}\text { Height } \\
\text { [mAU] }\end{array}$ & $\begin{array}{c}\text { Area } \\
\%\end{array}$ \\
\hline$-\ldots|-\ldots-1|-\ldots \mid$ & $-\ldots-\ldots$ & $-\ldots-1$ & | & $-\ldots-1$ \\
\hline $141.833 \mathrm{BB}$ & 1.3092 & 2373.16431 & 26.18857 & 50.6995 \\
\hline $245.458 \mathrm{BB}$ & 1.6880 & 2307.67676 & 16.04440 & 49.3005 \\
\hline
\end{tabular}


Data File d: \Chem32\...20191114-AS-H-99.5-0.5-1ML-80MIN 2019-11-14 18-24-42\003-P2-E7-OH.D Sample Name: $\mathrm{OH}$

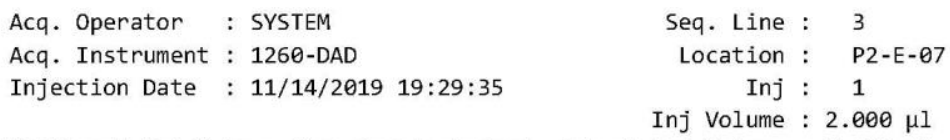

Different Inj Volume from Sample Entry! Actual Inj Volume : $3.000 \mu 1$

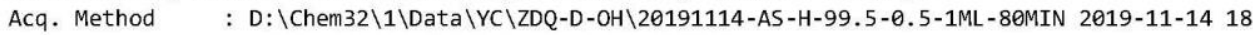
-24-42\ZDQ-AS-H-99.5-0.5-1ML-80MIN.M

Last changed : 11/14/2019 19:23:48 by SYSTEM

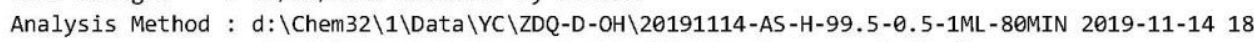
-24-42 \ZDQ-AS-H-99.5-0.5-1ML-80MIN.M (Sequence Method)

Last changed : 11/14/2019 20:38:37 by SYSTEM (modified after loading)

Additional Info : Peak(s) manually integrated

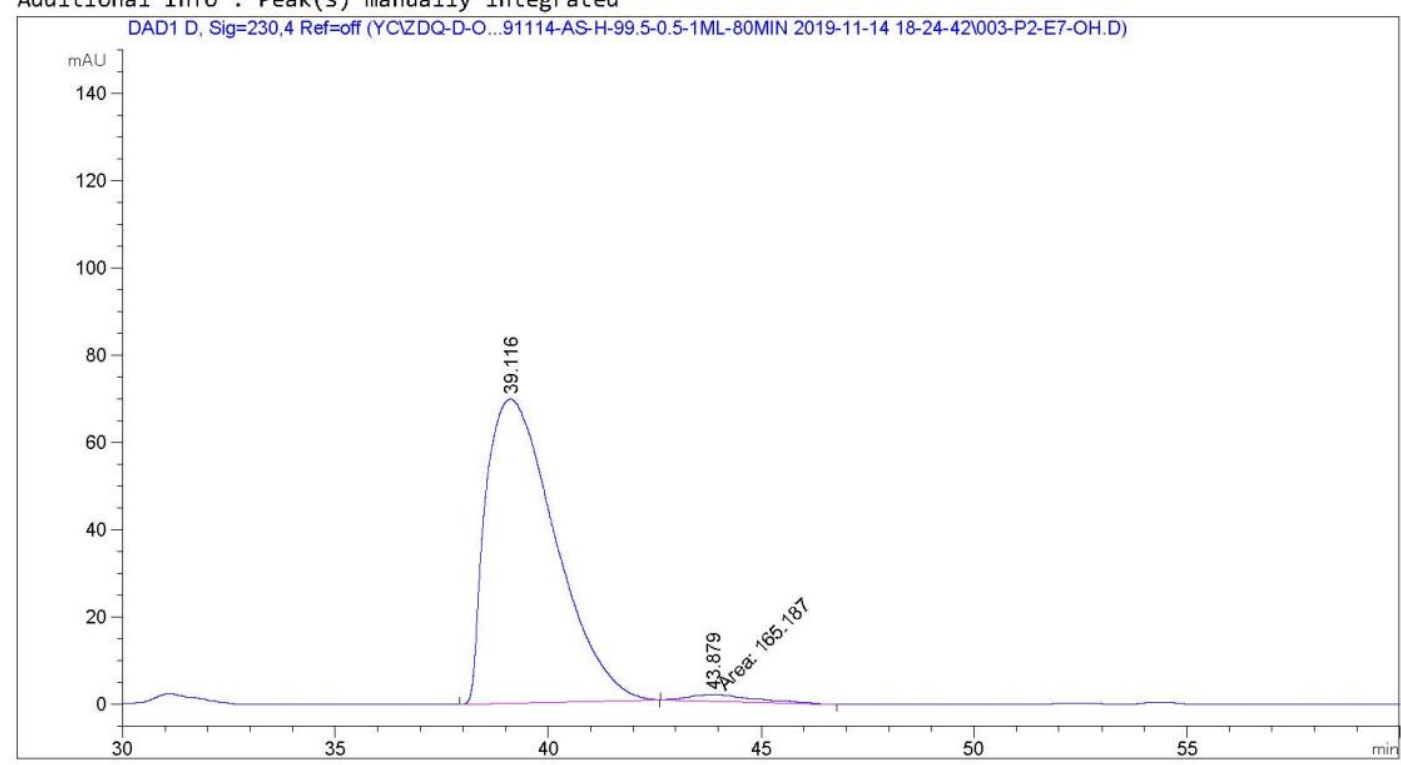

Area Percent Report

\begin{tabular}{|c|c|c|c|c|}
\hline Sorted By & : & Signal & & \\
\hline Multiplier & : & 1.0000 & & \\
\hline Dilution & : & 1.0000 & & \\
\hline \multicolumn{5}{|c|}{ Use Multiplier \& Dilution Factor with ISTDs } \\
\hline \multicolumn{5}{|c|}{ Signal 1: DAD1 D, Sig=230,4 Ref=off } \\
\hline $\begin{array}{l}\text { Peak RetTime Type } \\
\# \quad[\mathrm{~min}]\end{array}$ & $\begin{array}{l}\text { Width } \\
\text { [min] }\end{array}$ & $\begin{array}{c}\text { Area } \\
{[\mathrm{mAU} * \mathrm{~s}]}\end{array}$ & $\begin{array}{l}\text { Height } \\
\text { [mAU] }\end{array}$ & $\begin{array}{c}\text { Area } \\
\%\end{array}$ \\
\hline$-\cdots|-\ldots-n|-\cdots \mid$ & $-\ldots-n$ & | - - n- & $\mid$ & $-\ldots-n \mid$ \\
\hline $139.116 \mathrm{BB}$ & 1.6278 & 7877.85840 & 69.74339 & 97.9462 \\
\hline 243.879 MM & 1.7991 & 165.18703 & 1.53026 & 2.0538 \\
\hline Totals : & & 8043.04543 & 71.27365 & \\
\hline
\end{tabular}




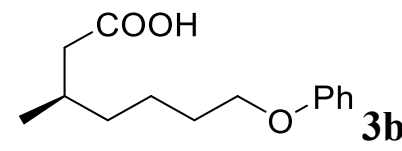

Data File D: \ChemSta...191029-AS-3-90-10-0.8ml-30mIN 2019-10-29 16-11-32\relacatione0002.D Sample Name: RAC

\begin{tabular}{|c|c|c|c|}
\hline Acq. Operator & : SYSTEM & Seq. Line : & : \\
\hline Sample Operator & : SYSTEM & & \\
\hline Acq. Instrument & : LC & Location : & $P 1-F-0$ \\
\hline Injection Date & : 10/29/2019 4:28:01 PM & $\begin{array}{r}\text { Inj : } \\
\text { Inj Volume : }\end{array}$ & $\begin{array}{lc}: & 1 \\
: & 2.000\end{array}$ \\
\hline
\end{tabular}

Different Inj Volume from Sample Entry! Actual Inj Volume : $4.000 \mu 1$

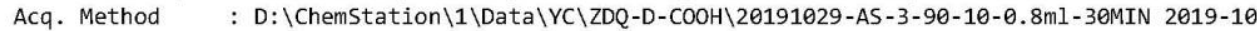
-29 16-11-32\4-AS3-90-10-0.8ml-30min.M

Last changed : 10/29/2019 4:09:14 PM by SYSTEM

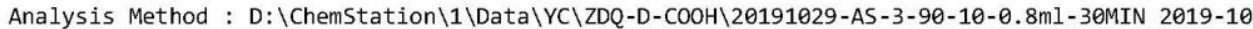

-29 16-11-32\4-AS3-90-10-0.8ml-30min.M (Sequence Method)

Last changed : 11/13/2019 8:47:19 PM by SYSTEM

(modified after loading)

Additional Info : Peak(s) manually integrated

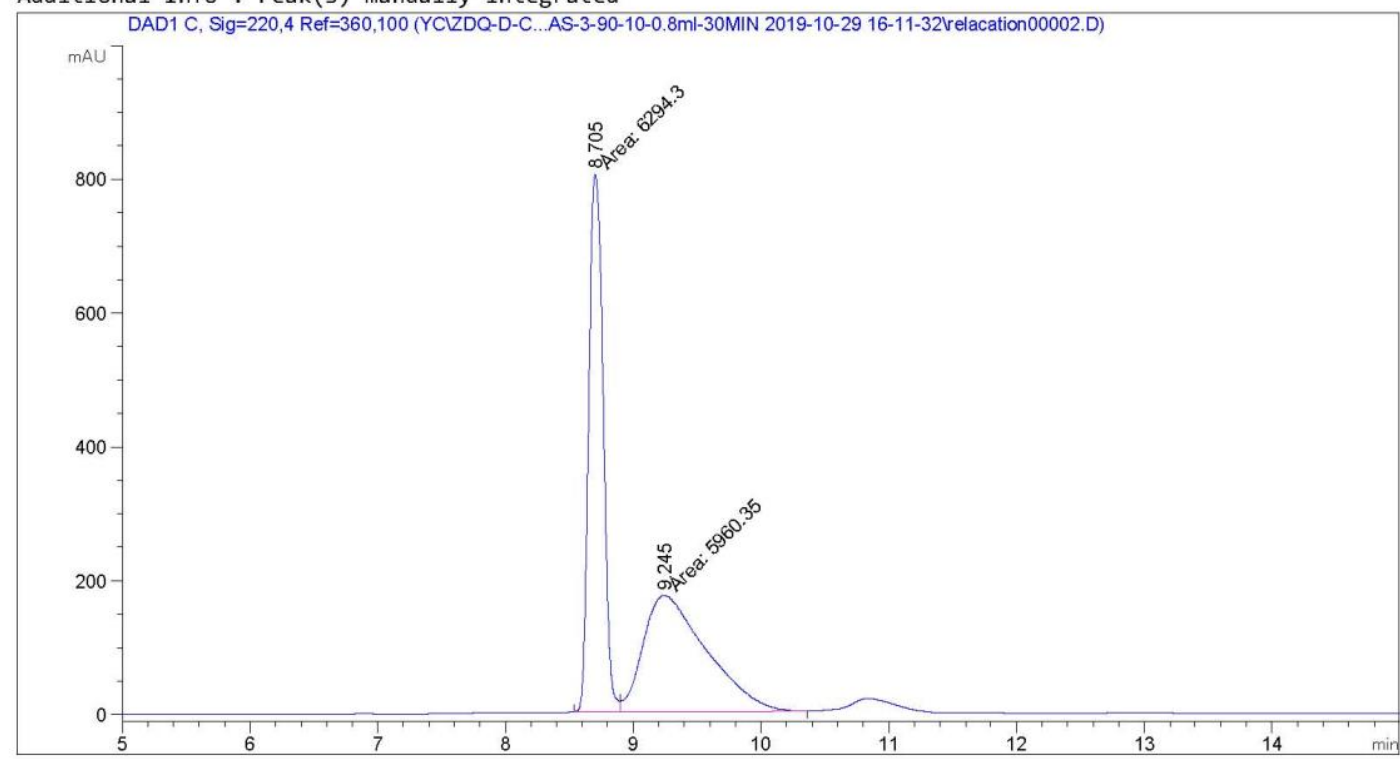

Area Percent Report

\begin{tabular}{|c|c|c|c|c|}
\hline Sorted By & : & Signal & & \\
\hline Multiplier & : & 1.0000 & & \\
\hline Dilution & : & 1.0000 & & \\
\hline \multicolumn{5}{|c|}{ Use Multiplier \& Dilution Factor with ISTDs } \\
\hline \multicolumn{5}{|c|}{ Signal 1: DAD1 C, Sig=220,4 $\operatorname{Ref}=360,100$} \\
\hline $\begin{array}{l}\text { Peak RetTime Type } \\
\# \quad \text { [min] }\end{array}$ & $\begin{array}{l}\text { Width } \\
\text { [min] }\end{array}$ & $\begin{array}{c}\text { Area } \\
{[\mathrm{mAU} * \mathrm{~s}]}\end{array}$ & $\begin{array}{l}\text { Height } \\
{[\mathrm{mAU}]}\end{array}$ & $\begin{array}{c}\text { Area } \\
\%\end{array}$ \\
\hline$-\cdots|-\cdots--\infty|-\cdots \mid$ & $-\cdots-\cdots$ & $-\cdots-1$ & | & | \\
\hline $18.705 \mathrm{MF}$ & 0.1305 & 6294.30371 & 804.14063 & 51.3626 \\
\hline $9.245 \mathrm{FM}$ & 0.5721 & 5960.35205 & 173.63161 & 48.6374 \\
\hline
\end{tabular}


Data File D: \ChemSta...191029-AS-3-90-10-0.8ml-30MIN 2019-10-29 16-11-32\relacatione0003.D Sample Name: $\mathrm{COOH}$

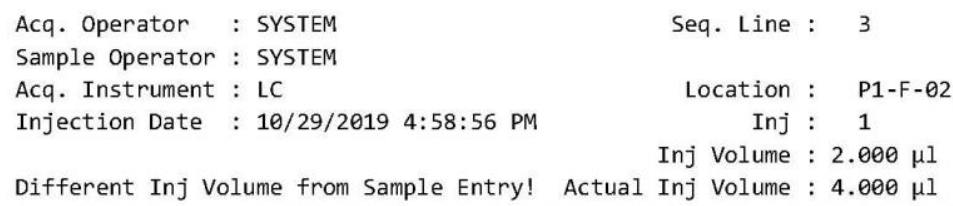

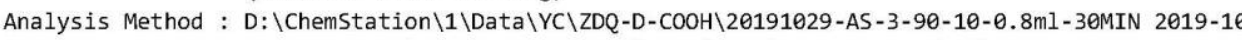
-29 16-11-32\4-AS3-90-10-0.8ml-30min.M (Sequence Method)

Last changed : 11/13/2019 8:47:19 PM by SYSTEM (modified after loading)

Additional Info : Peak(s) manually integrated

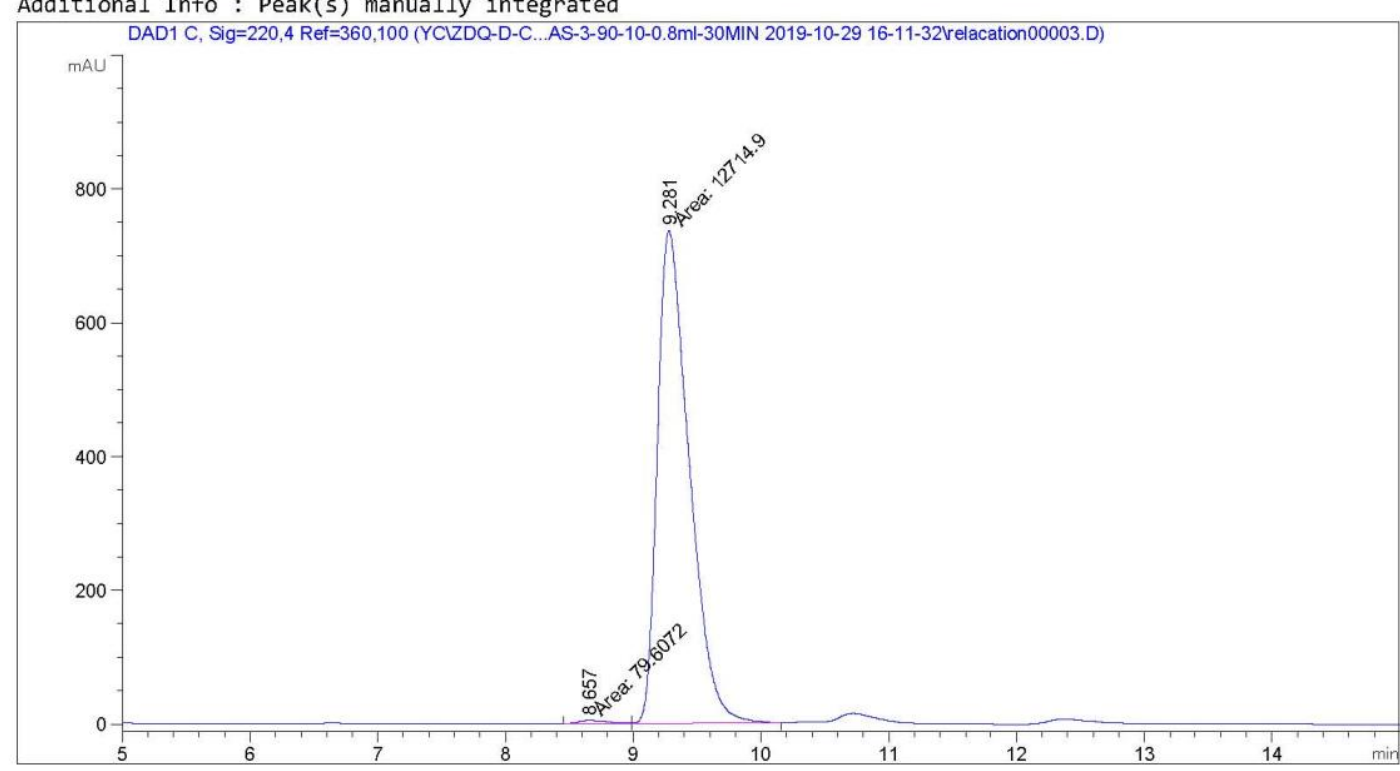

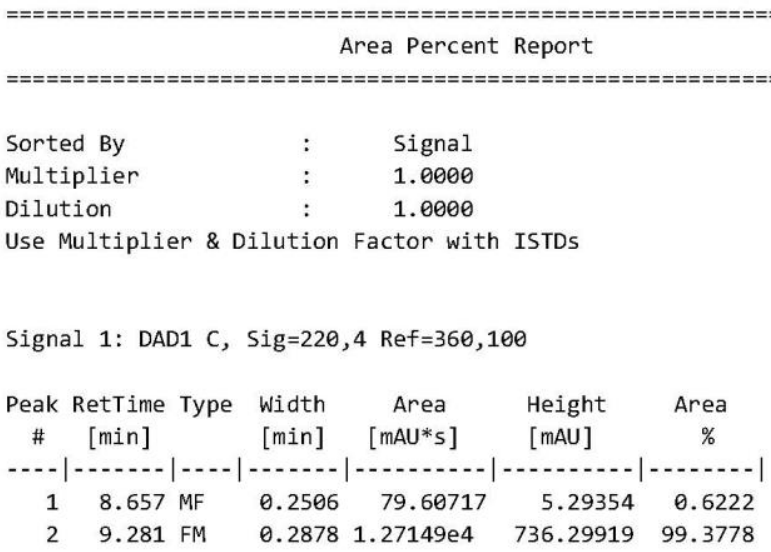




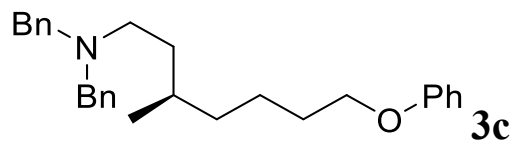

Data File D: \CHEM32\...N $20191108-O D-H-85-15-1 M L-30 M I N$ 2019-11-08 09-02-13\002-P2-D1-RAC.D Sample Name: RAC

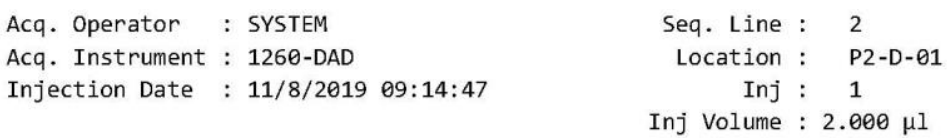

Different Inj Volume from Sample Entry! Actual Inj Volume : $3.000 \mu 1$

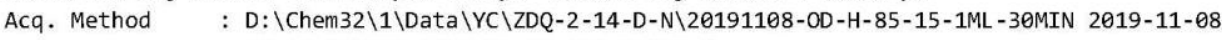
09-02-13\ZDQ-OD-H-85-15-1ML -30MIN.M

Last changed : 11/8/2019 09:01:02 by SYSTEM

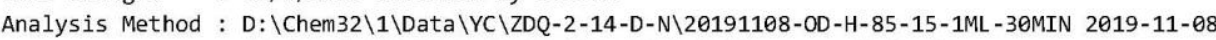
09-02-13\ZDQ-OD-H-85-15-1ML-30MIN.M (Sequence Method)

Last changed : 11/13/2019 20:45:43 by SYSTEM (modified after loading)

Additional Info: Peak(s) manually integrated

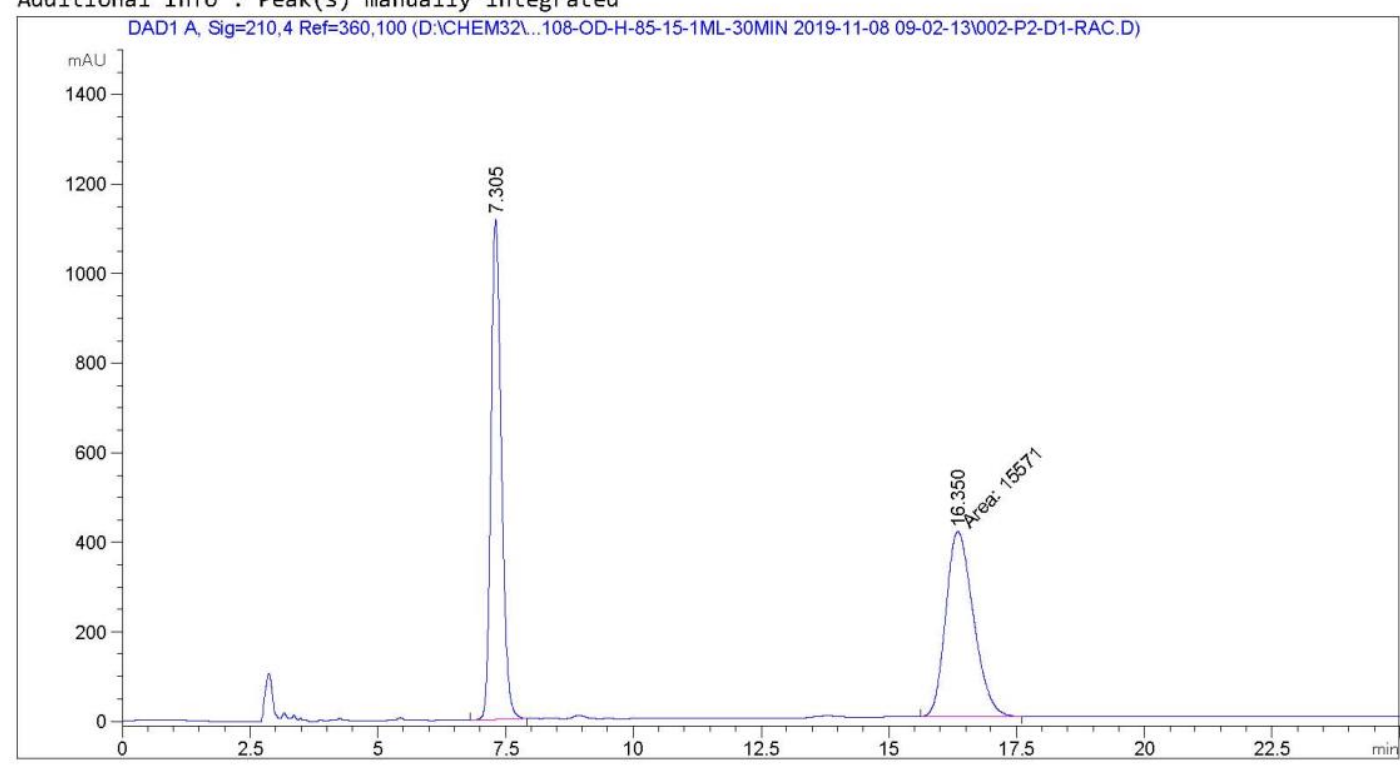

Area Percent Report

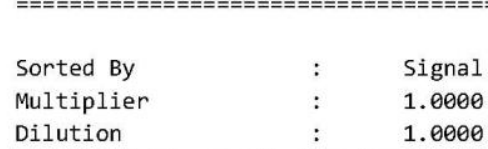

Use Multiplier \& Dilution Factor with ISTDs

Signal 1: DAD1 A, Sig=210,4 Ref $=360,100$

$$
\begin{aligned}
& \text { Peak RetTime Type Width Area Height Area } \\
& \begin{array}{cccccc}
\# & {[\mathrm{~min}]} & {[\mathrm{min}]} & {\left[\mathrm{mAU}{ }^{*}\right]} & {[\mathrm{mAU}]} & \% \\
\hline 1 & 7.305 \text { BB } & 0.2193 & 1.58295 \mathrm{e} 4 & 1117.44946 & 50.4117
\end{array}
\end{aligned}
$$

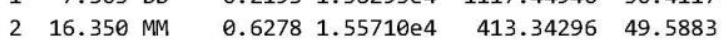


Data File D: \CHEM32\...N\C-20191108-OD-H-85-15-1ML-30MIN 2019-11-08 10-04-13\002-P2-D2-N.D Sample Name: $\mathrm{N}$

\begin{tabular}{|c|c|c|c|}
\hline Acq. Operator & : SYSTEM & Seq. Line : & 2 \\
\hline Acq. Instrument & $: 1260-D A D$ & Location : & $P 2-D-02$ \\
\hline Injection Date & : 11/8/2019 10:07:30 & $\begin{array}{r}\text { Inj : } \\
\text { Inj Volume : }\end{array}$ & $\begin{array}{cc}: & 1 \\
: & 2.000 \mu l\end{array}$ \\
\hline
\end{tabular}

Different Inj Volume from Sample Entry! Actual Inj Volume : $3.000 \mu \mathrm{l}$

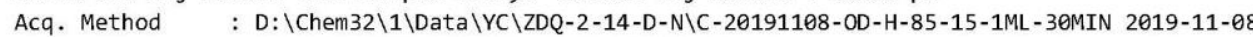
10-04-13\ZDQ-OD-H-85-15-1ML-30MIN.M

Last changed : 11/8/2019 $10: 07: 57$ by SYSTEM

(modified after loading)

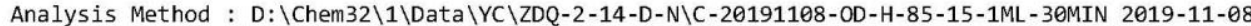
10-04-13 \ZDQ-OD-H-85-15-1ML-30MIN.M (Sequence Method)

Last changed : 11/13/2019 $20: 47: 20$ by SYSTEM

(modified after loading)

Additional Info : Peak(s) manually integrated

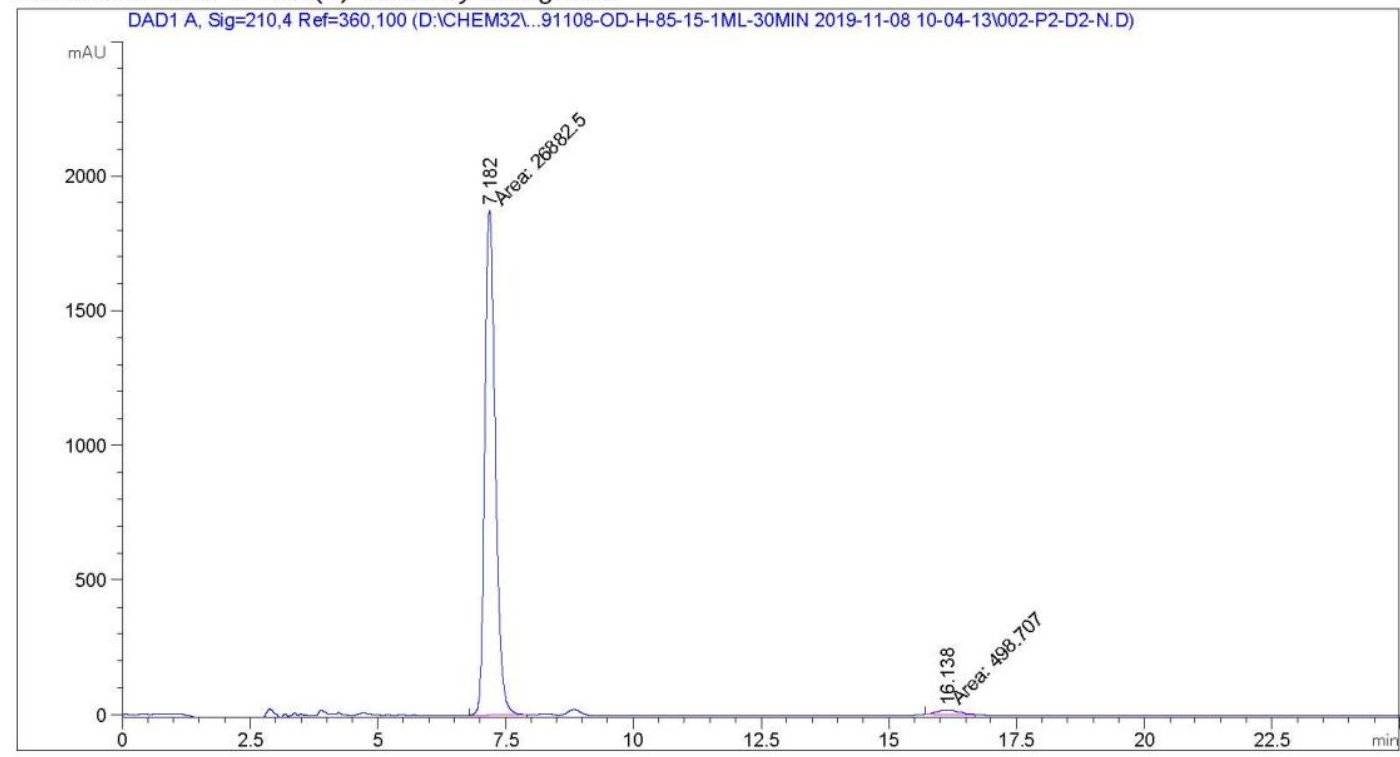

Area Percent Report

\begin{tabular}{|c|c|c|c|c|}
\hline Sorted By & : & Signal & & \\
\hline Multiplier & : & 1.0000 & & \\
\hline Dilution & : & 1.0000 & & \\
\hline \multicolumn{5}{|c|}{ Use Multiplier \& Dilution Factor with ISTDs } \\
\hline \multicolumn{5}{|c|}{ Signal 1: DAD1 A, Sig=210,4 Ref $=360,100$} \\
\hline $\begin{array}{l}\text { Peak RetTime Type } \\
\# \quad[\text { min] }\end{array}$ & $\begin{array}{l}\text { Width } \\
\text { [min] }\end{array}$ & $\begin{array}{c}\text { Area } \\
{[\mathrm{mAU} * \mathrm{~s}]}\end{array}$ & $\begin{array}{l}\text { Height } \\
{[\mathrm{mAU}]}\end{array}$ & $\begin{array}{c}\text { Area } \\
\%\end{array}$ \\
\hline 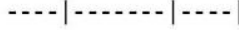 & 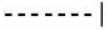 & | & $|-\cdots-\cdots|$ & - \\
\hline $17.182 \mathrm{MM}$ & 0.2389 & $2.68825 e 4$ & 1875.47766 & 98.1787 \\
\hline 216.138 MM & 0.5302 & 498.70651 & 15.67632 & 1.8213 \\
\hline
\end{tabular}




\section{References}

1. E. Peter, K.; Cunningham, A. 1,3-Benzodithiole tetraoxide as a $\mathrm{CH}_{2}{ }^{2-}$ synthon. Tetrahedron, 1988, 44, 68556860.

2. Wang, Y.; Huang, X. Disulfonyl methane as an umplung reagent: a convenient synthesis of aldehydes. Chin. J. Org. Chem., 1993, 13, 253-255.

3. Ohmiya, H.; Tsuji, T.; Yorimitsu, H. Cobalt-Catalyzed Cross-Coupling Reactions of Alkyl Halides with Allylic and Benzylic Grignard Reagents and Their Application to Tandem Radical Cyclization/Cross-Coupling Reactions. Chem. Eur. J., 2004, 10, 5640-5648.

4. T. Doi, Y. Iijima, M. Takasaki, T. Takahashi, The Journal of Organic Chemistry 2007, 72, 3667-3671. 